\title{
Extensive microbial diversity within the chicken gut microbiome revealed by metagenomics and culture
}

\author{
Rachel Gilroy ${ }^{1}$, Anuradha Ravi ${ }^{1}$, Maria Getino ${ }^{2}$, Isabella Pursley ${ }^{2}$, Daniel L. Horton ${ }^{2}$, Nabil-Fareed Alikhan ${ }^{1}$, \\ Dave Baker ${ }^{1}$, Karim Gharbi ${ }^{3}$, Neil Hall ${ }^{3,4}$, Mick Watson ${ }^{5}$, Evelien M. Adriaenssens ${ }^{1}$, Ebenezer Foster-Nyarko ${ }^{1}$, \\ Sheikh Jarju ${ }^{6}$, Arss Secka ${ }^{7}$, Martin Antonio ${ }^{6}$, Aharon Oren ${ }^{8}$, Roy R. Chaudhuri ${ }^{9}$, Roberto La Ragione ${ }^{2}$, Falk \\ Hildebrand ${ }^{\text {Corresp., 1, } 3 \text {, Mark J Pallen }}{ }^{\text {Corresp. 1, 2, } 4}$ \\ ${ }^{1}$ Quadram Institute Bioscience, Norwich, United Kingdom \\ 2 School of Veterinary Medicine, University of Surrey, Guildford, United Kingdom \\ 3 Earlham Institute, Norwich Research Park, Norwich, United Kingdom \\ 4 University of East Anglia, Norwich, United Kingdom \\ 5 Roslin Institute, University of Edinburgh, Edinburgh, United Kingdom \\ 6 Medical Research Council Unit The Gambia at the London School of Hygiene and Tropical Medicine, Atlantic Boulevard, Banjul, The Gambia \\ 7 West Africa Livestock Innovation Centre, Banjul, The Gambia \\ 8 Department of Plant and Environmental Sciences, The Alexander Silberman Institute of Life Sciences, Edmond J. Safra Campus, Hebrew University of \\ Jerusalem, Jerusalem, Israel \\ 9 Department of Molecular Biology and Biotechnology, University of Sheffield, Sheffield, United Kingdom \\ Corresponding Authors: Falk Hildebrand, Mark J Pallen \\ Email address: Falk.Hildebrand@quadram.ac.uk, mark.pallen@quadram.ac.uk
}

Background. The chicken is the most abundant food animal in the world. However, despite its importance, the chicken gut microbiome remains largely undefined. Here, we exploit culture-independent and culture-dependent approaches to reveal extensive taxonomic diversity within complex microbial community.

Results. We performed metagenomic sequencing of fifty chicken faecal samples from two breeds and analysed these, alongside all $(n=582)$ relevant publicly available chicken metagenomes, to cluster over 20 million non-redundant genes and to construct over 5,500 metagenome-assembled bacterial genomes. In addition, we recovered nearly 600 bacteriophage genomes. This represents the most comprehensive view of taxonomic diversity within the chicken gut microbiome to date, encompassing hundreds of novel candidate bacterial genera and species. To provide a stable, clear and memorable nomenclature for novel species, we devised a scalable combinatorial system for the creation of hundreds of well-formed Latin binomials. We cultured and genome-sequenced bacterial isolates from chicken faeces, documenting over forty novel species, together with three species from the genus Escherichia, including the newly named species Escherichia whittamii.

Conclusions. Our metagenomic and culture-based analyses provide new insights into the bacterial, archaeal and bacteriophage components of the chicken gut microbiome. The resulting datasets expand the known diversity of the chicken gut microbiome and provide a key resource for future high-resolution taxonomic and functional studies on the chicken gut microbiome. 
1 Extensive microbial diversity within the chicken gut microbiome revealed by metagenomics 2 and culture

3

4

5

6

7

8

Rachel Gilroy ${ }^{1}$, Anuradha Ravi1, , Maria Getino², Isabella Pursley², Daniel L. Horton², NabilFareed Alikhan ${ }^{1}$, Dave Baker ${ }^{1}$, Karim Gharbi ${ }^{3}$, Neil Hall ${ }^{3,4}$, Michael Watson ${ }^{5}$, Evelien M. Adriaenssens ${ }^{1}$, Ebenezer Foster-Nyarko ${ }^{1}$, Sheikh Jarju ${ }^{6}$, Arss Secka ${ }^{7}$, Martin Antonio ${ }^{6}$, Aharon Oren $^{8}$, Roy Chaudhuri ${ }^{9}$, Roberto M. La Ragione ${ }^{2}$, Falk Hildebrand*1,3, Mark J. Pallen ${ }^{1,2,4 *}$

${ }^{1}$ Quadram Institute Bioscience, Norwich, United Kingdom

${ }^{2}$ School of Veterinary Medicine, University of Surrey, Guildford, United Kingdom

${ }^{3}$ Earlham Institute, Norwich Research Park, Norwich, United Kingdom

${ }^{4}$ University of East Anglia, Norwich, United Kingdom

${ }^{5}$ Roslin Institute, University of Edinburgh, Edinburgh, United Kingdom

${ }^{6}$ Medical Research Council Unit The Gambia at the London School of Hygiene and Tropical Medicine, Atlantic Boulevard, Fajara, The Gambia

${ }^{7}$ West Africa Livestock Innovation Centre, Banjul, The Gambia

${ }^{8}$ Department of Plant and Environmental Sciences, The Alexander Silberman Institute of Life

Sciences, Edmond J. Safra Campus, Hebrew University of Jerusalem, Jerusalem, Israel

${ }^{9}$ Department of Molecular Biology and Biotechnology, University of Sheffield, Sheffield, United Kingdom

"Current address: Gemini centre for Sepsis research, Norwegian University of Science and Technology, Trondheim, Norway

*correspondence: mark.pallen@quadram.ac.uk; falk.hildebrand@quadram.ac.uk

\begin{abstract}
Background. The chicken is the most abundant food animal in the world. However, despite its importance, the chicken gut microbiome remains largely undefined. Here, we exploit culture-independent and culture-dependent approaches to reveal extensive taxonomic diversity within complex microbial community.

Results. We performed metagenomic sequencing of fifty chicken faecal samples from two breeds and analysed these, alongside all $(n=582)$ relevant publicly available chicken metagenomes, to cluster over 20 million non-redundant genes and to construct over 5,500 metagenome-assembled bacterial genomes. In addition, we recovered nearly 600 bacteriophage genomes. This represents the most comprehensive view of taxonomic diversity within the chicken gut microbiome to date, encompassing hundreds of novel candidate bacterial genera and species. To provide a stable, clear and memorable nomenclature for novel species, we devised a scalable combinatorial system for the creation of hundreds of well-formed Latin binomials. We cultured and genome-sequenced bacterial isolates from chicken faeces, documenting over forty novel species, together with three species from the genus Escherichia, including the newly named species Escherichia whittamii.

Conclusions. Our metagenomic and culture-based analyses provide new insights into the bacterial, archaeal and bacteriophage components of the chicken gut microbiome. The resulting datasets expand the known diversity of the chicken gut microbiome and provide a key resource for future high-resolution taxonomic and functional studies on the chicken gut microbiome.
\end{abstract}




\section{Introduction}

48 The domestic chicken is the most abundant bird and most abundant food animal on Earth,

49

50

51

52

53

54

55

56

57

58

59

60

61

62

63

64

65

66

67

68

69

70

71

72

73

74

75

76

77

78

79

80

81

82

83

84

85

86

87

88

89

90

91

92 accounting for a larger fraction of the planet's biomass than all species of wild birds combined (Bennett et al., 2018). Consumption of chicken meat is growing faster than any other type of meat and is seen as a cheaper, healthier, low-carbon alternative to meat from mammalian livestock (Eshel et al., 2014; Willett et al., 2019). Chicken eggs remain a nutritious, affordable food across the globe (Réhault-Godbert et al., 2019).

The chicken gastrointestinal tract is home to a complex community of microbes and their genes - the chicken gut microbiome - that underpins links between diet, health and productivity in poultry, as evidenced by the ability of antibiotics to promote growth in chicks (Bedford, 2000). This microbial community also acts as a source of pathogens associated with disease in birds or in humans - including Campylobacter, Salmonella, and Escherichia coli — as well as providing a reservoir of antimicrobial resistance genes (Florez-Cuadrado et al., 2018; Jørgensen et al., 2019; Hermans et al., 2012).

Previous studies of this community have documented a rich variety of microorganisms (dominated by bacteria, but including viruses, archaea and microbial eukaryotes) and have shown that the taxonomic composition of this community varies with age, breed and disease status (Shang et al., 2018; Rychlik, 2020). However, these earlier efforts have largely relied on analyses of molecular barcodes (in particular short 16S rRNA gene sequences), which fail to provide species-level resolution, are unable to detect viruses and reveal nothing about the genome sequences, population structures or functional repertoires of microbial species (Hillmann et al., 2018).

Two strategies have proven productive for exploring taxonomic and functional diversity in complex microbial communities (Almeida et al., 2019; Forster et al., 2019). Culture-independent approaches rely on shotgun metagenomic sequencing of DNA extracted from relevant samples, followed by bioinformatics-based community profiling and analysis (Glendinning et al., 2020; Sergeant et al., 2014). Culture-dependent approaches combine large-scale isolation of microorganisms in pure culture with whole-genome sequencing and phylogenomic analysis (Medvecky et al., 2018). To explore taxonomic novelty in the chicken gut microbiome, we generated phylogenetic profiles to document known and unknown diversity and then exploited culture-dependent and culture-independent approaches to create an unprecedented high-quality reference collection of microbial genes and genomes from the chicken gut, revealing and naming hundreds of new candidate species from this commonplace but important ecological setting.

\section{Materials \& Methods}

\section{Sample collection and storage}

Faecal samples were collected in South-East England from adult Lohmann Brown laying hens and adult Silkie hens in 2018. Birds were housed in a large outdoor run with a substrate of stone chippings and small turf enrichment beds during the day and kept in a coop overnight. They were fed a commercial layer feed, Farmgate Layer pellets and mash (ForFarmers UK Limited, Rougham, Bury St Edmunds), according to the manufacturer's instructions and no antibiotics were used. Faecal sampling was approved by the University of Surrey's NASPA ethics committee.

Sixty faecal samples were collected from the Lohmann Brown laying hens and thirty samples from the Silkie hens (six and three samples per day, respectively, for ten days). Freshly evacuated faeces from individual birds were collected in sterile containers and immediately 
93 stored at $-20{ }^{\circ} \mathrm{C}$. Samples were then transferred to the laboratory for culture or DNA extraction.

94 DNA was extracted using DNeasy PowerSoil kit (Qiagen), following manufacturer's instructions 95 and then stored at $-20^{\circ} \mathrm{C}$.

96

97

98

99

100

101

102

103

104

105

106

107

108

109

110

111

112

113

114

115

116

117

118

119

120

121

122

123

124

125

126

127

128

129

130

131

132

133

134

135

136

137

138

\section{Sequencing and subsequent workflow}

Workflow from this point forward is summarised in Fig. 1.The fifty samples yielding $>20 \mathrm{ng}$ DNA were processed according to the Low Input, Transpose Enabled (LITE) library construction pipeline (Perez-Sepulveda et al., 2020) before being subjected to paired-end (2x150bp) metagenomic sequencing on the Illumina Novaseq 6000 platform. Bioinformatics analyses were performed on the Earlham Institute's High Performance Computing cluster and on the Cloud Infrastructure for Microbial Bioinformatics (Connor et al., 2016). Sequences were assessed for quality using FastQC Version 0.11 .8 and trimmed using Trimmomatic Version 0.36, configured to a minimum read length of 40, "leading" and "trailing" settings of 3 (SLIDINGWINDOW:4:20) (Andrews, ; Bolger et al., 2014). Metagenomic sequences for all samples have been uploaded to the Sequence Read Archive under Bioproject ID PRJNA543206.

\section{Reference-based metagenomic analysis}

An initial analysis of our chicken faecal sequences using the Kraken 2 taxonomic classifier (Wood et al., 2019) was performed on custom databases representing the domestic chicken genome (GenBank assembly accession GCF_000002315.6) and the food plants Triticum aestivum (wheat), Aegilops tauschii (diploid progenitor of the D genome of hexaploid wheat) and Glycine max (soy bean): GenBank assembly accessions GCF_001957025.1, GCA_900519105.1, GCA_000004515.4. Kraken 2 revealed that $8 \%( \pm 16 \%)$ of reads originated from the chicken and at least $19 \%( \pm 21 \%)$ originated from the diet. These sequences were filtered from our dataset and excluded from subsequent analyses by keeping only reads 'Unclassified' by Kraken 2 after comparison with each database in turn.

The remaining dataset underwent taxonomic profiling using Kraken 2 against a microbial database built from all complete/representative archaeal, bacterial, fungal, protozoan, viral and UniVec_Core sequences in RefSeq (O'Leary et al., 2016) in January 2020. Bracken (Lu et al., 2017) was used to estimate taxon abundance from the Kraken 2 profiles, accepting only those taxa with $\geq 1000$ assigned reads. Bracken-database files were generated using "bracken-build" on our microbial database and visualised using KronaTools (Ondov et al., 2011).

\section{Metagenomic assembly}

We searched the NCBI BioProjects database (https://www.ncbi.nlm.nih.gov/bioproject/) in November 2019 with the term "chicken gut microbiome" and then selected nine publicly available projects that contained at least one metagenomic sequence dataset >1GByte in size: PRJEB33338, PRJNA193217, PRJNA291299, PRJNA375762, PRJNA415593, PRJNA417359, PRJEB22062, PRJNA543206, PRJNA417359, PRJNA385038, PRJNA616250. Only four of these studies were linked to research publications at the time of publication (Glendinning et al., 2020; Sergeant et al., 2014; Foster-Nyarko et al., 2020; Luiken et al., 2020)

All shotgun metagenomic reads were quality-filtered by removing reads shorter than $70 \%$ of the maximum expected read length (100 bp, $250 \mathrm{bp}$ for MiSeq data), an estimated accumulated error $>2.5$ with a probability of $\geq 0.01$ (Puente-Sánchez et al., 2016) or with an observed accumulated error $>2$, or $>1$ ambiguous position to assist assembly. If base quality dropped below 20 in a window of 15 bases at the 3 ' end, or if the accumulated error exceeded 2, reads 
139

140

141

142

143

144

145

146

147

148

149

150

151

152

153

154

155

156

157

158

159

160

161

162

163

164

165

166

167

168

169

170

171

172

173

174

175

176

177

178

179

180

181

182

183

184

were trimmed. All these filter steps are integrated in sdm (Hildebrand et al., 2014). Reads mapping to the chicken genome and diet were removed from the metagenomic data as described previously, classifying reads with Kraken 2 against custom databases built on the aforementioned genomes.

Sequence datasets from our fifty samples - together with 582 samples from the selected BioProjects - were assembled using MegaHIT (Li et al., 2016) under the option "--k-list $25,43,67,87,101,127$ '. To avoid artefacts that sometimes result from co-assembly of sequences from different samples and different sources, we performed individual assemblies on each sample, with the exception of BioProject PRJNA17359. For that BioProject, as multiple metagenomic samples had been sourced from different tissues of the same individual bird, we co-assembled reads from the 120 BioSamples from that project.

\section{Bacteriophage identification and characterisation}

Scaffold sequences from the MegaHIT assemblies of our fifty samples that were $\geq 10 \mathrm{~kb}$ were analysed with VirSorter v1.0.5 with the "-db 2" option to identify viral genomes (Roux et al., 2015). VirSorter Category 1 and 2 scaffold sequences were collapsed at 95\% nucleotide identity over $70 \%$ of the sequence length using CD-Hit Est v4.6.1 (Fu et al., 2012). Classification of bacteriophage sequences relied on nucleotide searches using BLASTN against the NCBI NT database (Completed April 2020) and protein searches using Kaiju Version 1.7.3 against the RefSeq database (Completed April 2020) (Menzel et al., 2016). Only bacteriophage genomes with BLASTN hit E-Value $<0.05$, percentage identity $>70 \%$ and query covering $>50 \%$ were selected as reliable hits.

A taxonomic assignment was drawn from the highest scoring BLASTN (or in rare cases BLASTP) hit ranked by query cover and percentage ID. Synteny between predicted coliphages and their respective reference genomes were visualised using EasyFig (Sullivan et al., 2011). Escherichia bacteriophage coverage per sample was determined using Anvi'o v6.1 (Eren et al., 2015) using default parameters and visualised in R using the Pheatmap package (https://www.rdocumentation.org/packages/pheatmap). Remaining viral genomes were filtered for completeness, retaining those that were circular and encoded a complete terminase gene (as predicted by VirSorter). Taxonomic assignments to family were performed on viral genomes using Demovir (https://github.com/feargalr/Demovir).

\section{Gene catalogue}

Complete genes identified by Prodigal v2.6.1 (Hyatt et al., 2010) were clustered at 95\% nucleotide identity using CD-HIT-Est v4.6.1 (Fu et al., 2012). Incomplete genes were then mapped to this complete gene list using Bowtie2 v 2.3.4.1 (Langmead \& Salzberg, 2012) and any mapping at $95 \%$ nucleotide identity were incorporated into the relevant gene clusters. Finally, genes representing the forty conserved marker genes defined by Mende et al (Mende et al., 2013) were clustered separately and then merged with the existing set of gene clusters. We thus obtained a gene catalogue of $>20$ million genes, defined as non-redundant at $95 \%$ average nucleotide identity. The final gene catalogue was uploaded to FigShare (https://doi.org/10.6084/m9.figshare.13116809.v1)

\section{Abundance estimates of contigs and genes}

Prodigal (Hyatt et al., 2010) was applied in metagenome-mode to all contigs from the MegaHIT assemblies. Unfiltered reads from each sample were mapped against their respective assembly to 
185

186

187

188

189

190

191

192

193

194

195

196

197

198

199

200

201

202

203

204

205

206

207

208

209

210

211

212

213

214

215

216

217

218

219

220

221

222

223

224

225

226

227

228

229

provide an estimate of contig and gene abundance using Bowtie2 (Langmead \& Salzberg, 2012) with the options "--no-unal--end-to-end -score-min L, -0.6,-0.6". Samtools 1.3.1 was used to sort and index all resulting Bam files (Li et al., 2009). Only reads with mapping quality $>20,>95 \%$ nucleotide identity and $>75 \%$ overall alignment length were retained. BEDTools v2.21.0 (Quinlan, 2014) was used to create depth profiles from the Bam files. These depth profiles were then translated with rdCover (https://github.com/hildebra/rdCover) into average coverage (in a $50 \mathrm{bp}$ window) per contig or per gene predicted from each contig. Bam files were translated to abundances using the "jgi_summarize_bam_contig_depths" script from the MetaBAT 2 package (Kang et al., 2019).

Gene abundances were linked to their respective gene clusters and originating samples. Redundant genes representing the same orthologue were removed.

\section{Binning}

We identified metagenomic species (MGSs) using the combinatorial approach described by Hildebrand et al (Hildebrand et al., 2019), incorporating single-assembly binning in the creation of metagenome-assembled genomes (MAGs), gene catalogue binning in the creation of canopy clusters (Nielsen et al., 2014) and hierarchical clustering of candidate genes using the $\mathrm{R}$ function hclust, method = complete. To start with, we used MetaBAT 2 v2.15 (Kang et al., 2019) to bin contigs $\geq 400 \mathrm{bp}$. These were quality filtered using CheckM v1.0.11 (Parks et al., 2015) to obtain 5,695 bins at $>80 \%$ completeness and $<5 \%$ contamination.

Species-level clusters were formed using a combination of two distinct approaches. One approach removed redundancy between samples by pre-clustering bins if $\geq 30 \%$ of their genes overlapped with a higher-quality bin to create a set of pre-MGS bins. Lower-quality bins ( $>60 \%$ completeness and $<10 \%$ contamination) were also included in the analysis but were not used to form new species clusters. To recover prokaryotic species usually obscured using single-sample assemblies and conventional binning techniques, we refined all species bins into "hcl-clusters" using gene correlations and hierarchical clustering, as described by Hildebrand et al (Hildebrand et al., 2019). We chose genes occurring in $\geq 10 \%$ of all associated MAGs as representatives for each pre-MGS bin and used these to fish for additional co-occurring genes from the gene catalogue, using a threshold of $>0.75$ Pearson correlation and $>0.85$ spearman rho to identify gene co-occurrences within this core gene set. We then merged MetaBAT 2 bins, canopy bins and co-occurring genes into our species bins. We used the presence of 40 known single-copy marker genes, without duplicates, as a quality criterion in selection of sub-clusters, before extracting the final set of MGS gene representatives using MATAFILER (https://github.com/hildebra/MATAFILER). The final collection of MGS bins (canopy clusters + hcl-clusters) was re-assessed for contamination and completeness using CheckM (Parks et al., 2015), so that we could be confident that each bin represents a single species.

A second approach de-replicated all MAGs at $95 \%$ average nucleotide identity (ANI) (species-level) and 99\% ANI (strain-level) using dRep Version 2.0 (Olm et al., 2017) and only species not identified in approach one were added to the resulting non-redundant species catalogue. The minimum aligned fraction used during ANI genome alignment was $60 \%$. A single representative MAG for each novel species cluster was uploaded to NCBI SRA under BioProject PRJNA543206 and all MAGs generated were uploaded to FigShare (https://doi.org/10.6084/m9.figshare.13116809.v1.). CompareM Version0.1.1 (https://github.com/dparks1134/CompareM) was used to calculate average amino acid identity 
230

231

232

233

234

235

236

237

238

239

240

241

242

243

244

245

246

247

248

249

250

251

252

253

254

255

256

257

258

259

260

261

262

263

264

265

266

267

268

269

270

271

272

273

274

275

between novel genera. The minimum sequence coverage for reporting an AAI alignment was set to default at $70 \%$.

\section{Taxonomy of metagenomic species}

We used the Genome Taxonomy Database Toolkit (GTDB-Tk Release 95) to perform taxonomic assignments on strain-level dereplicated MAGs (Chaumeil et al., 2019). In addition, genes from each MGS were analysed through GTDB-Tk (Release 95), proGenomes resource (Mende et al., 2017) and underwent k-mer-based taxonomic profiling using Kraken 2. In assigning taxonomy, we allowed GTDB assignments to take precedence — only when no GTDB taxonomy was available would we adopt taxonomies assigned by ProGenomes and Kraken 2 and, then, only where genus and family assignments from these sources matched. When exploiting the taxonomy assigned according genes from metagenomic species, we applied a least-commonancestor approach to unplaced taxa at higher taxonomic levels. Species distribution analyses were conducted using the Vegan package in R (R-Core-Team., 2018), before visualisation using ggplot2 (Wickham, 2016) and Pheatmap R packages

(https://www.rdocumentation.org/packages/pheatmap). Pan-genome analysis was conducted using Roary v3.11.2 and visualised using the roary2svg.pl script (Page et al., 2015). Comparison of our derived metagenomes with those of Glendinning et al. (Glendinning et al., 2020) was performed at 95\% ANI using dRep and visualised using web-tool BioVenn (Hulsen et al., 2008).

\section{Bacterial culture}

To estimate species richness and diversity, the Phyloseq package of R (R-Core-Team., 2018) was applied to the output from Bracken (Lu et al., 2017) on all of our chicken faecal metagenomic datasets. The six faecal samples that showed highest species richness and taxonomic diversity were selected for culture-based studies. Frozen faecal samples were thawed, vortexed and two $0.5 \mathrm{~g}$ aliquots (once processed aerobically, the other anaerobically) from each sample were suspended in $5 \mathrm{ml}$ PBS. Each aliquot was vortexed until homogenised, before performing serial dilutions in duplicate down to $1 \times 10^{-5}$. Processing of samples for aerobic and anaerobic culture was identical, except that, for anaerobic culture, all culture media, diluent and consumables were pre-reduced to anaerobic conditions for at least 24 hours before faecal samples were processed in a Whitley A95TG workstation.

For dilutions $10^{-3}-10^{5}, 200 \mu 1$ was plated directly on to a set of three agar plates for each culture medium (Brain Heart Infusion, Colombia Blood Agar, Yeast extract, casitone and fatty acid) with or without vancomycin supplementation at a concentration of $6 \mu \mathrm{g} / \mathrm{ml}$ (Table S1). Cultures were incubated at $37^{\circ} \mathrm{C}$ for 72 hours in their respective conditions before assessment of colony growth. Well-isolated colonies were picked according to colonial morphotype distinctive in colour, shape and size, before being re-streaked on to the growth medium from which they were sourced to confirm purity. Individual colonies were subsequently used to inoculate $2 \mathrm{ml}$ of broth based on the source culture medium, incubated at $37^{\circ} \mathrm{C}$ for a further 24 hours before bacterial DNA extraction. All isolates were archived at $-80^{\circ} \mathrm{C}$ in glycerol at $20 \%$ concentration.

\section{Genome sequencing and analysis}

Genomic DNA was extracted using a DNeasy UltraClean DNA isolation kit according to the manufacturer's instructions (Qiagen, Hilden, Germany). DNA was quantified using a Qubit ${ }^{\circledR}$ fluorometer (Invitrogen, CA, USA) high-sensitivity assay, before dilution to the required 
276

277

278

279

280

281

282

283

284

285

286

287

288

289

290

291

292

293

294

295

296

297

298

299

300

301

302

303

304

305

306

307

308

309

310

311

312

313

314

315

316

317

318

319

320

321

concentration in RNase-free water and purification on AMPure XP beads (Beckman Coulter). Sequencing libraries were prepared from $0.5 \mathrm{ng} / \mu 1$ of RNA free genomic DNA. A total of 282 isolates were included for genomic sequencing using the Nextera-XT DNA sample preparation kit (Illumina) and whole-genome sequencing performed using the Illumina NextSeq sequencing platform, generating paired-end reads $(2 \times 150 \mathrm{bp})$.

Paired-end reads were quality-assessed and trimmed using FastQC and Trimmomatic as described above. Trimmed reads were assembled into scaffolds using SPAdes version 3.13.1 (Bankevich et al., 2012). Scaffolds shorter than 500 bp were discarded from analysis. Genome contamination and completeness was assessed using CheckM version 1.0.13. To confirm assembly quality, only genomes conforming to all the following criteria were included in further analysis: (i) scaffold $\mathrm{N} 50$ of $>20 \mathrm{kbp}$ (ii) $90 \%$ of assembled bases at $>5 \mathrm{x}$ read coverage (iii) completeness of $>95 \%$ (iv) contamination of $<5 \%$ (v) complete $16 \mathrm{~S}$ rRNA gene sequence.

\section{Genome sequence taxonomic assignment}

Barrnap Version 0.9 (https://github.com/tseemann/barrnap) was applied to all genomes that passed the quality filters to extract full-length 16S rRNA gene sequences. These were then compared to NCBI 16S rRNA gene sequences from RefSeq genomes using the NCBI's webbased BLASTN facility (Altschul et al., 1990). 16S rRNA gene sequences that showed an identity of $<98.7 \%$ to known sequences were assigned to novel species, using the conservative approach in proposed minimal standards (Chun et al., 2018). We used ReferenceSeeker Version 1.6.2 (Schwengers et al., 2019) to determine average nucleotide identity (ANI) and conserved DNA values compared to RefSeq bacterial genomes (Completed March 2020) (O'Leary et al., 2016). Genomes that showed ANI $\leq 95 \%$ and conserved DNA $\leq 69 \%$ to the closest relative were designated novel species. The Genome Taxonomy Database Toolkit (GTDB-Tk Release 89) was used to perform taxonomic assignments on isolate genomes (Chaumeil et al., 2019). Genomes were clustered at $95 \%$ and $99 \%$ ANI before selection of a single representative isolate per species using dRep (Olm et al., 2017). Where a genome previously designated as novel clustered with a genome of assigned taxonomy, this taxonomy was then applied to the previously designated 'novel' genome. Final taxonomic assignments were based on genome-based ANI values derived from RefSeq and GTDB - with GTDB assignments taking precedence. A single representative genome for each novel or renamed species cluster was uploaded to NCBI SRA under BioProject PRJNA543206 and all genomes alongside respective 16S rRNA gene sequences generated were uploaded to FigShare (https://doi.org/10.6084/m9.figshare.13234556).

\section{Phylogenetic analysis}

For phylogenetic analysis of all MGS and genome sequenced isolates we used PhyloPhlAn v3.0.58 (Asnicar et al., 2020) with the "diversity high" and a proteome input predicted from all genome sequences using Prodigal v2.6.1 (Hyatt et al., 2010). Diamond v0.9.34 (Buchfink et al., 2015) was used to perform a search against 400 universal PhyloPhlAn markers. MAFFT v.7.271 (Katoh et al., 2002) was used to perform multiple sequence alignment before refinement with trimAl v.1.4 (Capella-Gutiérrez et al., 2009) and reconstruction into trees using FastTree v2.1 and RAxML v. 8.2.12 (Price et al., 2010; Stamatakis, 2014). All trees were visualised used the online iTOLv1.4 platform for visualisation and manual annotation (Letunic \& Bork, 2016). Trees were scrutinised to confirm that species and genera were monophyletic. Phylogeny for all cultured genomes unassigned at species level was confirmed as previously described against all available reference proteomes of that respective genus downloaded from NCBI. 
322

323

324

325

326

327

328

329

330

331

332

333

334

335

336

337

338

339

340

341

342

343

344

345

346

347

348

349

350

351

352

353

354

355

356

357

358

359

360

361

362

363

364

365

366

367
To investigate the phylogenetic placement of cultured isolates designated as Escherichia marmotae and Escherichia sp001660175 by GTDB, we constructed a core genome phylogenetic tree. The genomes from cultured isolates were compared to genomes representing the full diversity of the genus Escherichia. Three Salmonella genomes were included as an outgroup. The genome sequences were aligned using Mugsy (Angiuoli \& Salzberg, 2011), and alignment blocks conserved across all genomes were concatenated to produce a core genome alignment. A phylogenetic tree was constructed by maximum likelihood with 100 rapid bootstrap replicates, using the general time reversible model of nucleotide substitution with gamma correction for rate heterogeneity, as implemented in RAxML version 8.2.12 (Stamatakis, 2014).

\section{Results}

\section{Reference-based profiling documents novel diversity}

We collected faecal samples from fifty chickens reared in the UK belonging to two breeds: Lohman Browns $(n=30)$ and Silkies $(n=20)$. Short-read sequencing of fifty faecal samples generated a metagenomic dataset in excess of a billion paired-end reads or three hundred billion base pairs (Table S2).

We initially analysed the faecal samples using the k-mer-based program Kraken 2, followed by refined phylogenetic analysis using the allied program Bracken (Lu et al., 2017) (Table S3). Unsurprisingly, these programs assigned sequence reads from the faecal samples to all three domains of life, as well as to viruses (Table S4), although relative abundance assignments show that bacteria predominate in this environment. Sequences were assigned to a wide range of bacterial phyla, including the three expected as predominant in the vertebrate gut (Bacteroidetes, Firmicutes, Proteobacteria), but also including over twenty additional phyla. Searches of the PubMed database with each phylum name and the term "chicken" reveal that round half of these have been previously documented in the chicken gut. However, at least a dozen appear to be novel in this setting, including the Aquificae, Balneolaeota, Calditrichaeota, Chlorobi, Dictyoglomi, Fibrobacteres, Gemmatimonadetes, Ignavibacteriae, Kiritimatiellaeota, Lentisphaerae, Nitrospirae, and the Thermodesulfobacteria.

When we rank-ordered the species identified by Bracken according to maximum abundance in any one sample, we found, as expected, that species from the family Lactobacillaceae dominated among the top twenty most abundant organisms. However, we found that two species of Escherichia-Escherichia coli and Escherichia marmotae-accounted for $\geq 5 \%$ of reads in nearly half of the samples $(23 / 50)$ and in two samples, accounted for more than $50 \%$. Such monodominance of the gut microbiome by bacterial species has been described in diseased humans (Hildebrand et al., 2019; Ravi et al., 2019), but is surprising in the context of poultry reported as apparently healthy by their handlers. We also noted a high relative abundance of the recently described chicken pathogen Gallibacterium anatis (Narasinakuppe Krishnegowda et al., 2020) in most birds (with five birds showing $>5 \%$ reads assigned to this organism), despite their healthy status. Similarly, Fusobacterium mortiferum - an opportunistic pathogen of humans (Almohaya et al., 2020) - accounted for $>10 \%$ of sequences in ten birds, corroborating a recent report of high abundance of $16 \mathrm{~S}$ rRNA gene sequences from this organism obtained from the chicken caecum (Kollarcikova et al., 2019).

Bracken assigned sequences to over a hundred bacteriophage genomes, predominately phages infecting members of the Enterobacteriaceae assigned to the families Myoviridae and Podoviridae. Particularly noteworthy was the high abundance of reads in some samples from two distinct bacteriophages that prey on E. coli: phiEcoM-GJ1-a lytic bacteriophage isolated in 
368 Canada from pig sewage (Jamalludeen et al., 2008) — which accounted for $6.7 \%$ reads in a single 369 sample and phAPEC8 - a lytic bacteriophage with a large $147 \mathrm{~kb}$ genome, isolated from a

370 Belgian poultry farm - which accounted for $10 \%$ of reads in a single sample and for $>1 \%$ of 371 reads in three others (Tsonos et al., 2012).

372

Although these k-mer-based analyses can provide interesting insights into taxonomic diversity within the chicken gut, we quickly realised that they provide an incomplete and misleading picture of this important microbiome for several reasons: (1) they often report the presence of highly implausible organisms - for example, Kraken 2 reported the presence of human pathogens such as Shigella flexneri and Plasmodium falciparum that are simply not credible in this context on clinical grounds; (2) as with studies on 16S rRNA gene sequences, they fail to provide genomic data or insights into the functional diversity or population structure of the microbial species that they identify and; (3) they rely on a reference database and so can only report previously known organisms and can never uncover "unknown unknowns".

The scale of the problem of unknown diversity is clear from the observation that nearly three quarters $(73 \%)$ of sequence reads from our chicken samples cannot be confidently classified by Kraken 2 to species level and more than half of the reads (54\%) cannot be classified at all and are simply designated as "Unassigned". We therefore sought to extend our understanding of this community through two powerful reference-free approaches: assembly-based metagenome analyses and high-throughput culture.

\section{Metagenomic assembly uncovers a wealth of viral diversity}

\section{Assembly of metagenomic sequences is a reference-free approach that involves aligning and} merging short sequence reads into long contiguous sequences (contigs), which can then be ordered into larger scaffolds that include sequence gaps.

To confirm the presence of bacteriophages inferred through the reference-based analysis and to identify novel viral genomes, we assembled sequence reads from our fifty chicken faecal samples into scaffolds. Scaffold sequences $\geq 10 \mathrm{~kb}$ were analysed with VirSorter - a program designed to detect viral signals in microbial sequence data to find novel viruses (Roux et al., 2015).

VirSorter identified 184 of our chicken faecal scaffolds as Category 1 ("most confident") bacteriophage sequences and identified an additional 1,840 scaffolds as Category 2 ("likely") bacteriophage sequences. This was de-replicated to 1,455 genomes using similarity thresholds of $95 \%$ ANI over $70 \%$ of the genome (Table S5). BLASTN analysis revealed only ten of these bacteriophage genomes showed high similarity (percentage identity $>70 \%$; query covering $>$ $50 \%$ ) to known phages at the nucleotide level (Table S6). These included close relatives of the two phages (phiEcoM-GJ1 and phAPEC8) found highly abundant in the Bracken analyses (Fig. 2). Interestingly, more than one genus of coliphage (e.g. Jilinvirus, Phapecoctavirus, or Gamaleyavirus) was often detected in the same sample, along with an abundance of reads from their predicted prey (Escherichia) suggesting interesting dynamics in phage-host and phagephage interactions (Fig. 3; Table S7).

Of the remaining 1,445 unclassified bacteriophage genomes, nearly 600 encoded either an obvious terminase region or were circular and as such were suggested as being near-complete. Classification of these genomes revealed all genomes were predicted to belong to the order Caudovirales of tailed phages, with the majority belonging to the family Siphoviridae $(\mathrm{n}=429)$, but we also found representatives from the Myoviridae $(\mathrm{n}=87)$ and Podoviridae $(\mathrm{n}=27)$, plus

413 some bacteriophages unclassified at family level $(n=28)$ (Table S8). 

contigs/scaffolds on the basis of sequence composition and depth of coverage into discrete population bins representing metagenome-assembled genomes (MAGs). However, to carry out a definitive survey of bacterial and archaeal diversity in the chicken gut microbiome - in addition

420

421

422

423

424

425

426

427

428

429

430

431

432

433

434

435

436

437

438

439

440

441

442

443

444

445

446

447

448

449

450

451

452

453

454

455

456

457

458

459 to analysing the fifty faecal samples mentioned and before we started the binning - we retrieved all publicly available chicken gut metagenomic datasets, to create an expansive dataset representing $>630$ samples, drawn from ten studies and twelve countries (Belgium, China, France, Germany, Italy, Malaysia, Netherlands, Poland, Spain, The Gambia, UK, USA) (Figure S1a/S1b; Table S9).

Sequence assembly and binning on all these samples generated 5,595 MAGs that passed our quality threshold of $\geq 80 \%$ completion and $\leq 5 \%$ contamination (Figure S1c). Of these 3,131 could be considered high-quality draft genomes, with $>90 \%$ completion and $<5 \%$ contamination, as judged by recently published criteria (Table S10) (Bowers et al., 2017). Genome sizes of the MAGs ranged from $\sim 0.5$ to $6.4 \mathrm{Mbp}$, while GC content ranged from $24 \%$ to $73 \%$.

Then, we grouped the MAGs into metagenomic species (MGSs). Initially, this involved dereplicating MAGs at the widely accepted $95 \%$ average nucleotide identity (ANI) for defining bacterial and archaeal species and 99\% ANI for defining bacterial and archaeal strains (Jain et al., 2018; Luo et al., 2014). De-replication of MAGs at 95\% ANI resulted in 846 clusters representing bacterial and archaeal species, while de-replication at 99\% ANI resulted in 2182 clusters, representing strains. However, to improve recovery of MAGs, MGSs and associated gene sets, we used gene correlations to identify species-representative genes and then applied hierarchical clustering to co-occurring genes across the samples. This allowed us to identify additional genes from the core genome of a species, even when they show divergent nucleotide compositions (such as genes from genomic islands and plasmids) (Hildebrand et al., 2019). Similarly, using canopy clustering (Nielsen et al., 2014), we could identify commonly occurring species of low abundance. Using these approaches, we were able to identify an additional seven MGSs (Table S11). These MGS were prevalent at $>1 \mathrm{x}$ coverage in $53 \%$ of all analysed samples spanning at least 4 different BioProjects.

Analysis of bacterial metagenomic species, primarily using the Genome Taxonomy Database (GTDB) taxonomy (Parks et al., 2020), confirmed and extended the taxonomic novelty uncovered by reference-based community profiling (Fig. 4), recovering species spanning nineteen of the bacterial phyla defined by GTDB (Table S12). These include Cyanobacteria (12 species, 32 strains); Deferribacterota (1 species, 1 strain) Synergistota (2 species; 5 strains) and the Verrucomicrobiota (7 species; 8 strains).

Of the 853 de-replicated bacterial metagenomic species, 321 represented previously delineated species catalogued in publicly available databases (Table S13). Following direct comparison, a further 165 metagenomic species had been previously identified by Glendinning et al (Glendinning et al., 2020), with these sequences not currently available in public archives. However, only 158 of our metagenomic species possess validly published names based on Latin binomials.

We performed a search of PubMed with the species name and "chicken", leaving aside the 33 species named by Glendinning et al (Glendinning et al., 2020). This suggested that our study provides the first-evidence-in-chickens for the majority (81/125) of these species (Table S14). Examples include: Jeotgalicoccus halophilus, first isolated from the traditional fermented 
460

461

462

463

464

465

466

467

468

469

470

471

472

473

474

475

476

477

478

479

480

481

482

483

484

485

486

487

488

489

490

491

492

493

494

495

496

497

498

499

500

501

502

503

504

seafood, Jeotgal (Yoon et al., 2003) and present in 197 chicken samples; Aliicoccus persicus, first isolated from a hypersaline lake (Amoozegar et al., 2014) and present in 241 chicken samples; and Bacteroides reticulotermitis, first isolated from the gut of a termite (Sakamoto \& Ohkuma, 2013) and present in 39 chicken samples.

We found that 310 of our metagenomic species could be assigned a taxonomy only at the level of genus and so represent novel candidate species. A further 56 species could be assigned a taxonomy only at the level of family and, after AAI clustering at $60 \%$, were assigned to 36 novel candidate genera. One candidate bacterial species could be assigned a taxonomy only at the level of order (Oscillospirales) and so represent a new family.

Three MAGs were assigned to the domain Archaea. One represents the species Methanobrevibacter woesei - which is already known to inhabit the chicken gut (Saengkerdsub et al., 2007) — while the other two represent novel species within the genera Methanocorpusculum and UBA71.

\section{Linnaean binomials for hundreds of new candidate species}

Linnaeus first proposed the assignment of Latin binomials to provide a universal nomenclature for biological species (Linnaeus, 1759). The International Code of Nomenclature of Prokaryotes (ICNP) sets the rules for naming prokaryotic species (Parker et al., 2019), but currently precludes the valid publication of names of uncultivated organisms, represented by MAGs or other sequences. Furthermore, high-throughput generation of MAGs and of sequence-based taxonomies for bacteria, such as the GTDB (Parks et al., 2020) is often assumed to preclude the detailed attention usually given to one-by-one construction of Linnaean binomials. As a result, most uncultured taxa, as well as many taxa defined on sequence-based criteria, have been assigned unstable, confusing and hard to-remember alphanumerical identifiers.

To provide a stable, clear and memorable nomenclature for novel and/or previously unnamed bacterial and archaeal species from the chicken gut, we exploited the provision within the ICNP for naming uncultivated taxa via Candidatus assignments, which, although provisional, provide the scientific community with well-formed Latin binomials (Oren, 2017; Oren et al., 2020). However, this prompted us into an unprecedented effort to create hundreds of new names for the purpose of this single research study - an effort that required us to devise a scalable combinatorial system for the creation of binomials. Here, we made extensive combinatorial use of several dozen Latin and Greek roots pertaining to poultry (avi-, galli-, pulli-, alektryo, ptero, kotto-, ornitho-), intestines (intestini- entero-), faeces (faec-, kakke, merd-, kopro-, excrement-) or microbial life (-monas, -bacterium, -microbium, -coccus, -bacillus, -bium, -cola) — twinned with addition of these roots (singly or in tandem) and/or prefixes (allo, hetero, meta-, para-, crypto-) to existing genus names - to create over 150 Candidatus genus names. For genera with alphanumeric designations in GTDB Release 05-RS95 (Parks et al., 2020) known to occur also in gut microbiomes of other animals, we adopted a similar combinatorial approach, but avoided roots pertaining to poultry and stuck instead with combinations that simply meant "gut or faecal microbe", e.g Fimicola, Caccocola. An additional source of diversity stemmed from repetitive use of around forty Candidatus species epithets built from similar roots, which when combined with genus names gave us a total of over 650 distinctive binomials for new Candidatus species (Table 1, Table S15).

\section{Taxonomic diversity of cultured bacterial isolates}


505 To extend our metagenomics analyses, we applied culture-based methods to six faecal samples

506 that appeared species-rich in Kraken 2 analyses and in so doing obtained 282 isolates from

507 aerobic culture ( $\sim 80 \%$ of isolates) and anaerobic culture ( $20 \%$ of isolates) (Table S16). All

508 isolates underwent genome sequencing on the Illumina platform and phylogenetic analysis to

509 enable taxonomic assignment. The resulting chicken gut culture collection was found to contain

51056 genera, 93 species and 162 strains drawn from five phyla. These included thirty novel species,

511 with all novel species confirmed to originate from a monophyletic group through phylogenetic

512 analysis against all available reference genomes of their respective genus (Figure S2). Curiously,

513 there was no overlap between the species that we obtained and those reported by Medvecky et al

514 (Medvecky et al., 2018), suggesting that we are far from exhausting the set of species that can be

515 cultured from this habitat. As with the metagenomic species, all novel or previously unnamed

516 genera and species from cultured isolates were assigned Linnaean binomials (Table 2; Table

517 S17). Species-level ANI clustering of all MAGs and all cultured isolates according to phylum is

518 provided in Figure S4.

519 Interestingly, alongside ten cultured isolates of the well-characterised species Escherichia

520 coli, we recovered three isolates from Escherichia marmotae (a species recently described in

521 Himalayan marmots (Liu et al., 2015)). As previously reported, the E. marmotae strains cluster

522 closely with the Escherichia Clade V (Liu et al., 2019; Walk, 2015), so all members of this clade

523 should be considered members of this species (Fig. 5, Table S18). Further analysis of the GTDB

524 species designated Escherichia sp001660175

525 (https://gtdb.ecogenomic.org/searches?s=al\&q=sp001660175) confirmed that this species forms

526 a monophyletic lineage that corresponds to Clade II, among the cryptic environmental clades

527 described by Whittam and his colleagues (Walk et al., 2009), which was subsequently

528 documented in birds (Clermont et al., 2011). As Clade II is comparable in divergence to the other

529 Escherichia spp. and cryptic clades, we have therefore assigned the Linnaean binomial

530 Escherichia whittamii to designate a new species (Table 2), honouring the outstanding

531 contribution of Thomas S. Whittam to the study of Escherichia spp. (Walk \& Feng, 2011).

532 We found that only sixteen species were common to our cultured isolates and our MGS.

533 Subsequent sequence mapping allowed us to detect a further two cultured species at $\geq 1 \mathrm{x}$

534 coverage in at least one metagenomic sample (Fig. 6a; Table S19). The genomes from cultured

535 isolates were on average 20\% larger than the corresponding MAG sequences retrieved from the

536 same source sample (Table S20), which is in line with the completeness threshold of $80 \%$ we

537 adopted in quality assurance of the MAGs. However, when we performed detailed gene content

538 analyses on three abundant species in both cultured and metagenomic datasets - Lactobacillus

539 reuteri (with the synonym Limosilactobacillus reuteri), Escherichia coli (including the synonym

540 Escherichia flexneri) and Enterococcus faecium - we found that $>99 \%$ of the genes from the

541 core genomes and nearly half of the genes in the accessory genomes of cultured species were

542 represented in at least one MAG. These observations suggest that our high-quality MAGs are

543 sufficiently complete to warrant Candidatus names.

544 We analysed our chicken faecal metagenomes with a Kraken 2 database derived from

545 genomes representing our candidate metagenomic and cultured species, this yielded a

546 considerable improvement in the number of reads that can be classified through rapid

547 phylogenetic profiling (Fig. 6b).

548

549 Distribution of microbial species 
550

551

552

553

554

555

556

557

558

559

560

561

562

563

564

565

566

567

568

569

570

571

572

573

574

575

576

577

578

579

580

581

582

583

584

585

586

587

588

589

590

591

592

593

594

595

An analysis of the distribution of 820 MGSs across the entire metagenomic dataset revealed marked variation between samples, with not a single species present at $\geq 1 \mathrm{x}$ coverage in all samples and only 39 species present in $>90 \%$ of samples - although 441 species were present in $>50 \%$ of samples at $\geq 1 \mathrm{x}$ coverage (Table S21). At $\geq 1 \mathrm{x}$ coverage, co-occurrence of nearly 300 species $(\mathrm{n}=295$ ) was identified across all 10 BioProjects (Fig. 7a), with no species identified in all BioProjects at $\geq 10 \mathrm{x}$ coverage (Fig. 7b). Focusing on samples from distinct sites, we found no species present in all faecal samples at $>1 \mathrm{x}$ coverage and only two species were found in all caecal samples at $>1 \mathrm{x}$ coverage: both of them newly named in this study: Paraprevotella stercoravium and Blautia pullistercoris (the latter identified but not named by Glendinning et al. 2020 (Glendinning et al., 2020). These findings rule out the concept of a core chicken gut microbiome. Studies on the human gut microbiome provide a useful comparison in that, in a recent study, only fourteen genera were found to be shared across $95 \%$ of samples from the human gut (Falony et al., 2016).

Among the species with high coverage, frequency is clearly linked to Bioproject. Although species quantification curves showed that the number of species identified increased rapidly with the number of samples, species discovery appeared to plateau at approximately 230 species after including only 50 metagenomes (Figure S2a). Only two species appeared to be restricted (at $\geq 1 \mathrm{x}$ coverage) to just a single sample: Aliarcobacter thereius and Candidatus Avibacteroides faecavium. Correlation clustering confirmed structure in the data linked to BioProject (Figure S2b) - for example, the BioProject from the study by Glendinning et al (Glendinning et al., 2020) clearly shows enhancement of clostridial species compared to other BioProjects, which reflects the fact that samples in that study were sourced from chicks with no post-hatching contact with an adult bird. However, the BioSamples do not appear to cluster by country (Figure S2c) and show only limited clustering by sample site (Figure S2d). Unfortunately, there is insufficient metadata for other potentially important factors, such as breed, age or diet to draw conclusions on how these might influence clustering.

\section{Discussion}

Given the dominance of chickens in the planetary biomass, the chicken gut microbiome ranks as one of the most abundant microbial communities on the planet. Here, we have exploited two complementary approaches - metagenomics and culture - to create an extensive catalogue of genes, genomes and isolates from this important ecosystem. Our work illustrates the value of combining culture-dependent and culture-independent approaches in analysing microbiomes.

We have clearly demonstrated the advantages of shotgun metagenomic sequencing, when applied to the chicken gut microbiome, providing catalogues of genes and genome sequences that takes us well beyond what can be achieved using 16S ribosomal RNA gene sequences. Similarly, the current study is much wider in scope than the previous study by Glendinning and colleagues (Glendinning et al., 2020), not just including analyses of viral genomes and cultured isolates, while also incorporating MAGs built from data not just from that study but from all publicly available metagenomic datasets. Furthermore, the limited overlap between bacterial species represented among our cultured isolates and in our MGS reinforces the utility of the combined approach. Nonetheless, the substantial co-linearity between genomes obtained by the two approaches - and with those from another similar metagenomic study (Glendinning et al., 2020) - confirms the reliability of our binning approaches.

We were surprised to find such a remarkable phylogenetic diversity within this commonplace livestock ecosystem - diversity that rivals that associated with the human gut. Our work has

Peer) reviewing PDF | (2020:12:55980:1:1:NEW 13 Jan 2021) 
596

597

598

599

600

601

602

603

604

605

606

607

608

609

610

611

612

613

614

615

616

617

618

619

620

621

622

623

624

625

626

627

628

629

630

631

632

633

634

635

636

637

638

639

640

641 more than doubled the number of bacterial species known to reside in the chicken gut and has resulted in the creation of an unprecedented number of new Candidatus species. By including well-formed Latin binomials with the genomes we have uploaded into public repositories, we have ensured that the new proposed names and associated sequences will be integrated into commonly used online taxonomies and databases and will provide a stable taxonomic nomenclature for future studies. In addition, we have provided proof-of-principle for a scalable approach to Linnaean nomenclature that could be applied to species recovered from other metagenomic assembly projects.

Given that we did not recover by culture some of the organisms that appear most abundant by metagenomics, there is clearly scope for additional culture-based investigations, using a wider range of cultural conditions - perhaps drawing on the precedent of the Human Microbiome Project to create and target a list of the "most-wanted-for-culture" organisms documented by metagenomics (Fodor et al., 2012). The fact that novel metagenomic species are still being recovered from human gut datasets that include tens of thousands of metagenomes (Almeida et al., 2019) - twinned with the promise of novel long-read and proximity-capture approaches to metagenome analyses(Stewart et al., 2018)—-make it clear that our attempts here to analyse all currently available chicken gut metagenomes provide far from the last word on microbial diversity in this abundant and important ecosystem. Nonetheless, the availability of so many novel genes, genome and species represents a substantial step forward.

\section{Conclusions}

The extensive catalogue of genes, genomes and isolates we have created here substantially improves the coverage of the chicken gut microbiome in the public databases and will make it possible to profile sequences from the chicken gut much more rapidly, easily and comprehensively, providing a valuable resource that lays the ground-work for future comparative and intervention studies. This study also sets a provocative precedent - relevant not just to animal microbiomes, but to studies on all microbiomes - assigning well-formed Latin binomials to hundreds of metagenomic species in a scalable alternative to the automated use of bland, unstable, user-unfriendly alphanumerical designations. Drawing on the precedent set by the current study, we have recently extended this approach to encompass creation of more than a million new names for Bacteria and Archaea (Pallen et al., 2020). Thus, the time is now ripe to bring Linnaeus right into the heart of microbiome studies.

\section{Acknowledgements}

The authors thank the farmers for collecting the chicken faecal samples for the study.

\section{Citation markers for Table 2 to ensure all references included in bibliography (to be} removed during manuscript preparation)

(Chaumeil et al., 2019; Parks et al., 2020)

(Clermont et al., 2011; Gangiredla et al., 2018)

(Gupta et al., 2016)

(Hespell, 1977)

(Patel \& Gupta, 2020)

(Zheng et al., 2020) 


\section{References}

643 Almeida, A., Mitchell, A. L., Boland, M., Forster, S. C., Gloor, G. B., Tarkowska, A., Lawley, T.

644

645

646

647

648

649

650

651

652

653

654

655

656

657

658

659

660

661

662

663

664

665

666

667

668

669

670

671

672

673

674

675

676

677

678

679

680

681

682

683

684

685

686

687
D., \& Finn, R. D. (2019). A new genomic blueprint of the human gut microbiota. Nature, 568(7753), 499-504. https://doi.org/10.1038/s41586-019-0965-1

Almohaya, A. M., Almutairy, T. S., Alqahtani, A., Binkhamis, K., \& Almajid, F. M. (2020).

Fusobacterium bloodstream infections: A literature review and hospital-based case series.

Anaerobe, 62, 102165. https://doi.org/10.1016/j.anaerobe.2020.102165

Altschul, S. F., Gish, W., Miller, W., Myers, E. W., \& Lipman, D. J. (1990). Basic local

alignment search tool. J Mol Biol, 215(3), 403-410. https://doi.org/10.1016/S0022-

2836(05)80360-2

Amoozegar, M. A., Bagheri, M., Makhdoumi-Kakhki, A., Didari, M., Schumann, P., Nikou, M. M., Sánchez-Porro, C., \& Ventosa, A. (2014). Aliicoccus persicus gen. nov., sp. nov., a halophilic member of the Firmicutes isolated from a hypersaline lake. Int J Syst Evol Microbiol, 64(Pt 6), 1964-1969. https://doi.org/10.1099/ijs.0.058545-0

Andrews, S. FastQC: a quality control tool for high throughput sequence data. Retrieved 201807-26 from http://www.bioinformatics.babraham.ac.uk/projects/fastqc

Angiuoli, S. V., \& Salzberg, S. L. (2011). Mugsy: fast multiple alignment of closely related whole genomes. Bioinformatics, 27(3), 334-342.

https://doi.org/10.1093/bioinformatics/btq665

Asnicar, F., Thomas, A. M., Beghini, F., Mengoni, C., Manara, S., Manghi, P., Zhu, Q., Bolzan, M., Cumbo, F., May, U., Sanders, J. G., Zolfo, M., Kopylova, E., Pasolli, E., Knight, R., Mirarab, S., Huttenhower, C., \& Segata, N. (2020). Precise phylogenetic analysis of microbial isolates and genomes from metagenomes using PhyloPhlAn 3.0. Nat Commun, 11(1), 2500. https://doi.org/10.1038/s41467-020-16366-7

Bankevich, A., Nurk, S., Antipov, D., Gurevich, A. A., Dvorkin, M., Kulikov, A. S., Lesin, V. M., Nikolenko, S. I., Pham, S., Prjibelski, A. D., Pyshkin, A. V., Sirotkin, A. V., Vyahhi, N., Tesler, G., Alekseyev, M. A., \& Pevzner, P. A. (2012). SPAdes: a new genome assembly algorithm and its applications to single-cell sequencing. J Comput Biol, 19(5), 455-477. https://doi.org/10.1089/cmb.2012.0021

Bedford, M. (2000). Removal of antibiotic growth promoters from poultry diets: implications and strategies to minimise subsequent problems. World's Poultry Science Journal, 56(4), 347-365. https://doi.org/10.1079/WPS20000024

Bennett, C. E., Thomas, R., Williams, M., Zalasiewicz, J., Edgeworth, M., Miller, H., Coles, B., Foster, A., Burton, E. J., \& Marume, U. (2018). The broiler chicken as a signal of a human reconfigured biosphere. $R$ Soc Open Sci, 5(12), 180325.

https://doi.org/10.1098/rsos.180325

Bolger, A. M., Lohse, M., \& Usadel, B. (2014). Trimmomatic: a flexible trimmer for Illumina sequence data. Bioinformatics, 30(15), 2114-2120.

https://doi.org/10.1093/bioinformatics/btu170

Bowers, R. M., Kyrpides, N. C., Stepanauskas, R., Harmon-Smith, M., Doud, D., Reddy, T. B. K., Schulz, F., Jarett, J., Rivers, A. R., Eloe-Fadrosh, E. A., Tringe, S. G., Ivanova, N. N., Copeland, A., Clum, A., Becraft, E. D., Malmstrom, R. R., Birren, B., Podar, M., Bork, P., ... Woyke, T. (2017). Minimum information about a single amplified genome (MISAG) and a metagenome-assembled genome (MIMAG) of bacteria and archaea. Nat Biotechnol, 35(8), 725-731. https://doi.org/10.1038/nbt.3893

Buchfink, B., Xie, C., \& Huson, D. H. (2015). Fast and sensitive protein alignment using 
688

689

690

691

692

693

694

695

696

697

698

699

700

701

702

703

704

705

706

707

708

709

710

711

712

713

714

715

716

717

718

719

720

721

722

723

724

725

726

727

728

729

730

731

732

733

DIAMOND. Nat Methods, 12(1), 59-60. https://doi.org/10.1038/nmeth.3176

Capella-Gutiérrez, S., Silla-Martínez, J. M., \& Gabaldón, T. (2009). trimAl: a tool for automated alignment trimming in large-scale phylogenetic analyses. Bioinformatics, 25(15), 19721973. https://doi.org/10.1093/bioinformatics/btp348

Chaumeil, P. A., Mussig, A. J., Hugenholtz, P., \& Parks, D. H. (2019). GTDB-Tk: a toolkit to classify genomes with the Genome Taxonomy Database. Bioinformatics. https://doi.org/10.1093/bioinformatics/btz848

Chun, J., Oren, A., Ventosa, A., Christensen, H., Arahal, D. R., da Costa, M. S., Rooney, A. P., Yi, H., Xu, X. W., De Meyer, S., \& Trujillo, M. E. (2018). Proposed minimal standards for the use of genome data for the taxonomy of prokaryotes. Int J Syst Evol Microbiol, 68(1), 461-466. https://doi.org/10.1099/ijsem.0.002516

Clermont, O., Gordon, D. M., Brisse, S., Walk, S. T., \& Denamur, E. (2011). Characterization of the cryptic Escherichia lineages: rapid identification and prevalence. Environ Microbiol, 13(9), 2468-2477. https://doi.org/10.1111/j.1462-2920.2011.02519.x

Connor, T. R., Loman, N. J., Thompson, S., Smith, A., Southgate, J., Poplawski, R., Bull, M. J., Richardson, E., Ismail, M., Thompson, S. E., Kitchen, C., Guest, M., Bakke, M., Sheppard, S. K., \& Pallen, M. J. (2016). CLIMB (the Cloud Infrastructure for Microbial Bioinformatics): an online resource for the medical microbiology community. Microb Genom, 2(9), e000086. https://doi.org/10.1099/mgen.0.000086

Eren, A. M., Esen, Ö. C., Quince, C., Vineis, J. H., Morrison, H. G., Sogin, M. L., \& Delmont, T. O. (2015). Anvi'o: an advanced analysis and visualization platform for 'omics data. PeerJ, 3, e1319. https://doi.org/10.7717/peerj.1319

Eshel, G., Shepon, A., Makov, T., \& Milo, R. (2014). Land, irrigation water, greenhouse gas, and reactive nitrogen burdens of meat, eggs, and dairy production in the United States. Proc Natl Acad Sci U S A, 111(33), 11996-12001. https://doi.org/10.1073/pnas.1402183111

Falony, G., Joossens, M., Vieira-Silva, S., Wang, J., Darzi, Y., Faust, K., Kurilshikov, A., Bonder, M. J., Valles-Colomer, M., Vandeputte, D., Tito, R. Y., Chaffron, S., Rymenans, L., Verspecht, C., De Sutter, L., Lima-Mendez, G., D’hoe, K., Jonckheere, K., Homola, D., . . Raes, J. (2016). Population-level analysis of gut microbiome variation. Science, 352(6285), 560-564. https://doi.org/10.1126/science.aad3503

Florez-Cuadrado, D., Moreno, M. A., Ugarte-Ruíz, M., \& Domínguez, L. (2018). Antimicrobial Resistance in the Food Chain in the European Union. Adv Food Nutr Res, 86, 115-136. https://doi.org/10.1016/bs.afnr.2018.04.004

Fodor, A. A., DeSantis, T. Z., Wylie, K. M., Badger, J. H., Ye, Y., Hepburn, T., Hu, P., Sodergren, E., Liolios, K., Huot-Creasy, H., Birren, B. W., \& Earl, A. M. (2012). The "most wanted" taxa from the human microbiome for whole genome sequencing. PLoS One, 7, e41294. https://doi.org/10.1371/journal.pone.0041294

Forster, S. C., Kumar, N., Anonye, B. O., Almeida, A., Viciani, E., Stares, M. D., Dunn, M., Mkandawire, T. T., Zhu, A., Shao, Y., Pike, L. J., Louie, T., Browne, H. P., Mitchell, A. L., Neville, B. A., Finn, R. D., \& Lawley, T. D. (2019). A human gut bacterial genome and culture collection for improved metagenomic analyses. Nat Biotechnol, 37(2), 186-192. https://doi.org/10.1038/s41587-018-0009-7

Foster-Nyarko, E., Alikhan, N. F., Ravi, A., Thilliez, G., Thomson, N. M., Baker, D., Kay, G., Cramer, J. D., O'Grady, J., Antonio, M., \& Pallen, M. J. (2020). Genomic diversity of Escherichia coli isolates from non-human primates in the Gambia. Microb Genom, 6(9). https://doi.org/10.1099/mgen.0.000428

Peer) reviewing PDF | (2020:12:55980:1:1:NEW 13 Jan 2021) 
734

735

736

737

738

739

740

741

742

743

744

745

746

747

748

749

750

751

752

753

754

755

756

757

758

759

760

761

762

763

764

765

766

767

768

769

770

771

772

773

774

775

776

777

778

779

Fu, L., Niu, B., Zhu, Z., Wu, S., \& Li, W. (2012). CD-HIT: accelerated for clustering the nextgeneration sequencing data. Bioinformatics, 28(23), 3150-3152. https://doi.org/10.1093/bioinformatics/bts565

Gangiredla, J., Mammel, M. K., Barnaba, T. J., Tartera, C., Gebru, S. T., Patel, I. R., Leonard, S. R., Kotewicz, M. L., Lampel, K. A., Elkins, C. A., \& Lacher, D. W. (2018). Draft Genome Sequences of Escherichia albertii, Escherichia fergusonii, and Strains Belonging to Six Cryptic Lineages of Escherichia spp. Genome Announc, 6(18). https://doi.org/10.1128/genomeA.00271-18

Glendinning, L., Stewart, R. D., Pallen, M. J., Watson, K. A., \& Watson, M. (2020). Assembly of hundreds of novel bacterial genomes from the chicken caecum. Genome Biol, 21(1), 34. https://doi.org/10.1186/s13059-020-1947-1

Gupta, V., Haider, S., Sood, U., Gilbert, J. A., Ramjee, M., Forbes, K., Singh, Y., Lopes, B. S., \& Lal, R. (2016). Comparative genomic analysis of novel Acinetobacter symbionts: A combined systems biology and genomics approach. Sci Rep, 6, 29043. https://doi.org/10.1038/srep29043

Hermans, D., Pasmans, F., Messens, W., Martel, A., Van Immerseel, F., Rasschaert, G., Heyndrickx, M., Van Deun, K., \& Haesebrouck, F. (2012). Poultry as a host for the zoonotic pathogen Campylobacter jejuni. Vector Borne Zoonotic Dis, 12(2), 89-98. https://doi.org/10.1089/vbz.2011.0676

Hespell, R. B. (1977). Serpens flexibilis gen. nov., sp. nov., an Unusually Flexible, LactateOxidizing Bacterium. International Journal of Systematic Bacteriology, 27(4), 371-381. https://doi.org/10.1099/00207713-27-4-371

Hildebrand, F., Moitinho-Silva, L., Blasche, S., Jahn, M. T. T., Gossmann, T. I., Heuerta Cepas, J., Hercog, R., Luetge, M., Bahram, M., Pryszlak, A., Alves, R. J., Waszak, S. M., Zhu, A., Ye, L., Costea, P. I., Aalvink, S., Belzer, C., Forslund, S. K., Sunagawa, S., . . Bork, P. (2019). Antibiotics-induced monodominance of a novel gut bacterial order. Gut. https://doi.org/10.1136/gutjnl-2018-317715

Hildebrand, F., Tadeo, R., Voigt, A. Y., Bork, P., \& Raes, J. (2014). LotuS: an efficient and userfriendly OTU processing pipeline. Microbiome, 2(1), 30. https://doi.org/10.1186/20492618-2-30

Hillmann, B., Al-Ghalith, G. A., Shields-Cutler, R. R., Zhu, Q., Gohl, D. M., Beckman, K. B., Knight, R., \& Knights, D. (2018). Evaluating the information content of shallow shotgun metagenomics. mSystems, 3(6). https://doi.org/10.1128/mSystems.00069-18

Hulsen, T., de Vlieg, J., \& Alkema, W. (2008). BioVenn - a web application for the comparison and visualization of biological lists using area-proportional Venn diagrams. $B M C$

Genomics, 9, 488. https://doi.org/10.1186/1471-2164-9-488

Hyatt, D., Chen, G. L., Locascio, P. F., Land, M. L., Larimer, F. W., \& Hauser, L. J. (2010). Prodigal: prokaryotic gene recognition and translation initiation site identification. $B M C$ Bioinformatics, 11, 119. https://doi.org/10.1186/1471-2105-11-119

Jain, C., Rodriguez-R, L. M., Phillippy, A. M., Konstantinidis, K. T., \& Aluru, S. (2018). High throughput ANI analysis of $90 \mathrm{~K}$ prokaryotic genomes reveals clear species boundaries. Nat Commun, 9(1), 5114. https://doi.org/10.1038/s41467-018-07641-9

Jamalludeen, N., Kropinski, A. M., Johnson, R. P., Lingohr, E., Harel, J., \& Gyles, C. L. (2008). Complete genomic sequence of bacteriophage phiEcoM-GJ1, a novel phage that has myovirus morphology and a podovirus-like RNA polymerase. Appl Environ Microbiol, 74(2), 516-525. https://doi.org/10.1128/AEM.00990-07

Peer) reviewing PDF | (2020:12:55980:1:1:NEW 13 Jan 2021) 
780

781

782

783

784

785

786

787

788

789

790

791

792

793

794

795

796

797

798

799

800

801

802

803

804

805

806

807

808

809

810

811

812

813

814

815

816

817

818

819

820

821

822

823

824

825

Jørgensen, S. L., Stegger, M., Kudirkiene, E., Lilje, B., Poulsen, L. L., Ronco, T., Pires Dos Santos, T., Kiil, K., Bisgaard, M., Pedersen, K., Nolan, L. K., Price, L. B., Olsen, R. H., Andersen, P. S., \& Christensen, H. (2019). Diversity and Population Overlap between Avian and Human Escherichia coli Belonging to Sequence Type 95. mSphere, 4(1). https://doi.org/10.1128/mSphere.00333-18

Kang, D. D., Li, F., Kirton, E., Thomas, A., Egan, R., An, H., \& Wang, Z. (2019). MetaBAT 2: an adaptive binning algorithm for robust and efficient genome reconstruction from metagenome assemblies. PeerJ, 7, e7359. https://doi.org/10.7717/peerj.7359

Katoh, K., Misawa, K., Kuma, K., \& Miyata, T. (2002). MAFFT: a novel method for rapid multiple sequence alignment based on fast Fourier transform. Nucleic Acids Res, 30(14), 3059-3066. https://doi.org/10.1093/nar/gkf436

Kollarcikova, M., Kubasova, T., Karasova, D., Crhanova, M., Cejkova, D., Sisak, F., \& Rychlik, I. (2019). Use of 16S rRNA gene sequencing for prediction of new opportunistic pathogens in chicken ileal and cecal microbiota. Poult Sci, 98(6), 2347-2353. https://doi.org/10.3382/ps/pey594

Langmead, B., \& Salzberg, S. L. (2012). Fast gapped-read alignment with Bowtie 2. Nat Methods, 9(4), 357-359. https://doi.org/10.1038/nmeth.1923

Letunic, I., \& Bork, P. (2016). Interactive tree of life (iTOL) v3: an online tool for the display and annotation of phylogenetic and other trees. Nucleic Acids Res, 44(W1), W242-5. https://doi.org/10.1093/nar/gkw290

Li, D., Luo, R., Liu, C. M., Leung, C. M., Ting, H. F., Sadakane, K., Yamashita, H., \& Lam, T. W. (2016). MEGAHIT v1.0: A fast and scalable metagenome assembler driven by advanced methodologies and community practices. Methods, 102, 3-11. https://doi.org/10.1016/j.ymeth.2016.02.020

Li, H., Handsaker, B., Wysoker, A., Fennell, T., Ruan, J., Homer, N., Marth, G., Abecasis, G., Durbin, R., \& 1000, G. P. D. P. S. (2009). The Sequence Alignment/Map format and SAMtools. Bioinformatics, 25(16), 2078-2079. https://doi.org/10.1093/bioinformatics/btp352

Linnaeus, C. (1759). Systema Naturae (10th ed.). Laurentius Salvius.

Liu, S., Feng, J., Pu, J., Xu, X., Lu, S., Yang, J., Wang, Y., Jin, D., Du, X., Meng, X., Luo, X., Sun, H., Xiong, Y., Ye, C., Lan, R., \& Xu, J. (2019). Genomic and molecular characterisation of Escherichia marmotae from wild rodents in Qinghai-Tibet plateau as a potential pathogen. Sci Rep, 9(1), 10619. https://doi.org/10.1038/s41598-019-46831-3

Liu, S., Jin, D., Lan, R., Wang, Y., Meng, Q., Dai, H., Lu, S., Hu, S., \& Xu, J. (2015). Escherichia marmotae sp. nov., isolated from faeces of Marmota himalayana. Int J Syst Evol Microbiol, 65(7), 2130-2134. https://doi.org/10.1099/ijs.0.000228

Lu, J., Breitwieser, F. P., Thielen, P., \& Salzberg, S. L. (2017). Bracken: estimating species abundance in metagenomics data. PeerJ Computer Science, 3, e104. https://doi.org/10.7717/peerj-cs.104/supp-5

Luiken, R. E. C., Van Gompel, L., Bossers, A., Munk, P., Joosten, P., Hansen, R. B., Knudsen, B. E., García-Cobos, S., Dewulf, J., Aarestrup, F. M., Wagenaar, J. A., Smit, L. A. M., Mevius, D. J., Heederik, D. J. J., Schmitt, H., \& EFFORT-group. (2020). Farm dust resistomes and bacterial microbiomes in European poultry and pig farms. Environ Int, 143, 105971. https://doi.org/10.1016/j.envint.2020.105971

Luo, C., Rodriguez-R, L. M., \& Konstantinidis, K. T. (2014). MyTaxa: an advanced taxonomic classifier for genomic and metagenomic sequences. Nucleic Acids Res, 42(8), e73.

Peer) reviewing PDF | (2020:12:55980:1:1:NEW 13 Jan 2021) 
826

827

828

829

830

831

832

833

834

835

836

837

838

839

840

841

842

843

844

845

846

847

848

849

850

851

852

853

854

855

856

857

858

859

860

861

862

863

864

865

866

867

868

869

870

871

https://doi.org/10.1093/nar/gku169

Medvecky, M., Cejkova, D., Polansky, O., Karasova, D., Kubasova, T., Cizek, A., \& Rychlik, I. (2018). Whole genome sequencing and function prediction of 133 gut anaerobes isolated from chicken caecum in pure cultures. BMC Genomics, 19(1), 561. https://doi.org/10.1186/s12864-018-4959-4

Mende, D. R., Letunic, I., Huerta-Cepas, J., Li, S. S., Forslund, K., Sunagawa, S., \& Bork, P. (2017). proGenomes: a resource for consistent functional and taxonomic annotations of prokaryotic genomes. Nucleic Acids Res, 45(D1), D529-D534. https://doi.org/10.1093/nar/gkw989

Mende, D. R., Sunagawa, S., Zeller, G., \& Bork, P. (2013). Accurate and universal delineation of prokaryotic species. Nat Methods, 10(9), 881-884. https://doi.org/10.1038/nmeth.2575

Menzel, P., Ng, K. L., \& Krogh, A. (2016). Fast and sensitive taxonomic classification for metagenomics with Kaiju. Nat Commun, 7, 11257. https://doi.org/10.1038/ncomms11257

Narasinakuppe Krishnegowda, D., Dhama, K., Kumar Mariappan, A., Munuswamy, P., Iqbal Yatoo, M., Tiwari, R., Karthik, K., Bhatt, P., \& Reddy, M. R. (2020). Etiology, epidemiology, pathology, and advances in diagnosis, vaccine development, and treatment of infection in poultry: a review. Veterinary Quarterly, 40(1), 16-34. https://doi.org/10.1080/01652176.2020.1712495

Nielsen, H. B., Almeida, M., Juncker, A. S., Rasmussen, S., Li, J., Sunagawa, S., Plichta, D. R., Gautier, L., Pedersen, A. G., Le Chatelier, E., Pelletier, E., Bonde, I., Nielsen, T., Manichanh, C., Arumugam, M., Batto, J. M., Quintanilha Dos Santos, M. B., Blom, N., Borruel, N., .. . MetaHIT, C. (2014). Identification and assembly of genomes and genetic elements in complex metagenomic samples without using reference genomes. Nat Biotechnol, 32(8), 822-828. https://doi.org/10.1038/nbt.2939

O’Leary, N. A., Wright, M. W., Brister, J. R., Ciufo, S., Haddad, D., McVeigh, R., Rajput, B., Robbertse, B., Smith-White, B., Ako-Adjei, D., Astashyn, A., Badretdin, A., Bao, Y., Blinkova, O., Brover, V., Chetvernin, V., Choi, J., Cox, E., Ermolaeva, O., . . Pruitt, K. D. (2016). Reference sequence (RefSeq) database at NCBI: current status, taxonomic expansion, and functional annotation. Nucleic Acids Res, 44(D1), D733-45. https://doi.org/10.1093/nar/gkv1189

Olm, M. R., Brown, C. T., Brooks, B., \& Banfield, J. F. (2017). dRep: a tool for fast and accurate genomic comparisons that enables improved genome recovery from metagenomes through de-replication. ISME J, 11(12), 2864-2868. https://doi.org/10.1038/ismej.2017.126

Ondov, B. D., Bergman, N. H., \& Phillippy, A. M. (2011). Interactive metagenomic visualization in a Web browser. BMC Bioinformatics, 12, 385. https://doi.org/10.1186/1471-2105-12$\underline{385}$

Oren, A. (2017). A plea for linguistic accuracy - also for Candidatus taxa. Int J Syst Evol Microbiol, 67(4), 1085-1094. https://doi.org/10.1099/ijsem.0.001715

Oren, A., Garrity, G. M., Parker, C. T., Chuvochina, M., \& Trujillo, M. E. (2020). Lists of names of prokaryotic Candidatus taxa. Int J Syst Evol Microbiol. https://doi.org/10.1099/ijsem.0.003789

Page, A. J., Cummins, C. A., Hunt, M., Wong, V. K., Reuter, S., Holden, M. T., Fookes, M., Falush, D., Keane, J. A., \& Parkhill, J. (2015). Roary: rapid large-scale prokaryote pan genome analysis. Bioinformatics, 31(22), 3691-3693. https://doi.org/10.1093/bioinformatics/btv421

Pallen, M. J., Telatin, A., \& Oren, A. (2020). The Next Million Names for Archaea and Bacteria. 
872

873

874

875

876

877

878

879

880

881

882

883

884

885

886

887

888

889

890

891

892

893

894

895

896

897

898

899

900

901

902

903

904

905

906

907

908

909

910

911

912

913

914

915

916

917
Preprints, https://www.preprints.org/manuscript/202010.0160/.

https://www.preprints.org/manuscript/202010.0160/download/final file

Parker, C. T., Tindall, B. J., \& Garrity, G. M. (2019). International Code of Nomenclature of Prokaryotes. International Journal of Systematic and Evolutionary Microbiology, 69(1A), S1-S111. https://doi.org/10.1099/ijsem.0.000778

Parks, D. H., Chuvochina, M., Chaumeil, P. A., Rinke, C., Mussig, A. J., \& Hugenholtz, P. (2020). A complete domain-to-species taxonomy for Bacteria and Archaea. Nat Biotechnol. https://doi.org/10.1038/s41587-020-0501-8

Parks, D. H., Imelfort, M., Skennerton, C. T., Hugenholtz, P., \& Tyson, G. W. (2015). CheckM: assessing the quality of microbial genomes recovered from isolates, single cells, and metagenomes. Genome Res, 25(7), 1043-1055. https://doi.org/10.1101/gr.186072.114

Patel, S., \& Gupta, R. S. (2020). A phylogenomic and comparative genomic framework for resolving the polyphyly of the genus Bacillus: Proposal for six new genera of Bacillus species, Peribacillus gen. nov., Cytobacillus gen. nov., Mesobacillus gen. nov., Neobacillus gen. nov., Metabacillus gen. nov. and Alkalihalobacillus gen. nov. Int J Syst Evol Microbiol, 70(1), 406-438. https://doi.org/10.1099/ijsem.0.003775

Perez-Sepulveda, B. M., Heavens, D., Pulford, C. V., Predeus, A. V., Low, R., Webster, H., Schudoma, C., Rowe, W., Lipscombe, J., \& Watkins, C. (2020). An accessible, efficient and global approach for the large-scale sequencing of bacterial genomes. bioRxiv, 10.1101/2020.07.22.200840v1. $\underline{\mathrm{h}}$

Price, M. N., Dehal, P. S., \& Arkin, A. P. (2010). FastTree 2--approximately maximumlikelihood trees for large alignments. PLoS One, 5(3), e9490. https://doi.org/10.1371/journal.pone.0009490

Puente-Sánchez, F., Aguirre, J., \& Parro, V. (2016). A novel conceptual approach to readfiltering in high-throughput amplicon sequencing studies. Nucleic Acids Res, 44(4), e40. https://doi.org/10.1093/nar/gkv1113

Quinlan, A. R. (2014). BEDTools: The Swiss-Army Tool for Genome Feature Analysis. Curr Protoc Bioinformatics, 47, 11.12.1-34. https://doi.org/10.1002/0471250953.bi1112s47

R-Core-Team. (2018). R: A language and environment for statistical computing. R Foundation for Statistical Computing, Vienna, Austria. https://www.R-project.org

Ravi, A., Halstead, F. D., Bamford, A., Casey, A., Thomson, N. M., van Schaik, W., Snelson, C., Goulden, R., Foster-Nyarko, E., Savva, G. M., Whitehouse, T., Pallen, M. J., \& Oppenheim, B. A. (2019). Loss of microbial diversity and pathogen domination of the gut microbiota in critically ill patients. Microb Genom, 5(9). https://doi.org/10.1099/mgen.0.000293

Réhault-Godbert, S., Guyot, N., \& Nys, Y. (2019). The golden egg: nutritional value, bioactivities, and emerging benefits for human Health. Nutrients, 11(3). https://doi.org/10.3390/nu11030684

Roux, S., Enault, F., Hurwitz, B. L., \& Sullivan, M. B. (2015). VirSorter: mining viral signal from microbial genomic data. PeerJ, 3, e985. https://doi.org/10.7717/peerj.985

Rychlik, I. (2020). Composition and Function of Chicken Gut Microbiota. Animals (Basel), 10(1). https://doi.org/10.3390/ani10010103

Saengkerdsub, S., Anderson, R. C., Wilkinson, H. H., Kim, W. K., Nisbet, D. J., \& Ricke, S. C. (2007). Identification and quantification of methanogenic Archaea in adult chicken ceca. Appl Environ Microbiol, 73(1), 353-356. https://doi.org/10.1128/AEM.01931-06

Sakamoto, M., \& Ohkuma, M. (2013). Bacteroides reticulotermitis sp. nov., isolated from the gut 
918

919

920

921

922

923

924

925

926

927

928

929

930

931

932

933

934

935

936

937

938

939

940

941

942

943

944

945

946

947

948

949

950

951

952

953

954

955

956

957

958

959

960

961

962

963

of a subterranean termite (Reticulitermes speratus). Int J Syst Evol Microbiol, 63(Pt 2), 691-695. https://doi.org/10.1099/ijs.0.040931-0

Schwengers, O., Hain, T., Chakraborty, T., \& Goesmann, A. (2019). ReferenceSeeker: rapid determination of appropriate reference genomes. bioRxiv, 863621.

https://doi.org/10.1101/863621

Sergeant, M. J., Constantinidou, C., Cogan, T. A., Bedford, M. R., Penn, C. W., \& Pallen, M. J. (2014). Extensive microbial and functional diversity within the chicken cecal microbiome. PLoS One, 9(3), e91941. https://doi.org/10.1371/journal.pone.0091941

Shang, Y., Kumar, S., Oakley, B., \& Kim, W. K. (2018). Chicken Gut Microbiota: Importance and Detection Technology. Front Vet Sci, 5, 254. https://doi.org/10.3389/fvets.2018.00254

Stamatakis, A. (2014). RAxML version 8: a tool for phylogenetic analysis and post-analysis of large phylogenies. Bioinformatics, 30(9), 1312-1313. https://doi.org/10.1093/bioinformatics/btu033

Stewart, R. D., Auffret, M. D., Warr, A., Wiser, A. H., Press, M. O., Langford, K. W., Liachko, I., Snelling, T. J., Dewhurst, R. J., Walker, A. W., Roehe, R., \& Watson, M. (2018).

Assembly of 913 microbial genomes from metagenomic sequencing of the cow rumen. Nat Commun, 9(1), 870. https://doi.org/10.1038/s41467-018-03317-6

Sullivan, M. J., Petty, N. K., \& Beatson, S. A. (2011). Easyfig: a genome comparison visualizer. Bioinformatics, 27(7), 1009-1010. https://doi.org/10.1093/bioinformatics/btr039

Tsonos, J., Adriaenssens, E. M., Klumpp, J., Hernalsteens, J. P., Lavigne, R., \& De Greve, H. (2012). Complete genome sequence of the novel Escherichia coli phage phAPEC8. J Virol, 86(23), 13117-13118. https://doi.org/10.1128/JVI.02374-12

Walk, S. T., \& Feng, P. C. H. (2011). Population Genetics of Bacteria: A Tribute to Thomas $S$. Whittam. ASM Press.

Walk, S. T. (2015). The "Cryptic" Escherichia. EcoSal Plus, 6(2). https://doi.org/10.1128/ecosalplus.ESP-0002-2015

Walk, S. T., Alm, E. W., Gordon, D. M., Ram, J. L., Toranzos, G. A., Tiedje, J. M., \& Whittam, T. S. (2009). Cryptic lineages of the genus Escherichia. Appl Environ Microbiol, 75(20), 6534-6544. https://doi.org/10.1128/AEM.01262-09

Wickham, H. (2016). ggplot2: Elegant Graphics for Data Analysis. Springer-Verlag.

Willett, W., Rockström, J., Loken, B., Springmann, M., Lang, T., Vermeulen, S., Garnett, T., Tilman, D., DeClerck, F., Wood, A., Jonell, M., Clark, M., Gordon, L. J., Fanzo, J., Hawkes, C., Zurayk, R., Rivera, J. A., De Vries, W., Majele Sibanda, L., . . Murray, C. J. L. (2019). Food in the Anthropocene: the EAT-Lancet Commission on healthy diets from sustainable food systems. Lancet, 393(10170), 447-492. https://doi.org/10.1016/S0140$\underline{6736(18) 31788-4}$

Wood, D. E., Lu, J., \& Langmead, B. (2019). Improved metagenomic analysis with Kraken 2. Genome Biol, 20(1), 257. https://doi.org/10.1186/s13059-019-1891-0

Yoon, J. H., Lee, K. C., Weiss, N., Kang, K. H., \& Park, Y. H. (2003). Jeotgalicoccus halotolerans gen. nov., sp. nov. and Jeotgalicoccus psychrophilus sp. nov., isolated from the traditional Korean fermented seafood jeotgal. Int J Syst Evol Microbiol, 53(Pt 2), 595602. https://doi.org/10.1099/ijs.0.02132-0

Zheng, J., Wittouck, S., Salvetti, E., Franz, C. M. A. P., Harris, H. M. B., Mattarelli, P., O’Toole, P. W., Pot, B., Vandamme, P., Walter, J., Watanabe, K., Wuyts, S., Felis, G. E., Gänzle, M. G., \& Lebeer, S. (2020). A taxonomic note on the genus Lactobacillus: Description of 23 novel genera, emended description of the genus Lactobacillus Beijerinck 1901, and union 
964 of Lactobacillaceae and Leuconostocaceae. Int J Syst Evol Microbiol, 70(4), 2782-2858.

$965 \quad$ https://doi.org/10.1099/ijsem.0.004107

966

967 


\section{Table $\mathbf{1}$ (on next page)}

Protologues for new Candidatus taxa identified from metagenomic analysis of chicken gut samples 


\section{Description of Candidatus Acetatifactor stercoripullorum sp. nov.}

Candidatus Acetatifactor stercoripullorum (ster.co.ri.pul.lo'rum. L. neut. n. stercus dung; L. masc. n. pullus a young chicken; N.L. gen. n. stercoripullorum of the faceces of young chickens)

A bacterial species identified by metagenomic analyses. This species includes all bacteria with genomes that show $\geq 95 \%$ average nucleotide identity (ANI) to the type genome, which has been assigned the MAG ID CHK195-6426 and which is available via NCBI BioSample SAMN15816622. The GC content of the type genome is $48.46 \%$ and the genome length is 3.1 Mbp.

\section{Description of Candidatus Acinetobacter avistercoris sp. nov.}

Candidatus Acinetobacter avistercoris (a.vi.ster'co.ris. L. fem. n. avis bird; L. neut. n. stercus dung; N.L. gen. n. avistercoris of bird faeces)

A bacterial species identified by metagenomic analyses. This species includes all bacteria with genomes that show $\geq 95 \%$ average nucleotide identity (ANI) to the type genome, which has been assigned the MAG ID 5402 and which is available via NCBI BioSample SAMN15816735. The $\mathrm{GC}$ content of the type genome is $38.29 \%$ and the genome length is $3.9 \mathrm{Mbp}$.

\section{Description of Candidatus Acutalibacter ornithocaccae sp. nov.}

Candidatus Acutalibacter ornithocaccae (or.ni.tho.cac'cae. Gr. masc. or fem. n. ornis, ornithos bird Gr. fem. n. kakke faeces; N.L. gen. n. ornithocaccae of bird faeces)

A bacterial species identified by metagenomic analyses. This species includes all bacteria with genomes that show $\geq 95 \%$ average nucleotide identity (ANI) to the type genome, which has been assigned the MAG ID ChiBcolR8-3208 and which is available via NCBI BioSample SAMN15816822. This is a new name for the alphanumeric GTDB species sp000435395. The $\mathrm{GC}$ content of the type genome is $62.02 \%$ and the genome length is $2.1 \mathrm{Mbp}$.

\section{Description of Candidatus Acutalibacter pullicola sp. nov.}

Candidatus Acutalibacter pullicola (pul.li'co.la. L. masc. n. pullus a young chicken; L. suff. -cola inhabitant of; N.L. n. pullicola an inhabitant of young chickens)

A bacterial species identified by metagenomic analyses. This species includes all bacteria with genomes that show $\geq 95 \%$ average nucleotide identity (ANI) to the type genome, which has been assigned the MAG ID CHK185-1770 and which is available via NCBI BioSample SAMN15816590. The GC content of the type genome is $58.43 \%$ and the genome length is 2.1 Mbp.

\section{Description of Candidatus Acutalibacter pullistercoris sp. nov.}


47 Candidatus Acutalibacter pullistercoris (pul.li.ster'co.ris. L. masc. n. pullus a young chicken; L.

49

50

51

52

53

54

55

56

57

58

59

60

61

62

63

64

65

66

67

68

69

70

neut. n. stercus dung; N.L. gen. n. pullistercoris of young chicken faeces)

A bacterial species identified by metagenomic analyses. This species includes all bacteria with genomes that show $\geq 95 \%$ average nucleotide identity (ANI) to the type genome, which has been assigned the MAG ID 1282 and which is available via NCBI BioSample SAMN15816718. The $\mathrm{GC}$ content of the type genome is $63.65 \%$ and the genome length is $2.0 \mathrm{Mbp}$.

\section{Description of Candidatus Acutalibacter stercoravium sp. nov.}

Candidatus Acutalibacter stercoravium (ster.cor.a'vi.um. L. neut. n. stercus dung; L. fem. n. avis bird; N.L. gen. n. stercoravium of bird faeces)

A bacterial species identified by metagenomic analyses. This species includes all bacteria with genomes that show $\geq 95 \%$ average nucleotide identity (ANI) to the type genome, which has been assigned the MAG ID ChiBcolR1-495 and which is available via NCBI BioSample SAMN15816868. This is a new name for the alphanumeric GTDB species sp900543555. The $\mathrm{GC}$ content of the type genome is $60.31 \%$ and the genome length is $2.0 \mathrm{Mbp}$.

\section{Description of Candidatus Acutalibacter stercorigallinarum sp. nov.}

Candidatus Acutalibacter stercorigallinarum (ster.co.ri.gal.li.na'rum. L. neut. n. stercus dung; L. fem. n. gallina hen; N.L. gen. n. stercorigallinarum of hen faeces)

A bacterial species identified by metagenomic analyses. This species includes all bacteria with genomes that show $\geq 95 \%$ average nucleotide identity (ANI) to the type genome, which has been assigned the MAG ID ChiGjej2B2-2649 and which is available via NCBI BioSample SAMN15816629. The GC content of the type genome is $63.77 \%$ and the genome length is 2.1 Mbp.

\section{Description of Candidatus Agathobaculum intestinigallinarum sp. nov.} Candidatus Agathobaculum intestinigallinarum (in.tes.ti.ni.gal.li.na'rum. L. neut. n. intestinum gut; L. fem. n. gallina hen; N.L. gen. n. intestinigallinarum of the gut of the hens)

A bacterial species identified by metagenomic analyses. This species includes all bacteria with genomes that show $\geq 95 \%$ average nucleotide identity (ANI) to the type genome, which has been assigned the MAG ID ChiGjej6B6-20540 and which is available via NCBI BioSample SAMN15816816. This is a new name for the alphanumeric GTDB species sp900555465. The $\mathrm{GC}$ content of the type genome is $60.79 \%$ and the genome length is $2.0 \mathrm{Mbp}$.

\section{Description of Candidatus Agathobaculum intestinipullorum sp. nov.}

Candidatus Agathobaculum intestinipullorum (in.tes.ti.ni.pul.lo'rum. L. neut. n. intestinum gut; L. masc. n. pullus a young chicken; N.L. gen. n. intestinipullorum of the gut of young chickens) 
93 A bacterial species identified by metagenomic analyses. This species includes all bacteria with 94 genomes that show $\geq 95 \%$ average nucleotide identity (ANI) to the type genome, which has been 95 assigned the MAG ID ChiBcec16-9926 and which is available via NCBI BioSample

96 SAMN15816670. The GC content of the type genome is $57.76 \%$ and the genome length is 1.9

97 Mbp.

98

99

100

Description of Candidatus Agathobaculum merdavium sp. nov.

101 Candidatus Agathobaculum merdavium (merd.a'vi.um. L. fem. n. merda faeces; L. fem. n. avis

102 bird; N.L. gen. n. merdavium of bird faeces)

103

104

A bacterial species identified by metagenomic analyses. This species includes all bacteria with

105

106 genomes that show $\geq 95 \%$ average nucleotide identity (ANI) to the type genome, which has been assigned the MAG ID ChiBcec15-6302 and which is available via NCBI BioSample

108 SAMN15816712. The GC content of the type genome is $57.98 \%$ and the genome length is 2.0 Mbp.

109

110

111

Description of Candidatus Agathobaculum merdigallinarum sp. nov.

112

Candidatus Agathobaculum merdigallinarum (mer.di.gal.li.na'rum. L. fem. n. merda faeces; L.

113 fem. n. gallina hen; N.L. gen. n. merdigallinarum of hen faeces)

114

115

A bacterial species identified by metagenomic analyses. This species includes all bacteria with

116 genomes that show $\geq 95 \%$ average nucleotide identity (ANI) to the type genome, which has been assigned the MAG ID ChiSjej1B19-3834 and which is available via NCBI BioSample SAMN15816715. The GC content of the type genome is $57.98 \%$ and the genome length is 2.0 Mbp.

120

121

\section{Description of Candidatus Agathobaculum merdipullorum sp. nov.}

Candidatus Agathobaculum merdipullorum (mer.di.pul.lo'rum. L. fem. n. merda faeces; L. masc. n. pullus a young chicken; N.L. gen. n. merdipullorum of the faeces of young chickens)

A bacterial species identified by metagenomic analyses. This species includes all bacteria with genomes that show $\geq 95 \%$ average nucleotide identity (ANI) to the type genome, which has been assigned the MAG ID CHK149-1869 and which is available via NCBI BioSample SAMN15816722. The GC content of the type genome is $56.28 \%$ and the genome length is 1.7 Mbp.

\section{Description of Candidatus Agathobaculum pullicola sp. nov.}

Candidatus Agathobaculum pullicola (pul.li'co.la. L. masc. n. pullus a young chicken; L. suff. cola inhabitant of; N.L. n. pullicola an inhabitant of young chickens) 
139 assigned the MAG ID 2940 and which is available via NCBI BioSample SAMN15816725. The $\mathrm{GC}$ content of the type genome is $54.80 \%$ and the genome length is $2.0 \mathrm{Mbp}$.

141

142

Description of Candidatus Agathobaculum pullistercoris sp. nov.

144 Candidatus Agathobaculum pullistercoris (pul.li.ster'co.ris. L. masc. n. pullus a young chicken; L. neut. n. stercus dung; N.L. gen. n. pullistercoris of young chicken faeces)

146

A bacterial species identified by metagenomic analyses. This species includes all bacteria with genomes that show $\geq 95 \%$ average nucleotide identity (ANI) to the type genome, which has been assigned the MAG ID CHK180-9785 and which is available via NCBI BioSample SAMN15816619. The GC content of the type genome is $58.01 \%$ and the genome length is 2.3

151

152

153

Description of Candidatus Agathobaculum stercoravium sp. nov.

156 Mbp. Candidatus Agathobaculum stercoravium (ster.cor.a'vi.um. L. neut. n. stercus dung; L. fem. n. avis bird; N.L. gen. n. stercoravium of bird faeces)

A bacterial species identified by metagenomic analyses. This species includes all bacteria with genomes that show $\geq 95 \%$ average nucleotide identity (ANI) to the type genome, which has been assigned the MAG ID ChiW21-6059 and which is available via NCBI BioSample

161

162 SAMN15816625. The GC content of the type genome is $59.83 \%$ and the genome length is 2.3 Mbp.

163

164

Description of Candidatus Agrococcus pullicola sp. nov.

166 Candidatus Agrococcus pullicola (pul.li'co.la. L. masc. n. pullus a young chicken; L. suff. -cola inhabitant of; N.L. n. pullicola an inhabitant of young chickens)

A bacterial species identified by metagenomic analyses. This species includes all bacteria with genomes that show $\geq 95 \%$ average nucleotide identity (ANI) to the type genome, which has been assigned the MAG ID ChiGjej1B1-98 and which is available via NCBI BioSample SAMN15816710. The GC content of the type genome is $63.86 \%$ and the genome length is 3.0 Mbp.

175

\section{Description of Candidatus Akkermansia intestinavium sp. nov.}

Candidatus Akkermansia intestinavium (in.tes.tin.a'vi.um. L. neut. n. intestinum gut; L. fem. n. avis bird; N.L. gen. n. intestinavium of the gut of birds)

A bacterial species identified by metagenomic analyses. This species includes all bacteria with genomes that show $\geq 95 \%$ average nucleotide identity (ANI) to the type genome, which has been assigned the MAG ID ChiGjej6B6-8097 and which is available via NCBI BioSample SAMN15816856. This is a new name for the alphanumeric GTDB species sp900548895. The GC content of the type genome is $65.09 \%$ and the genome length is $2.2 \mathrm{Mbp}$. 
Description of Candidatus Akkermansia intestinigallinarum sp. nov.

188 Candidatus Akkermansia intestinigallinarum (in.tes.ti.ni.gal.li.na'rum. L. neut. n. intestinum gut;

189

190

191

L. fem. n. gallina hen; N.L. gen. n. intestinigallinarum of the gut of the hens)

192

A bacterial species identified by metagenomic analyses. This species includes all bacteria with genomes that show $\geq 95 \%$ average nucleotide identity (ANI) to the type genome, which has been

193

194

195

196

197

Description of Candidatus Alectryobacillus gen. nov.

198 Candidatus Alectryobacillus (A.lec.try.o.ba.cil'lus. Gr. neut. n. alektryon chicken; L. masc. n. bacillus a rod; N.L. masc. n. Alectryobacillus a bacillus found in poultry)

200

201

202

203

204

205

206

207

208

209

210

211

212

213

214

215

216

217

218

219

220

221

222

223

224

225

226

227

228

229

230

A bacterial genus identified by metagenomic analyses. The genus includes all bacteria with genomes that show $\geq 60 \%$ average amino acid identity (AAI) to the type genome from the type species Alectryobacillus merdavium. This genus has been assigned by GTDB-Tk v1.3.0 working on GTDB Release 05-RS95 (Chaumeil et al., 2019; Parks et al., 2020) to the order RFN20 and to the family $C A G-826$.

\section{Description of Candidatus Alectryobacillus merdavium sp. nov.}

Candidatus Alectryobacillus merdavium (merd.a'vi.um. L. fem. n. merda faeces; L. fem. n. avis bird; N.L. gen. n. merdavium of bird faeces)

A bacterial species identified by metagenomic analyses. This species includes all bacteria with genomes that show $\geq 95 \%$ average nucleotide identity (ANI) to the type genome, which has been assigned the MAG ID 13038 and which is available via NCBI BioSample SAMN15816966. The $\mathrm{GC}$ content of the type genome is $27.10 \%$ and the genome length is $1.2 \mathrm{Mbp}$.

\section{Description of Candidatus Alectryocaccobium gen. nov.}

Candidatus Alectryocaccobium (A.lec.try.o.cac.co'bi.um. Gr. neut. n. alektryon chicken; Gr.

fem. n. kakke faeces; Gr. masc. n. bios life; N.L. neut. n. Alectryocaccobium A life form found in chicken faceces)

A bacterial genus identified by metagenomic analyses. The genus includes all bacteria with genomes that show $\geq 60 \%$ average amino acid identity (AAI) to the type genome from the type species Alectryocaccobium stercorigallinarum. This genus has been assigned by GTDB-Tk v1.3.0 working on GTDB Release 05-RS95 (Chaumeil et al., 2019; Parks et al., 2020) to the order Lachnospirales and to the family Lachnospiraceae.

\section{Description of Candidatus Alectryocaccobium stercorigallinarum sp. nov.}


231 Candidatus Alectryocaccobium stercorigallinarum (ster.co.ri.gal.li.na'rum. L. neut. n. stercus

\section{Description of Candidatus Alectryocaccomicrobium gen. nov.}

Candidatus Alectryocaccomicrobium (A.lec.try.o.cac.co.mi.cro'bi.um. Gr. neut. n. alektryon chicken; Gr. fem. n. kakke faeces; N.L. neut. n. microbium a microbe; N.L. neut. n. Alectryocaccomicrobium A microbe found in chicken faceces)

A bacterial genus identified by metagenomic analyses. The genus includes all bacteria with genomes that show $\geq 60 \%$ average amino acid identity (AAI) to the type genome from the type species Alectryocaccomicrobium excrementavium. This genus has been assigned by GTDB-Tk v1.3.0 working on GTDB Release 05-RS95 (Chaumeil et al., 2019; Parks et al., 2020) to the order Christensenellales and to the family $C A G-74$.

\section{Description of Candidatus Alectryocaccomicrobium excrementavium sp. nov.} Candidatus Alectryocaccomicrobium excrementavium (ex.cre.ment.a'vi.um. L. neut. n. excrementum excrement; L. fem. n. avis bird; N.L. gen. n. excrementavium of bird excrement)

A bacterial species identified by metagenomic analyses. This species includes all bacteria with genomes that show $\geq 95 \%$ average nucleotide identity (ANI) to the type genome, which has been assigned the MAG ID 13766 and which is available via NCBI BioSample SAMN15816965. The GC content of the type genome is $59.90 \%$ and the genome length is $3.0 \mathrm{Mbp}$.

\section{Description of Candidatus Alistipes avicola sp. nov.}

Candidatus Alistipes avicola (a.vi'co.la. L. fem. n. avis bird; L. suff. -cola inhabitant of; N.L. n. avicola inhabitant of birds)

A bacterial species identified by metagenomic analyses. This species includes all bacteria with genomes that show $\geq 95 \%$ average nucleotide identity (ANI) to the type genome, which has been assigned the MAG ID CHK169-11906 and which is available via NCBI BioSample SAMN15816659. The GC content of the type genome is $53.79 \%$ and the genome length is 1.6 Mbp.

\section{Description of Candidatus Alistipes avistercoris sp. nov.}

Candidatus Alistipes avistercoris (a.vi.ster'co.ris. L. fem. n. avis bird; L. neut. n. stercus dung; N.L. gen. n. avistercoris of bird faeces) 
277

278

279

280

281

282

283

284

285

286

287

288

289

290

291

292

293

294

295

296

297

298

299

300

301

302

303

304

305

306

307

308

309

310

311

312

313

314

315

316

317

318

319

320

321

322

A bacterial species identified by metagenomic analyses. This species includes all bacteria with genomes that show $\geq 95 \%$ average nucleotide identity (ANI) to the type genome, which has been assigned the MAG ID 653 and which is available via NCBI BioSample SAMN15816855. This is a new name for the alphanumeric GTDB species sp000434235. The GC content of the type genome is $62.33 \%$ and the genome length is $2.4 \mathrm{Mbp}$.

\section{Description of Candidatus Alistipes cottocaccae sp. nov.}

Candidatus Alistipes cottocaccae (cot.to.cac'cae. Gr. masc. n. kottos chicken Gr. fem. n. kakke faeces; N.L. gen. n. cottocaccae of chicken faeces)

A bacterial species identified by metagenomic analyses. This species includes all bacteria with genomes that show $\geq 95 \%$ average nucleotide identity (ANI) to the type genome, which has been assigned the MAG ID ChiBcec16-1783 and which is available via NCBI BioSample SAMN15816853. This is a new name for the alphanumeric GTDB species sp002161445. The $\mathrm{GC}$ content of the type genome is $60.94 \%$ and the genome length is $2.4 \mathrm{Mbp}$.

\section{Description of Candidatus Alistipes excrementavium sp. nov.}

Candidatus Alistipes excrementavium (ex.cre.ment.a'vi.um. L. neut. n. excrementum excrement; L. fem. n. avis bird; N.L. gen. n. excrementavium of bird excrement)

A bacterial species identified by metagenomic analyses. This species includes all bacteria with genomes that show $\geq 95 \%$ average nucleotide identity (ANI) to the type genome, which has been assigned the MAG ID CHK15-232 and which is available via NCBI BioSample SAMN15816809. This is a new name for the alphanumeric GTDB species sp900021155. The $\mathrm{GC}$ content of the type genome is $61.18 \%$ and the genome length is $2.2 \mathrm{Mbp}$.

\section{Description of Candidatus Alistipes excrementigallinarum sp. nov.}

Candidatus Alistipes excrementigallinarum (ex.cre.men.ti.gal.li.na'rum. L. neut. n. excrementum excrement; L. fem. n. gallina hen; N.L. gen. n. excrementigallinarum of hen excrement)

A bacterial species identified by metagenomic analyses. This species includes all bacteria with genomes that show $\geq 95 \%$ average nucleotide identity (ANI) to the type genome, which has been assigned the MAG ID CHK106-249 and which is available via NCBI BioSample

SAMN15816875. The GC content of the type genome is $63.33 \%$ and the genome length is 2.3 Mbp.

\section{Description of Candidatus Alistipes excrementipullorum sp. nov.}

Candidatus Alistipes excrementipullorum (ex.cre.men.ti.pul.lo'rum. L. neut. n. excrementum excrement; L. masc. n. pullus a young chicken; N.L. gen. n. excrementipullorum of young chicken excrement) 
323 A bacterial species identified by metagenomic analyses. This species includes all bacteria with 324 genomes that show $\geq 95 \%$ average nucleotide identity (ANI) to the type genome, which has been

325

326

327

328

329

330

331

332

333

334

335

336

337

338

339

340

341

342

343

344

345

346

347

348

349

350

351

352

353

354

355

356

357

358

359

360

361

362

363

364

365

366

367 assigned the MAG ID ChiHjej8B7-9065 and which is available via NCBI BioSample SAMN15816799. This is a new name for the alphanumeric GTDB species. The GC content of the type genome is $56.25 \%$ and the genome length is $1.7 \mathrm{Mbp}$.

\section{Description of Candidatus Alistipes faecavium sp. nov.}

Candidatus Alistipes faecavium (faec.a'vi.um. L. fem. n. faex, faecis excrement; L. fem. n. avis bird; N.L. gen. n. faecavium of bird faeces)

A bacterial species identified by metagenomic analyses. This species includes all bacteria with genomes that show $\geq 95 \%$ average nucleotide identity (ANI) to the type genome, which has been assigned the MAG ID ChiGjej2B2-19477 and which is available via NCBI BioSample

SAMN15816800. The GC content of the type genome is $62.24 \%$ and the genome length is 2.3 Mbp.

\section{Description of Candidatus Alistipes faecigallinarum sp. nov.} Candidatus Alistipes faecigallinarum (fae.ci.gal.li.na'rum. L. fem. n. faex, faecis excrement; L. fem. n. gallina hen; N.L. gen. n. faecigallinarum of chicken faeces)

A bacterial species identified by metagenomic analyses. This species includes all bacteria with genomes that show $\geq 95 \%$ average nucleotide identity (ANI) to the type genome, which has been assigned the MAG ID 6451 and which is available via NCBI BioSample SAMN15816915. The $\mathrm{GC}$ content of the type genome is $61.37 \%$ and the genome length is $2.2 \mathrm{Mbp}$.

\section{Description of Candidatus Alistipes intestinigallinarum sp. nov.}

Candidatus Alistipes intestinigallinarum (in.tes.ti.ni.gal.li.na'rum. L. neut. n. intestinum gut; L. fem. n. gallina hen; N.L. gen. n. intestinigallinarum of the gut of the hens)

A bacterial species identified by metagenomic analyses. This species includes all bacteria with genomes that show $\geq 95 \%$ average nucleotide identity (ANI) to the type genome, which has been assigned the MAG ID 5134 and which is available via NCBI BioSample SAMN15816708. The $\mathrm{GC}$ content of the type genome is $59.58 \%$ and the genome length is $2.7 \mathrm{Mbp}$.

\section{Description of Candidatus Alistipes intestinipullorum sp. nov.}

Candidatus Alistipes intestinipullorum (in.tes.ti.ni.pul.lo'rum. L. neut. n. intestinum gut; L. masc. n. pullus a young chicken; N.L. gen. n. intestinipullorum of the gut of young chickens)

A bacterial species identified by metagenomic analyses. This species includes all bacteria with genomes that show $\geq 95 \%$ average nucleotide identity (ANI) to the type genome, which has been assigned the MAG ID ChiGjej2B2-5998 and which is available via NCBI BioSample 
368 SAMN15816759. The GC content of the type genome is $59.58 \%$ and the genome length is 2.3

369 Mbp.

370

371

372

Description of Candidatus Alistipes merdavium sp. nov.

373

Candidatus Alistipes merdavium (merd.a'vi.um. L. fem. n. merda faeces; L. fem. n. avis bird;

374

375

376

377

378

N.L. gen. n. merdavium of bird faeces)

A bacterial species identified by metagenomic analyses. This species includes all bacteria with genomes that show $\geq 95 \%$ average nucleotide identity (ANI) to the type genome, which has been assigned the MAG ID ChiBcolR5-1230 and which is available via NCBI BioSample

379 SAMN15816813. This is a new name for the alphanumeric GTDB species sp900544265. The

380

381

382

383

384

385

386

387 $\mathrm{GC}$ content of the type genome is $63.44 \%$ and the genome length is $2.2 \mathrm{Mbp}$.

\section{Description of Candidatus Alistipes merdigallinarum sp. nov.}

Candidatus Alistipes merdigallinarum (mer.di.gal.li.na'rum. L. fem. n. merda faeces; L. fem. n. gallina hen; N.L. gen. n. merdigallinarum of hen faeces)

A bacterial species identified by metagenomic analyses. This species includes all bacteria with genomes that show $\geq 95 \%$ average nucleotide identity (ANI) to the type genome, which has been

389 assigned the MAG ID 2432 and which is available via NCBI BioSample SAMN15816893.

390

391 Although GTDB has assigned this species to the genus it calls Alistipes_A, this genus designation cannot be incorporated into a well-formed binomial, so in naming this species, we have used the current validly published name for the genus. The GC content of the type genome is $49.96 \%$ and the genome length is $2.2 \mathrm{Mbp}$.

394

395

396

\section{Description of Candidatus Alistipes merdipullorum sp. nov.}

398 Candidatus Alistipes merdipullorum (mer.di.pul.lo'rum. L. fem. n. merda faeces; L. masc. n. pullus a young chicken; N.L. gen. n. merdipullorum of the faeces of young chickens)

399

400

401

402

403

404

405

406

407

408

409

410

A bacterial species identified by metagenomic analyses. This species includes all bacteria with genomes that show $\geq 95 \%$ average nucleotide identity (ANI) to the type genome, which has been assigned the MAG ID ChiHjej9B8-3741 and which is available via NCBI BioSample SAMN15816807. This is a new name for the alphanumeric GTDB species sp900546065. The $\mathrm{GC}$ content of the type genome is $57.66 \%$ and the genome length is $2.3 \mathrm{Mbp}$.

\section{Description of Candidatus Alistipes pullicola sp. nov.}

Candidatus Alistipes pullicola (pul.li'co.la. L. masc. n. pullus a young chicken; L. suff. -cola inhabitant of; N.L. n. pullicola an inhabitant of young chickens)

412

A bacterial species identified by metagenomic analyses. This species includes all bacteria with genomes that show $\geq 95 \%$ average nucleotide identity (ANI) to the type genome, which has been

413 assigned the MAG ID ChiHjej10B9-11434 and which is available via NCBI BioSample 
414 SAMN15816929. This is a new name for the alphanumeric GTDB species sp900546005.

415 Although GTDB has assigned this species to the genus it calls Alistipes_A, this genus

416 designation cannot be incorporated into a well-formed binomial, so in naming this species, we

417 have used the current validly published name for the genus. The GC content of the type genome

418 is $52.02 \%$ and the genome length is $1.9 \mathrm{Mbp}$.

419

420

421

422

Description of Candidatus Alistipes pullistercoris sp. nov.

Candidatus Alistipes pullistercoris (pul.li.ster'co.ris. L. masc. n. pullus a young chicken; L. neut. n. stercus dung; N.L. gen. n. pullistercoris of young chicken faeces)

424

425

A bacterial species identified by metagenomic analyses. This species includes all bacteria with

426

427 genomes that show $\geq 95 \%$ average nucleotide identity (ANI) to the type genome, which has been assigned the MAG ID 3244 and which is available via NCBI BioSample SAMN15816930. This is a new name for the alphanumeric GTDB species sp900240235. Although GTDB has assigned this species to the genus it calls Alistipes_A, this genus designation cannot be incorporated into a well-formed binomial, so in naming this species, we have used the current validly published name for the genus. The GC content of the type genome is $56.88 \%$ and the genome length is 2.0 Mbp.

433

434

435

Description of Candidatus Alistipes stercoravium sp. nov.

436

437

438

439

440

441

442

443

444

Candidatus Alistipes stercoravium (ster.cor.a'vi.um. L. neut. n. stercus dung; L. fem. n. avis bird; N.L. gen. n. stercoravium of bird faeces)

A bacterial species identified by metagenomic analyses. This species includes all bacteria with genomes that show $\geq 95 \%$ average nucleotide identity (ANI) to the type genome, which has been assigned the MAG ID ChiHjej8B7-9257 and which is available via NCBI BioSample

SAMN15816640. The GC content of the type genome is $61.39 \%$ and the genome length is 2.0 Mbp.

445

446

447

Description of Candidatus Alistipes stercorigallinarum sp. nov.

Candidatus Alistipes stercorigallinarum (ster.co.ri.gal.li.na'rum. L. neut. n. stercus dung; L. fem.

448

449

450

451

452

453

n. gallina hen; N.L. gen. n. stercorigallinarum of hen faeces)

A bacterial species identified by metagenomic analyses. This species includes all bacteria with genomes that show $\geq 95 \%$ average nucleotide identity (ANI) to the type genome, which has been assigned the MAG ID ChiHcolR4-13572 and which is available via NCBI BioSample SAMN15816817. This is a new name for the alphanumeric GTDB species sp900542505. The 454 455 456 GC content of the type genome is $62.42 \%$ and the genome length is $2.2 \mathrm{Mbp}$.

\section{Description of Candidatus Alistipes stercoripullorum sp. nov.}

Candidatus Alistipes stercoripullorum (ster.co.ri.pul.lo'rum. L. neut. n. stercus dung; L. masc. n. 459 pullus a young chicken; N.L. gen. n. stercoripullorum of the faceces of young chickens) 
460

461

462

463

464

465

466

467

468

469

470

471

472

473

474

475

476

477

478

479

480

481

482

483

484

485

486

487

488

489

490

491

492

493

494

495

496

497

498

499

500

501

502

503

504

505

A bacterial species identified by metagenomic analyses. This species includes all bacteria with genomes that show $\geq 95 \%$ average nucleotide identity (ANI) to the type genome, which has been assigned the MAG ID ChiBcec8-6454 and which is available via NCBI BioSample SAMN15816818. This is a new name for the alphanumeric GTDB species sp006542685. The $\mathrm{GC}$ content of the type genome is $62.87 \%$ and the genome length is $2.4 \mathrm{Mbp}$.

\section{Description of Candidatus Anaerobiospirillum merdipullorum sp. nov.} Candidatus Anaerobiospirillum merdipullorum (mer.di.pul.lo'rum. L. fem. n. merda faeces; L. masc. n. pullus a young chicken; N.L. gen. n. merdipullorum of the faeces of young chickens)

A bacterial species identified by metagenomic analyses. This species includes all bacteria with genomes that show $\geq 95 \%$ average nucleotide identity (ANI) to the type genome, which has been assigned the MAG ID 687 and which is available via NCBI BioSample SAMN15816911.

Although GTDB has assigned this species to the genus it calls Anaerobiospirillum_A, this genus designation cannot be incorporated into a well-formed binomial, so in naming this species, we have used the current validly published name for the genus. The GC content of the type genome is $49.84 \%$ and the genome length is $2.0 \mathrm{Mbp}$.

\section{Description of Candidatus Anaerobiospirillum pullicola sp. nov.}

Candidatus Anaerobiospirillum pullicola (pul.li'co.la. L. masc. n. pullus a young chicken; L. suff. -cola inhabitant of; N.L. n. pullicola an inhabitant of young chickens)

A bacterial species identified by metagenomic analyses. This species includes all bacteria with genomes that show $\geq 95 \%$ average nucleotide identity (ANI) to the type genome, which has been assigned the MAG ID 378 and which is available via NCBI BioSample SAMN15816727. The $\mathrm{GC}$ content of the type genome is $52.37 \%$ and the genome length is $3.9 \mathrm{Mbp}$.

\section{Description of Candidatus Anaerobiospirillum pullistercoris sp. nov.}

Candidatus Anaerobiospirillum pullistercoris (pul.li.ster'co.ris. L. masc. n. pullus a young chicken; L. neut. n. stercus dung; N.L. gen. n. pullistercoris of young chicken faeces)

A bacterial species identified by metagenomic analyses. This species includes all bacteria with genomes that show $\geq 95 \%$ average nucleotide identity (ANI) to the type genome, which has been assigned the MAG ID USASDec5-558 and which is available via NCBI BioSample SAMN15816730. The GC content of the type genome is $49.01 \%$ and the genome length is 3.3 Mbp.

\section{Description of Candidatus Anaerobiospirillum stercoravium sp. nov.}

Candidatus Anaerobiospirillum stercoravium (ster.cor.a'vi.um. L. neut. n. stercus dung; L. fem. n. avis bird; N.L. gen. n. stercoravium of bird faeces)

Peer) reviewing PDF | (2020:12:55980:1:1:NEW 13 Jan 2021) 
506 A bacterial species identified by metagenomic analyses. This species includes all bacteria with 507 genomes that show $\geq 95 \%$ average nucleotide identity (ANI) to the type genome, which has been 508 assigned the MAG ID USASDcec2-551 and which is available via NCBI BioSample

509 SAMN15816778. The GC content of the type genome is $56.27 \%$ and the genome length is 2.9

510 Mbp.

511

512

513

514

515

516

517 A bacterial species identified by metagenomic analyses. This species includes all bacteria with

518

519

520

521

522

523

524

525

526

527

528

529

530

531

532

533

534

535

536

537

538

539

540

541

542

543

544

Description of Candidatus Anaerobutyricum avicola sp. nov.

Candidatus Anaerobutyricum avicola (a.vi'co.la. L. fem. n. avis bird; L. suff. -cola inhabitant of; N.L. n. avicola inhabitant of birds) genomes that show $\geq 95 \%$ average nucleotide identity (ANI) to the type genome, which has been assigned the MAG ID ChiSxjej6B18-9268 and which is available via NCBI BioSample

SAMN15816760. The GC content of the type genome is $50.20 \%$ and the genome length is 2.5 Mbp.

\section{Description of Candidatus Anaerobutyricum faecale sp. nov.}

Candidatus Anaerobutyricum faecale (fae.ca'le. L. neut. adj. faecale of faeces)

A bacterial species identified by metagenomic analyses. This species includes all bacteria with genomes that show $\geq 95 \%$ average nucleotide identity (ANI) to the type genome, which has been assigned the MAG ID CHK182-24705 and which is available via NCBI BioSample SAMN15816814. This is a new name for the alphanumeric GTDB species sp002161065. The $\mathrm{GC}$ content of the type genome is $48.07 \%$ and the genome length is $2.8 \mathrm{Mbp}$.

\section{Description of Candidatus Anaerobutyricum stercoripullorum sp. nov.}

Candidatus Anaerobutyricum stercoripullorum (ster.co.ri.pul.lo'rum. L. neut. n. stercus dung; L. masc. n. pullus a young chicken; N.L. gen. n. stercoripullorum of the faceces of young chickens)

A bacterial species identified by metagenomic analyses. This species includes all bacteria with genomes that show $\geq 95 \%$ average nucleotide identity (ANI) to the type genome, which has been assigned the MAG ID ChiSxjej3B15-1167 and which is available via NCBI BioSample SAMN15816729. The GC content of the type genome is $52.36 \%$ and the genome length is 2.3 Mbp.

\section{Description of Candidatus Anaerobutyricum stercoris sp. nov.} Candidatus Anaerobutyricum stercoris (ster'co.ris. L. gen. n. stercoris of dung, excrement)

A bacterial species identified by metagenomic analyses. This species includes all bacteria with genomes that show $\geq 95 \%$ average nucleotide identity (ANI) to the type genome, which has been assigned the MAG ID CHK179-28034 and which is available via NCBI BioSample 
551 SAMN15816848. This is a new name for the alphanumeric GTDB species sp900016875. The

552

553

554

555

556

557

558

559

560

561

562

563

564

565

566

567

568

569

570

571

572

573

574

575

576

577

578

579

580

581

582

583

584

585

586

587

588

589

590

591

592

593

594

595

596

$\mathrm{GC}$ content of the type genome is $47.36 \%$ and the genome length is $3.0 \mathrm{Mbp}$.

\author{
Description of Candidatus Anaerofilum excrementigallinarum sp. nov. \\ Candidatus Anaerofilum excrementigallinarum (ex.cre.men.ti.gal.li.na'rum. L. neut. n. \\ excrementum excrement; L. fem. n. gallina hen; N.L. gen. n. excrementigallinarum of hen \\ excrement)
}

A bacterial species identified by metagenomic analyses. This species includes all bacteria with genomes that show $\geq 95 \%$ average nucleotide identity (ANI) to the type genome, which has been assigned the MAG ID 3951 and which is available via NCBI BioSample SAMN15816720. The $\mathrm{GC}$ content of the type genome is $61.37 \%$ and the genome length is $2.5 \mathrm{Mbp}$.

\title{
Description of Candidatus Anaerofilum faecale sp. nov. \\ Candidatus Anaerofilum faecale (fae.ca'le. L. neut. adj.faecale of faeces)
}

A bacterial species identified by metagenomic analyses. This species includes all bacteria with genomes that show $\geq 95 \%$ average nucleotide identity (ANI) to the type genome, which has been assigned the MAG ID ChiGjej6B6-374 and which is available via NCBI BioSample SAMN15816865. This is a new name for the alphanumeric GTDB species sp002160015. The $\mathrm{GC}$ content of the type genome is $63.11 \%$ and the genome length is $2.3 \mathrm{Mbp}$.

\section{Description of Candidatus Anaeromassilibacillus stercoravium sp. nov.}

Candidatus Anaeromassilibacillus stercoravium (ster.cor.a'vi.um. L. neut. n. stercus dung; L. fem. n. avis bird; N.L. gen. n. stercoravium of bird faeces)

A bacterial species identified by metagenomic analyses. This species includes all bacteria with genomes that show $\geq 95 \%$ average nucleotide identity (ANI) to the type genome, which has been assigned the MAG ID ChiSjej5B23-4625 and which is available via NCBI BioSample SAMN15816824. This is a new name for the alphanumeric GTDB species sp002159845. The $\mathrm{GC}$ content of the type genome is $54.17 \%$ and the genome length is $2.2 \mathrm{Mbp}$.

\section{Description of Candidatus Anaerostipes avicola sp. nov.}

Candidatus Anaerostipes avicola (a.vi'co.la. L. fem. n. avis bird; L. suff. -cola inhabitant of; N.L. n. avicola inhabitant of birds)

A bacterial species identified by metagenomic analyses. This species includes all bacteria with genomes that show $\geq 95 \%$ average nucleotide identity (ANI) to the type genome, which has been assigned the MAG ID CHK189-27985 and which is available via NCBI BioSample SAMN15816576. The GC content of the type genome is $43.22 \%$ and the genome length is 2.5 Mbp. 
597

598

599

600

601

602

603

604

605

606

607

608

609

610

611

612

613

614

615

616

617

618

619

620

621

622

623

624

625

626

627

628

629

630

631

632

633

634

635

636

637

638

639

640

641

642

\section{Description of Candidatus Anaerostipes avistercoris sp. nov.}

Candidatus Anaerostipes avistercoris (a.vi.ster'co.ris. L. fem. n. avis bird; L. neut. n. stercus dung; N.L. gen. n. avistercoris of bird faeces)

A bacterial species identified by metagenomic analyses. This species includes all bacteria with genomes that show $\geq 95 \%$ average nucleotide identity (ANI) to the type genome, which has been assigned the MAG ID ChiSjej3B21-8574 and which is available via NCBI BioSample SAMN15816634. The GC content of the type genome is $44.43 \%$ and the genome length is 2.6 Mbp.

\section{Description of Candidatus Anaerostipes excrementavium sp. nov.}

Candidatus Anaerostipes excrementavium (ex.cre.ment.a'vi.um. L. neut. n. excrementum excrement; L. fem. n. avis bird; N.L. gen. n. excrementavium of bird excrement)

A bacterial species identified by metagenomic analyses. This species includes all bacteria with genomes that show $\geq 95 \%$ average nucleotide identity (ANI) to the type genome, which has been assigned the MAG ID CHK191-13928 and which is available via NCBI BioSample SAMN15816615. The GC content of the type genome is $41.56 \%$ and the genome length is 2.7 Mbp.

\section{Description of Candidatus Anaerotignum merdipullorum sp. nov.}

Candidatus Anaerotignum merdipullorum (mer.di.pul.lo'rum. L. fem. n. merda faeces; L. masc. n. pullus a young chicken; N.L. gen. n. merdipullorum of the faeces of young chickens)

A bacterial species identified by metagenomic analyses. This species includes all bacteria with genomes that show $\geq 95 \%$ average nucleotide identity (ANI) to the type genome, which has been assigned the MAG ID CHK190-6203 and which is available via NCBI BioSample SAMN15816613. The GC content of the type genome is $44.75 \%$ and the genome length is 2.2 Mbp.

\section{Description of Candidatus Anaerotruncus excrementipullorum sp. nov.}

Candidatus Anaerotruncus excrementipullorum (ex.cre.men.ti.pul.lo'rum. L. neut. n. excrementum excrement; L. masc. n. pullus a young chicken; N.L. gen. n. excrementipullorum of young chicken excrement)

A bacterial species identified by metagenomic analyses. This species includes all bacteria with genomes that show $\geq 95 \%$ average nucleotide identity (ANI) to the type genome, which has been assigned the MAG ID CHK188-5543 and which is available via NCBI BioSample SAMN15816616. The GC content of the type genome is $64.05 \%$ and the genome length is 1.9 Mbp. 
643 Description of Candidatus Aphodenecus gen. nov.

644 Candidatus Aphodenecus (Aph.o.d.en.e'cus. Gr. fem. n. aphodos dung; Gr. masc. enoikos

645

646

647

648

649

650

651

652

653

654

655

656

657

658

659

660

661

662

663

664

665

inhabitant; N.L. masc. n. Aphodenecus a microbe associated with faeces)

A bacterial genus identified by metagenomic analyses. The genus includes all bacteria with genomes that show $\geq 60 \%$ average amino acid identity (AAI) to the type genome from the type species Aphodenecus pullistercoris. This is a name for the alphanumeric GTDB genus Spiro-01. This genus has been assigned by GTDB-Tk v1.3.0 working on GTDB Release 05-RS95 (Chaumeil et al., 2019; Parks et al., 2020) to the order Sphaerochaetales and to the family Sphaerochaetaceae.

\section{Description of Candidatus Aphodenecus pullistercoris sp. nov.}

Candidatus Aphodenecus pullistercoris (pul.li.ster'co.ris. L. masc. n. pullus a young chicken; L. neut. n. stercus dung; N.L. gen. n. pullistercoris of young chicken faeces)

A bacterial species identified by metagenomic analyses. This species includes all bacteria with genomes that show $\geq 95 \%$ average nucleotide identity (ANI) to the type genome, which has been assigned the MAG ID 11167 and which is available via NCBI BioSample SAMN15817123. The $\mathrm{GC}$ content of the type genome is $59.34 \%$ and the genome length is $2.0 \mathrm{Mbp}$.

\section{Description of Candidatus Aphodocola gen. nov.}

666 Candidatus Aphodocola (Aph.o.do'co.la. Gr. fem. n. aphodos dung; L. suff. -cola inhabitant of; N.L. fem. n. Aphodocola a microbe associated with faeces)

670

A bacterial genus identified by metagenomic analyses. The genus includes all bacteria with genomes that show $\geq 60 \%$ average amino acid identity (AAI) to the type genome from the type species Aphodocola excrementigallinarum. This is a name for the alphanumeric GTDB genus CAG-594. This genus has been assigned by GTDB-Tk v1.3.0 working on GTDB Release 05RS95 (Chaumeil et al., 2019; Parks et al., 2020) to the order RF39 and to the family CAG-433.

674

675

676

677

\section{Description of Candidatus Aphodocola excrementigallinarum sp. nov.}

Candidatus Aphodocola excrementigallinarum (ex.cre.men.ti.gal.li.na'rum. L. neut. $\mathrm{n}$.

678 excrementum excrement; L. fem. n. gallina hen; N.L. gen. n. excrementigallinarum of hen

679 excrement)

680

681

A bacterial species identified by metagenomic analyses. This species includes all bacteria with

682

683

684

685 genomes that show $\geq 95 \%$ average nucleotide identity (ANI) to the type genome, which has been assigned the MAG ID CHK193-30670 and which is available via NCBI BioSample SAMN15817049. The GC content of the type genome is $27.74 \%$ and the genome length is 1.2 Mbp.

686

687 
689 Candidatus Aphodomonas (Aph.o.d.omo'nas. Gr. fem. n. aphodos dung; L. fem. n. monas a 690 monad; N.L. fem. n. Aphodomonas a microbe associated with faeces)

691

692

693

694

695

696

697

698

699

700

701

702

703

704

705

706

707

708

709

710

711

712

713

714

715

716

717

718

719

720

721

722

723

724

725

726

727

728

729

730

731

732

733

734

A bacterial genus identified by metagenomic analyses. The genus includes all bacteria with genomes that show $\geq 60 \%$ average amino acid identity (AAI) to the type genome from the type species Aphodomonas merdavium. This is a name for the alphanumeric GTDB genus SFFS01. This genus has been assigned by GTDB-Tk v1.3.0 working on GTDB Release 05-RS95 (Chaumeil et al., 2019; Parks et al., 2020) to the order Christensenellales and to the family CAG74.

\section{Description of Candidatus Aphodomonas merdavium sp. nov.}

Candidatus Aphodomonas merdavium (merd.a'vi.um. L. fem. n. merda faeces; L. fem. n. avis bird; N.L. gen. n. merdavium of bird faeces)

A bacterial species identified by metagenomic analyses. This species includes all bacteria with genomes that show $\geq 95 \%$ average nucleotide identity (ANI) to the type genome, which has been assigned the MAG ID ChiGjej2B2-35035 and which is available via NCBI BioSample SAMN15817117. The GC content of the type genome is $59.45 \%$ and the genome length is 2.1 Mbp.

\section{Description of Candidatus Aphodomorpha gen. nov.}

Candidatus Aphodomorpha (Aph.o.d.o.mor'pha. Gr. fem. n. aphodos dung; Gr. fem. n. morphe a form, shape; N.L. fem. n. Aphodomorpha a microbe associated with faeces)

A bacterial genus identified by metagenomic analyses. The genus includes all bacteria with genomes that show $\geq 60 \%$ average amino acid identity (AAI) to the type genome from the type species Aphodomorpha intestinavium. This is a name for the alphanumeric GTDB genus UMGS1241. This genus has been assigned by GTDB-Tk v1.3.0 working on GTDB Release 05RS95 (Chaumeil et al., 2019; Parks et al., 2020) to the order Christensenellales and to the family $C A G-138$.

\section{Description of Candidatus Aphodomorpha intestinavium sp. nov.}

Candidatus Aphodomorpha intestinavium (in.tes.tin.a'vi.um. L. neut. n. intestinum gut; L. fem. n. avis bird; N.L. gen. n. intestinavium of the gut of birds)

A bacterial species identified by metagenomic analyses. This species includes all bacteria with genomes that show $\geq 95 \%$ average nucleotide identity (ANI) to the type genome, which has been assigned the MAG ID ChiGjej2B2-16831 and which is available via NCBI BioSample SAMN15817204. This is a new name for the alphanumeric GTDB species sp900550525. The GC content of the type genome is $68.13 \%$ and the genome length is $1.6 \mathrm{Mbp}$.

\section{Description of Candidatus Aphodoplasma gen. nov.}


735 Candidatus Aphodoplasma (Aph.o.d.o.plas'ma. Gr. fem. n. aphodos dung; Gr. neut. n. plasma a

736 form; N.L. neut. n. Aphodoplasma a microbe associated with faeces)

737

738

739

740

741

742

743

744

745

746

747

748

749

750

751

752

753

754

755

756

757

758

759

760

761

762

763

764

765

766

767

768

769

770

771

772

773

774

775

776

777

778

779

A bacterial genus identified by metagenomic analyses. The genus includes all bacteria with genomes that show $\geq 60 \%$ average amino acid identity (AAI) to the type genome from the type species Aphodoplasma excrementigallinarum. This is a name for the alphanumeric GTDB genus UMGS1253. This genus has been assigned by GTDB-Tk v1.3.0 working on GTDB Release 05RS95 (Chaumeil et al., 2019; Parks et al., 2020) to the order Monoglobales_A and to the family UMGS1253.

\section{Description of Candidatus Aphodoplasma excrementigallinarum sp. nov.}

Candidatus Aphodoplasma excrementigallinarum (ex.cre.men.ti.gal.li.na'rum. L. neut. $\mathrm{n}$. excrementum excrement; L. fem. n. gallina hen; N.L. gen. n. excrementigallinarum of hen excrement)

A bacterial species identified by metagenomic analyses. This species includes all bacteria with genomes that show $\geq 95 \%$ average nucleotide identity (ANI) to the type genome, which has been assigned the MAG ID 4920 and which is available via NCBI BioSample SAMN15817155. The GC content of the type genome is $54.59 \%$ and the genome length is $1.8 \mathrm{Mbp}$.

\section{Description of Candidatus Aphodosoma gen. nov.}

Candidatus Aphodosoma (Aph.o.d.o.so'ma. Gr. fem. n. aphodos dung; Gr. neut. n. soma a body; N.L. neut. n. Aphodosoma a microbe associated with faeces)

A bacterial genus identified by metagenomic analyses. The genus includes all bacteria with genomes that show $\geq 60 \%$ average amino acid identity (AAI) to the type genome from the type species Aphodosoma intestinipullorum. This is a name for the alphanumeric GTDB genus SFVR01. This genus has been assigned by GTDB-Tk v1.3.0 working on GTDB Release 05RS95 (Chaumeil et al., 2019; Parks et al., 2020) to the order Bacteroidales and to the family Paludibacteraceae.

\section{Description of Candidatus Aphodosoma intestinipullorum sp. nov.}

Candidatus Aphodosoma intestinipullorum (in.tes.ti.ni.pul.lo'rum. L. neut. n. intestinum gut; L. masc. n. pullus a young chicken; N.L. gen. n. intestinipullorum of the gut of young chickens)

A bacterial species identified by metagenomic analyses. This species includes all bacteria with genomes that show $\geq 95 \%$ average nucleotide identity (ANI) to the type genome, which has been assigned the MAG ID 3924 and which is available via NCBI BioSample SAMN15817132. The $\mathrm{GC}$ content of the type genome is $52.56 \%$ and the genome length is $2.4 \mathrm{Mbp}$.

\section{Description of Candidatus Aphodousia gen. nov.}


780 Candidatus Aphodousia (Aph.o.d.ou'si.a. Gr. fem. n. aphodos dung; Gr. fem. n. ousia an

781

782

783

784

785

786

787

788

789

790

791

792

793

794

795

796

797

798

799

800

801

802

803

804

805

806

807

808

809

810

811

812

813

814

815

816

817

818

819

820

821

822

823

824

essence; N.L. fem. n. Aphodousia a microbe associated with faeces)

A bacterial genus identified by metagenomic analyses. The genus includes all bacteria with genomes that show $\geq 60 \%$ average amino acid identity (AAI) to the type genome from the type species Aphodousia faecavium. This is a name for the alphanumeric GTDB genus CAG-521. This genus has been assigned by GTDB-Tk v1.3.0 working on GTDB Release 05-RS95 (Chaumeil et al., 2019; Parks et al., 2020) to the order Burkholderiales and to the family Burkholderiaceae.

\section{Description of Candidatus Aphodousia faecalis sp. nov. Candidatus Aphodousia faecalis (fae.ca'lis. L. fem. adj. faecalis of faeces)}

A bacterial species identified by metagenomic analyses. This species includes all bacteria with genomes that show $\geq 95 \%$ average nucleotide identity (ANI) to the type genome, which has been assigned the MAG ID ChiW13-1064 and which is available via NCBI BioSample SAMN15817170. This is a new name for the alphanumeric GTDB species sp000437635. The $\mathrm{GC}$ content of the type genome is $47.35 \%$ and the genome length is $1.7 \mathrm{Mbp}$.

\section{Description of Candidatus Aphodousia faecavium sp. nov.}

Candidatus Aphodousia faecavium (faec.a'vi.um. L. fem. n. faex, faecis excrement; L. fem. n. avis bird; N.L. gen. n. faecavium of bird faeces)

A bacterial species identified by metagenomic analyses. This species includes all bacteria with genomes that show $\geq 95 \%$ average nucleotide identity (ANI) to the type genome, which has been assigned the MAG ID 10345 and which is available via NCBI BioSample SAMN15817126. The GC content of the type genome is $48.23 \%$ and the genome length is $1.7 \mathrm{Mbp}$.

\section{Description of Candidatus Aphodousia faecigallinarum sp. nov.}

Candidatus Aphodousia faecigallinarum (fae.ci.gal.li.na'rum. L. fem. n. faex, faecis excrement; L. fem. n. gallina hen; N.L. gen. n. faecigallinarum of hen faeces)

A bacterial species identified by metagenomic analyses. This species includes all bacteria with genomes that show $\geq 95 \%$ average nucleotide identity (ANI) to the type genome, which has been assigned the MAG ID 7463 and which is available via NCBI BioSample SAMN15817137. The GC content of the type genome is $48.37 \%$ and the genome length is $1.5 \mathrm{Mbp}$.

\section{Description of Candidatus Aphodousia faecipullorum sp. nov.}

Candidatus Aphodousia faecipullorum (fae.ci.pul.lo'rum. L. fem. n. faex, faecis excrement; L. masc. n. pullus a young chicken; N.L. gen. n. faecipullorum of young chicken faeces) 
825 A bacterial species identified by metagenomic analyses. This species includes all bacteria with 826 genomes that show $\geq 95 \%$ average nucleotide identity (ANI) to the type genome, which has been 827 assigned the MAG ID CHK135-12538 and which is available via NCBI BioSample

828 SAMN15817146. The GC content of the type genome is $48.08 \%$ and the genome length is 1.8

829 Mbp.

830

831

832

833

Description of Candidatus Aphodousia gallistercoris sp. nov. Candidatus Aphodousia gallistercoris (gal.li.ster'co.ris. L. masc. $\mathrm{n}$ gallus chicken; L. neut. $\mathrm{n}$.

834

835

836

837

838 stercus dung; N.L. gen. n. gallistercoris of chicken faeces)

A bacterial species identified by metagenomic analyses. This species includes all bacteria with genomes that show $\geq 95 \%$ average nucleotide identity (ANI) to the type genome, which has been assigned the MAG ID CHK121-301 and which is available via NCBI BioSample

839 SAMN15817147. The GC content of the type genome is $52.58 \%$ and the genome length is 1.8 840 Mbp.

841

842

843

844

845

846

847

848

849

850

851

852

853

854

855

856

857

858

859

860

861

862

863

864

865

866

867

868

869

\section{Description of Candidatus Aphodovivens gen. nov.}

Candidatus Aphodovivens (Aph.o.d.o.viv'ens. Gr. fem. n. aphodos dung; N.L. pres. part. vivens living; N.L. fem. n. Aphodovivens a microbe associated with faeces)

A bacterial genus identified by metagenomic analyses. The genus includes all bacteria with genomes that show $\geq 60 \%$ average amino acid identity (AAI) to the type genome from the type species Aphodovivens avicola. This is a name for the alphanumeric GTDB genus UMGS1293. This genus has been assigned by GTDB-Tk v1.3.0 working on GTDB Release 05-RS95 (Chaumeil et al., 2019; Parks et al., 2020) to the order Coriobacteriales and to the family Eggerthellaceae.

\section{Description of Candidatus Aphodovivens avicola sp. nov.}

Candidatus Aphodovivens avicola (a.vi'co.la. L. fem. n. avis bird; L. suff. -cola inhabitant of; N.L. n. avicola inhabitant of birds)

A bacterial species identified by metagenomic analyses. This species includes all bacteria with genomes that show $\geq 95 \%$ average nucleotide identity (ANI) to the type genome, which has been assigned the MAG ID ChiGjej6B6-21069 and which is available via NCBI BioSample SAMN15817067. The GC content of the type genome is $65.54 \%$ and the genome length is 2.2 Mbp.

\section{Description of Candidatus Aphodovivens avistercoris sp. nov.}

Candidatus Aphodovivens avistercoris (a.vi.ster'co.ris. L. fem. n. avis bird; L. neut. n. stercus dung; N.L. gen. n. avistercoris of bird faeces) 
870 A bacterial species identified by metagenomic analyses. This species includes all bacteria with 871 genomes that show $\geq 95 \%$ average nucleotide identity (ANI) to the type genome, which has been 872 assigned the MAG ID ChiGjej5B5-3278 and which is available via NCBI BioSample

873 SAMN15817093. The GC content of the type genome is $66.86 \%$ and the genome length is 2.4

874 Mbp.

875

876

877

878

Description of Candidatus Aphodovivens excrementavium sp. nov.

Candidatus Aphodovivens excrementavium (ex.cre.ment.a'vi.um. L. neut. n. excrementum

879 excrement; L. fem. n. avis bird; N.L. gen. n. excrementavium of bird excrement)

880

881 A bacterial species identified by metagenomic analyses. This species includes all bacteria with

882

883 genomes that show $\geq 95 \%$ average nucleotide identity (ANI) to the type genome, which has been assigned the MAG ID ChiGjej2B2-30709 and which is available via NCBI BioSample

884

885

886

887

888

889

890

891

892

893

894

895

896

897

898

899

900

901 SAMN15817109. The GC content of the type genome is $58.74 \%$ and the genome length is 2.1 Mbp.

\section{Description of Candidatus Aquabacterium excrementipullorum sp. nov.} Candidatus Aquabacterium excrementipullorum (ex.cre.men.ti.pul.lo'rum. L. neut. n. excrementum excrement; L. masc. n. pullus a young chicken; N.L. gen. n. excrementipullorum of young chicken excrement)

A bacterial species identified by metagenomic analyses. This species includes all bacteria with genomes that show $\geq 95 \%$ average nucleotide identity (ANI) to the type genome, which has been assigned the MAG ID ChiHile3-4534 and which is available via NCBI BioSample SAMN15816783. The GC content of the type genome is $67.11 \%$ and the genome length is 4.7 Mbp.

\section{Description of Candidatus Atopostipes pullistercoris sp. nov.} Candidatus Atopostipes pullistercoris (pul.li.ster'co.ris. L. masc. n. pullus a young chicken; L.

902

903

904

905

906

907

908

909

910

911

Description of Candidatus Avacholeplasma gen. nov.

912 Candidatus Avacholeplasma (Av.a.cho.le.plas'ma. L. fem. n. avis bird; N.L. neut. n.

913 Acholeplasma a genus name; N.L. neut n. Aviacholeplasma a genus related to the genus

914 Acholeplasma but distinct from it and found in poultry)

915 
916 A bacterial genus identified by metagenomic analyses. The genus includes all bacteria with 917 genomes that show $\geq 60 \%$ average amino acid identity (AAI) to the type genome from the type 918 species Avacholeplasma faecigallinarum. This genus has been assigned by GTDB-Tk v1.3.0 919 working on GTDB Release 05-RS95 (Chaumeil et al., 2019; Parks et al., 2020) to the order 920 Acholeplasmatales and to the family Anaeroplasmataceae.

921

922

923

924

925

926

927

928

929

930

931

932

933

934

935

936

937

938

939

940

941

942

943

944

945

946

Description of Candidatus Avacholeplasma faecigallinarum sp. nov.

Candidatus Avacholeplasma faecigallinarum (fae.ci.gal.li.na'rum. L. fem. n. faex, faecis

excrement; L. fem. n. gallina hen; N.L. gen. n. faecigallinarum of hen faeces)

A bacterial species identified by metagenomic analyses. This species includes all bacteria with genomes that show $\geq 95 \%$ average nucleotide identity (ANI) to the type genome, which has been assigned the MAG ID 3263 and which is available via NCBI BioSample SAMN15816972. The $\mathrm{GC}$ content of the type genome is $29.88 \%$ and the genome length is $1.3 \mathrm{Mbp}$.

\section{Description of Candidatus Avacidaminococcus gen. nov.}

Candidatus Avacidaminococcus (Av.a.cid.a.mi.no.coc'cus. L. fem. n. avis bird; N.L. masc. n. Acidaminococcus a genus name; N.L. masc. n. Avacidaminococcus a genus related to the genus Acidaminococcus but distinct from it and found in poultry)

A bacterial genus identified by metagenomic analyses. The genus includes all bacteria with genomes that show $\geq 60 \%$ average amino acid identity (AAI) to the type genome from the type species Avacidaminococcus intestinavium. This genus has been assigned by GTDB-Tk v1.3.0 working on GTDB Release 05-RS95 (Chaumeil et al., 2019; Parks et al., 2020) to the order Acidaminococcales and to the family Acidaminococcaceae.

\section{Description of Candidatus Avacidaminococcus intestinavium sp. nov.}

Candidatus Avacidaminococcus intestinavium (in.tes.tin.a'vi.um. L. neut. n. intestinum gut; L.

947

948

A bacterial species identified by metagenomic analyses. This species includes all bacteria with genomes that show $\geq 95 \%$ average nucleotide identity (ANI) to the type genome, which has been assigned the MAG ID CHK160-1198 and which is available via NCBI BioSample SAMN15816987. The GC content of the type genome is $37.45 \%$ and the genome length is 1.6 Mbp.

\section{Description of Candidatus Avamphibacillus gen. nov.}

Candidatus Avamphibacillus (Av.am.phi.ba.cil'lus. L. fem. n. avis bird; N.L. masc. n. Amphibacillus a genus name; N.L. masc. n. Avamphibacillus a genus related to the genus Amphibacillus but distinct from it and found in poultry) 
961 A bacterial genus identified by metagenomic analyses. The genus includes all bacteria with 962 genomes that show $\geq 60 \%$ average amino acid identity (AAI) to the type genome from the type 963 species Avamphibacillus intestinigallinarum. This genus has been assigned by GTDB-Tk v1.3.0 964 working on GTDB Release 05-RS95 (Chaumeil et al., 2019; Parks et al., 2020) to the order 965 Bacillales and to the family Amphibacillaceae.

966

967

968

969

970

971

972

973

974

975

976

977

Description of Candidatus Avamphibacillus intestinigallinarum sp. nov.

Candidatus Avamphibacillus intestinigallinarum (in.tes.ti.ni.gal.li.na'rum. L. neut. n. intestinum gut; L. fem. n. gallina hen; N.L. gen. n. intestinigallinarum of the gut of the hens)

A bacterial species identified by metagenomic analyses. This species includes all bacteria with genomes that show $\geq 95 \%$ average nucleotide identity (ANI) to the type genome, which has been assigned the MAG ID CHK125-3527 and which is available via NCBI BioSample

SAMN15816959. The GC content of the type genome is $36.77 \%$ and the genome length is 2.0 Mbp.

978

979

\section{Description of Candidatus Avanaerovorax gen. nov.}

980

Candidatus Avanaerovorax (Av.an.a.e.ro.vo'rax. L. fem. n. avis bird; N.L. masc. n. Anaerovorax

981

a genus name; N.L. masc. n. Avanaerovorax a genus related to the genus Anaerovorax but

982

983

984

985

986

987

988

989

990

991

992

993

994

995

996

997

998

999 distinct from it and found in poultry)

A bacterial genus identified by metagenomic analyses. The genus includes all bacteria with genomes that show $\geq 60 \%$ average amino acid identity (AAI) to the type genome from the type species Avanaerovorax faecigallinarum. This genus has been assigned by GTDB-Tk v1.3.0 working on GTDB Release 05-RS95 (Chaumeil et al., 2019; Parks et al., 2020) to the order Peptostreptococcales and to the family Anaerovoracaceae.

\section{Description of Candidatus Avanaerovorax faecigallinarum sp. nov.}

Candidatus Avanaerovorax faecigallinarum (fae.ci.gal.li.na'rum. L. fem. n. faex, faecis excrement; L. fem. n. gallina hen; N.L. gen. n. faecigallinarum of hen faeces)

A bacterial species identified by metagenomic analyses. This species includes all bacteria with genomes that show $\geq 95 \%$ average nucleotide identity (ANI) to the type genome, which has been assigned the MAG ID Gambia13-1450 and which is available via NCBI BioSample SAMN15816994. The GC content of the type genome is $48.68 \%$ and the genome length is 1.8 Mbp.

\section{Description of Candidatus Aveggerthella gen. nov.}

1003 Candidatus Aveggerthella (Av.eg.ger.thel'la. L. fem. n. avis bird; N.L. fem. n. Eggerthella a 1004 genus name; N.L. fem. n. Aveggerthella a genus related to the genus Eggerthella but distinct 1005 from it and found in poultry)

1006 
1007 A bacterial genus identified by metagenomic analyses. The genus includes all bacteria with 1008 genomes that show $\geq 60 \%$ average amino acid identity (AAI) to the type genome from the type 1009 species Avieggerthella excrementigallinarum. This genus has been assigned by GTDB-Tk v1.3.0 1010 working on GTDB Release 05-RS95 (Chaumeil et al., 2019; Parks et al., 2020) to the order 1011 Coriobacteriales and to the family Eggerthellaceae.

1012

1013

1014

1015

Description of Candidatus Aveggerthella excrementigallinarum sp. nov.

1016

1017

1018

1019

1020

1021

1022

1023

1024

1025

1026

1027

Candidatus Aveggerthella excrementigallinarum (ex.cre.men.ti.gal.li.na'rum. L. neut. n. excrementum excrement; L. fem. n. gallina hen; N.L. gen. n. excrementigallinarum of hen excrement)

A bacterial species identified by metagenomic analyses. This species includes all bacteria with genomes that show $\geq 95 \%$ average nucleotide identity (ANI) to the type genome, which has been assigned the MAG ID ChiGjej4B4-3573 and which is available via NCBI BioSample SAMN15816976. The GC content of the type genome is $65.93 \%$ and the genome length is 2.0 Mbp.

1028

1029

1030

1031

1032

\section{Description of Candidatus Aveggerthella stercoripullorum sp. nov.}

Candidatus Aveggerthella stercoripullorum (ster.co.ri.pul.lo'rum. L. neut. n. stercus dung; L. masc. n. pullus a young chicken; N.L. gen. n. stercoripullorum of the faceces of young chickens)

A bacterial species identified by metagenomic analyses. This species includes all bacteria with genomes that show $\geq 95 \%$ average nucleotide identity (ANI) to the type genome, which has been assigned the MAG ID ChiGjej1B1-2707 and which is available via NCBI BioSample

1033

1034 SAMN15816950. The GC content of the type genome is $61.50 \%$ and the genome length is 2.1 Mbp.

1035

1036

Description of Candidatus Avelusimicrobium gen. nov.

1038 Candidatus Avelusimicrobium (Av.e.lu.si.mi.cro'bi.um. L. fem. n. avis bird; N.L. neut. n. Elusimicrobium a genus name; N.L. neut. n. Avelusimicrobium a genus related to the genus Elusimicrobium but distinct from it and found in poultry)

A bacterial genus identified by metagenomic analyses. The genus includes all bacteria with genomes that show $\geq 60 \%$ average amino acid identity (AAI) to the type genome from the type species Avielusimicrobium excrementipullorum. This genus has been assigned by GTDB-Tk v1.3.0 working on GTDB Release 05-RS95 (Chaumeil et al., 2019; Parks et al., 2020) to the order Elusimicrobiales and to the family Elusimicrobiaceae.

1047

1048

\section{Description of Candidatus Avelusimicrobium excrementipullorum sp. nov.}


1053

1054

1055

1056

1057

1058

1059

1060

1061

1062

1063

1064

1065

1066

1067

1068

1069

1070

1071

1072

1073

1074

1075

1076

1077

1078

1079

1080

1081

1082

1083

1084

1085

1086

1087

1088

1089

1090

1091

1092

1093

1094

1095

1096

1097

1098

A bacterial species identified by metagenomic analyses. This species includes all bacteria with genomes that show $\geq 95 \%$ average nucleotide identity (ANI) to the type genome, which has been assigned the MAG ID CHK136-6324 and which is available via NCBI BioSample

SAMN15817002. The GC content of the type genome is $53.46 \%$ and the genome length is 1.3 Mbp.

\section{Description of Candidatus Avibacteroides gen. nov.}

Candidatus Avibacteroides (A.vi.bac.te.ro'i.des. L. fem. n. avis bird; N.L. masc. n. Bacteroides a genus name; N.L. masc. n. Avibacteroides a genus related to the genus Bacteroides but distinct from it and found in poultry)

A bacterial genus identified by metagenomic analyses. The genus includes all bacteria with genomes that show $\geq 60 \%$ average amino acid identity (AAI) to the type genome from the type species Avibacteroides excrementipullorum. This genus has been assigned by GTDB-Tk v1.3.0 working on GTDB Release 05-RS95 (Chaumeil et al., 2019; Parks et al., 2020) to the order Bacteroidales and to the family Bacteroidaceae.

\section{Description of Candidatus Avibacteroides avistercoris sp. nov.}

Candidatus Avibacteroides avistercoris (a.vi.ster'co.ris. L. fem. n. avis bird; L. neut. n. stercus dung; N.L. gen. n. avistercoris of bird faeces)

A bacterial species identified by metagenomic analyses. This species includes all bacteria with genomes that show $\geq 95 \%$ average nucleotide identity (ANI) to the type genome, which has been assigned the MAG ID MalCec1-1739 and which is available via NCBI BioSample SAMN15816974. The GC content of the type genome is $53.14 \%$ and the genome length is 2.2 Mbp.

\section{Description of Candidatus Avibacteroides excrementipullorum sp. nov.} Candidatus Avibacteroides excrementipullorum (ex.cre.men.ti.pul.lo'rum. L. neut. n. excrementum excrement; L. masc. n. pullus a young chicken; N.L. gen. n. excrementipullorum of young chicken excrement)

A bacterial species identified by metagenomic analyses. This species includes all bacteria with genomes that show $\geq 95 \%$ average nucleotide identity (ANI) to the type genome, which has been assigned the MAG ID ChiHjej12B11-16860 and which is available via NCBI BioSample SAMN15816958. The GC content of the type genome is $47.79 \%$ and the genome length is 2.2 Mbp.

\section{Description of Candidatus Avibacteroides faecavium sp. nov.} Candidatus Avibacteroides faecavium (faec.a'vi.um. L. fem. n. faex, faecis excrement; L. fem. n. avis bird; N.L. gen. n. faecavium of bird faeces) 
1099

1100

1101

1102

1103

1104

1105

1106

1107

1108

1109

1110

1111

1112

1113

1114

1115

1116

1117

1118

1119

1120

1121

1122

1123

1124

1125

1126

1127

1128

1129

1130

1131

1132

1133

1134

1135

1136

1137

1138

1139

1140

1141

1142

1143

1144

A bacterial species identified by metagenomic analyses. This species includes all bacteria with genomes that show $\geq 95 \%$ average nucleotide identity (ANI) to the type genome, which has been assigned the MAG ID 3702 and which is available via NCBI BioSample SAMN15816980. The GC content of the type genome is $55.42 \%$ and the genome length is $2.1 \mathrm{Mbp}$.

\section{Description of Candidatus Avichristensenella gen. nov.}

Candidatus Avichristensenella (A.vi.chris.ten.sen.el'la. L. fem. n. avis bird; N.L. fem. n. Christensenella a genus name; N.L. fem. n. Avichristensenella a genus related to the genus Christensenella but distinct from it and found in poultry)

A bacterial genus identified by metagenomic analyses. The genus includes all bacteria with genomes that show $\geq 60 \%$ average amino acid identity (AAI) to the type genome from the type species Avichristensenella intestinipullorum. This genus has been assigned by GTDB-Tk v1.3.0 working on GTDB Release 05-RS95 (Chaumeil et al., 2019; Parks et al., 2020) to the order Christensenellales and to the family $C A G-74$.

\section{Description of Candidatus Avichristensenella intestinipullorum sp. nov.}

Candidatus Avichristensenella intestinipullorum (in.tes.ti.ni.pul.lo'rum. L. neut. n. intestinum gut; L. masc. n. pullus a young chicken; N.L. gen. n. intestinipullorum of the gut of young chickens)

A bacterial species identified by metagenomic analyses. This species includes all bacteria with genomes that show $\geq 95 \%$ average nucleotide identity (ANI) to the type genome, which has been assigned the MAG ID ChiHile30-977 and which is available via NCBI BioSample SAMN15816947. The GC content of the type genome is $63.80 \%$ and the genome length is 2.3 Mbp.

\section{Description of Candidatus Avidehalobacter gen. nov.}

Candidatus Avidehalobacter (A.vi.de.ha.lo.bac'ter. L. fem. n. avis bird; N.L. masc. n. Dehalobacter a genus name; N.L. masc. n. Avidehalobacter a genus related to the genus Dehalobacter but distinct from it and found in poultry)

A bacterial genus identified by metagenomic analyses. The genus includes all bacteria with genomes that show $\geq 60 \%$ average amino acid identity (AAI) to the type genome from the type species Avidehalobacter gallistercoris. This genus has been assigned by GTDB-Tk v1.3.0 working on GTDB Release 05-RS95 (Chaumeil et al., 2019; Parks et al., 2020) to the order $U B A 4068$ and to the family $U B A 5755$.

\section{Description of Candidatus Avidehalobacter gallistercoris sp. nov.}

Candidatus Avidehalobacter gallistercoris (gal.li.ster'co.ris. L. masc. n gallus chicken; L. neut. n. stercus dung; N.L. gen. n. gallistercoris of chicken faeces) 
A bacterial species identified by metagenomic analyses. This species includes all bacteria with genomes that show $\geq 95 \%$ average nucleotide identity (ANI) to the type genome, which has been assigned the MAG ID 2830 and which is available via NCBI BioSample SAMN15816981. The GC content of the type genome is $52.20 \%$ and the genome length is $1.4 \mathrm{Mbp}$.

1156

1157

1158

1159

\section{Description of Candidatus Avidesulfovibrio gen. nov.}

Candidatus Avidesulfovibrio (A.vi.de.sul.fo.vi'bri.o. L. fem. n. avis bird; N.L. masc. n. Desulfovibrio a genus name; N.L. masc. n. Avidesulfovibrio a genus related to the genus Desulfovibrio but distinct from it and found in poultry)

A bacterial genus identified by metagenomic analyses. The genus includes all bacteria with genomes that show $\geq 60 \%$ average amino acid identity (AAI) to the type genome from the type species Avidesulfovibrio excrementigallinarum. This genus has been assigned by GTDB-Tk v1.3.0 working on GTDB Release 05-RS95 (Chaumeil et al., 2019; Parks et al., 2020) to the order Desulfovibrionales and to the family Desulfovibrionaceae.

\section{Description of Candidatus Avidesulfovibrio excrementigallinarum sp. nov.} Candidatus Avidesulfovibrio excrementigallinarum (ex.cre.men.ti.gal.li.na'rum. L. neut. n. excrementum excrement; L. fem. n. gallina hen; N.L. gen. n. excrementigallinarum of hen excrement)

A bacterial species identified by metagenomic analyses. This species includes all bacteria with genomes that show $\geq 95 \%$ average nucleotide identity (ANI) to the type genome, which has been assigned the MAG ID ChiHcec4-2777 and which is available via NCBI BioSample SAMN15816982. The GC content of the type genome is $60.70 \%$ and the genome length is 2.2 Mbp.

Description of Candidatus Avigastranaerophilus gen. nov. Gastranaerophilus a genus name; N.L. masc. n. Avigastranaerophilus a genus related to the genus Gastranaerophilus but distinct from it and found in poultry)

1180

A bacterial genus identified by metagenomic analyses. The genus includes all bacteria with genomes that show $\geq 60 \%$ average amino acid identity (AAI) to the type genome from the type species Avigastranaerophilus faecigallinarum. This genus has been assigned by GTDB-Tk v1.3.0 working on GTDB Release 05-RS95 (Chaumeil et al., 2019; Parks et al., 2020) to the order Gastranaerophilales and to the family Gastranaerophilaceae.

1190

\section{Description of Candidatus Avigastranaerophilus faecigallinarum sp. nov.} Candidatus Avigastranaerophilus faecigallinarum (fae.ci.gal.li.na'rum. L. fem. n. faex, faecis excrement; L. fem. n. gallina hen; N.L. gen. n. faecigallinarum of hen faeces) 
1191

1192

1193

1194

1195

1196

1197

1198

1199

1200

1201

1202

1203

1204

1205

1206

1207

1208

1209

1210

1211

1212

1213

1214

1215

1216

1217

1218

1219

1220

1221

1222

1223

1224

1225

1226

1227

1228

1229

1230

1231

1232

1233

1234

1235

1236

A bacterial species identified by metagenomic analyses. This species includes all bacteria with genomes that show $\geq 95 \%$ average nucleotide identity (ANI) to the type genome, which has been assigned the MAG ID 5572 and which is available via NCBI BioSample SAMN15816968. The $\mathrm{GC}$ content of the type genome is $29.33 \%$ and the genome length is $2.2 \mathrm{Mbp}$.

\section{Description of Candidatus Avilachnospira gen. nov.}

Candidatus Avilachnospira (A.vi.lach.no.spi'ra. L. fem. n. avis bird; N.L. fem. n. Lachnospira a genus name; N.L. fem. n. Avilachnospira a genus related to the genus Lachnospira but distinct from it and found in poultry)

A bacterial genus identified by metagenomic analyses. The genus includes all bacteria with genomes that show $\geq 60 \%$ average amino acid identity (AAI) to the type genome from the type species Avilachnospira avistercoris. This genus has been assigned by GTDB-Tk v1.3.0 working on GTDB Release 05-RS95 (Chaumeil et al., 2019; Parks et al., 2020) to the order

Lachnospirales and to the family Lachnospiraceae.

\section{Description of Candidatus Avilachnospira avicola sp. nov.}

Candidatus Avilachnospira avicola (a.vi'co.la. L. fem. n. avis bird; L. suff. -cola inhabitant of; N.L. n. avicola inhabitant of birds)

A bacterial species identified by metagenomic analyses. This species includes all bacteria with genomes that show $\geq 95 \%$ average nucleotide identity (ANI) to the type genome, which has been assigned the MAG ID ChiHecec3B27-5021 and which is available via NCBI BioSample SAMN15816990. The GC content of the type genome is $49.15 \%$ and the genome length is 1.6 Mbp.

\section{Description of Candidatus Avilachnospira avistercoris sp. nov.}

Candidatus Avilachnospira avistercoris (a.vi.ster'co.ris. L. fem. n. avis bird; L. neut. n. stercus dung; N.L. gen. n. avistercoris of bird faeces)

A bacterial species identified by metagenomic analyses. This species includes all bacteria with genomes that show $\geq 95 \%$ average nucleotide identity (ANI) to the type genome, which has been assigned the MAG ID ChiGjej5B5-15814 and which is available via NCBI BioSample SAMN15816991. The GC content of the type genome is $50.02 \%$ and the genome length is 1.6 Mbp.

\section{Description of Candidatus Avimonoglobus gen. nov.}

Candidatus Avimonoglobus (A.vi.mo.no.glo'bus. L. fem. n. avis bird; N.L. masc. n. Monoglobus a genus name; N.L. masc. n. Avimonoglobus a genus related to the genus Monoglobus but distinct from it and found in poultry)

Peer) reviewing PDF | (2020:12:55980:1:1:NEW 13 Jan 2021) 
1237 A bacterial genus identified by metagenomic analyses. The genus includes all bacteria with 1238 genomes that show $\geq 60 \%$ average amino acid identity (AAI) to the type genome from the type 1239 species Avimonoglobus intestinipullorum. This genus has been assigned by GTDB-Tk v1.3.0

Description of Candidatus Avimonoglobus intestinipullorum sp. nov. Candidatus Avimonoglobus intestinipullorum (in.tes.ti.ni.pul.lo'rum. L. neut. n. intestinum gut; L. masc. n. pullus a young chicken; N.L. gen. n. intestinipullorum of the gut of young chickens) working on GTDB Release 05-RS95 (Chaumeil et al., 2019; Parks et al., 2020) to the order Monoglobales_A and to the family UBA1381.

A bacterial species identified by metagenomic analyses. This species includes all bacteria with genomes that show $\geq 95 \%$ average nucleotide identity (ANI) to the type genome, which has been assigned the MAG ID ChiSjej4B22-9803 and which is available via NCBI BioSample

SAMN15816985. The GC content of the type genome is $51.95 \%$ and the genome length is 1.8 Mbp.

\section{Description of Candidatus Avimuribaculum gen. nov.}

Candidatus Avimuribaculum (A.vi.mu.ri.ba'cu.lum. L. fem. n. avis bird; N.L. neut. n. Muribaculum a genus name; N.L. neut. n. Avimuribaculum a genus related to the genus Muribaculum but distinct from it and found in poultry)

A bacterial genus identified by metagenomic analyses. The genus includes all bacteria with genomes that show $\geq 60 \%$ average amino acid identity (AAI) to the type genome from the type species Avimuribaculum pullicola. This genus has been assigned by GTDB-Tk v1.3.0 working on GTDB Release 05-RS95 (Chaumeil et al., 2019; Parks et al., 2020) to the order Bacteroidales and to the family Muribaculaceae.

\section{Description of Candidatus Avimuribaculum pullicola sp. nov.} Candidatus Avimuribaculum pullicola (pul.li'co.la. L. masc. n. pullus a young chicken; L. suff. cola inhabitant of; N.L. n. pullicola an inhabitant of young chickens)

A bacterial species identified by metagenomic analyses. This species includes all bacteria with genomes that show $\geq 95 \%$ average nucleotide identity (ANI) to the type genome, which has been assigned the MAG ID ChiHecec3B27-9160 and which is available via NCBI BioSample SAMN15816969. The GC content of the type genome is $47.58 \%$ and the genome length is 2.2 Mbp.

\section{Description of Candidatus Avipropionibacterium gen. nov.}

Candidatus Avipropionibacterium (A.vi.pro.pi.o.ni.bac.te'ri.um. L. fem. n. avis bird; N.L. neut. n. Propionibacterium a genus name; N.L. neut. n. Avipropionibacterium a genus related to the genus Propionibacterium but distinct from it and found in poultry) 
1283 A bacterial genus identified by metagenomic analyses. The genus includes all bacteria with 1284 genomes that show $\geq 60 \%$ average amino acid identity (AAI) to the type genome from the type

1285 species Avipropionibacterium avicola. This genus has been assigned by GTDB-Tk v1.3.0

1286 working on GTDB Release 05-RS95 (Chaumeil et al., 2019; Parks et al., 2020) to the order

1287 Propionibacteriales and to the family Propionibacteriaceae.

1288

1289

1290

1291

1292

1293

1294

1295

1296

1297

1298

1299

1300

1301

1302

1303

1304

1305

1306

1307

1308

1309

1310

1311

1312

1313

1314

1315

1316

1317

1318

1319

1320

1321

1322

1323

1324

1325

1326

1327

\section{Description of Candidatus Avipropionibacterium avicola sp. nov.}

Candidatus Avipropionibacterium avicola (a.vi'co.la. L. fem. n. avis bird; L. suff. -cola inhabitant of; N.L. n. avicola inhabitant of birds)

A bacterial species identified by metagenomic analyses. This species includes all bacteria with genomes that show $\geq 95 \%$ average nucleotide identity (ANI) to the type genome, which has been assigned the MAG ID ChiGjej1B1-24693 and which is available via NCBI BioSample

SAMN15816979. The GC content of the type genome is $69.14 \%$ and the genome length is 3.2 Mbp.

\section{Description of Candidatus Avirikenella gen. nov.}

Candidatus Avirikenella (A.vi.ri.ke.nel'la. L. fem. n. avis bird; N.L. fem. n. Rikenella a genus name; N.L. fem. n. Avirikenella a genus related to the genus Rikenella but distinct from it and found in poultry)

A bacterial genus identified by metagenomic analyses. The genus includes all bacteria with genomes that show $\geq 60 \%$ average amino acid identity (AAI) to the type genome from the type species Avirikenella pullistercoris. This genus has been assigned by GTDB-Tk v1.3.0 working on GTDB Release 05-RS95 (Chaumeil et al., 2019; Parks et al., 2020) to the order Bacteroidales and to the family Rikenellaceae.

\section{Description of Candidatus Avirikenella pullistercoris sp. nov.}

Candidatus Avirikenella pullistercoris (pul.li.ster'co.ris. L. masc. n. pullus a young chicken; L. neut. n. stercus dung; N.L. gen. n. pullistercoris of young chicken faeces)

A bacterial species identified by metagenomic analyses. This species includes all bacteria with genomes that show $\geq 95 \%$ average nucleotide identity (ANI) to the type genome, which has been assigned the MAG ID 9321 and which is available via NCBI BioSample SAMN15816960. The $\mathrm{GC}$ content of the type genome is $41.91 \%$ and the genome length is $1.9 \mathrm{Mbp}$.

\section{Description of Candidatus Avisuccinivibrio gen. nov.}

Candidatus Avisuccinivibrio (A.vi.suc.ci.ni.vi'bri.o. L. fem. n. avis bird; N.L. masc. n. Succinivibrio a genus name; N.L. masc. n. Avisuccinivibrio a genus related to the genus Succinivibrio but distinct from it and found in poultry) 
1328 A bacterial genus identified by metagenomic analyses. The genus includes all bacteria with 1329 genomes that show $\geq 60 \%$ average amino acid identity (AAI) to the type genome from the type 1330 species Avisuccinivibrio stercorigallinarum. This genus has been assigned by GTDB-Tk v1.3.0 1331 working on GTDB Release 05-RS95 (Chaumeil et al., 2019; Parks et al., 2020) to the order 1332 Enterobacterales and to the family Succinivibrionaceae.

\section{Description of Candidatus Avisuccinivibrio pullicola sp. nov.}

Candidatus Avisuccinivibrio pullicola (pul.li'co.la. L. masc. n. pullus a young chicken; L. suff. cola inhabitant of; N.L. n. pullicola an inhabitant of young chickens)

A bacterial species identified by metagenomic analyses. This species includes all bacteria with genomes that show $\geq 95 \%$ average nucleotide identity (ANI) to the type genome, which has been assigned the MAG ID 3820 and which is available via NCBI BioSample SAMN15816999. The $\mathrm{GC}$ content of the type genome is $55.94 \%$ and the genome length is $2.4 \mathrm{Mbp}$.

\section{Description of Candidatus Avisuccinivibrio stercorigallinarum sp. nov.}

Candidatus Avisuccinivibrio stercorigallinarum (ster.co.ri.gal.li.na'rum. L. neut. n. stercus dung; L. fem. n. gallina hen; N.L. gen. n. stercorigallinarum of hen faeces)

A bacterial species identified by metagenomic analyses. This species includes all bacteria with genomes that show $\geq 95 \%$ average nucleotide identity (ANI) to the type genome, which has been assigned the MAG ID 17213 and which is available via NCBI BioSample SAMN15817000. The GC content of the type genome is $54.49 \%$ and the genome length is $2.4 \mathrm{Mbp}$.

\section{Description of Candidatus Avitreponema gen. nov.}

Candidatus Avitreponema (A.vi.tre.po.ne'ma. L. fem. n. avis bird; N.L. neut. n. Treponema a genus name; N.L. neut. n. Avitreponema a genus related to the genus Treponema but distinct from it and found in poultry)

A bacterial genus identified by metagenomic analyses. The genus includes all bacteria with genomes that show $\geq 60 \%$ average amino acid identity (AAI) to the type genome from the type species Avitreponema avistercoris. This genus has been assigned by GTDB-Tk v1.3.0 working on GTDB Release 05-RS95 (Chaumeil et al., 2019; Parks et al., 2020) to the order

Treponematales and to the family Treponemataceae.

\section{Description of Candidatus Avitreponema avistercoris sp. nov.}

Candidatus Avitreponema avistercoris (a.vi.ster'co.ris. L. fem. n. avis bird; L. neut. n. stercus dung; N.L. gen. n. avistercoris of bird faeces)

A bacterial species identified by metagenomic analyses. This species includes all bacteria with genomes that show $\geq 95 \%$ average nucleotide identity (ANI) to the type genome, which has been 
1373 assigned the MAG ID B3-4054 and which is available via NCBI BioSample SAMN15816977.

1374 The GC content of the type genome is $55.36 \%$ and the genome length is $1.9 \mathrm{Mbp}$.

1375

1376

1377

1378

1379

1380

1381

1382

1383

1384

1385

1386

1387

1388

1389

Description of Candidatus Avoscillospira gen. nov.

Candidatus Avoscillospira (Av.os.cil.lo.spi'ra. L. fem. n. avis bird; N.L. fem. n. Oscillospira a genus name; N.L. fem. n. Avoscillospira a genus related to the genus Oscillospira but distinct from it and found in poultry)

A bacterial genus identified by metagenomic analyses. The genus includes all bacteria with genomes that show $\geq 60 \%$ average amino acid identity (AAI) to the type genome from the type species Avioscillospira stercorigallinarum. This genus has been assigned by GTDB-Tk v1.3.0 working on GTDB Release 05-RS95 (Chaumeil et al., 2019; Parks et al., 2020) to the order Oscillospirales and to the family Oscillospiraceae.

\section{Description of Candidatus Avoscillospira avicola sp. nov.}

1390

1391 Candidatus Avoscillospira avicola (a.vi'co.la. L. fem. n. avis bird; L. suff. -cola inhabitant of; N.L. n. avicola inhabitant of birds)

1392

A bacterial species identified by metagenomic analyses. This species includes all bacteria with genomes that show $\geq 95 \%$ average nucleotide identity (ANI) to the type genome, which has been assigned the MAG ID ChiBcec15-4380 and which is available via NCBI BioSample SAMN15816934. The GC content of the type genome is $61.76 \%$ and the genome length is 2.5 Mbp.

Description of Candidatus Avoscillospira avistercoris sp. nov. dung; N.L. gen. n. avistercoris of bird faeces)

A bacterial species identified by metagenomic analyses. This species includes all bacteria with genomes that show $\geq 95 \%$ average nucleotide identity (ANI) to the type genome, which has been assigned the MAG ID ChiBcec16-1751 and which is available via NCBI BioSample SAMN15816964. The GC content of the type genome is $58.00 \%$ and the genome length is 2.4 Mbp.

\section{Description of Candidatus Avoscillospira stercorigallinarum sp. nov.}

1412 Candidatus Avoscillospira stercorigallinarum (ster.co.ri.gal.li.na'rum. L. neut. n. stercus dung; L. fem. n. gallina hen; N.L. gen. n. stercorigallinarum of hen faeces) genomes that show $\geq 95 \%$ average nucleotide identity (ANI) to the type genome, which has been assigned the MAG ID ChiSjej2B20-13462 and which is available via NCBI BioSample 
1418 SAMN15816948. The GC content of the type genome is $63.01 \%$ and the genome length is 2.2 1419 Mbp.

1420

1421

1422

1423

Description of Candidatus Avoscillospira stercoripullorum sp. nov. Candidatus Avoscillospira stercoripullorum (ster.co.ri.pul.lo'rum. L. neut. n. stercus dung; L. masc. n. pullus a young chicken; N.L. gen. n. stercoripullorum of the faceces of young chickens)

Description of Candidatus Bacteroides avicola sp. nov.

A bacterial species identified by metagenomic analyses. This species includes all bacteria with genomes that show $\geq 95 \%$ average nucleotide identity (ANI) to the type genome, which has been assigned the MAG ID ChiHjej9B8-7071 and which is available via NCBI BioSample SAMN15816951. The GC content of the type genome is $60.69 \%$ and the genome length is 1.9 Mbp. Candidatus Bacteroides avicola (a.vi'co.la. L. fem. n. avis bird; L. suff. -cola inhabitant of; N.L. n. avicola inhabitant of birds)

A bacterial species identified by metagenomic analyses. This species includes all bacteria with genomes that show $\geq 95 \%$ average nucleotide identity (ANI) to the type genome, which has been assigned the MAG ID ChiHjej12B11-9795 and which is available via NCBI BioSample SAMN15816830. This is a new name for the alphanumeric GTDB species sp002160055. The $\mathrm{GC}$ content of the type genome is $50.12 \%$ and the genome length is $3.0 \mathrm{Mbp}$.

1445

\section{Description of Candidatus Bacteroides intestinigallinarum sp. nov.}

A bacterial species identified by metagenomic analyses. This species includes all bacteria with genomes that show $\geq 95 \%$ average nucleotide identity (ANI) to the type genome, which has been assigned the MAG ID 2926 and which is available via NCBI BioSample SAMN15816831. This is a new name for the alphanumeric GTDB species sp003463205. The GC content of the type 
Description of Candidatus Bacteroides intestinipullorum sp. nov.

1467

Candidatus Bacteroides intestinipullorum (in.tes.ti.ni.pul.lo'rum. L. neut. n. intestinum gut; L.

1468 masc. n. pullus a young chicken; N.L. gen. n. intestinipullorum of the gut of young chickens)

A bacterial species identified by metagenomic analyses. This species includes all bacteria with genomes that show $\geq 95 \%$ average nucleotide identity (ANI) to the type genome, which has been assigned the MAG ID B3-3758 and which is available via NCBI BioSample SAMN15816671. The GC content of the type genome is $53.99 \%$ and the genome length is $2.7 \mathrm{Mbp}$.

Description of Candidatus Bacteroides merdavium sp. nov.

Candidatus Bacteroides merdavium (merd.a'vi.um. L. fem. n. merda faeces; L. fem. n. avis bird; N.L. gen. n. merdavium of bird faeces)

A bacterial species identified by metagenomic analyses. This species includes all bacteria with genomes that show $\geq 95 \%$ average nucleotide identity (ANI) to the type genome, which has been assigned the MAG ID CHK118-2852 and which is available via NCBI BioSample SAMN15816687. The GC content of the type genome is $49.76 \%$ and the genome length is 2.9

$1484 \mathrm{Mbp}$.

\section{Description of Candidatus Bacteroides merdigallinarum sp. nov.}

1488 Candidatus Bacteroides merdigallinarum (mer.di.gal.li.na'rum. L. fem. n. merda faeces; L. fem. n. gallina hen; N.L. gen. n. merdigallinarum of hen faeces)

A bacterial species identified by metagenomic analyses. This species includes all bacteria with genomes that show $\geq 95 \%$ average nucleotide identity (ANI) to the type genome, which has been assigned the MAG ID ChiHjej9B8-1298 and which is available via NCBI BioSample SAMN15816694. The GC content of the type genome is $54.52 \%$ and the genome length is 2.7

Description of Candidatus Bacteroides merdipullorum sp. nov. Candidatus Bacteroides merdipullorum (mer.di.pul.lo'rum. L. fem. n. merda faeces; L. masc. n. pullus a young chicken; N.L. gen. n. merdipullorum of the faeces of young chickens)

A bacterial species identified by metagenomic analyses. This species includes all bacteria with genomes that show $\geq 95 \%$ average nucleotide identity (ANI) to the type genome, which has been assigned the MAG ID ChiHjej12B11-24981 and which is available via NCBI BioSample SAMN15816699. The GC content of the type genome is $53.73 \%$ and the genome length is 2.5 Mbp. 
1510 Candidatus Bacteroides pullicola (pul.li'co.la. L. masc. n. pullus a young chicken; L. suff. -cola

1511 inhabitant of; N.L. n. pullicola an inhabitant of young chickens)

1512

1513

1514

1515

1516

1517

1518

1519

A bacterial species identified by metagenomic analyses. This species includes all bacteria with genomes that show $\geq 95 \%$ average nucleotide identity (ANI) to the type genome, which has been assigned the MAG ID Gambia2-208 and which is available via NCBI BioSample SAMN15816704. The GC content of the type genome is $55.18 \%$ and the genome length is 2.7 Mbp.

\section{Description of Candidatus Bariatricus faecipullorum sp. nov.} Candidatus Bariatricus faecipullorum (fae.ci.pul.lo'rum. L. fem. n. faex, faecis excrement; L. masc. n. pullus a young chicken; N.L. gen. n. faecipullorum of young chicken faeces)

A bacterial species identified by metagenomic analyses. This species includes all bacteria with genomes that show $\geq 95 \%$ average nucleotide identity (ANI) to the type genome, which has been assigned the MAG ID 9095 and which is available via NCBI BioSample SAMN15816662. The $\mathrm{GC}$ content of the type genome is $51.54 \%$ and the genome length is $2.5 \mathrm{Mbp}$.

\section{Description of Candidatus Barnesiella excrementavium sp. nov.}

Candidatus Barnesiella excrementavium (ex.cre.ment.a'vi.um. L. neut. n. excrementum excrement; L. fem. n. avis bird; N.L. gen. n. excrementavium of bird excrement)

A bacterial species identified by metagenomic analyses. This species includes all bacteria with genomes that show $\geq 95 \%$ average nucleotide identity (ANI) to the type genome, which has been assigned the MAG ID 4398 and which is available via NCBI BioSample SAMN15816714. The $\mathrm{GC}$ content of the type genome is $52.73 \%$ and the genome length is $2.7 \mathrm{Mbp}$.

\section{Description of Candidatus Barnesiella excrementigallinarum sp. nov.}

Candidatus Barnesiella excrementigallinarum (ex.cre.men.ti.gal.li.na'rum. L. neut. n. excrementum excrement; L. fem. n. gallina hen; N.L. gen. n. excrementigallinarum of hen excrement)

A bacterial species identified by metagenomic analyses. This species includes all bacteria with genomes that show $\geq 95 \%$ average nucleotide identity (ANI) to the type genome, which has been assigned the MAG ID CHK169-14362 and which is available via NCBI BioSample SAMN15816739. The GC content of the type genome is $47.04 \%$ and the genome length is 2.6 Mbp.

\section{Description of Candidatus Barnesiella excrementipullorum sp. nov.}

Candidatus Barnesiella excrementipullorum (ex.cre.men.ti.pul.lo'rum. L. neut. n. excrementum excrement; L. masc. n. pullus a young chicken; N.L. gen. n. excrementipullorum of young chicken excrement) 
A bacterial species identified by metagenomic analyses. This species includes all bacteria with

1558

1559 genomes that show $\geq 95 \%$ average nucleotide identity (ANI) to the type genome, which has been assigned the MAG ID ChiHjej12B11-16260 and which is available via NCBI BioSample SAMN15816862. This is a new name for the alphanumeric GTDB species sp900542255. The $\mathrm{GC}$ content of the type genome is $50.69 \%$ and the genome length is $2.1 \mathrm{Mbp}$.

1562

1563

1564

Description of Candidatus Barnesiella merdigallinarum sp. nov.

1565

Candidatus Barnesiella merdigallinarum (mer.di.gal.li.na'rum. L. fem. n. merda faeces; L. fem.

1566

1567 n. gallina hen; N.L. gen. n. merdigallinarum of hen faeces)

A bacterial species identified by metagenomic analyses. This species includes all bacteria with genomes that show $\geq 95 \%$ average nucleotide identity (ANI) to the type genome, which has been assigned the MAG ID CHK136-6590 and which is available via NCBI BioSample

1572 SAMN15816819. This is a new name for the alphanumeric GTDB species sp002159975. The

1573

1574 $\mathrm{GC}$ content of the type genome is $54.15 \%$ and the genome length is $2.7 \mathrm{Mbp}$.

\section{Description of Candidatus Barnesiella merdipullorum sp. nov.}

Candidatus Barnesiella merdipullorum (mer.di.pul.lo'rum. L. fem. n. merda faeces; L. masc. n.

1577

1578

1579 pullus a young chicken; N.L. gen. n. merdipullorum of the faeces of young chickens)

A bacterial species identified by metagenomic analyses. This species includes all bacteria with genomes that show $\geq 95 \%$ average nucleotide identity (ANI) to the type genome, which has been assigned the MAG ID 5648 and which is available via NCBI BioSample SAMN15816849. This is a new name for the alphanumeric GTDB species sp002161555. The GC content of the type genome is $52.62 \%$ and the genome length is $2.6 \mathrm{Mbp}$.

\section{Description of Candidatus Bilophila faecipullorum sp. nov.}

1587 Candidatus Bilophila faecipullorum (fae.ci.pul.lo'rum. L. fem. n. faex, faecis excrement; L. masc. n. pullus a young chicken; N.L. gen. n. faecipullorum of young chicken faeces)

A bacterial species identified by metagenomic analyses. This species includes all bacteria with genomes that show $\geq 95 \%$ average nucleotide identity (ANI) to the type genome, which has been assigned the MAG ID ChiSxjej5B17-1746 and which is available via NCBI BioSample SAMN15816754. The GC content of the type genome is $63.22 \%$ and the genome length is 2.8 Mbp.

Description of Candidatus Blautia avicola sp. nov.

1598 Candidatus Blautia avicola (a.vi'co.la. L. fem. n. avis bird; L. suff. -cola inhabitant of; N.L. n. 1599 avicola inhabitant of birds) 
1601 A bacterial species identified by metagenomic analyses. This species includes all bacteria with 1602 genomes that show $\geq 95 \%$ average nucleotide identity (ANI) to the type genome, which has been 1603 assigned the MAG ID ChiBcec6-4105 and which is available via NCBI BioSample

1604 SAMN15816794. The GC content of the type genome is $46.40 \%$ and the genome length is 3.1

1605 Mbp.

1606

1607

1608

1609

1610

1611

1612

1613

1614

1615

1616

1617

1618

1619

1620

1621

1622

1623

1624

1625

1626

1627

1628

1629

1630

1631

1632

1633

1634

1635

1636

1637

1638

1639

1640

1641

1642

1643

1644

1645

1646

Description of Candidatus Blautia avistercoris sp. nov.

Candidatus Blautia avistercoris (a.vi.ster'co.ris. L. fem. n. avis bird; L. neut. n. stercus dung; N.L. gen. n. avistercoris of bird faeces)

A bacterial species identified by metagenomic analyses. This species includes all bacteria with genomes that show $\geq 95 \%$ average nucleotide identity (ANI) to the type genome, which has been assigned the MAG ID 5548 and which is available via NCBI BioSample SAMN15816924. This is a new name for the alphanumeric GTDB species sp002159835. Although GTDB has assigned this species to the genus it calls Blautia_A, this genus designation cannot be incorporated into a well-formed binomial, so in naming this species, we have used the current validly published name for the genus. The GC content of the type genome is $45.38 \%$ and the genome length is 2.5 Mbp.

\section{Description of Candidatus Blautia excrementigallinarum sp. nov.}

Candidatus Blautia excrementigallinarum (ex.cre.men.ti.gal.li.na'rum. L. neut. n. excrementum excrement; L. fem. n. gallina hen; N.L. gen. n. excrementigallinarum of hen excrement)

A bacterial species identified by metagenomic analyses. This species includes all bacteria with genomes that show $\geq 95 \%$ average nucleotide identity (ANI) to the type genome, which has been assigned the MAG ID ChiSjej6B24-370 and which is available via NCBI BioSample SAMN15816894. Although GTDB has assigned this species to the genus it calls Blautia_A, this genus designation cannot be incorporated into a well-formed binomial, so in naming this species, we have used the current validly published name for the genus. The GC content of the type genome is $49.44 \%$ and the genome length is $2.3 \mathrm{Mbp}$.

\section{Description of Candidatus Blautia excrementipullorum sp. nov.}

Candidatus Blautia excrementipullorum (ex.cre.men.ti.pul.lo'rum. L. neut. n. excrementum excrement; L. masc. n. pullus a young chicken; N.L. gen. n. excrementipullorum of young chicken excrement)

A bacterial species identified by metagenomic analyses. This species includes all bacteria with genomes that show $\geq 95 \%$ average nucleotide identity (ANI) to the type genome, which has been assigned the MAG ID CHK197-7439 and which is available via NCBI BioSample SAMN15816885. Although GTDB has assigned this species to the genus it calls Blautia_A, this genus designation cannot be incorporated into a well-formed binomial, so in naming this species, we have used the current validly published name for the genus. The GC content of the type genome is $46.79 \%$ and the genome length is $3.3 \mathrm{Mbp}$. 
Description of Candidatus Blautia faecavium sp. nov.

1650

1651

Candidatus Blautia faecavium (faec.a'vi.um. L. fem. n. faex, faecis excrement; L. fem. n. avis bird; N.L. gen. n. faecavium of bird faeces)

1652

1653

A bacterial species identified by metagenomic analyses. This species includes all bacteria with

1654

1655 genomes that show $\geq 95 \%$ average nucleotide identity (ANI) to the type genome, which has been assigned the MAG ID ChiSjej1B19-5720 and which is available via NCBI BioSample SAMN15816886. Although GTDB has assigned this species to the genus it calls Blautia_A, this genus designation cannot be incorporated into a well-formed binomial, so in naming this species, we have used the current validly published name for the genus. The GC content of the type genome is $45.35 \%$ and the genome length is $3.5 \mathrm{Mbp}$.

1661

\section{Description of Candidatus Blautia faecigallinarum sp. nov.}

1664

\section{Description of Candidatus Blautia faecipullorum sp. nov.}

1677 Candidatus Blautia faecipullorum (fae.ci.pul.lo'rum. L. fem. n. faex, faecis excrement; L. masc. n. pullus a young chicken; N.L. gen. n. faecipullorum of young chicken faeces)

A bacterial species identified by metagenomic analyses. This species includes all bacteria with genomes that show $\geq 95 \%$ average nucleotide identity (ANI) to the type genome, which has been assigned the MAG ID ChiSxjej6B18-2004 and which is available via NCBI BioSample SAMN15816906. Although GTDB has assigned this species to the genus it calls Blautia_A, this genus designation cannot be incorporated into a well-formed binomial, so in naming this species, we have used the current validly published name for the genus. The GC content of the type genome is $48.18 \%$ and the genome length is $3.5 \mathrm{Mbp}$. 
1692 A bacterial species identified by metagenomic analyses. This species includes all bacteria with 1693 genomes that show $\geq 95 \%$ average nucleotide identity (ANI) to the type genome, which has been 1694 assigned the MAG ID ChiSjej1B19-8411 and which is available via NCBI BioSample

1695 SAMN15816925. This is a new name for the alphanumeric GTDB species sp900542045.

1696 Although GTDB has assigned this species to the genus it calls Blautia_A, this genus designation 1697 cannot be incorporated into a well-formed binomial, so in naming this species, we have used the 1698 current validly published name for the genus. The GC content of the type genome is $48.96 \%$ and 1699 the genome length is $2.8 \mathrm{Mbp}$.

1700

1701

1702

1703

1704

1705

1706

1707

1708

1709

1710

1711

1712

1713

1714

1715

1716

1717

1718

1719

1720

1721

1722

1723

1724

1725

1726

1727

1728

1729

1730

1731

1732

1733

1734

1735

1736

\section{Description of Candidatus Blautia intestinavium sp. nov.}

Candidatus Blautia intestinavium (in.tes.tin.a'vi.um. L. neut. n. intestinum gut; L. fem. n. avis bird; N.L. gen. n. intestinavium of the gut of birds)

A bacterial species identified by metagenomic analyses. This species includes all bacteria with genomes that show $\geq 95 \%$ average nucleotide identity (ANI) to the type genome, which has been assigned the MAG ID CHK186-553 and which is available via NCBI BioSample SAMN15816890. Although GTDB has assigned this species to the genus it calls Blautia_A, this genus designation cannot be incorporated into a well-formed binomial, so in naming this species, we have used the current validly published name for the genus. The GC content of the type genome is $47.27 \%$ and the genome length is $2.8 \mathrm{Mbp}$.

\section{Description of Candidatus Blautia intestinigallinarum sp. nov.} Candidatus Blautia intestinigallinarum (in.tes.ti.ni.gal.li.na'rum. L. neut. n. intestinum gut; L. fem. n. gallina hen; N.L. gen. n. intestinigallinarum of the gut of the hens)

A bacterial species identified by metagenomic analyses. This species includes all bacteria with genomes that show $\geq 95 \%$ average nucleotide identity (ANI) to the type genome, which has been assigned the MAG ID CHK186-9876 and which is available via NCBI BioSample SAMN15816891. Although GTDB has assigned this species to the genus it calls Blautia_A, this genus designation cannot be incorporated into a well-formed binomial, so in naming this species, we have used the current validly published name for the genus. The GC content of the type genome is $46.77 \%$ and the genome length is $2.7 \mathrm{Mbp}$.

\section{Description of Candidatus Blautia intestinipullorum sp. nov.}

Candidatus Blautia intestinipullorum (in.tes.ti.ni.pul.lo'rum. L. neut. n. intestinum gut; L. masc. n. pullus a young chicken; N.L. gen. n. intestinipullorum of the gut of young chickens)

A bacterial species identified by metagenomic analyses. This species includes all bacteria with genomes that show $\geq 95 \%$ average nucleotide identity (ANI) to the type genome, which has been assigned the MAG ID ChiW16-4312 and which is available via NCBI BioSample

SAMN15816892. Although GTDB has assigned this species to the genus it calls Blautia_A, this genus designation cannot be incorporated into a well-formed binomial, so in naming this species, 
1737 we have used the current validly published name for the genus. The GC content of the type

1738

1739

1740

1741

1742

1743

1744

1745

1746

1747

1748

1749

1750

1751

1752

1753

1754

1755

1756

1757

1758

1759

1760

1761

1762

1763

1764

1765

1766

1767

1768

1769

1770

1771

1772

1773

1774

1775

1776

1777

1778

1779

1780

1781

1782

genome is $47.05 \%$ and the genome length is $2.4 \mathrm{Mbp}$.

\section{Description of Candidatus Blautia merdavium sp. nov.}

Candidatus Blautia merdavium (merd.a'vi.um. L. fem. n. merda faeces; L. fem. n. avis bird; N.L. gen. n. merdavium of bird faeces)

A bacterial species identified by metagenomic analyses. This species includes all bacteria with genomes that show $\geq 95 \%$ average nucleotide identity (ANI) to the type genome, which has been assigned the MAG ID ChiBcec2-3848 and which is available via NCBI BioSample SAMN15816633. The GC content of the type genome is $48.60 \%$ and the genome length is 3.3 Mbp.

\section{Description of Candidatus Blautia merdigallinarum sp. nov.} Candidatus Blautia merdigallinarum (mer.di.gal.li.na'rum. L. fem. n. merda faeces; L. fem. n. gallina hen; N.L. gen. n. merdigallinarum of hen faeces)

A bacterial species identified by metagenomic analyses. This species includes all bacteria with genomes that show $\geq 95 \%$ average nucleotide identity (ANI) to the type genome, which has been assigned the MAG ID ChiSxjej6B18-287 and which is available via NCBI BioSample SAMN15816815. This is a new name for the alphanumeric GTDB species sp900543715. The $\mathrm{GC}$ content of the type genome is $45.18 \%$ and the genome length is $3.3 \mathrm{Mbp}$.

\section{Description of Candidatus Blautia merdipullorum sp. nov.}

Candidatus Blautia merdipullorum (mer.di.pul.lo'rum. L. fem. n. merda faeces; L. masc. n. pullus a young chicken; N.L. gen. n. merdipullorum of the faeces of young chickens)

A bacterial species identified by metagenomic analyses. This species includes all bacteria with genomes that show $\geq 95 \%$ average nucleotide identity (ANI) to the type genome, which has been assigned the MAG ID 17058 and which is available via NCBI BioSample SAMN15816655. The $\mathrm{GC}$ content of the type genome is $45.05 \%$ and the genome length is $3.2 \mathrm{Mbp}$.

\section{Description of Candidatus Blautia ornithocaccae sp. nov.}

Candidatus Blautia ornithocaccae (or.ni.tho.cac'cae. Gr. masc. or fem. n. ornis, ornithos bird Gr. fem. n. kakke faeces; N.L. gen. n. ornithocaccae of bird faeces)

A bacterial species identified by metagenomic analyses. This species includes all bacteria with genomes that show $\geq 95 \%$ average nucleotide identity (ANI) to the type genome, which has been assigned the MAG ID ChiBcec1-3711 and which is available via NCBI BioSample SAMN15816880. This is a new name for the alphanumeric GTDB species sp002161285. The $\mathrm{GC}$ content of the type genome is $44.79 \%$ and the genome length is $3.1 \mathrm{Mbp}$. 
Description of Candidatus Blautia pullicola sp. nov.

1786

1787

1788

1789

1790

1791

1792

1793

1794

Candidatus Blautia pullicola (pul.li'co.la. L. masc. n. pullus a young chicken; L. suff. -cola inhabitant of; N.L. n. pullicola an inhabitant of young chickens)

A bacterial species identified by metagenomic analyses. This species includes all bacteria with genomes that show $\geq 95 \%$ average nucleotide identity (ANI) to the type genome, which has been assigned the MAG ID 1068 and which is available via NCBI BioSample SAMN15816689. The $\mathrm{GC}$ content of the type genome is $45.62 \%$ and the genome length is $3.0 \mathrm{Mbp}$.

1795

Description of Candidatus Blautia pullistercoris sp. nov.

1796

1797

1798 Candidatus Blautia pullistercoris (pul.li.ster'co.ris. L. masc. n. pullus a young chicken; L. neut. n. stercus dung; N.L. gen. n. pullistercoris of young chicken faeces)

1799

A bacterial species identified by metagenomic analyses. This species includes all bacteria with genomes that show $\geq 95 \%$ average nucleotide identity (ANI) to the type genome, which has been assigned the MAG ID ChiHjej12B11-1927 and which is available via NCBI BioSample SAMN15816618. The GC content of the type genome is $45.73 \%$ and the genome length is 3.2 $1802 \mathrm{Mbp}$.

1803

1804

Description of Candidatus Blautia stercoravium sp. nov.

1806 Candidatus Blautia stercoravium (ster.cor.a'vi.um. L. neut. n. stercus dung; L. fem. n. avis bird; N.L. gen. n. stercoravium of bird faeces)

A bacterial species identified by metagenomic analyses. This species includes all bacteria with genomes that show $\geq 95 \%$ average nucleotide identity (ANI) to the type genome, which has been assigned the MAG ID 3268 and which is available via NCBI BioSample SAMN15816738. The $\mathrm{GC}$ content of the type genome is $44.17 \%$ and the genome length is $2.7 \mathrm{Mbp}$.

1813

1814

\section{Description of Candidatus Blautia stercorigallinarum sp. nov.}

1816 Candidatus Blautia stercorigallinarum (ster.co.ri.gal.li.na'rum. L. neut. n. stercus dung; L. fem. 1817 n. gallina hen; N.L. gen. n. stercorigallinarum of hen faeces)

1818

1819

1820

1821

1822

1823

1824

1825

1826

1827

1828
A bacterial species identified by metagenomic analyses. This species includes all bacteria with genomes that show $\geq 95 \%$ average nucleotide identity (ANI) to the type genome, which has been assigned the MAG ID CHK195-9823 and which is available via NCBI BioSample SAMN15816627. The GC content of the type genome is $45.83 \%$ and the genome length is 3.1 Mbp.

\section{Description of Candidatus Blautia stercoripullorum sp. nov.}

Candidatus Blautia stercoripullorum (ster.co.ri.pul.lo'rum. L. neut. n. stercus dung; L. masc. n. pullus a young chicken; N.L. gen. n. stercoripullorum of the faceces of young chickens) 
1829

1830

1831

1832

1833

1834

1835

1836

1837

1838

1839

1840

1841

1842

1843

1844

1845

1846

1847

1848

1849

1850

1851

1852

1853

1854

1855

1856

1857

1858

1859

1860

1861

1862

1863

1864

1865

1866

1867

1868

1869

1870

1871

1872

1873

1874

A bacterial species identified by metagenomic analyses. This species includes all bacteria with genomes that show $\geq 95 \%$ average nucleotide identity (ANI) to the type genome, which has been assigned the MAG ID ChiW19-6364 and which is available via NCBI BioSample SAMN15816793. The GC content of the type genome is $44.78 \%$ and the genome length is 3.1 Mbp.

\section{Description of Candidatus Borkfalkia avicola sp. nov.}

Candidatus Borkfalkia avicola (a.vi'co.la. L. fem. n. avis bird; L. suff. -cola inhabitant of; N.L. n. avicola inhabitant of birds)

A bacterial species identified by metagenomic analyses. This species includes all bacteria with genomes that show $\geq 95 \%$ average nucleotide identity (ANI) to the type genome, which has been assigned the MAG ID CHK192-19661 and which is available via NCBI BioSample SAMN15816606. The GC content of the type genome is $58.92 \%$ and the genome length is 1.7 Mbp.

\section{Description of Candidatus Borkfalkia avistercoris sp. nov.}

Candidatus Borkfalkia avistercoris (a.vi.ster'co.ris. L. fem. n. avis bird; L. neut. n. stercus dung; N.L. gen. n. avistercoris of bird faeces)

A bacterial species identified by metagenomic analyses. This species includes all bacteria with genomes that show $\geq 95 \%$ average nucleotide identity (ANI) to the type genome, which has been assigned the MAG ID CHK187-5294 and which is available via NCBI BioSample SAMN15816607. The GC content of the type genome is $53.99 \%$ and the genome length is 1.7 Mbp.

\section{Description of Candidatus Borkfalkia excrementavium sp. nov.}

Candidatus Borkfalkia excrementavium (ex.cre.ment.a'vi.um. L. neut. n. excrementum excrement; L. fem. n. avis bird; N.L. gen. n. excrementavium of bird excrement)

A bacterial species identified by metagenomic analyses. This species includes all bacteria with genomes that show $\geq 95 \%$ average nucleotide identity (ANI) to the type genome, which has been assigned the MAG ID CHK199-9574 and which is available via NCBI BioSample SAMN15816608. The GC content of the type genome is $52.76 \%$ and the genome length is 1.6 Mbp.

\section{Description of Candidatus Borkfalkia excrementigallinarum sp. nov.}

Candidatus Borkfalkia excrementigallinarum (ex.cre.men.ti.gal.li.na'rum. L. neut. n. excrementum excrement; L. fem. n. gallina hen; N.L. gen. n. excrementigallinarum of hen excrement) 
1875 A bacterial species identified by metagenomic analyses. This species includes all bacteria with 1876 genomes that show $\geq 95 \%$ average nucleotide identity (ANI) to the type genome, which has been 1877 assigned the MAG ID 1345 and which is available via NCBI BioSample SAMN15816609. The 1878 GC content of the type genome is $53.42 \%$ and the genome length is $1.9 \mathrm{Mbp}$.

1879

1880

1881

1882

1883

1884

1885

1886

1887

1888

1889

1890

1891

1892

1893

1894

1895

1896

1897

1898

1899

1900

1901

1902

1903

1904

1905

1906

1907

1908

1909

1910

1911

1912

1913

1914

1915

1916

1917

1918

1919

1920

\section{Description of Candidatus Borkfalkia excrementipullorum sp. nov.}

Candidatus Borkfalkia excrementipullorum (ex.cre.men.ti.pul.lo'rum. L. neut. n. excrementum excrement; L. masc. n. pullus a young chicken; N.L. gen. n. excrementipullorum of young chicken excrement)

A bacterial species identified by metagenomic analyses. This species includes all bacteria with genomes that show $\geq 95 \%$ average nucleotide identity (ANI) to the type genome, which has been assigned the MAG ID CHK192-2667 and which is available via NCBI BioSample

SAMN15816611. The GC content of the type genome is $55.63 \%$ and the genome length is 1.6 Mbp.

\section{Description of Candidatus Borkfalkia faecavium sp. nov.}

Candidatus Borkfalkia faecavium (faec.a'vi.um. L. fem. n. faex, faecis excrement; L. fem. n. avis bird; N.L. gen. n. faecavium of bird faeces)

A bacterial species identified by metagenomic analyses. This species includes all bacteria with genomes that show $\geq 95 \%$ average nucleotide identity (ANI) to the type genome, which has been assigned the MAG ID 2189 and which is available via NCBI BioSample SAMN15816731. The $\mathrm{GC}$ content of the type genome is $58.98 \%$ and the genome length is $1.7 \mathrm{Mbp}$.

\section{Description of Candidatus Borkfalkia faecigallinarum sp. nov.}

Candidatus Borkfalkia faecigallinarum (fae.ci.gal.li.na'rum. L. fem. n. faex, faecis excrement; L. fem. n. gallina hen; N.L. gen. n. faecigallinarum of hen faeces)

A bacterial species identified by metagenomic analyses. This species includes all bacteria with genomes that show $\geq 95 \%$ average nucleotide identity (ANI) to the type genome, which has been assigned the MAG ID 26628 and which is available via NCBI BioSample SAMN15816617. The $\mathrm{GC}$ content of the type genome is $62.49 \%$ and the genome length is $1.6 \mathrm{Mbp}$.

\section{Description of Candidatus Borkfalkia faecipullorum sp. nov.} Candidatus Borkfalkia faecipullorum (fae.ci.pul.lo'rum. L. fem. n. faex, faecis excrement; L. masc. n. pullus a young chicken; N.L. gen. n. faecipullorum of young chicken faeces)

A bacterial species identified by metagenomic analyses. This species includes all bacteria with genomes that show $\geq 95 \%$ average nucleotide identity (ANI) to the type genome, which has been assigned the MAG ID 811 and which is available via NCBI BioSample SAMN15816621. The $\mathrm{GC}$ content of the type genome is $54.29 \%$ and the genome length is $1.9 \mathrm{Mbp}$. 
Description of Candidatus Borkfalkia stercoripullorum sp. nov.

1925

1926

1927

Candidatus Borkfalkia stercoripullorum (ster.co.ri.pul.lo'rum. L. neut. n. stercus dung; L. masc. n. pullus a young chicken; N.L. gen. n. stercoripullorum of the faceces of young chickens)

1928

A bacterial species identified by metagenomic analyses. This species includes all bacteria with genomes that show $\geq 95 \%$ average nucleotide identity (ANI) to the type genome, which has been assigned the MAG ID CHK196-13738 and which is available via NCBI BioSample

1929 SAMN15816588. The GC content of the type genome is $55.22 \%$ and the genome length is 1.8 Mbp.

1932

1933

1934

Description of Candidatus Brachybacterium intestinipullorum sp. nov.

1935

Candidatus Brachybacterium intestinipullorum (in.tes.ti.ni.pul.lo'rum. L. neut. n. intestinum gut;

1936

1937

1938

L. masc. n. pullus a young chicken; N.L. gen. n. intestinipullorum of the gut of young chickens)

1939

A bacterial species identified by metagenomic analyses. This species includes all bacteria with genomes that show $\geq 95 \%$ average nucleotide identity (ANI) to the type genome, which has been assigned the MAG ID CHK130-7132 and which is available via NCBI BioSample SAMN15816812. This is a new name for the alphanumeric GTDB species sp003711805. The $\mathrm{GC}$ content of the type genome is $72.59 \%$ and the genome length is $3.5 \mathrm{Mbp}$.

\section{Description of Candidatus Brachybacterium merdavium sp. nov.}

Candidatus Brachybacterium merdavium (merd.a'vi.um. L. fem. n. merda faeces; L. fem. n. avis bird; N.L. gen. n. merdavium of bird faeces)

A bacterial species identified by metagenomic analyses. This species includes all bacteria with genomes that show $\geq 95 \%$ average nucleotide identity (ANI) to the type genome, which has been assigned the MAG ID ChiHjej13B12-24818 and which is available via NCBI BioSample SAMN15816666. The GC content of the type genome is $70.22 \%$ and the genome length is 3.7 Mbp.

\section{Description of Candidatus Brachybacterium merdigallinarum sp. nov.}

1957 Candidatus Brachybacterium merdigallinarum (mer.di.gal.li.na'rum. L. fem. n. merda faeces; L. fem. n. gallina hen; N.L. gen. n. merdigallinarum of hen faeces)

A bacterial species identified by metagenomic analyses. This species includes all bacteria with genomes that show $\geq 95 \%$ average nucleotide identity (ANI) to the type genome, which has been assigned the MAG ID ChiHjej13B12-7362 and which is available via NCBI BioSample 
1967 Description of Candidatus Brevibacterium intestinavium sp. nov.

1968 Candidatus Brevibacterium intestinavium (in.tes.tin.a'vi.um. L. neut. n. intestinum gut; L. fem. n.

1969 avis bird; N.L. gen. n. intestinavium of the gut of birds)

1970

1971

1972

1973

1974

1975

1976

1977

1978

Description of Candidatus Brevibacterium intestinigallinarum sp. nov.

A bacterial species identified by metagenomic analyses. This species includes all bacteria with genomes that show $\geq 95 \%$ average nucleotide identity (ANI) to the type genome, which has been assigned the MAG ID 5295 and which is available via NCBI BioSample SAMN15816668. The $\mathrm{GC}$ content of the type genome is $66.76 \%$ and the genome length is $3.2 \mathrm{Mbp}$.

1979

1980

1981

1982

1983 Candidatus Brevibacterium intestinigallinarum (in.tes.ti.ni.gal.li.na'rum. L. neut. n. intestinum gut; L. fem. n. gallina hen; N.L. gen. n. intestinigallinarum of the gut of the hens)

A bacterial species identified by metagenomic analyses. This species includes all bacteria with genomes that show $\geq 95 \%$ average nucleotide identity (ANI) to the type genome, which has been assigned the MAG ID CHK132-2174 and which is available via NCBI BioSample

1984

1985 SAMN15816673. The GC content of the type genome is $70.54 \%$ and the genome length is 2.7 Mbp.

1986

1987

Description of Candidatus Butyricicoccus avicola sp. nov.

1989 Candidatus Butyricicoccus avicola (a.vi'co.la. L. fem. n. avis bird; L. suff. -cola inhabitant of; N.L. n. avicola inhabitant of birds)

A bacterial species identified by metagenomic analyses. This species includes all bacteria with genomes that show $\geq 95 \%$ average nucleotide identity (ANI) to the type genome, which has been assigned the MAG ID ChiSjej6B24-14740 and which is available via NCBI BioSample SAMN15816587. The GC content of the type genome is $59.67 \%$ and the genome length is 2.0 $1996 \mathrm{Mbp}$.

1997

Description of Candidatus Butyricicoccus avistercoris sp. nov.

2000 Candidatus Butyricicoccus avistercoris (a.vi.ster'co.ris. L. fem. n. avis bird; L. neut. n. stercus dung; N.L. gen. n. avistercoris of bird faeces)

2002

2003

2004

2005

A bacterial species identified by metagenomic analyses. This species includes all bacteria with genomes that show $\geq 95 \%$ average nucleotide identity (ANI) to the type genome, which has been assigned the MAG ID CHK193-4272 and which is available via NCBI BioSample

2006

2007

2008

2009

2010 SAMN15816626. The GC content of the type genome is $35.60 \%$ and the genome length is 2.0 Mbp.

2011

\section{Description of Candidatus Butyricicoccus stercorigallinarum sp. nov.}

2012 L. fem. n. gallina hen; N.L. gen. n. stercorigallinarum of hen faeces) 
2013

2014

2015

2016

2017

2018

2019

2020

2021

2022

2023

2024

2025

2026

2027

2028

2029

2030

2031

2032

2033

2034

2035

2036

2037

2038

2039

2040

2041

2042

2043

2044

2045

2046

2047

2048

2049

2050

2051

2052

2053

2054

2055

2056

2057

2058

A bacterial species identified by metagenomic analyses. This species includes all bacteria with genomes that show $\geq 95 \%$ average nucleotide identity (ANI) to the type genome, which has been assigned the MAG ID ChiHecec2B26-12929 and which is available via NCBI BioSample SAMN15816912. Although GTDB has assigned this species to the genus it calls Butyricicoccus_A, this genus designation cannot be incorporated into a well-formed binomial, so in naming this species, we have used the current validly published name for the genus. The $\mathrm{GC}$ content of the type genome is $62.06 \%$ and the genome length is $2.1 \mathrm{Mbp}$.

\section{Description of Candidatus Butyricimonas faecavium sp. nov.}

Candidatus Butyricimonas faecavium (faec.a'vi.um. L. fem. n. faex, faecis excrement; L. fem. n. avis bird; N.L. gen. n. faecavium of bird faeces)

A bacterial species identified by metagenomic analyses. This species includes all bacteria with genomes that show $\geq 95 \%$ average nucleotide identity (ANI) to the type genome, which has been assigned the MAG ID CHK27-1272 and which is available via NCBI BioSample

SAMN15816676. The GC content of the type genome is $40.60 \%$ and the genome length is 3.6 Mbp.

\section{Description of Candidatus Caccalectryoclostridium gen. nov.}

Candidatus Caccalectryoclostridium (Cacc.a.lec.try.o.clos.tri'di.um. Gr. fem. n. kakke faeces; Gr. neut. n. alektryon chicken; N.L. neut. n. Clostridium a genus name; N.L. neut. n.

Caccalectryoclostridium a genus related to the genus Clostridium but distinct from it and found in poultry faeces)

A bacterial genus identified by metagenomic analyses. The genus includes all bacteria with genomes that show $\geq 60 \%$ average amino acid identity (AAI) to the type genome from the type species Caccalectryoclostridium excrementigallinarum. This genus has been assigned by GTDBTk v1.3.0 working on GTDB Release 05-RS95 (Chaumeil et al., 2019; Parks et al., 2020) to the order Christensenellales and to the family UBA3700.

\section{Description of Candidatus Caccalectryoclostridium excrementigallinarum sp. nov.}

Candidatus Caccalectryoclostridium excrementigallinarum (ex.cre.men.ti.gal.li.na'rum. L. neut. n. excrementum excrement; L. fem. n. gallina hen; N.L. gen. n. excrementigallinarum of hen excrement)

A bacterial species identified by metagenomic analyses. This species includes all bacteria with genomes that show $\geq 95 \%$ average nucleotide identity (ANI) to the type genome, which has been assigned the MAG ID 9366 and which is available via NCBI BioSample SAMN15816988. The $\mathrm{GC}$ content of the type genome is $54.72 \%$ and the genome length is $1.5 \mathrm{Mbp}$.

\section{Description of Candidatus Caccenecus gen. nov.}


2059 Candidatus Caccenecus (Caccen.e'cus. Gr. fem. n. kakke dung; Gr. masc. enoikos inhabitant;

2060 N.L. masc. n. Caccenecus a microbe associated with faeces)

2061

2062

2063

2064

2065

2066

2067

2068

2069

2070

2071

2072

2073

2074

2075

2076

2077

2078

2079

2080

2081

2082

2083

2084

2085

2086

2087

2088

2089

2090

2091

2092

2093

2094

2095

2096

2097

2098

2099

A bacterial genus identified by metagenomic analyses. The genus includes all bacteria with genomes that show $\geq 60 \%$ average amino acid identity (AAI) to the type genome from the type species Caccenecus avistercoris. This is a name for the alphanumeric GTDB genus CAG-776. This genus has been assigned by GTDB-Tk v1.3.0 working on GTDB Release 05-RS95 (Chaumeil et al., 2019; Parks et al., 2020) to the order $R F 39$ and to the family $C A G-1000$.

\section{Description of Candidatus Caccenecus avistercoris sp. nov.}

Candidatus Caccenecus avistercoris (a.vi.ster'co.ris. L. fem. n. avis bird; L. neut. n. stercus dung; N.L. gen. n. avistercoris of bird faeces)

A bacterial species identified by metagenomic analyses. This species includes all bacteria with genomes that show $\geq 95 \%$ average nucleotide identity (ANI) to the type genome, which has been assigned the MAG ID CHK193-13418 and which is available via NCBI BioSample SAMN15817030. The GC content of the type genome is $28.47 \%$ and the genome length is 1.3 Mbp.

\section{Description of Candidatus Caccocola gen. nov.}

Candidatus Caccocola (Cacc.o.co'la. Gr. fem. n. kakke dung; L. suff. -cola inhabitant of; N.L. fem. n. Caccocola a microbe associated with faeces)

A bacterial genus identified by metagenomic analyses. The genus includes all bacteria with genomes that show $\geq 60 \%$ average amino acid identity (AAI) to the type genome from the type species Caccocola faecigallinarum. This is a name for the alphanumeric GTDB genus An23. This genus has been assigned by GTDB-Tk v1.3.0 working on GTDB Release 05-RS95 (Chaumeil et al., 2019; Parks et al., 2020) to the order Synergistales and to the family Synergistaceae.

\section{Description of Candidatus Caccocola faecigallinarum sp. nov.} Candidatus Caccocola faecigallinarum (fae.ci.gal.li.na'rum. L. fem. n. faex, faecis excrement; L. fem. n. gallina hen; N.L. gen. n. faecigallinarum of hen faeces)

A bacterial species identified by metagenomic analyses. This species includes all bacteria with genomes that show $\geq 95 \%$ average nucleotide identity (ANI) to the type genome, which has been assigned the MAG ID ChiHjej11B10-3130 and which is available via NCBI BioSample SAMN15817215. This is a new name for the alphanumeric GTDB species sp900544635. The $\mathrm{GC}$ content of the type genome is $60.31 \%$ and the genome length is $2.3 \mathrm{Mbp}$.

\section{Description of Candidatus Caccocola faecipullorum sp. nov.}


2104 Candidatus Caccocola faecipullorum (fae.ci.pul.lo'rum. L. fem. n. faex, faecis excrement; L.

2105

2106

2107

2108

2109

2110

2111

2112

2113

2114

2115

2116

2117

2118

2119

2120

2121

2122

2123

2124

2125

2126

2127

2128

2129

2130

2131

2132

2133

2134

2135

2136

2137

2138

2139

2140

2141

2142

2143

2144

2145

2146

2147

2148

masc. n. pullus a young chicken; N.L. gen. n. faecipullorum of young chicken faeces)

A bacterial species identified by metagenomic analyses. This species includes all bacteria with genomes that show $\geq 95 \%$ average nucleotide identity (ANI) to the type genome, which has been assigned the MAG ID ChiHcec3-7374 and which is available via NCBI BioSample SAMN15817103. The GC content of the type genome is $59.08 \%$ and the genome length is 2.2 Mbp.

\section{Description of Candidatus Caccomonas gen. nov.}

Candidatus Caccomonas (Cacc.omo'nas. Gr. fem. n. kakke dung; L. fem. n. monas a monad; N.L. fem. n. Caccomonas a microbe associated with faeces)

A bacterial genus identified by metagenomic analyses. The genus includes all bacteria with genomes that show $\geq 60 \%$ average amino acid identity (AAI) to the type genome from the type species Caccomonas pullistercoris. This is a name for the alphanumeric GTDB genus CAG-617. This genus has been assigned by GTDB-Tk v1.3.0 working on GTDB Release 05-RS95 (Chaumeil et al., 2019; Parks et al., 2020) to the order Bacteroidales and to the family Bacteroidaceae.

\section{Description of Candidatus Caccomonas pullistercoris sp. nov.} Candidatus Caccomonas pullistercoris (pul.li.ster'co.ris. L. masc. n. pullus a young chicken; L. neut. n. stercus dung; N.L. gen. n. pullistercoris of young chicken faeces)

A bacterial species identified by metagenomic analyses. This species includes all bacteria with genomes that show $\geq 95 \%$ average nucleotide identity (ANI) to the type genome, which has been assigned the MAG ID 5345 and which is available via NCBI BioSample SAMN15817152. The GC content of the type genome is $59.45 \%$ and the genome length is $2.2 \mathrm{Mbp}$.

\section{Description of Candidatus Caccomorpha gen. nov.}

Candidatus Caccomorpha (Cacc.o.mor'pha. Gr. fem. n. kakke dung; Gr. fem. n. morphe a form, shape; N.L. fem. n. Caccomorpha a microbe associated with faeces)

A bacterial genus identified by metagenomic analyses. The genus includes all bacteria with genomes that show $\geq 60 \%$ average amino acid identity (AAI) to the type genome from the type species Caccomorpha excrementavium. This is a name for the alphanumeric GTDB genus SZUA-448. This genus has been assigned by GTDB-Tk v1.3.0 working on GTDB Release 05RS95 (Chaumeil et al., 2019; Parks et al., 2020) to the order Lachnospirales and to the family Lachnospiraceae.

\section{Description of Candidatus Caccomorpha excrementavium sp. nov.}


2149 Candidatus Caccomorpha excrementavium (ex.cre.ment.a'vi.um. L. neut. n. excrementum

2150

2151

2152

2153

2154

2155

2156

2157

2158

2159

2160

2161

2162

2163

2164

2165

2166

2167

2168

2169

2170

2171

2172

2173

2174

2175

2176

2177

2178

2179

2180

2181

2182

2183

2184

2185

2186

2187

2188

2189

2190

2191

2192

2193

2194

excrement; L. fem. n. avis bird; N.L. gen. n. excrementavium of bird excrement)

A bacterial species identified by metagenomic analyses. This species includes all bacteria with genomes that show $\geq 95 \%$ average nucleotide identity (ANI) to the type genome, which has been assigned the MAG ID 1999 and which is available via NCBI BioSample SAMN15817145. The $\mathrm{GC}$ content of the type genome is $50.75 \%$ and the genome length is $2.7 \mathrm{Mbp}$.

\section{Description of Candidatus Caccoplasma gen. nov.}

Candidatus Caccoplasma (Cacc.o.plas'ma. Gr. fem. n. kakke dung; Gr. neut. n. plasma a form; N.L. neut. n. Caccoplasma a microbe associated with faeces)

A bacterial genus identified by metagenomic analyses. The genus includes all bacteria with genomes that show $\geq 60 \%$ average amino acid identity (AAI) to the type genome from the type species Caccoplasma merdavium. This is a name for the alphanumeric GTDB genus UBA11471. This genus has been assigned by GTDB-Tk v1.3.0 working on GTDB Release 05-RS95 (Chaumeil et al., 2019; Parks et al., 2020) to the order Bacteroidales and to the family UBA11471.

\section{Description of Candidatus Caccoplasma intestinavium sp. nov.}

Candidatus Caccoplasma intestinavium (in.tes.tin.a'vi.um. L. neut. n. intestinum gut; L. fem. $\mathrm{n}$. avis bird; N.L. gen. $\mathrm{n}$. intestinavium of the gut of birds)

A bacterial species identified by metagenomic analyses. This species includes all bacteria with genomes that show $\geq 95 \%$ average nucleotide identity (ANI) to the type genome, which has been assigned the MAG ID 21143 and which is available via NCBI BioSample SAMN15817187. This is a new name for the alphanumeric GTDB species sp000434215. The GC content of the type genome is $45.83 \%$ and the genome length is $2.4 \mathrm{Mbp}$.

\section{Description of Candidatus Caccoplasma merdavium sp. nov.}

Candidatus Caccoplasma merdavium (merd.a'vi.um. L. fem. n. merda faeces; L. fem. n. avis bird; N.L. gen. n. merdavium of bird faeces)

A bacterial species identified by metagenomic analyses. This species includes all bacteria with genomes that show $\geq 95 \%$ average nucleotide identity (ANI) to the type genome, which has been assigned the MAG ID 6821 and which is available via NCBI BioSample SAMN15817190. This is a new name for the alphanumeric GTDB species sp900542765. The GC content of the type genome is $52.79 \%$ and the genome length is $2.4 \mathrm{Mbp}$.

\section{Description of Candidatus Caccoplasma merdipullorum sp. nov.}

Candidatus Caccoplasma merdipullorum (mer.di.pul.lo'rum. L. fem. n. merda faeces; L. masc. n. pullus a young chicken; N.L. gen. n. merdipullorum of the faeces of young chickens)

PeerJ reviewing PDF | (2020:12:55980:1:1:NEW 13 Jan 2021) 
2195

2196

2197

2198

2199

2200

2201

2202

2203

2204

2205

2206

2207

2208

2209

2210

2211

2212

2213

2214

2215

2216

2217

2218

2219

2220

2221

2222

2223

2224

2225

2226

2227

2228

2229

2230

2231

2232

2233

2234

2235

2236

2237

2238

2239

2240
A bacterial species identified by metagenomic analyses. This species includes all bacteria with genomes that show $\geq 95 \%$ average nucleotide identity (ANI) to the type genome, which has been assigned the MAG ID G3-4614 and which is available via NCBI BioSample SAMN15817135. The GC content of the type genome is $46.70 \%$ and the genome length is $2.0 \mathrm{Mbp}$.

\section{Description of Candidatus Caccopulliclostridium gen. nov.}

Candidatus Caccopulliclostridium (Cac.co.pul.li.clos.tri'di.um. Gr. fem. n. kakke faeces; L. masc. n. pullus a young chicken; N.L. neut. n. Clostridium a genus name; N.L. neut. n.

Caccopulliclostridium a genus related to the genus Clostridium but distinct from it and found in poultry faeces)

A bacterial genus identified by metagenomic analyses. The genus includes all bacteria with genomes that show $\geq 60 \%$ average amino acid identity (AAI) to the type genome from the type species Caccopulliclostridium gallistercoris. This genus was identified but not named by Glendinning et al. This genus has been assigned by GTDB-Tk v1.3.0 working on GTDB Release 05-RS95 (Chaumeil et al., 2019; Parks et al., 2020) to the order 4C28d-15 and to the family UBA1242.

\section{Description of Candidatus Caccopulliclostridium gallistercoris sp. nov.}

Candidatus Caccopulliclostridium gallistercoris (gal.li.ster'co.ris. L. masc. n gallus chicken; L. neut. n. stercus dung; N.L. gen. n. gallistercoris of chicken faeces)

A bacterial species identified by metagenomic analyses. This species includes all bacteria with genomes that show $\geq 95 \%$ average nucleotide identity (ANI) to the type genome, which has been assigned the MAG ID CHK186-9395 and which is available via NCBI BioSample SAMN15816943. The GC content of the type genome is $35.40 \%$ and the genome length is 1.0 Mbp.

\section{Description of Candidatus Caccosoma gen. nov.} Candidatus Caccosoma (Cacc.o.so'ma. Gr. fem. n. kakke dung; Gr. neut. n. soma a body; N.L. neut. n. Caccosoma a microbe associated with faeces)

A bacterial genus identified by metagenomic analyses. The genus includes all bacteria with genomes that show $\geq 60 \%$ average amino acid identity (AAI) to the type genome from the type species Caccosoma faecigallinarum. This is a name for the alphanumeric GTDB genus CAG631. This genus has been assigned by GTDB-Tk v1.3.0 working on GTDB Release 05-RS95 (Chaumeil et al., 2019; Parks et al., 2020) to the order RFN20 and to the family CAG-631.

\section{Description of Candidatus Caccosoma faecigallinarum sp. nov.}

Candidatus Caccosoma faecigallinarum (fae.ci.gal.li.na'rum. L. fem. n. faex, faecis excrement; L. fem. n. gallina hen; N.L. gen. n. faecigallinarum of hen faeces)

Peer] reviewing PDF | (2020:12:55980:1:1:NEW 13 Jan 2021) 
2241

2242

2243

2244

2245

2246

2247

2248

2249

2250

2251

2252

2253

2254

2255

2256

2257

2258

2259

2260

2261

2262

2263

2264

2265

2266

2267

2268

2269

2270

2271

2272

2273

2274

2275

2276

2277

2278

2279

2280

2281

2282

2283

2284

2285

2286

A bacterial species identified by metagenomic analyses. This species includes all bacteria with genomes that show $\geq 95 \%$ average nucleotide identity (ANI) to the type genome, which has been assigned the MAG ID 14508 and which is available via NCBI BioSample SAMN15817185. This is a new name for the alphanumeric GTDB species sp000433015. The GC content of the type genome is $30.38 \%$ and the genome length is $1.3 \mathrm{Mbp}$.

\section{Description of Candidatus Caccousia gen. nov.}

Candidatus Caccousia (Cacc.ou'si.a. Gr. fem. n. kakke dung; Gr. fem. n. ousia an essence; N.L. fem. n. Caccousia a microbe associated with faeces)

A bacterial genus identified by metagenomic analyses. The genus includes all bacteria with genomes that show $\geq 60 \%$ average amino acid identity (AAI) to the type genome from the type species Caccousia avicola. This is a name for the alphanumeric GTDB genus An200. This genus has been assigned by GTDB-Tk v1.3.0 working on GTDB Release 05-RS95 (Chaumeil et al., 2019; Parks et al., 2020) to the order Oscillospirales and to the family Acutalibacteraceae.

\section{Description of Candidatus Caccousia avicola sp. nov.}

Candidatus Caccousia avicola (a.vi'co.la. L. fem. n. avis bird; L. suff. -cola inhabitant of; N.L. n. avicola inhabitant of birds)

A bacterial species identified by metagenomic analyses. This species includes all bacteria with genomes that show $\geq 95 \%$ average nucleotide identity (ANI) to the type genome, which has been assigned the MAG ID ChiSxjej1B13-7958 and which is available via NCBI BioSample SAMN15817070. The GC content of the type genome is $58.28 \%$ and the genome length is 2.2 Mbp.

\section{Description of Candidatus Caccousia avistercoris sp. nov.}

Candidatus Caccousia avistercoris (a.vi.ster'co.ris. L. fem. n. avis bird; L. neut. n. stercus dung; N.L. gen. n. avistercoris of bird faeces)

A bacterial species identified by metagenomic analyses. This species includes all bacteria with genomes that show $\geq 95 \%$ average nucleotide identity (ANI) to the type genome, which has been assigned the MAG ID 3024 and which is available via NCBI BioSample SAMN15817047. The $\mathrm{GC}$ content of the type genome is $60.88 \%$ and the genome length is $2.6 \mathrm{Mbp}$.

\section{Description of Candidatus Caccousia stercoris sp. nov. \\ Candidatus Caccousia stercoris (ster'co.ris. L. gen. n. stercoris of dung, excrement)}

A bacterial species identified by metagenomic analyses. This species includes all bacteria with genomes that show $\geq 95 \%$ average nucleotide identity (ANI) to the type genome, which has been assigned the MAG ID 6086 and which is available via NCBI BioSample SAMN15817184. This

Peer] reviewing PDF | (2020:12:55980:1:1:NEW 13 Jan 2021) 
2287 is a new name for the alphanumeric GTDB species sp002160025. The GC content of the type

2288

2289

2290

2291

2292

2293

2294

2295

2296

2297

2298

2299

2300

2301

2302

2303

2304

2305

2306

2307

2308

2309

2310

2311

2312

2313

2314

2315

2316

2317

2318

2319

2320

2321

2322

2323

2324

2325

2326

2327

2328

2329

2330

2331

2332

genome is $56.84 \%$ and the genome length is $2.3 \mathrm{Mbp}$.

\section{Description of Candidatus Caccovicinus gen. nov.}

Candidatus Caccovicinus (Cacc.o.vic.in'us. Gr. fem. n. kakke dung; L. masc. n. vicinus a neighbour; N.L. masc. n. Caccovicinus a microbe associated with faeces)

A bacterial genus identified by metagenomic analyses. The genus includes all bacteria with genomes that show $\geq 60 \%$ average amino acid identity (AAI) to the type genome from the type species Caccovicinus merdipullorum. This is a name for the alphanumeric GTDB genus UMGS1370. This genus has been assigned by GTDB-Tk v1.3.0 working on GTDB Release 05RS95 (Chaumeil et al., 2019; Parks et al., 2020) to the order Lachnospirales and to the family Lachnospiraceae.

\section{Description of Candidatus Caccovicinus merdipullorum sp. nov.}

Candidatus Caccovicinus merdipullorum (mer.di.pul.lo'rum. L. fem. n. merda faeces; L. masc. n. pullus a young chicken; N.L. gen. n. merdipullorum of the faeces of young chickens)

A bacterial species identified by metagenomic analyses. This species includes all bacteria with genomes that show $\geq 95 \%$ average nucleotide identity (ANI) to the type genome, which has been assigned the MAG ID CHK198_11255 and which is available via NCBI BioSample SAMN15817041. The GC content of the type genome is $50.44 \%$ and the genome length is 3.0 Mbp.

\section{Description of Candidatus Caccovivens gen. nov.}

Candidatus Caccovivens (Cacc.o.viv'ens. Gr. fem. n. kakke dung; N.L. pres. part. vivens living; N.L. fem. n. Caccovivens a microbe associated with faeces)

A bacterial genus identified by metagenomic analyses. The genus includes all bacteria with genomes that show $\geq 60 \%$ average amino acid identity (AAI) to the type genome from the type species Caccovivens faecavium. This is a name for the alphanumeric GTDB genus UBA11517. This genus has been assigned by GTDB-Tk v1.3.0 working on GTDB Release 05-RS95 (Chaumeil et al., 2019; Parks et al., 2020) to the order Christensenellales and to the family UBA1242.

\section{Description of Candidatus Caccovivens faecavium sp. nov.}

Candidatus Caccovivens faecavium (faec.a'vi.um. L. fem. n. faex, faecis excrement; L. fem. n. avis bird; N.L. gen. n. faecavium of bird faeces)

A bacterial species identified by metagenomic analyses. This species includes all bacteria with genomes that show $\geq 95 \%$ average nucleotide identity (ANI) to the type genome, which has been assigned the MAG ID ChiW6-1002 and which is available via NCBI BioSample

Peer] reviewing PDF | (2020:12:55980:1:1:NEW 13 Jan 2021) 
2333 SAMN15817119. The GC content of the type genome is $34.22 \%$ and the genome length is 1.1

2334 Mbp.

2335

2336

2337

2338

2339

2340

2341

2342

2343

2344

2345

2346

2347

2348

2349

2350

2351

2352

2353

2354

2355

2356

2357

2358

2359

Description of Candidatus Cellulosilyticum pullistercoris sp. nov.

Candidatus Cellulosilyticum pullistercoris (pul.li.ster'co.ris. L. masc. n. pullus a young chicken; L. neut. n. stercus dung; N.L. gen. n. pullistercoris of young chicken faeces)

A bacterial species identified by metagenomic analyses. This species includes all bacteria with genomes that show $\geq 95 \%$ average nucleotide identity (ANI) to the type genome, which has been assigned the MAG ID B5-657 and which is available via NCBI BioSample SAMN15816752. The GC content of the type genome is $33.78 \%$ and the genome length is $2.3 \mathrm{Mbp}$.

\section{Description of Candidatus Choladocola gen. nov.}

Candidatus Choladocola (Cho.lad.o.co'la. Gr. fem. n. cholas guts; L. suff. -cola inhabitant of; N.L. fem. n. Choladocola a microbe associated with the intestines)

A bacterial genus identified by metagenomic analyses. The genus includes all bacteria with genomes that show $\geq 60 \%$ average amino acid identity (AAI) to the type genome from the type species Choladocola avistercoris. This is a name for the alphanumeric GTDB genus UBA7182. This genus has been assigned by GTDB-Tk v1.3.0 working on GTDB Release 05-RS95 (Chaumeil et al., 2019; Parks et al., 2020) to the order Lachnospirales and to the family Lachnospiraceae.

\section{Description of Candidatus Choladocola avistercoris sp. nov.}

2360 Candidatus Choladocola avistercoris (a.vi.ster'co.ris. L. fem. n. avis bird; L. neut. n. stercus

2361

2362

2363 dung; N.L. gen. n. avistercoris of bird faeces)

2364

2365

2366

2367

2368

2369

2370

2371

2372

2373

2374

2375

2376

2377

A bacterial species identified by metagenomic analyses. This species includes all bacteria with genomes that show $\geq 95 \%$ average nucleotide identity (ANI) to the type genome, which has been assigned the MAG ID ChiBcec18-1958 and which is available via NCBI BioSample SAMN15817180. This is a new name for the alphanumeric GTDB species sp002160135. The $\mathrm{GC}$ content of the type genome is $50.79 \%$ and the genome length is $2.4 \mathrm{Mbp}$.

\section{Description of Candidatus Choladousia gen. nov.}

Candidatus Choladousia (Cho.lad.ou'si.a. Gr. fem. n. cholas guts; Gr. fem. n. ousia an essence; N.L. fem. n. Choladousia a microbe associated with the intestines)

A bacterial genus identified by metagenomic analyses. The genus includes all bacteria with genomes that show $\geq 60 \%$ average amino acid identity (AAI) to the type genome from the type species Choladousia intestinavium. This is a name for the alphanumeric GTDB genus UBA7160. This genus has been assigned by GTDB-Tk v1.3.0 working on GTDB Release 05-RS95 
2378 (Chaumeil et al., 2019; Parks et al., 2020) to the order Lachnospirales and to the family

2379 Lachnospiraceae.

2380

2381

2382

2383

2384

2385

2386

2387

2388

2389

2390

2391

2392

2393

2394

2395

2396

2397

2398

2399

2400

2401

2402

2403

2404

2405

2406

2407

2408

2409

2410

2411

2412

2413

2414

2415

2416

2417

2418

2419

2420

2421

2422

2423

\author{
Description of Candidatus Choladousia intestinavium sp. nov. \\ Candidatus Choladousia intestinavium (in.tes.tin.a'vi.um. L. neut. n. intestinum gut; L. fem. n. \\ avis bird; N.L. gen. n. intestinavium of the gut of birds) \\ A bacterial species identified by metagenomic analyses. This species includes all bacteria with \\ genomes that show $\geq 95 \%$ average nucleotide identity (ANI) to the type genome, which has been \\ assigned the MAG ID ChiSjej4B22-8148 and which is available via NCBI BioSample \\ SAMN15817012. The GC content of the type genome is $49.57 \%$ and the genome length is 2.9 \\ Mbp.
}

\title{
Description of Candidatus Choladousia intestinigallinarum sp. nov. Candidatus Choladousia intestinigallinarum (in.tes.ti.ni.gal.li.na'rum. L. neut. n. intestinum gut; L. fem. n. gallina hen; N.L. gen. n. intestinigallinarum of the gut of the hens)
}

A bacterial species identified by metagenomic analyses. This species includes all bacteria with genomes that show $\geq 95 \%$ average nucleotide identity (ANI) to the type genome, which has been assigned the MAG ID ChiBcec11-13528 and which is available via NCBI BioSample SAMN15817065. The GC content of the type genome is $48.69 \%$ and the genome length is 3.1 Mbp.

\section{Description of Candidatus Choladousia intestinipullorum sp. nov.}

Candidatus Choladousia intestinipullorum (in.tes.ti.ni.pul.lo'rum. L. neut. n. intestinum gut; L. masc. n. pullus a young chicken; N.L. gen. n. intestinipullorum of the gut of young chickens)

A bacterial species identified by metagenomic analyses. This species includes all bacteria with genomes that show $\geq 95 \%$ average nucleotide identity (ANI) to the type genome, which has been assigned the MAG ID ChiSjej5B23-16397 and which is available via NCBI BioSample SAMN15817078. The GC content of the type genome is $50.54 \%$ and the genome length is 2.2 Mbp.

\section{Description of Candidatus Collinsella stercoripullorum sp. nov.}

Candidatus Collinsella stercoripullorum (ster.co.ri.pul.lo'rum. L. neut. n. stercus dung; L. masc. n. pullus a young chicken; N.L. gen. n. stercoripullorum of the faceces of young chickens)

A bacterial species identified by metagenomic analyses. This species includes all bacteria with genomes that show $\geq 95 \%$ average nucleotide identity (ANI) to the type genome, which has been assigned the MAG ID ChiGjej6B6-20822 and which is available via NCBI BioSample SAMN15816681. The GC content of the type genome is $68.61 \%$ and the genome length is 2.3 Mbp. 
Description of Candidatus Companilactobacillus pullicola sp. nov.

Candidatus Companilactobacillus pullicola (pul.li'co.la. L. masc. n. pullus a young chicken; L. suff. -cola inhabitant of; N.L. n. pullicola an inhabitant of young chickens)

2429

2430

A bacterial species identified by metagenomic analyses. This species includes all bacteria with genomes that show $\geq 95 \%$ average nucleotide identity (ANI) to the type genome, which has been assigned the MAG ID 3204 and which is available via NCBI BioSample SAMN15816700. The $\mathrm{GC}$ content of the type genome is $35.87 \%$ and the genome length is $2.9 \mathrm{Mbp}$.

2433

2434

2435

2436

2437

Description of Candidatus Coprenecus gen. nov.

Candidatus Coprenecus (Copr.en.e'cus. Gr. fem. n. kopros dung; Gr. masc. enoikos inhabitant;

2438

2439

2440

N.L. masc. n. Coprenecus a microbe associated with faeces)

A bacterial genus identified by metagenomic analyses. The genus includes all bacteria with genomes that show $\geq 60 \%$ average amino acid identity (AAI) to the type genome from the type species Coprenecus pullicola. This is a name for the alphanumeric GTDB genus CAG-831. This genus has been assigned by GTDB-Tk v1.3.0 working on GTDB Release 05-RS95 (Chaumeil et al., 2019; Parks et al., 2020) to the order Bacteroidales and to the family UBA932.

2445

2446

2447

2448

2449

2450

2451

2452

2453

2454

2455

2456

2457

2458

2459

Description of Candidatus Coprenecus avistercoris sp. nov.

Candidatus Coprenecus avistercoris (a.vi.ster'co.ris. L. fem. n. avis bird; L. neut. n. stercus dung; N.L. gen. n. avistercoris of bird faeces)

A bacterial species identified by metagenomic analyses. This species includes all bacteria with genomes that show $\geq 95 \%$ average nucleotide identity (ANI) to the type genome, which has been assigned the MAG ID ChiHjej13B12-12457 and which is available via NCBI BioSample SAMN15817175. This is a new name for the alphanumeric GTDB species sp000432775. The $\mathrm{GC}$ content of the type genome is $56.44 \%$ and the genome length is $2.0 \mathrm{Mbp}$.

\section{Description of Candidatus Coprenecus merdigallinarum sp. nov.}

Candidatus Coprenecus merdigallinarum (mer.di.gal.li.na'rum. L. fem. n. merda faeces; L. fem.

2460

2461

2462

2463

2464

2465

2466

2467

n. gallina hen; N.L. gen. n. merdigallinarum of hen faeces)

A bacterial species identified by metagenomic analyses. This species includes all bacteria with genomes that show $\geq 95 \%$ average nucleotide identity (ANI) to the type genome, which has been assigned the MAG ID ChiHecolR1B25-18470 and which is available via NCBI BioSample SAMN15817066. The GC content of the type genome is $55.94 \%$ and the genome length is 2.2 Mbp. 
2470 Candidatus Coprenecus merdipullorum (mer.di.pul.lo'rum. L. fem. n. merda faeces; L. masc. $\mathrm{n}$.

2471

2472

2473

2474

2475

2476

2477

2478

2479

2480

2481

2482

2483

2484

2485

2486

2487

2488

2489

2490

2491

2492

2493

2494

2495

2496

2497

2498

2499

2500

2501

2502

2503

2504

2505

2506

2507

2508

2509

2510

2511

2512

2513

2514

2515

pullus a young chicken; N.L. gen. n. merdipullorum of the faeces of young chickens)

A bacterial species identified by metagenomic analyses. This species includes all bacteria with genomes that show $\geq 95 \%$ average nucleotide identity (ANI) to the type genome, which has been assigned the MAG ID Gambia1 1-1358 and which is available via NCBI BioSample SAMN15817068. The GC content of the type genome is $54.29 \%$ and the genome length is 2.1 Mbp.

\section{Description of Candidatus Coprenecus pullicola sp. nov.}

Candidatus Coprenecus pullicola (pul.li'co.la. L. masc. n. pullus a young chicken; L. suff. -cola inhabitant of; N.L. n. pullicola an inhabitant of young chickens)

A bacterial species identified by metagenomic analyses. This species includes all bacteria with genomes that show $\geq 95 \%$ average nucleotide identity (ANI) to the type genome, which has been assigned the MAG ID ChiHjej9B8-15444 and which is available via NCBI BioSample SAMN15817077. The GC content of the type genome is $52.53 \%$ and the genome length is 2.1 Mbp.

\section{Description of Candidatus Coprenecus pullistercoris sp. nov.}

Candidatus Coprenecus pullistercoris (pul.li.ster'co.ris. L. masc. n. pullus a young chicken; L. neut. n. stercus dung; N.L. gen. n. pullistercoris of young chicken faeces)

A bacterial species identified by metagenomic analyses. This species includes all bacteria with genomes that show $\geq 95 \%$ average nucleotide identity (ANI) to the type genome, which has been assigned the MAG ID Gambia18-42 and which is available via NCBI BioSample

SAMN15817084. The GC content of the type genome is $52.64 \%$ and the genome length is 2.0 Mbp.

\section{Description of Candidatus Coprenecus stercoravium sp. nov.}

Candidatus Coprenecus stercoravium (ster.cor.a'vi.um. L. neut. n. stercus dung; L. fem. n. avis bird; N.L. gen. n. stercoravium of bird faeces)

A bacterial species identified by metagenomic analyses. This species includes all bacteria with genomes that show $\geq 95 \%$ average nucleotide identity (ANI) to the type genome, which has been assigned the MAG ID Gambia16-554 and which is available via NCBI BioSample SAMN15817085. The GC content of the type genome is $51.84 \%$ and the genome length is 1.8 Mbp.

\section{Description of Candidatus Coprenecus stercorigallinarum sp. nov.} Candidatus Coprenecus stercorigallinarum (ster.co.ri.gal.li.na'rum. L. neut. n. stercus dung; L. fem. n. gallina hen; N.L. gen. n. stercorigallinarum of hen faeces) 
2516

2517

2518

2519

2520

2521

2522

2523

2524

2525

2526

2527

2528

2529

2530

2531

2532

2533

2534

2535

2536

2537

2538

2539

2540

2541

2542

2543

2544

2545

2546

2547

2548

2549

2550

2551

2552

2553

2554

2555

2556

2557

2558

2559

2560

2561

A bacterial species identified by metagenomic analyses. This species includes all bacteria with genomes that show $\geq 95 \%$ average nucleotide identity (ANI) to the type genome, which has been assigned the MAG ID 3382 and which is available via NCBI BioSample SAMN15817086. The GC content of the type genome is $53.66 \%$ and the genome length is $2.1 \mathrm{Mbp}$.

\section{Description of Candidatus Coprenecus stercoripullorum sp. nov.}

Candidatus Coprenecus stercoripullorum (ster.co.ri.pul.lo'rum. L. neut. n. stercus dung; L. masc. n. pullus a young chicken; N.L. gen. n. stercoripullorum of the faceces of young chickens)

A bacterial species identified by metagenomic analyses. This species includes all bacteria with genomes that show $\geq 95 \%$ average nucleotide identity (ANI) to the type genome, which has been assigned the MAG ID 7141 and which is available via NCBI BioSample SAMN15817115. The $\mathrm{GC}$ content of the type genome is $50.92 \%$ and the genome length is $1.7 \mathrm{Mbp}$.

\section{Description of Candidatus Coprocola gen. nov.}

Candidatus Coprocola (Copr.o.co'la. Gr. fem. n. kopros dung; L. suff. -cola inhabitant of; N.L. fem. n. Coprocola a microbe associated with faeces)

A bacterial genus identified by metagenomic analyses. The genus includes all bacteria with genomes that show $\geq 60 \%$ average amino acid identity (AAI) to the type genome from the type species Coprocola pullicola. This is a name for the alphanumeric GTDB genus ASF356. This genus has been assigned by GTDB-Tk v1.3.0 working on GTDB Release 05-RS95 (Chaumeil et al., 2019; Parks et al., 2020) to the order Lachnospirales and to the family Anaerotignaceae.

\section{Description of Candidatus Coprocola pullicola sp. nov.}

Candidatus Coprocola pullicola (pul.li'co.la. L. masc. n. pullus a young chicken; L. suff. -cola inhabitant of; N.L. n. pullicola an inhabitant of young chickens)

A bacterial species identified by metagenomic analyses. This species includes all bacteria with genomes that show $\geq 95 \%$ average nucleotide identity (ANI) to the type genome, which has been assigned the MAG ID CHK193-15662 and which is available via NCBI BioSample SAMN15817043. The GC content of the type genome is $33.91 \%$ and the genome length is 3.0 Mbp.

\section{Description of Candidatus Copromonas gen. nov.}

Candidatus Copromonas (Copr.omo'nas. Gr. fem. n. kopros dung; L. fem. n. monas a monad; N.L. fem. n. Copromonas a microbe associated with faeces)

A bacterial genus identified by metagenomic analyses. The genus includes all bacteria with genomes that show $\geq 60 \%$ average amino acid identity (AAI) to the type genome from the type species Copromonas avistercoris. This is a name for the alphanumeric GTDB genus CAG- 81 .

PeerJ reviewing PDF | (2020:12:55980:1:1:NEW 13 Jan 2021) 
2562 This genus has been assigned by GTDB-Tk v1.3.0 working on GTDB Release 05-RS95

2563 (Chaumeil et al., 2019; Parks et al., 2020) to the order Lachnospirales and to the family

2564 Lachnospiraceae.

2565

2566

2567

2568

2569

2570

2571

2572

2573

2574

2575

2576

2577

2578

2579

2580

2581

2582

2583

2584

2585

2586

2587

2588

2589

2590

2591

2592

2593

2594

2595

2596

2597

2598

2599

2600

2601

2602

2603

2604

2605

2606

2607

\begin{abstract}
Description of Candidatus Copromonas avistercoris sp. nov.
Candidatus Copromonas avistercoris (a.vi.ster'co.ris. L. fem. n. avis bird; L. neut. n. stercus dung; N.L. gen. n. avistercoris of bird faeces)
\end{abstract}

A bacterial species identified by metagenomic analyses. This species includes all bacteria with genomes that show $\geq 95 \%$ average nucleotide identity (ANI) to the type genome, which has been assigned the MAG ID ChiSjej3B21-5768 and which is available via NCBI BioSample SAMN15817005. The GC content of the type genome is $52.88 \%$ and the genome length is 2.2 Mbp.

\title{
Description of Candidatus Copromonas faecavium sp. nov.
}

Candidatus Copromonas faecavium (faec.a'vi.um. L. fem. n. faex, faecis excrement; L. fem. n. avis bird; N.L. gen. n. faecavium of bird faeces)

A bacterial species identified by metagenomic analyses. This species includes all bacteria with genomes that show $\geq 95 \%$ average nucleotide identity (ANI) to the type genome, which has been assigned the MAG ID CHK180-2868 and which is available via NCBI BioSample SAMN15817009. The GC content of the type genome is $50.20 \%$ and the genome length is 2.7 Mbp.

\section{Description of Candidatus Copromorpha gen. nov.}

Candidatus Copromorpha (Copr.o.mor'pha. Gr. fem. n. kopros dung; Gr. fem. n. morphe a form, shape; N.L. fem. n. Copromorpha a microbe associated with faeces)

A bacterial genus identified by metagenomic analyses. The genus includes all bacteria with genomes that show $\geq 60 \%$ average amino acid identity (AAI) to the type genome from the type species Copromorpha excrementavium. This is a name for the alphanumeric GTDB genus UBA1191. This genus has been assigned by GTDB-Tk v1.3.0 working on GTDB Release 05RS95 (Chaumeil et al., 2019; Parks et al., 2020) to the order Peptostreptococcales and to the family Anaerovoracaceae.

\section{Description of Candidatus Copromorpha excrementavium sp. nov.}

Candidatus Copromorpha excrementavium (ex.cre.ment.a'vi.um. L. neut. n. excrementum excrement; L. fem. n. avis bird; N.L. gen. n. excrementavium of bird excrement)

A bacterial species identified by metagenomic analyses. This species includes all bacteria with genomes that show $\geq 95 \%$ average nucleotide identity (ANI) to the type genome, which has been assigned the MAG ID CHK176-22527 and which is available via NCBI BioSample 
2608 SAMN15817193. This is a new name for the alphanumeric GTDB species sp900542385. The

2609

2610

2611

2612

2613

2614

2615

2616

2617

2618

2619

2620

2621

2622

2623

2624

2625

2626

2627

2628

2629

2630

2631

2632

2633

2634

2635

2636

2637

2638

2639

2640

2641

2642

2643

2644

2645

2646

2647

2648

2649

2650

2651

2652

2653

$\mathrm{GC}$ content of the type genome is $42.88 \%$ and the genome length is $1.8 \mathrm{Mbp}$.

\section{Description of Candidatus Copromorpha excrementigallinarum sp. nov.}

Candidatus Copromorpha excrementigallinarum (ex.cre.men.ti.gal.li.na'rum. L. neut. n. excrementum excrement; L. fem. n. gallina hen; N.L. gen. n. excrementigallinarum of hen excrement)

A bacterial species identified by metagenomic analyses. This species includes all bacteria with genomes that show $\geq 95 \%$ average nucleotide identity (ANI) to the type genome, which has been assigned the MAG ID ChiHcec3-6078 and which is available via NCBI BioSample SAMN15817131. The GC content of the type genome is $48.15 \%$ and the genome length is 2.0 Mbp.

\section{Description of Candidatus Copromorpha excrementipullorum sp. nov.} Candidatus Copromorpha excrementipullorum (ex.cre.men.ti.pul.lo'rum. L. neut. n. excrementum excrement; L. masc. n. pullus a young chicken; N.L. gen. n. excrementipullorum of young chicken excrement)

A bacterial species identified by metagenomic analyses. This species includes all bacteria with genomes that show $\geq 95 \%$ average nucleotide identity (ANI) to the type genome, which has been assigned the MAG ID ChiSjej4B22-8349 and which is available via NCBI BioSample SAMN15817205. This is a new name for the alphanumeric GTDB species sp900543485. The $\mathrm{GC}$ content of the type genome is $49.77 \%$ and the genome length is $1.9 \mathrm{Mbp}$.

\section{Description of Candidatus Coproplasma gen. nov.}

Candidatus Coproplasma (Copr.o.plas'ma. Gr. fem. n. kopros dung; Gr. neut. n. plasma a form; N.L. neut. n. Coproplasma a microbe associated with faeces)

A bacterial genus identified by metagenomic analyses. The genus includes all bacteria with genomes that show $\geq 60 \%$ average amino acid identity (AAI) to the type genome from the type species Coproplasma stercoravium. This is a name for the alphanumeric GTDB genus UBA11940. This genus has been assigned by GTDB-Tk v1.3.0 working on GTDB Release 05RS95 (Chaumeil et al., 2019; Parks et al., 2020) to the order Christensenellales and to the family Borkfalkiaceae.

\section{Description of Candidatus Coproplasma avicola sp. nov.}

Candidatus Coproplasma avicola (a.vi'co.la. L. fem. n. avis bird; L. suff. -cola inhabitant of; N.L. n. avicola inhabitant of birds)

A bacterial species identified by metagenomic analyses. This species includes all bacteria with genomes that show $\geq 95 \%$ average nucleotide identity (ANI) to the type genome, which has been 
2654 assigned the MAG ID ChiW16-3235 and which is available via NCBI BioSample

2655 SAMN15817075. The GC content of the type genome is $51.11 \%$ and the genome length is 1.5

$2656 \mathrm{Mbp}$.

2657

2658

2659

Description of Candidatus Coproplasma avistercoris sp. nov.

2660 Candidatus Coproplasma avistercoris (a.vi.ster'co.ris. L. fem. n. avis bird; L. neut. n. stercus

2661

2662

2663

dung; N.L. gen. n. avistercoris of bird faeces)

2664

A bacterial species identified by metagenomic analyses. This species includes all bacteria with genomes that show $\geq 95 \%$ average nucleotide identity (ANI) to the type genome, which has been

2665 assigned the MAG ID ChiW7-3743 and which is available via NCBI BioSample

2666

2667

SAMN15817023. The GC content of the type genome is $56.32 \%$ and the genome length is 1.4 Mbp.

Description of Candidatus Coproplasma excrementavium sp. nov.

2671

Candidatus Coproplasma excrementavium (ex.cre.ment.a'vi.um. L. neut. n. excrementum

2672

2673

2674

excrement; L. fem. n. avis bird; N.L. gen. n. excrementavium of bird excrement)

A bacterial species identified by metagenomic analyses. This species includes all bacteria with

2675

2676

2677

2678

2679 genomes that show $\geq 95 \%$ average nucleotide identity (ANI) to the type genome, which has been assigned the MAG ID CHK179-18245 and which is available via NCBI BioSample SAMN15817045. The GC content of the type genome is $50.26 \%$ and the genome length is 1.4 Mbp.

2680

2681

Description of Candidatus Coproplasma excrementigallinarum sp. nov.

2682

Candidatus Coproplasma excrementigallinarum (ex.cre.men.ti.gal.li.na'rum. L. neut. $n$.

2683

excrementum excrement; L. fem. n. gallina hen; N.L. gen. n. excrementigallinarum of hen

2684

excrement)

2685

2686

2687

2688

A bacterial species identified by metagenomic analyses. This species includes all bacteria with genomes that show $\geq 95 \%$ average nucleotide identity (ANI) to the type genome, which has been assigned the MAG ID CHK195-12923 and which is available via NCBI BioSample

2689

2690

2691

2692

2693

SAMN15817050. The GC content of the type genome is $50.00 \%$ and the genome length is 1.4 Mbp.

2694

Description of Candidatus Coproplasma excrementipullorum sp. nov.

Candidatus Coproplasma excrementipullorum (ex.cre.men.ti.pul.lo'rum. L. neut. n. excrementum

2695

2696

2697

2698

excrement; L. masc. n. pullus a young chicken; N.L. gen. n. excrementipullorum of young chicken excrement)

2699

A bacterial species identified by metagenomic analyses. This species includes all bacteria with genomes that show $\geq 95 \%$ average nucleotide identity (ANI) to the type genome, which has been 
2700 assigned the MAG ID 10570 and which is available via NCBI BioSample SAMN15817148. The

2701

2702

2703

2704

2705

2706

2707

2708

2709

2710

2711

2712

2713

2714

2715

2716

2717

2718

2719

2720

2721

2722

2723

2724

2725

2726

2727

2728

2729

2730

2731

2732

2733

2734

2735

2736

2737

2738

2739

2740

2741

2742

2743

2744

2745

$\mathrm{GC}$ content of the type genome is $51.24 \%$ and the genome length is $1.7 \mathrm{Mbp}$.

\section{Description of Candidatus Coproplasma stercoravium sp. nov.}

Candidatus Coproplasma stercoravium (ster.cor.a'vi.um. L. neut. n. stercus dung; L. fem. n. avis bird; N.L. gen. n. stercoravium of bird faeces)

A bacterial species identified by metagenomic analyses. This species includes all bacteria with genomes that show $\geq 95 \%$ average nucleotide identity (ANI) to the type genome, which has been assigned the MAG ID CHK180-19203 and which is available via NCBI BioSample SAMN15817006. The GC content of the type genome is $51.86 \%$ and the genome length is 1.6 Mbp.

\section{Description of Candidatus Coproplasma stercorigallinarum sp. nov.}

Candidatus Coproplasma stercorigallinarum (ster.co.ri.gal.li.na'rum. L. neut. n. stercus dung; L. fem. n. gallina hen; N.L. gen. n. stercorigallinarum of hen faeces)

A bacterial species identified by metagenomic analyses. This species includes all bacteria with genomes that show $\geq 95 \%$ average nucleotide identity (ANI) to the type genome, which has been assigned the MAG ID CHK191-24566 and which is available via NCBI BioSample SAMN15817171. This is a new name for the alphanumeric GTDB species sp900549005. The $\mathrm{GC}$ content of the type genome is $51.83 \%$ and the genome length is $1.6 \mathrm{Mbp}$.

\section{Description of Candidatus Coproplasma stercoripullorum sp. nov.}

Candidatus Coproplasma stercoripullorum (ster.co.ri.pul.lo'rum. L. neut. n. stercus dung; L. masc. n. pullus a young chicken; N.L. gen. n. stercoripullorum of the faceces of young chickens)

A bacterial species identified by metagenomic analyses. This species includes all bacteria with genomes that show $\geq 95 \%$ average nucleotide identity (ANI) to the type genome, which has been assigned the MAG ID ChiW25-3613 and which is available via NCBI BioSample SAMN15817071. The GC content of the type genome is $51.17 \%$ and the genome length is 1.7 Mbp.

\section{Description of Candidatus Coprosoma gen. nov.}

Candidatus Coprosoma (Copr.o.so'ma. Gr. fem. n. kopros dung; Gr. neut. n. soma a body; N.L. neut. n. Coprosoma a microbe associated with faeces)

A bacterial genus identified by metagenomic analyses. The genus includes all bacteria with genomes that show $\geq 60 \%$ average amino acid identity (AAI) to the type genome from the type species Coprosoma intestinipullorum. This is a name for the alphanumeric GTDB genus CAG822. This genus has been assigned by GTDB-Tk v1.3.0 working on GTDB Release 05-RS95 (Chaumeil et al., 2019; Parks et al., 2020) to the order RF39 and to the family CAG-822.

Peer) reviewing PDF | (2020:12:55980:1:1:NEW 13 Jan 2021) 
2746

2747

2748

2749

2750

2751

2752

2753

2754

2755

2756

2757

2758

2759

2760

2761

2762

2763

2764

2765

2766

2767

2768

2769

2770

2771

2772

2773

2774

2775

2776

2777

2778

2779

2780

2781

2782

2783

2784

2785

2786

2787

2788

2789

2790

2791

\section{Description of Candidatus Coprosoma intestinipullorum sp. nov.}

Candidatus Coprosoma intestinipullorum (in.tes.ti.ni.pul.lo'rum. L. neut. n. intestinum gut; L. masc. n. pullus a young chicken; N.L. gen. n. intestinipullorum of the gut of young chickens)

A bacterial species identified by metagenomic analyses. This species includes all bacteria with genomes that show $\geq 95 \%$ average nucleotide identity (ANI) to the type genome, which has been assigned the MAG ID CHK147-3167 and which is available via NCBI BioSample

SAMN15817008. The GC content of the type genome is $31.20 \%$ and the genome length is 1.3 Mbp.

\section{Description of Candidatus Coprousia gen. nov.}

Candidatus Coprousia (Copr.ou'si.a. Gr. fem. n. kopros dung; Gr. fem. n. ousia an essence; N.L. fem. n. Coprousia a microbe associated with faeces)

A bacterial genus identified by metagenomic analyses. The genus includes all bacteria with genomes that show $\geq 60 \%$ average amino acid identity (AAI) to the type genome from the type species Coprousia avicola. This is a name for the alphanumeric GTDB genus An7. This genus has been assigned by GTDB-Tk v1.3.0 working on GTDB Release 05-RS95 (Chaumeil et al., 2019; Parks et al., 2020) to the order Coriobacteriales and to the family Coriobacteriaceae.

\section{Description of Candidatus Coprousia avicola sp. nov.}

Candidatus Coprousia avicola (a.vi'co.la. L. fem. n. avis bird; L. suff. -cola inhabitant of; N.L. n. avicola inhabitant of birds)

A bacterial species identified by metagenomic analyses. This species includes all bacteria with genomes that show $\geq 95 \%$ average nucleotide identity (ANI) to the type genome, which has been assigned the MAG ID ChiHjej11B10-5566 and which is available via NCBI BioSample SAMN15817195. This is a new name for the alphanumeric GTDB species sp002159765. The $\mathrm{GC}$ content of the type genome is $65.47 \%$ and the genome length is $2.4 \mathrm{Mbp}$.

\section{Description of Candidatus Coprovicinus gen. nov.}

Candidatus Coprovicinus (Copr.o.vic.in'us. Gr. fem. n. kopros dung; L. masc. n. vicinus a neighbour; N.L. masc. n. Coprovicinus a microbe associated with faeces)

A bacterial genus identified by metagenomic analyses. The genus includes all bacteria with genomes that show $\geq 60 \%$ average amino acid identity (AAI) to the type genome from the type species Coprovicinus avistercoris. This is a name for the alphanumeric GTDB genus UMGS1418. This genus has been assigned by GTDB-Tk v1.3.0 working on GTDB Release 05RS95 (Chaumeil et al., 2019; Parks et al., 2020) to the order Coriobacteriales and to the family Atopobiaceae.

PeerJ reviewing PDF | (2020:12:55980:1:1:NEW 13 Jan 2021) 
Description of Candidatus Coprovicinus avistercoris sp. nov.

2794

2795

Candidatus Coprovicinus avistercoris (a.vi.ster'co.ris. L. fem. n. avis bird; L. neut. n. stercus dung; N.L. gen. n. avistercoris of bird faeces)

2796

2797

A bacterial species identified by metagenomic analyses. This species includes all bacteria with

2798

2799 genomes that show $\geq 95 \%$ average nucleotide identity (ANI) to the type genome, which has been assigned the MAG ID ChiHjej12B11-29160 and which is available via NCBI BioSample

2800 SAMN15817198. This is a new name for the alphanumeric GTDB species sp900551595. The

2801 $\mathrm{GC}$ content of the type genome is $49.51 \%$ and the genome length is $1.7 \mathrm{Mbp}$.

2802

2803

2804

2805

Description of Candidatus Coprovivens gen. nov.

Candidatus Coprovivens (Copr.o.viv'ens. Gr. fem. n. kopros dung; N.L. pres. part. vivens living;

2806

2807

2808 N.L. fem. n. Coprovivens a microbe associated with faeces)

2809

2810

A bacterial genus identified by metagenomic analyses. The genus includes all bacteria with genomes that show $\geq 60 \%$ average amino acid identity (AAI) to the type genome from the type species Coprovivens excrementavium. This is a name for the alphanumeric GTDB genus

2811 UBA11963. This genus has been assigned by GTDB-Tk v1.3.0 working on GTDB Release 05-

2812 RS95 (Chaumeil et al., 2019; Parks et al., 2020) to the order RF39 and to the family $C A G-1000$.

2813

2814

2815

Description of Candidatus Coprovivens excrementavium sp. nov.

2816

2817

Candidatus Coprovivens excrementavium (ex.cre.ment.a'vi.um. L. neut. n. excrementum excrement; L. fem. n. avis bird; N.L. gen. n. excrementavium of bird excrement)

2818

2819

2820

2821

2822

2823

2824

2825

2826

2827

2828

2829

2830

2831

2832

2833

2834

2835

2836

A bacterial species identified by metagenomic analyses. This species includes all bacteria with genomes that show $\geq 95 \%$ average nucleotide identity (ANI) to the type genome, which has been assigned the MAG ID 3297 and which is available via NCBI BioSample SAMN15817083. The $\mathrm{GC}$ content of the type genome is $28.25 \%$ and the genome length is $2.2 \mathrm{Mbp}$.

\section{Description of Candidatus Corynebacterium avicola sp. nov.}

Candidatus Corynebacterium avicola (a.vi'co.la. L. fem. n. avis bird; L. suff. -cola inhabitant of; N.L. n. avicola inhabitant of birds)

A bacterial species identified by metagenomic analyses. This species includes all bacteria with genomes that show $\geq 95 \%$ average nucleotide identity (ANI) to the type genome, which has been assigned the MAG ID CHK32-1732 and which is available via NCBI BioSample SAMN15816750. The GC content of the type genome is $66.95 \%$ and the genome length is 3.1 Mbp.

\section{Description of Candidatus Corynebacterium faecigallinarum sp. nov.}


2837 Candidatus Corynebacterium faecigallinarum (fae.ci.gal.li.na'rum. L. fem. n. faex, faecis

2838

2839

2840

2841

2842

2843

2844

2845

2846

2847

2848

2849

2850

2851

2852

2853

2854

2855

2856

2857

2858

2859

2860

2861

2862

2863

2864

2865

2866

2867

2868

2869

2870

2871

2872

2873

2874

2875

2876

2877

2878

2879

2880

2881

2882

excrement; L. fem. n. gallina hen; N.L. gen. n. faecigallinarum of hen faeces)

A bacterial species identified by metagenomic analyses. This species includes all bacteria with genomes that show $\geq 95 \%$ average nucleotide identity (ANI) to the type genome, which has been assigned the MAG ID ChiHjej13B12-4958 and which is available via NCBI BioSample SAMN15816631. The GC content of the type genome is $66.91 \%$ and the genome length is 2.8 Mbp.

\section{Description of Candidatus Corynebacterium faecipullorum sp. nov.}

Candidatus Corynebacterium faecipullorum (fae.ci.pul.lo'rum. L. fem. n. faex, faecis excrement; L. masc. n. pullus a young chicken; N.L. gen. n. faecipullorum of young chicken faeces)

A bacterial species identified by metagenomic analyses. This species includes all bacteria with genomes that show $\geq 95 \%$ average nucleotide identity (ANI) to the type genome, which has been assigned the MAG ID 913 and which is available via NCBI BioSample SAMN15816858. This is a new name for the alphanumeric GTDB species sp001836165. The GC content of the type genome is $61.16 \%$ and the genome length is $2.1 \mathrm{Mbp}$.

\section{Description of Candidatus Corynebacterium gallistercoris sp. nov.}

Candidatus Corynebacterium gallistercoris (gal.li.ster'co.ris. L. masc. n. gallus chicken; L. neut. n. stercus dung; N.L. gen. n. gallistercoris of chicken faeces)

A bacterial species identified by metagenomic analyses. This species includes all bacteria with genomes that show $\geq 95 \%$ average nucleotide identity (ANI) to the type genome, which has been assigned the MAG ID 4376 and which is available via NCBI BioSample SAMN15816747. The $\mathrm{GC}$ content of the type genome is $62.96 \%$ and the genome length is $2.0 \mathrm{Mbp}$.

\section{Description of Candidatus Corynebacterium intestinavium sp. nov.}

Candidatus Corynebacterium intestinavium (in.tes.tin.a'vi.um. L. neut. n. intestinum gut; L. fem. n. avis bird; N.L. gen. n. intestinavium of the gut of birds)

A bacterial species identified by metagenomic analyses. This species includes all bacteria with genomes that show $\geq 95 \%$ average nucleotide identity (ANI) to the type genome, which has been assigned the MAG ID 5925 and which is available via NCBI BioSample SAMN15816787. The $\mathrm{GC}$ content of the type genome is $65.59 \%$ and the genome length is $1.9 \mathrm{Mbp}$.

\section{Description of Candidatus Cottocaccamicrobium gen. nov.}

Candidatus Cottocaccamicrobium (Cot.to.cac.co.mi.cro'bi.um. Gr. masc. n. kottos chicken; Gr. fem. n. kakke faeces; N.L. neut. n. microbium a microbe; N.L. neut. n. Cottocaccamicrobium a microbe asociated with chicken faeces)

Peer) reviewing PDF | (2020:12:55980:1:1:NEW 13 Jan 2021) 
2883 A bacterial genus identified by metagenomic analyses. The genus includes all bacteria with 2884 genomes that show $\geq 60 \%$ average amino acid identity (AAI) to the type genome from the type 2885 species Cottocaccamicrobium excrementipullorum. This genus was identified but not named by 2886 Glendinning et al. This genus has been assigned by GTDB-Tk v1.3.0 working on GTDB Release 2887 05-RS95 (Chaumeil et al., 2019; Parks et al., 2020) to the order Lachnospirales and to the family 2888 Lachnospiraceae.

2889 2890

\section{Description of Candidatus Cottocaccamicrobium excrementipullorum sp. nov.} Candidatus Cottocaccamicrobium excrementipullorum (ex.cre.men.ti.pul.lo'rum. L. neut. n. excrementum excrement; L. masc. n. pullus a young chicken; N.L. gen. n. excrementipullorum of young chicken excrement)

A bacterial species identified by metagenomic analyses. This species includes all bacteria with genomes that show $\geq 95 \%$ average nucleotide identity (ANI) to the type genome, which has been assigned the MAG ID CHK179-5732 and which is available via NCBI BioSample SAMN15816932. The GC content of the type genome is $47.54 \%$ and the genome length is 3.4 Mbp.

\section{Description of Candidatus Cryptobacteroides gen. nov.}

Candidatus Cryptobacteroides (Cryp.to.bac.te.ro'i.des. Gr. masc. adj. kryptos hidden; N.L. masc. n. Bacteroides a genus name; N.L. masc. n. Cryptobacteroides a genus related to the genus Bacteroides but distinct from it)

A bacterial genus identified by metagenomic analyses. The genus includes all bacteria with genomes that show $\geq 60 \%$ average amino acid identity (AAI) to the type genome from the type species Cryptobacteroides avicola. This is a name for the alphanumeric GTDB genus RC9. This genus has been assigned by GTDB-Tk v1.3.0 working on GTDB Release 05-RS95 (Chaumeil et al., 2019; Parks et al., 2020) to the order Bacteroidales and to the family UBA932.

\section{Description of Candidatus Cryptobacteroides avicola sp. nov.}

Candidatus Cryptobacteroides avicola (a.vi'co.la. L. fem. n. avis bird; L. suff. -cola inhabitant of; N.L. n. avicola inhabitant of birds)

A bacterial species identified by metagenomic analyses. This species includes all bacteria with genomes that show $\geq 95 \%$ average nucleotide identity (ANI) to the type genome, which has been assigned the MAG ID G3-8215 and which is available via NCBI BioSample SAMN15817056. The GC content of the type genome is $49.81 \%$ and the genome length is $2.6 \mathrm{Mbp}$.

\section{Description of Candidatus Cryptobacteroides avistercoris sp. nov.}

Candidatus Cryptobacteroides avistercoris (a.vi.ster'co.ris. L. fem. n. avis bird; L. neut. n. stercus dung; N.L. gen. n. avistercoris of bird faeces) 
2929 A bacterial species identified by metagenomic analyses. This species includes all bacteria with

2930

2931

2932

2933

2934

2935

2936

2937

2938

2939

2940

2941

2942

2943

2944

2945

2946

2947

2948

2949

2950

2951

2952

2953

2954

2955

2956

2957

2958

2959

2960

2961

2962

2963

2964

2965

2966

2967

2968

2969

2970

2971

2972

2973 genomes that show $\geq 95 \%$ average nucleotide identity (ANI) to the type genome, which has been assigned the MAG ID B3-1481 and which is available via NCBI BioSample SAMN15817057. The GC content of the type genome is $59.28 \%$ and the genome length is $1.6 \mathrm{Mbp}$.

\section{Description of Candidatus Cryptobacteroides excrementavium sp. nov.} Candidatus Cryptobacteroides excrementavium (ex.cre.ment.a'vi.um. L. neut. n. excrementum excrement; L. fem. n. avis bird; N.L. gen. n. excrementavium of bird excrement)

A bacterial species identified by metagenomic analyses. This species includes all bacteria with genomes that show $\geq 95 \%$ average nucleotide identity (ANI) to the type genome, which has been assigned the MAG ID B2-16538 and which is available via NCBI BioSample SAMN15817059. The GC content of the type genome is $50.29 \%$ and the genome length is $2.1 \mathrm{Mbp}$.

\section{Description of Candidatus Cryptobacteroides excrementigallinarum sp. nov.}

Candidatus Cryptobacteroides excrementigallinarum (ex.cre.men.ti.gal.li.na'rum. L. neut. n. excrementum excrement; L. fem. n. gallina hen; N.L. gen. n. excrementigallinarum of hen excrement)

A bacterial species identified by metagenomic analyses. This species includes all bacteria with genomes that show $\geq 95 \%$ average nucleotide identity (ANI) to the type genome, which has been assigned the MAG ID ChiHecolR1B25-7735 and which is available via NCBI BioSample SAMN15817167. This is a new name for the alphanumeric GTDB species sp900543205. The $\mathrm{GC}$ content of the type genome is $57.80 \%$ and the genome length is $1.8 \mathrm{Mbp}$.

\section{Description of Candidatus Cryptobacteroides excrementipullorum sp. nov. Candidatus Cryptobacteroides excrementipullorum (ex.cre.men.ti.pul.lo'rum. L. neut. n. excrementum excrement; L. masc. gen.pl. n. pullorum of young chickens; N.L. gen. $\mathrm{n}$. excrementipullorum of young chicken excrement)}

A bacterial species identified by metagenomic analyses. This species includes all bacteria with genomes that show $\geq 95 \%$ average nucleotide identity (ANI) to the type genome, which has been assigned the MAG ID 2478 and which is available via NCBI BioSample SAMN15817061. The GC content of the type genome is $52.35 \%$ and the genome length is $2.5 \mathrm{Mbp}$.

\section{Description of Candidatus Cryptobacteroides faecavium sp. nov.} Candidatus Cryptobacteroides faecavium (faec.a'vi.um. L. fem. n. faex, faecis excrement; L. fem. n. avis bird; N.L. gen. n. faecavium of bird faeces)

A bacterial species identified by metagenomic analyses. This species includes all bacteria with genomes that show $\geq 95 \%$ average nucleotide identity (ANI) to the type genome, which has been 
2974

2975

2976

2977

2978

2979

2980

2981

2982

2983

2984

2985

2986

2987

2988

2989

2990

2991

2992

2993

2994

2995

2996

2997

2998

2999

3000

3001

3002

3003

3004

3005

3006

3007

3008

3009

3010

3011

3012

3013

3014

3015

3016

3017

3018

3019

assigned the MAG ID B2-22910 and which is available via NCBI BioSample SAMN15817063. The GC content of the type genome is $52.41 \%$ and the genome length is $2.4 \mathrm{Mbp}$.

\author{
Description of Candidatus Cryptobacteroides faecigallinarum sp. nov. \\ Candidatus Cryptobacteroides faecigallinarum (fae.ci.gal.li.na'rum. L. fem. n. faex, faecis \\ excrement; L. fem. n. gallina hen; N.L. gen. n. faecigallinarum of hen faeces)
}

A bacterial species identified by metagenomic analyses. This species includes all bacteria with genomes that show $\geq 95 \%$ average nucleotide identity (ANI) to the type genome, which has been assigned the MAG ID B1-13419 and which is available via NCBI BioSample SAMN15817072. The GC content of the type genome is $49.87 \%$ and the genome length is $2.0 \mathrm{Mbp}$.

\title{
Description of Candidatus Cryptobacteroides faecipullorum sp. nov.
}

Candidatus Cryptobacteroides faecipullorum (fae.ci.pul.lo'rum. L. fem. n. faex, faecis

excrement; L. masc. n. pullus a young chicken; N.L. gen. n. faecipullorum of young chicken

faeces)

A bacterial species identified by metagenomic analyses. This species includes all bacteria with genomes that show $\geq 95 \%$ average nucleotide identity (ANI) to the type genome, which has been assigned the MAG ID B1-15692 and which is available via NCBI BioSample SAMN15817080. The GC content of the type genome is $49.55 \%$ and the genome length is $2.3 \mathrm{Mbp}$.

\section{Description of Candidatus Cryptobacteroides gallistercoris sp. nov.}

Candidatus Cryptobacteroides gallistercoris (gal.li.ster'co.ris. L. masc. $\mathrm{n}$ gallus chicken; L. neut. n. stercus dung; N.L. gen. n. gallistercoris of chicken faeces)

A bacterial species identified by metagenomic analyses. This species includes all bacteria with genomes that show $\geq 95 \%$ average nucleotide identity (ANI) to the type genome, which has been assigned the MAG ID F1-3629 and which is available via NCBI BioSample SAMN15817088. The GC content of the type genome is $51.79 \%$ and the genome length is $2.0 \mathrm{Mbp}$.

\section{Description of Candidatus Cryptobacteroides intestinavium sp. nov.}

Candidatus Cryptobacteroides intestinavium (in.tes.tin.a'vi.um. L. neut. n. intestinum gut; L. fem. n. avis bird; N.L. gen. n. intestinavium of the gut of birds)

A bacterial species identified by metagenomic analyses. This species includes all bacteria with genomes that show $\geq 95 \%$ average nucleotide identity (ANI) to the type genome, which has been assigned the MAG ID B1-20833 and which is available via NCBI BioSample SAMN15817087. The GC content of the type genome is $51.51 \%$ and the genome length is $2.4 \mathrm{Mbp}$.

\section{Description of Candidatus Cryptobacteroides intestinigallinarum sp. nov.}


3020 Candidatus Cryptobacteroides intestinigallinarum (in.tes.ti.ni.gal.li.na'rum. L. neut. n. intestinum

3021

3022

3023

3024

3025

3026

3027

3028

3029

3030

3031

3032

3033

3034

3035

3036

3037

3038

3039

3040

3041

3042

3043

3044

3045

3046

3047

3048

3049

3050

3051

3052

3053

3054

3055

3056

3057

3058

3059

3060

3061

3062

3063

3064

3065

gut; L. fem. n. gallina hen; N.L. gen. n. intestinigallinarum of the gut of the hens)

A bacterial species identified by metagenomic analyses. This species includes all bacteria with genomes that show $\geq 95 \%$ average nucleotide identity (ANI) to the type genome, which has been assigned the MAG ID B1-3475 and which is available via NCBI BioSample SAMN15817089. The GC content of the type genome is $49.55 \%$ and the genome length is $2.3 \mathrm{Mbp}$.

\section{Description of Candidatus Cryptobacteroides intestinipullorum sp. nov.}

Candidatus Cryptobacteroides intestinipullorum (in.tes.ti.ni.pul.lo'rum. L. neut. n. intestinum gut; L. masc. n. pullus a young chicken; N.L. gen. n. intestinipullorum of the gut of young chickens)

A bacterial species identified by metagenomic analyses. This species includes all bacteria with genomes that show $\geq 95 \%$ average nucleotide identity (ANI) to the type genome, which has been assigned the MAG ID 33258 and which is available via NCBI BioSample SAMN15817090. The $\mathrm{GC}$ content of the type genome is $50.60 \%$ and the genome length is $2.4 \mathrm{Mbp}$.

\section{Description of Candidatus Cryptobacteroides merdavium sp. nov.}

Candidatus Cryptobacteroides merdavium (merd.a'vi.um. L. fem. n. merda faeces; L. fem. n. avis bird; N.L. gen. n. merdavium of bird faeces)

A bacterial species identified by metagenomic analyses. This species includes all bacteria with genomes that show $\geq 95 \%$ average nucleotide identity (ANI) to the type genome, which has been assigned the MAG ID D5-748 and which is available via NCBI BioSample SAMN15817104. The GC content of the type genome is $50.98 \%$ and the genome length is $2.5 \mathrm{Mbp}$.

\section{Description of Candidatus Cryptobacteroides merdigallinarum sp. nov.}

Candidatus Cryptobacteroides merdigallinarum (mer.di.gal.li.na'rum. L. fem. n. merda faeces; L. fem. n. gallina hen; N.L. gen. n. merdigallinarum of hen faeces)

A bacterial species identified by metagenomic analyses. This species includes all bacteria with genomes that show $\geq 95 \%$ average nucleotide identity (ANI) to the type genome, which has been assigned the MAG ID 20514 and which is available via NCBI BioSample SAMN15817110. The $\mathrm{GC}$ content of the type genome is $54.69 \%$ and the genome length is $2.3 \mathrm{Mbp}$.

\section{Description of Candidatus Cryptobacteroides merdipullorum sp. nov.}

Candidatus Cryptobacteroides merdipullorum (mer.di.pul.lo'rum. L. fem. n. merda faeces; L. masc. n. pullus a young chicken; N.L. gen. n. merdipullorum of the faeces of young chickens)

A bacterial species identified by metagenomic analyses. This species includes all bacteria with genomes that show $\geq 95 \%$ average nucleotide identity (ANI) to the type genome, which has been

Peer] reviewing PDF | (2020:12:55980:1:1:NEW 13 Jan 2021) 
3066 assigned the MAG ID ChiHecec2B26-709 and which is available via NCBI BioSample

3067 SAMN15817116. The GC content of the type genome is $57.16 \%$ and the genome length is 2.0

3068 Mbp.

3069

3070

3071

3072

Description of Candidatus Cryptobacteroides pullicola sp. nov.

3073

Candidatus Cryptobacteroides pullicola (pul.li'co.la. L. masc. n. pullus a young chicken; L. suff.

3074

3075

3076

3077

3078

3079

-cola inhabitant of; N.L. n. pullicola an inhabitant of young chickens)

A bacterial species identified by metagenomic analyses. This species includes all bacteria with genomes that show $\geq 95 \%$ average nucleotide identity (ANI) to the type genome, which has been assigned the MAG ID ChiHecec2B26-3624 and which is available via NCBI BioSample SAMN15817179. This is a new name for the alphanumeric GTDB species sp001915575. The $\mathrm{GC}$ content of the type genome is $58.01 \%$ and the genome length is $1.7 \mathrm{Mbp}$.

3080

3081

3082

Description of Candidatus Desulfovibrio faecigallinarum sp. nov.

3083

3084

3085

3086

3087

3088

3089

3090

3091

Candidatus Desulfovibrio faecigallinarum (fae.ci.gal.li.na'rum. L. fem. n. faex, faecis excrement; L. fem. n. gallina hen; N.L. gen. n. faecigallinarum of hen faeces)

A bacterial species identified by metagenomic analyses. This species includes all bacteria with genomes that show $\geq 95 \%$ average nucleotide identity (ANI) to the type genome, which has been assigned the MAG ID 8923 and which is available via NCBI BioSample SAMN15816873. This is a new name for the alphanumeric GTDB species sp002159665. The GC content of the type genome is $57.29 \%$ and the genome length is $2.0 \mathrm{Mbp}$.

3092

3093

3094

Description of Candidatus Desulfovibrio gallistercoris sp. nov.

Candidatus Desulfovibrio gallistercoris (gal.li.ster'co.ris. L. masc. n. gallus chicken; L. neut. n.

3095

stercus dung; N.L. gen. n. gallistercoris of chicken faeces)

3096

3097

A bacterial species identified by metagenomic analyses. This species includes all bacteria with

3098

3099 genomes that show $\geq 95 \%$ average nucleotide identity (ANI) to the type genome, which has been assigned the MAG ID ChiGjej2B2-32749 and which is available via NCBI BioSample

3100 SAMN15816654. The GC content of the type genome is $64.54 \%$ and the genome length is 2.8

3101 Mbp.

3102

3103

3104

3105

\section{Description of Candidatus Desulfovibrio intestinavium sp. nov.}

Candidatus Desulfovibrio intestinavium (in.tes.tin.a'vi.um. L. neut. n. intestinum gut; L. fem. n.

3106

3107

3108

3109

3110 avis bird; N.L. gen. n. intestinavium of the gut of birds)

A bacterial species identified by metagenomic analyses. This species includes all bacteria with genomes that show $\geq 95 \%$ average nucleotide identity (ANI) to the type genome, which has been assigned the MAG ID 5032 and which is available via NCBI BioSample SAMN15816664. The

3111 GC content of the type genome is $64.60 \%$ and the genome length is $2.5 \mathrm{Mbp}$. 
Description of Candidatus Desulfovibrio intestinigallinarum sp. nov.

3115 Candidatus Desulfovibrio intestinigallinarum (in.tes.ti.ni.gal.li.na'rum. L. neut. n. intestinum gut;

3116 L. fem. n. gallina hen; N.L. gen. n. intestinigallinarum of the gut of the hens)

3117

3118

3119

3120

3121

3122

3123

3124

3125

3126

3127

3128

3129

3130

3131

3132

3133

3134

3135

3136

3137

A bacterial species identified by metagenomic analyses. This species includes all bacteria with genomes that show $\geq 95 \%$ average nucleotide identity (ANI) to the type genome, which has been assigned the MAG ID ChiHecec3B27-2601 and which is available via NCBI BioSample SAMN15816737. The GC content of the type genome is $61.03 \%$ and the genome length is 2.9 Mbp.

\section{Description of Candidatus Desulfovibrio intestinipullorum sp. nov.}

Candidatus Desulfovibrio intestinipullorum (in.tes.ti.ni.pul.lo'rum. L. neut. n. intestinum gut; L. masc. n. pullus a young chicken; N.L. gen. n. intestinipullorum of the gut of young chickens)

A bacterial species identified by metagenomic analyses. This species includes all bacteria with genomes that show $\geq 95 \%$ average nucleotide identity (ANI) to the type genome, which has been assigned the MAG ID ChiHecec2B26-446 and which is available via NCBI BioSample SAMN15816774. The GC content of the type genome is $60.23 \%$ and the genome length is 2.8 Mbp.

\section{Description of Candidatus Dietzia intestinigallinarum sp. nov.}

Candidatus Dietzia intestinigallinarum (in.tes.ti.ni.gal.li.na'rum. L. neut. n. intestinum gut; L.

3138

3139

3140

3141

3142

3143

3144

3145

3146 fem. n. gallina hen; N.L. gen. n. intestinigallinarum of the gut of the hens)

A bacterial species identified by metagenomic analyses. This species includes all bacteria with genomes that show $\geq 95 \%$ average nucleotide identity (ANI) to the type genome, which has been assigned the MAG ID ChiHjej12B11-1528 and which is available via NCBI BioSample SAMN15816635. The GC content of the type genome is $69.86 \%$ and the genome length is 3.9 Mbp.

\section{Description of Candidatus Dietzia intestinipullorum sp. nov.}

Candidatus Dietzia intestinipullorum (in.tes.ti.ni.pul.lo'rum. L. neut. n. intestinum gut; L. masc. n. pullus a young chicken; N.L. gen. n. intestinipullorum of the gut of young chickens)

A bacterial species identified by metagenomic analyses. This species includes all bacteria with genomes that show $\geq 95 \%$ average nucleotide identity (ANI) to the type genome, which has been assigned the MAG ID ChiHjej13B12-8321 and which is available via NCBI BioSample SAMN15816639. The GC content of the type genome is $71.26 \%$ and the genome length is 3.0 Mbp. 
3158 Description of Candidatus Dietzia merdigallinarum sp. nov.

3159 Candidatus Dietzia merdigallinarum (mer.di.gal.li.na'rum. L. fem. n. merda faeces; L. fem. n.

3160 gallina hen; N.L. gen. n. merdigallinarum of hen faeces)

3161

3162

3163

A bacterial species identified by metagenomic analyses. This species includes all bacteria with genomes that show $\geq 95 \%$ average nucleotide identity (ANI) to the type genome, which has been

3164

3165

3166 assigned the MAG ID ChiHjej8B7-16427 and which is available via NCBI BioSample SAMN15816758. The GC content of the type genome is $68.76 \%$ and the genome length is 3.6 Mbp.

Description of Candidatus Dorea faecigallinarum sp. nov.

3170 Candidatus Dorea faecigallinarum (fae.ci.gal.li.na'rum. L. fem. n. faex, faecis excrement; L. fem. n. gallina hen; N.L. gen. n. faecigallinarum of hen faeces)

A bacterial species identified by metagenomic analyses. This species includes all bacteria with genomes that show $\geq 95 \%$ average nucleotide identity (ANI) to the type genome, which has been

3175

3176

3177 assigned the MAG ID ChiHjej12B11-29902 and which is available via NCBI BioSample SAMN15816646. The GC content of the type genome is $50.13 \%$ and the genome length is 2.0 Mbp.

3178

3179

3180

Description of Candidatus Dorea faecipullorum sp. nov.

3181 Candidatus Dorea faecipullorum (fae.ci.pul.lo'rum. L. fem. n. faex, faecis excrement; L. masc. n. pullus a young chicken; N.L. gen. n. faecipullorum of young chicken faeces)

A bacterial species identified by metagenomic analyses. This species includes all bacteria with genomes that show $\geq 95 \%$ average nucleotide identity (ANI) to the type genome, which has been assigned the MAG ID ChiGjej2B2-10896 and which is available via NCBI BioSample SAMN15816847. This is a new name for the alphanumeric GTDB species sp900543315. The $\mathrm{GC}$ content of the type genome is $45.40 \%$ and the genome length is $2.3 \mathrm{Mbp}$.

3189

3190

\section{Description of Candidatus Dorea gallistercoris sp. nov.}

3192 Candidatus Dorea gallistercoris (gal.li.ster'co.ris. L. masc. n. gallus chicken; L. neut. n. stercus dung; N.L. gen. n. gallistercoris of chicken faeces)

A bacterial species identified by metagenomic analyses. This species includes all bacteria with genomes that show $\geq 95 \%$ average nucleotide identity (ANI) to the type genome, which has been assigned the MAG ID ChiSxjej1B13-11762 and which is available via NCBI BioSample SAMN15816753. The GC content of the type genome is $51.71 \%$ and the genome length is 2.2 Mbp.

\section{Description of Candidatus Dorea intestinavium sp. nov.}


3203 Candidatus Dorea intestinavium (in.tes.tin.a'vi.um. L. neut. n. intestinum gut; L. fem. n. avis

3204 bird; N.L. gen. n. intestinavium of the gut of birds)

3205

3206

3207

3208

3209

3210

3211

3212

3213

3214

3215

3216

3217

3218

3219

3220

3221

3222

3223

3224

3225

3226

3227

3228

3229

3230

3231

3232

3233

3234

3235

3236

3237

3238

3239

3240

3241

3242

3243

3244

3245

3246

3247

A bacterial species identified by metagenomic analyses. This species includes all bacteria with genomes that show $\geq 95 \%$ average nucleotide identity (ANI) to the type genome, which has been assigned the MAG ID CHK160-14747 and which is available via NCBI BioSample SAMN15816767. The GC content of the type genome is $35.91 \%$ and the genome length is 1.9 Mbp.

\section{Description of Candidatus Dorea intestinigallinarum sp. nov.}

Candidatus Dorea intestinigallinarum (in.tes.ti.ni.gal.li.na'rum. L. neut. n. intestinum gut; L. fem. n. gallina hen; N.L. gen. n. intestinigallinarum of the gut of the hens)

A bacterial species identified by metagenomic analyses. This species includes all bacteria with genomes that show $\geq 95 \%$ average nucleotide identity (ANI) to the type genome, which has been assigned the MAG ID CHK188-17839 and which is available via NCBI BioSample SAMN15816854. This is a new name for the alphanumeric GTDB species sp000765215. The $\mathrm{GC}$ content of the type genome is $54.58 \%$ and the genome length is $2.5 \mathrm{Mbp}$.

\section{Description of Candidatus Dorea merdavium sp. nov.}

Candidatus Dorea merdavium (merd.a'vi.um. L. fem. n. merda faeces; L. fem. n. avis bird; N.L. gen. n. merdavium of bird faeces)

A bacterial species identified by metagenomic analyses. This species includes all bacteria with genomes that show $\geq 95 \%$ average nucleotide identity (ANI) to the type genome, which has been assigned the MAG ID ChiSxjej1B13-1060 and which is available via NCBI BioSample SAMN15816851. This is a new name for the alphanumeric GTDB species sp900312975. The GC content of the type genome is $53.23 \%$ and the genome length is $2.0 \mathrm{Mbp}$.

\section{Description of Candidatus Dorea stercoravium sp. nov.}

Candidatus Dorea stercoravium (ster.cor.a'vi.um. L. neut. n. stercus dung; L. fem. n. avis bird; N.L. gen. n. stercoravium of bird faeces)

A bacterial species identified by metagenomic analyses. This species includes all bacteria with genomes that show $\geq 95 \%$ average nucleotide identity (ANI) to the type genome, which has been assigned the MAG ID ChiSjej1B19-6982 and which is available via NCBI BioSample

SAMN15816837. This is a new name for the alphanumeric GTDB species sp002160985. The GC content of the type genome is $55.00 \%$ and the genome length is $2.5 \mathrm{Mbp}$.

\section{Description of Candidatus Duodenibacillus intestinavium sp. nov.}

Candidatus Duodenibacillus intestinavium (in.tes.tin.a'vi.um. L. neut. n. intestinum gut; L. fem.

3248

n. avis bird; N.L. gen. n. intestinavium of the gut of birds)

Peer] reviewing PDF | (2020:12:55980:1:1:NEW 13 Jan 2021) 
A bacterial species identified by metagenomic analyses. This species includes all bacteria with genomes that show $\geq 95 \%$ average nucleotide identity (ANI) to the type genome, which has been assigned the MAG ID 2430 and which is available via NCBI BioSample SAMN15816841. This is a new name for the alphanumeric GTDB species sp900538905. The GC content of the type genome is $55.01 \%$ and the genome length is $1.8 \mathrm{Mbp}$.

\section{Description of Candidatus Duodenibacillus intestinigallinarum sp. nov.}

Candidatus Duodenibacillus intestinigallinarum (in.tes.ti.ni.gal.li.na'rum. L. neut. n. intestinum gut; L. fem. n. gallina hen; N.L. gen. n. intestinigallinarum of the gut of the hens)

A bacterial species identified by metagenomic analyses. This species includes all bacteria with genomes that show $\geq 95 \%$ average nucleotide identity (ANI) to the type genome, which has been assigned the MAG ID CHK1-2119 and which is available via NCBI BioSample SAMN15816840. This is a new name for the alphanumeric GTDB species sp003472385. The GC content of the type genome is $56.00 \%$ and the genome length is $2.0 \mathrm{Mbp}$.

\section{Description of Candidatus Egerieenecus gen. nov.}

Candidatus Egerieenecus (E.ger.i.een.e'cus. L. fem. n. egeries dung; Gr. masc. enoikos inhabitant; N.L. masc. n. Egerieenecus a microbe associated with faeces)

A bacterial genus identified by metagenomic analyses. The genus includes all bacteria with genomes that show $\geq 60 \%$ average amino acid identity (AAI) to the type genome from the type species Egerieenecus merdigallinarum. This is a name for the alphanumeric GTDB genus UMGS1600. This genus has been assigned by GTDB-Tk v1.3.0 working on GTDB Release 05RS95 (Chaumeil et al., 2019; Parks et al., 2020) to the order Christensenellales and to the family CAG-74.

\section{Description of Candidatus Egerieenecus merdigallinarum sp. nov.} Candidatus Egerieenecus merdigallinarum (mer.di.gal.li.na'rum. L. fem. n. merda faeces; L. fem. n. gallina hen; N.L. gen. n. merdigallinarum of hen faeces)

A bacterial species identified by metagenomic analyses. This species includes all bacteria with genomes that show $\geq 95 \%$ average nucleotide identity (ANI) to the type genome, which has been assigned the MAG ID ChiSxjej2B14-4419 and which is available via NCBI BioSample SAMN15817218. This is a new name for the alphanumeric GTDB species sp900553295. The $\mathrm{GC}$ content of the type genome is $60.15 \%$ and the genome length is $2.5 \mathrm{Mbp}$.

\section{Description of Candidatus Egerieicola gen. nov.}

Candidatus Egerieicola (E.ger.i.ei.co'la. L. fem. n. egeries dung; L. suff. -cola inhabitant of; N.L. fem. n. Egerieicola a microbe associated with faeces) 
3295 A bacterial genus identified by metagenomic analyses. The genus includes all bacteria with 3296 genomes that show $\geq 60 \%$ average amino acid identity (AAI) to the type genome from the type

3297

3298

3299

3300

3301

3302

3303

3304

3305

3306

3307

3308

3309

3310

3311

3312

3313

3314

3315

3316

3317

3318

3319

3320

3321

3322

3323

3324

3325

3326

3327

3328

3329

3330

3331

3332

3333

3334

3335

3336

3337

3338

3339

3340

species Egerieicola faecale. This is a name for the alphanumeric GTDB genus UBA1375. This genus has been assigned by GTDB-Tk v1.3.0 working on GTDB Release 05-RS95 (Chaumeil et al., 2019; Parks et al., 2020) to the order Oscillospirales and to the family Ruminococcaceae.

\section{Description of Candidatus Egerieicola faecale sp. nov. Candidatus Egerieicola faecale (fae.ca'le. L. neut. adj.faecale of faeces)}

A bacterial species identified by metagenomic analyses. This species includes all bacteria with genomes that show $\geq 95 \%$ average nucleotide identity (ANI) to the type genome, which has been assigned the MAG ID 4509 and which is available via NCBI BioSample SAMN15817200. This is a new name for the alphanumeric GTDB species sp002305795. The GC content of the type genome is $55.66 \%$ and the genome length is $1.8 \mathrm{Mbp}$.

\section{Description of Candidatus Egerieicola pullicola sp. nov.}

Candidatus Egerieicola pullicola (pul.li'co.la. L. masc. n. pullus a young chicken; L. suff. -cola inhabitant of; N.L. n. pullicola an inhabitant of young chickens)

A bacterial species identified by metagenomic analyses. This species includes all bacteria with genomes that show $\geq 95 \%$ average nucleotide identity (ANI) to the type genome, which has been assigned the MAG ID CHK184-25365 and which is available via NCBI BioSample SAMN15817017. The GC content of the type genome is $52.81 \%$ and the genome length is 1.9 Mbp.

\section{Description of Candidatus Egerieimonas gen. nov.}

Candidatus Egerieimonas (E.ger.i.eimo'nas. L. fem. n. egeries dung; L. fem. n. monas a monad; N.L. fem. n. Egerieimonas a microbe associated with faeces)

A bacterial genus identified by metagenomic analyses. The genus includes all bacteria with genomes that show $\geq 60 \%$ average amino acid identity (AAI) to the type genome from the type species Egerieimonas intestinavium. This is a name for the alphanumeric GTDB genus UMGS1472. This genus has been assigned by GTDB-Tk v1.3.0 working on GTDB Release 05RS95 (Chaumeil et al., 2019; Parks et al., 2020) to the order Lachnospirales and to the family Lachnospiraceae.

\section{Description of Candidatus Egerieimonas faecigallinarum sp. nov.}

Candidatus Egerieimonas faecigallinarum (fae.ci.gal.li.na'rum. L. fem. n. faex, faecis excrement; L. fem. n. gallina hen; N.L. gen. n. faecigallinarum of hen faeces)

A bacterial species identified by metagenomic analyses. This species includes all bacteria with genomes that show $\geq 95 \%$ average nucleotide identity (ANI) to the type genome, which has been 
3341 assigned the MAG ID CHK180-10209 and which is available via NCBI BioSample

3342 SAMN15817015. The GC content of the type genome is $51.75 \%$ and the genome length is 2.9

3343 Mbp.

3344

3345

3346

3347

3348

3349

3350

3351

3352

3353

3354

3355

3356

3357

3358

3359

3360

3361

3362

3363

3364

3365

3366

\section{Description of Candidatus Egerieimonas intestinavium sp. nov.}

Candidatus Egerieimonas intestinavium (chicken faceces intestinum gut; L. fem. n. avis bird; N.L. gen. n. intestinavium of the gut of birds)

A bacterial species identified by metagenomic analyses. This species includes all bacteria with genomes that show $\geq 95 \%$ average nucleotide identity (ANI) to the type genome, which has been assigned the MAG ID ChiSxjej1B13-7041 and which is available via NCBI BioSample SAMN15817079. The GC content of the type genome is $55.23 \%$ and the genome length is 2.6 Mbp.

\section{Description of Candidatus Egerieisoma gen. nov.}

Candidatus Egerieisoma (E.ger.i.ei.so'ma. L. fem. n. egeries dung; Gr. neut. n. soma a body; N.L. neut. n. Egerieisoma a microbe associated with faeces)

A bacterial genus identified by metagenomic analyses. The genus includes all bacteria with genomes that show $\geq 60 \%$ average amino acid identity (AAI) to the type genome from the type species Egerieisoma faecipullorum. This is a name for the alphanumeric GTDB genus UMGS1537. This genus has been assigned by GTDB-Tk v1.3.0 working on GTDB Release 05RS95 (Chaumeil et al., 2019; Parks et al., 2020) to the order UBA1212 and to the family UBA1255.

3367

3368

3369

3370

3371

3372

3373

3374

3375

3376

3377

3378

3379

3380

3381

3382

3383

3384

3385

3386

\section{Description of Candidatus Egerieisoma faecipullorum sp. nov.}

Candidatus Egerieisoma faecipullorum (fae.ci.pul.lo'rum. L. fem. n. faex, faecis excrement; L. masc. n. pullus a young chicken; N.L. gen. n. faecipullorum of young chicken faeces)

A bacterial species identified by metagenomic analyses. This species includes all bacteria with genomes that show $\geq 95 \%$ average nucleotide identity (ANI) to the type genome, which has been assigned the MAG ID CHK195-4489 and which is available via NCBI BioSample SAMN15817230. This is a new name for the alphanumeric GTDB species sp900543695. The $\mathrm{GC}$ content of the type genome is $50.69 \%$ and the genome length is $2.0 \mathrm{Mbp}$.

\section{Description of Candidatus Egerieousia gen. nov.}

Candidatus Egerieousia (E.ger.i.e.ou'si.a. L. fem. n. egeries dung; Gr. fem. n. ousia an essence; N.L. fem. n. Egerieousia a microbe associated with faeces)

A bacterial genus identified by metagenomic analyses. The genus includes all bacteria with genomes that show $\geq 60 \%$ average amino acid identity (AAI) to the type genome from the type species Egerieousia excrementavium. This is a name for the alphanumeric GTDB genus 
3387 UBA1232. This genus has been assigned by GTDB-Tk v1.3.0 working on GTDB Release 05-

3388 RS95 (Chaumeil et al., 2019; Parks et al., 2020) to the order Bacteroidales and to the family

3389 UBA932.

3390

3391

3392

3393

3394

3395

3396

3397

3398

3399

3400

3401

3402

3403

3404

3405

3406

3407

3408

3409

3410

3411

3412

3413

3414

3415

3416

3417

3418

3419

3420

3421

3422

3423

3424

3425

3426

3427

3428

3429

3430

3431

3432

\begin{abstract}
Description of Candidatus Egerieousia excrementavium sp. nov.
Candidatus Egerieousia excrementavium (ex.cre.ment.a'vi.um. L. neut. n. excrementum excrement; L. fem. n. avis bird; N.L. gen. n. excrementavium of bird excrement)
\end{abstract}

A bacterial species identified by metagenomic analyses. This species includes all bacteria with genomes that show $\geq 95 \%$ average nucleotide identity (ANI) to the type genome, which has been assigned the MAG ID 15467 and which is available via NCBI BioSample SAMN15817149. The $\mathrm{GC}$ content of the type genome is $46.96 \%$ and the genome length is $1.5 \mathrm{Mbp}$.

\title{
Description of Candidatus Eisenbergiella intestinigallinarum sp. nov.
}

Candidatus Eisenbergiella intestinigallinarum (in.tes.ti.ni.gal.li.na'rum. L. neut. n. intestinum gut; L. fem. n. gallina hen; N.L. gen. n. intestinigallinarum of the gut of the hens)

A bacterial species identified by metagenomic analyses. This species includes all bacteria with genomes that show $\geq 95 \%$ average nucleotide identity (ANI) to the type genome, which has been assigned the MAG ID ChiBcec1-1630 and which is available via NCBI BioSample SAMN15816806. This is a new name for the alphanumeric GTDB species sp900544445. The $\mathrm{GC}$ content of the type genome is $53.15 \%$ and the genome length is $3.4 \mathrm{Mbp}$.

\section{Description of Candidatus Eisenbergiella intestinipullorum sp. nov.}

Candidatus Eisenbergiella intestinipullorum (in.tes.ti.ni.pul.lo'rum. L. neut. n. intestinum gut; L. masc. n. pullus a young chicken; N.L. gen. n. intestinipullorum of the gut of young chickens)

A bacterial species identified by metagenomic analyses. This species includes all bacteria with genomes that show $\geq 95 \%$ average nucleotide identity (ANI) to the type genome, which has been assigned the MAG ID CHK177-9469 and which is available via NCBI BioSample

SAMN15816580. The GC content of the type genome is $54.63 \%$ and the genome length is 3.5 Mbp.

\section{Description of Candidatus Eisenbergiella merdavium sp. nov.}

Candidatus Eisenbergiella merdavium (merd.a'vi.um. L. fem. n. merda faeces; L. fem. n. avis bird; N.L. gen. n. merdavium of bird faeces)

A bacterial species identified by metagenomic analyses. This species includes all bacteria with genomes that show $\geq 95 \%$ average nucleotide identity (ANI) to the type genome, which has been assigned the MAG ID USAMLcec2-132 and which is available via NCBI BioSample SAMN15816641. The GC content of the type genome is $54.07 \%$ and the genome length is 4.2 Mbp. 
3433

3434

3435

3436

3437

3438

3439

3440

3441

3442

3443

3444

3445

3446

3447

3448

3449

3450

3451

3452

3453

3454

3455

3456

3457

3458

3459

3460

3461

3462

3463

3464

3465

3466

3467

3468

3469

3470

3471

3472

3473

3474

3475

3476

3477

3478
Description of Candidatus Eisenbergiella merdigallinarum sp. nov.

Candidatus Eisenbergiella merdigallinarum (mer.di.gal.li.na'rum. L. fem. n. merda faeces; L. fem. n. gallina hen; N.L. gen. n. merdigallinarum of hen faeces)

A bacterial species identified by metagenomic analyses. This species includes all bacteria with genomes that show $\geq 95 \%$ average nucleotide identity (ANI) to the type genome, which has been assigned the MAG ID USAMLcec3-2134 and which is available via NCBI BioSample

SAMN15816643. The GC content of the type genome is $57.03 \%$ and the genome length is 3.2 Mbp.

\section{Description of Candidatus Eisenbergiella merdipullorum sp. nov.}

Candidatus Eisenbergiella merdipullorum (mer.di.pul.lo'rum. L. fem. n. merda faeces; L. masc. n. pullus a young chicken; N.L. gen. n. merdipullorum of the faeces of young chickens)

A bacterial species identified by metagenomic analyses. This species includes all bacteria with genomes that show $\geq 95 \%$ average nucleotide identity (ANI) to the type genome, which has been assigned the MAG ID CHK179-7159 and which is available via NCBI BioSample SAMN15816597. The GC content of the type genome is $51.92 \%$ and the genome length is 3.5 Mbp.

\section{Description of Candidatus Eisenbergiella pullicola sp. nov.}

Candidatus Eisenbergiella pullicola (pul.li'co.la. L. masc. n. pullus a young chicken; L. suff. cola inhabitant of; N.L. n. pullicola an inhabitant of young chickens)

A bacterial species identified by metagenomic analyses. This species includes all bacteria with genomes that show $\geq 95 \%$ average nucleotide identity (ANI) to the type genome, which has been assigned the MAG ID CHK197-24098 and which is available via NCBI BioSample

SAMN15816836. This is a new name for the alphanumeric GTDB species sp003343625. The $\mathrm{GC}$ content of the type genome is $54.54 \%$ and the genome length is $2.6 \mathrm{Mbp}$.

\section{Description of Candidatus Eisenbergiella pullistercoris sp. nov.}

Candidatus Eisenbergiella pullistercoris (pul.li.ster'co.ris. L. masc. n. pullus a young chicken; L. neut. n. stercus dung; N.L. gen. n. pullistercoris of young chicken faeces)

A bacterial species identified by metagenomic analyses. This species includes all bacteria with genomes that show $\geq 95 \%$ average nucleotide identity (ANI) to the type genome, which has been assigned the MAG ID ChiSxjej3B15-24422 and which is available via NCBI BioSample SAMN15816711. The GC content of the type genome is $56.23 \%$ and the genome length is 3.3 Mbp. 
3479 Description of Candidatus Eisenbergiella stercoravium sp. nov.

3480 Candidatus Eisenbergiella stercoravium (ster.cor.a'vi.um. L. neut. n. stercus dung; L. fem. n.

3481

3482

3483

3484

3485

3486

3487

3488

3489

3490

3491

3492

3493

3494

3495

3496

3497

3498

3499

avis bird; N.L. gen. n. stercoravium of bird faeces)

A bacterial species identified by metagenomic analyses. This species includes all bacteria with genomes that show $\geq 95 \%$ average nucleotide identity (ANI) to the type genome, which has been assigned the MAG ID USAMLcec4-2206 and which is available via NCBI BioSample SAMN15816624. The GC content of the type genome is $51.92 \%$ and the genome length is 3.9 Mbp.

\section{Description of Candidatus Eisenbergiella stercorigallinarum sp. nov.}

Candidatus Eisenbergiella stercorigallinarum (ster.co.ri.gal.li.na'rum. L. neut. n. stercus dung; L. fem. n. gallina hen; N.L. gen. n. stercorigallinarum of hen faeces)

A bacterial species identified by metagenomic analyses. This species includes all bacteria with genomes that show $\geq 95 \%$ average nucleotide identity (ANI) to the type genome, which has been assigned the MAG ID ChiHjej8B7-25341 and which is available via NCBI BioSample SAMN15816792. The GC content of the type genome is $55.79 \%$ and the genome length is 2.8 Mbp.

3500

3501

3502

3503

3504

3505

3506

3507

3508

3509

3510

3511

3512

3513

Description of Candidatus Enterenecus gen. nov.

Candidatus Enterenecus (En.ter.en.e'cus. Gr. neut. n. enteron the gut; Gr. masc. enoikos

inhabitant; N.L. masc. n. Enterenecus a microbe associated with the intestines)

A bacterial genus identified by metagenomic analyses. The genus includes all bacteria with genomes that show $\geq 60 \%$ average amino acid identity (AAI) to the type genome from the type species Enterenecus merdae. This is a name for the alphanumeric GTDB genus UBA9475. This genus has been assigned by GTDB-Tk v1.3.0 working on GTDB Release 05-RS95 (Chaumeil et al., 2019; Parks et al., 2020) to the order Oscillospirales and to the family Oscillospiraceae.

\section{Description of Candidatus Enterenecus avicola sp. nov. \\ Candidatus Enterenecus avicola (a.vi'co.la. L. fem. n. avis bird; L. suff. -cola inhabitant of; N.L. n. avicola inhabitant of birds) \\ A bacterial species identified by metagenomic analyses. This species includes all bacteria with genomes that show $\geq 95 \%$ average nucleotide identity (ANI) to the type genome, which has been assigned the MAG ID 153 and which is available via NCBI BioSample SAMN15817108. The $\mathrm{GC}$ content of the type genome is $60.43 \%$ and the genome length is $1.9 \mathrm{Mbp}$.}

3515

3516

3517

3518

3519

3520

3521

3522

3523

\footnotetext{
Description of Candidatus Enterenecus avistercoris sp. nov. Candidatus Enterenecus avistercoris (a.vi.ster'co.ris. L. fem. n. avis bird; L. neut. n. stercus dung; N.L. gen. n. avistercoris of bird faeces)
} 
3525

3526

3527

3528

3529

3530

3531

3532

3533

3534

3535

3536

3537

3538

3539

3540

3541

3542

3543

3544

3545

3546

3547

3548

3549

3550

3551

3552

3553

3554

3555

3556

3557

3558

3559

3560

3561

3562

3563

3564

3565

3566

3567

3568

3569

3570

A bacterial species identified by metagenomic analyses. This species includes all bacteria with genomes that show $\geq 95 \%$ average nucleotide identity (ANI) to the type genome, which has been assigned the MAG ID ChiSxjej3B15-11837 and which is available via NCBI BioSample SAMN15817165. The GC content of the type genome is $64.30 \%$ and the genome length is 1.5 Mbp.

\section{Description of Candidatus Enterenecus faecium sp. nov.}

Candidatus Enterenecus faecium (fae'ci.um. L. fem. n. faex, faecis excrement; L. masc. gen.pl. n. faecium of faeces)

A bacterial species identified by metagenomic analyses. This species includes all bacteria with genomes that show $\geq 95 \%$ average nucleotide identity (ANI) to the type genome, which has been assigned the MAG ID ChiGjej2B2-12916 and which is available via NCBI BioSample SAMN15817211. This is a new name for the alphanumeric GTDB species sp002161675. The $\mathrm{GC}$ content of the type genome is $60.00 \%$ and the genome length is $2.0 \mathrm{Mbp}$.

\section{Description of Candidatus Enterenecus merdae sp. nov. \\ Candidatus Enterenecus merdae (mer'dae. L. gen. n. merdae of faeces)}

A bacterial species identified by metagenomic analyses. This species includes all bacteria with genomes that show $\geq 95 \%$ average nucleotide identity (ANI) to the type genome, which has been assigned the MAG ID ChiHcolR17-2730 and which is available via NCBI BioSample SAMN15817102. The GC content of the type genome is $63.48 \%$ and the genome length is 1.7 Mbp.

\section{Description of Candidatus Enterenecus stercoripullorum sp. nov.}

Candidatus Enterenecus stercoripullorum (ster.co.ri.pul.lo'rum. L. neut. n. stercus dung; L. masc. n. pullus a young chicken; N.L. gen. n. stercoripullorum of the faceces of young chickens)

A bacterial species identified by metagenomic analyses. This species includes all bacteria with genomes that show $\geq 95 \%$ average nucleotide identity (ANI) to the type genome, which has been assigned the MAG ID 3668 and which is available via NCBI BioSample SAMN15817106. The $\mathrm{GC}$ content of the type genome is $60.97 \%$ and the genome length is $1.8 \mathrm{Mbp}$.

\section{Description of Candidatus Enterocloster excrementigallinarum sp. nov.}

Candidatus Enterocloster excrementigallinarum (ex.cre.men.ti.gal.li.na'rum. L. neut. n. excrementum excrement; L. fem. n. gallina hen; N.L. gen. n. excrementigallinarum of hen excrement)

A bacterial species identified by metagenomic analyses. This species includes all bacteria with genomes that show $\geq 95 \%$ average nucleotide identity (ANI) to the type genome, which has been

Peer) reviewing PDF | (2020:12:55980:1:1:NEW 13 Jan 2021) 
3571 assigned the MAG ID CHK198-12963 and which is available via NCBI BioSample

3572 SAMN15816811. This is a new name for the alphanumeric GTDB species sp900547035. The

3573 GC content of the type genome is $51.32 \%$ and the genome length is $3.1 \mathrm{Mbp}$.

3574

3575

3576

3577

3578

3579

3580

3581

3582

3583

3584

3585

3586

3587

3588

3589

3590

3591

3592

3593

3594

3595

3596

3597

3598

3599

3600

3601

3602

3603

3604

3605

3606

3607

\section{Description of Candidatus Enterocloster excrementipullorum sp. nov.}

Candidatus Enterocloster excrementipullorum (ex.cre.men.ti.pul.lo'rum. L. neut. n. excrementum excrement; L. masc. n. pullus a young chicken; N.L. gen. n. excrementipullorum of young chicken excrement)

A bacterial species identified by metagenomic analyses. This species includes all bacteria with genomes that show $\geq 95 \%$ average nucleotide identity (ANI) to the type genome, which has been assigned the MAG ID CHK180-15479 and which is available via NCBI BioSample SAMN15816584. The GC content of the type genome is $53.85 \%$ and the genome length is 2.9 Mbp.

\section{Description of Candidatus Enterocloster faecavium sp. nov.}

Candidatus Enterocloster faecavium (faec.a'vi.um. L. fem. n. faex, faecis excrement; L. fem. n. avis bird; N.L. gen. n. faecavium of bird faeces)

A bacterial species identified by metagenomic analyses. This species includes all bacteria with genomes that show $\geq 95 \%$ average nucleotide identity (ANI) to the type genome, which has been assigned the MAG ID CHK188-4685 and which is available via NCBI BioSample SAMN15816596. The GC content of the type genome is $52.23 \%$ and the genome length is 2.8 Mbp.

\section{Description of Candidatus Enterococcus avicola sp. nov.}

Candidatus Enterococcus avicola (a.vi'co.la. L. fem. n. avis bird; L. suff. -cola inhabitant of; N.L. n. avicola inhabitant of birds)

A bacterial species identified by metagenomic analyses. This species includes all bacteria with genomes that show $\geq 95 \%$ average nucleotide identity (ANI) to the type genome, which has been assigned the MAG ID CHK172-16539 and which is available via NCBI BioSample SAMN15816900. Although GTDB has assigned this species to the genus it calls Enterococcus_I

3608

3609

3610

3611

3612

3613

3614 , this genus designation cannot be incorporated into a well-formed binomial, so in naming this species, we have used the current validly published name for the genus. The GC content of the type genome is $36.87 \%$ and the genome length is $2.2 \mathrm{Mbp}$.

3615

\section{Description of Candidatus Enterococcus stercoravium sp. nov.}

Candidatus Enterococcus stercoravium (ster.cor.a'vi.um. L. neut. n. stercus dung; L. fem. n. avis bird; N.L. gen. n. stercoravium of bird faeces) 
3616 A bacterial species identified by metagenomic analyses. This species includes all bacteria with 3617 genomes that show $\geq 95 \%$ average nucleotide identity (ANI) to the type genome, which has been 3618 assigned the MAG ID CHK172-14336 and which is available via NCBI BioSample

3619 SAMN15816907. Although GTDB has assigned this species to the genus it calls

3620 Enterococcus_C, this genus designation cannot be incorporated into a well-formed binomial, so

3621

3622

3623

3624

3625

3626

3627

3628

3629

3630

3631

3632

3633

3634

3635

3636

3637

3638

3639

3640

3641

3642

3643

3644

3645

3646

3647

3648

3649

3650

3651

3652

3653

3654

3655

3656

3657

3658

3659

3660 in naming this species, we have used the current validly published name for the genus. The GC content of the type genome is $44.16 \%$ and the genome length is $2.3 \mathrm{Mbp}$.

\section{Description of Candidatus Enterococcus stercoripullorum sp. nov.}

Candidatus Enterococcus stercoripullorum (ster.co.ri.pul.lo'rum. L. neut. n. stercus dung; L. masc. n. pullus a young chicken; N.L. gen. n. stercoripullorum of the faceces of young chickens)

A bacterial species identified by metagenomic analyses. This species includes all bacteria with genomes that show $\geq 95 \%$ average nucleotide identity (ANI) to the type genome, which has been assigned the MAG ID ChiHjej12B11-924 and which is available via NCBI BioSample SAMN15816914. Although GTDB has assigned this species to the genus it calls Enterococcus_E , this genus designation cannot be incorporated into a well-formed binomial, so in naming this species, we have used the current validly published name for the genus. The GC content of the type genome is $36.20 \%$ and the genome length is $2.3 \mathrm{Mbp}$.

\section{Description of Candidatus Enterocola gen. nov.}

Candidatus Enterocola (En.ter.o.co'la. Gr. neut. n. enteron the gut; L. suff. -cola inhabitant of; N.L. fem. n. Enterocola a microbe associated with faeces)

A bacterial genus identified by metagenomic analyses. The genus includes all bacteria with genomes that show $\geq 60 \%$ average amino acid identity (AAI) to the type genome from the type species Enterocola intestinipullorum. This is a name for the alphanumeric GTDB genus RUG163. This genus has been assigned by GTDB-Tk v1.3.0 working on GTDB Release 05RS95 (Chaumeil et al., 2019; Parks et al., 2020) to the order Bacteroidales and to the family Paludibacteraceae.

\section{Description of Candidatus Enterocola intestinipullorum sp. nov.} Candidatus Enterocola intestinipullorum (in.tes.ti.ni.pul.lo'rum. L. neut. n. intestinum gut; L. masc. n. pullus a young chicken; N.L. gen. n. intestinipullorum of the gut of young chickens)

A bacterial species identified by metagenomic analyses. This species includes all bacteria with genomes that show $\geq 95 \%$ average nucleotide identity (ANI) to the type genome, which has been assigned the MAG ID D3-1215 and which is available via NCBI BioSample SAMN15817113. The GC content of the type genome is $47.46 \%$ and the genome length is $1.8 \mathrm{Mbp}$.

\section{Description of Candidatus Enteromonas gen. nov.}


3661 Candidatus Enteromonas (En.ter.omo'nas. Gr. neut. n. enteron the gut; L. fem. n. monas a

3662 monad; N.L. fem. n. Enteromonas a microbe associated with the intestines)

3663

3664

3665

3666

3667

3668

3669

3670

3671

3672

3673

3674

3675

3676

3677

3678

3679

A bacterial genus identified by metagenomic analyses. The genus includes all bacteria with genomes that show $\geq 60 \%$ average amino acid identity (AAI) to the type genome from the type species Enteromonas pullistercoris. This is a name for the alphanumeric GTDB genus UBA733. This genus has been assigned by GTDB-Tk v1.3.0 working on GTDB Release 05-RS95 (Chaumeil et al., 2019; Parks et al., 2020) to the order RFN20 and to the family CAG-826.

\section{Description of Candidatus Enteromonas pullicola sp. nov.}

Candidatus Enteromonas pullicola (pul.li'co.la. L. masc. n. pullus a young chicken; L. suff. -cola inhabitant of; N.L. n. pullicola an inhabitant of young chickens)

A bacterial species identified by metagenomic analyses. This species includes all bacteria with genomes that show $\geq 95 \%$ average nucleotide identity (ANI) to the type genome, which has been assigned the MAG ID ChiGjej1B1-22543 and which is available via NCBI BioSample SAMN15817133. The GC content of the type genome is $57.43 \%$ and the genome length is 1.2 Mbp.

3680

3681

3682

Description of Candidatus Enteromonas pullistercoris sp. nov.

3683 Candidatus Enteromonas pullistercoris (pul.li.ster'co.ris. L. masc. n. pullus a young chicken; L.

3684

3685

3686

3687

3688

3689

3690 neut. n. stercus dung; N.L. gen. n. pullistercoris of young chicken faeces)

A bacterial species identified by metagenomic analyses. This species includes all bacteria with genomes that show $\geq 95 \%$ average nucleotide identity (ANI) to the type genome, which has been assigned the MAG ID 17113 and which is available via NCBI BioSample SAMN15817142. The $\mathrm{GC}$ content of the type genome is $53.99 \%$ and the genome length is $1.4 \mathrm{Mbp}$.

3691

3692

Description of Candidatus Enterosoma gen. nov.

3693

Candidatus Enterosoma (En.ter.o.so'ma. Gr. neut. n. enteron the gut; Gr. neut. n. soma a body;

3694 N.L. neut. n. Enterosoma a microbe associated with the intestines)

A bacterial genus identified by metagenomic analyses. The genus includes all bacteria with genomes that show $\geq 60 \%$ average amino acid identity (AAI) to the type genome from the type species Enterosoma merdigallinarum. This is a name for the alphanumeric GTDB genus UBA7642. This genus has been assigned by GTDB-Tk v1.3.0 working on GTDB Release 05RS95 (Chaumeil et al., 2019; Parks et al., 2020) to the order RFN20 and to the family CAG-288.

3701

3702

3703

Description of Candidatus Enterosoma merdigallinarum sp. nov.

3704

Candidatus Enterosoma merdigallinarum (mer.di.gal.li.na'rum. L. fem. n. merda faeces; L. fem.

3705

3706 
3707 A bacterial species identified by metagenomic analyses. This species includes all bacteria with 3708 genomes that show $\geq 95 \%$ average nucleotide identity (ANI) to the type genome, which has been 3709 assigned the MAG ID 33044 and which is available via NCBI BioSample SAMN15817141. The

3710

3711

3712

3713

3714

3715

3716

3717

3718

3719

3720

3721

3722

3723

3724

3725

3726

3727

3728

3729

3730

3731

3732

3733

3734

3735

3736

3737

3738

3739

3740

3741

3742

3743

3744

3745

3746

3747

3748

3749

3750

3751

3752

\section{Description of Candidatus Enterousia gen. nov.}

Candidatus Enterousia (En.ter.ou'si.a. Gr. neut. n. enteron the gut; Gr. fem. n. ousia an essence; N.L. fem. n. Enterousia a microbe associated with the intestines)

A bacterial genus identified by metagenomic analyses. The genus includes all bacteria with genomes that show $\geq 60 \%$ average amino acid identity (AAI) to the type genome from the type species Enterousia excrementavium. This is a name for the alphanumeric GTDB genus Rs-D84. This genus has been assigned by GTDB-Tk v1.3.0 working on GTDB Release 05-RS95 (Chaumeil et al., 2019; Parks et al., 2020) to the order Rs-D84 and to the family Rs-D84.

\section{Description of Candidatus Enterousia avicola sp. nov.}

Candidatus Enterousia avicola (a.vi'co.la. L. fem. n. avis bird; L. suff. -cola inhabitant of; N.L. n. avicola inhabitant of birds)

A bacterial species identified by metagenomic analyses. This species includes all bacteria with genomes that show $\geq 95 \%$ average nucleotide identity (ANI) to the type genome, which has been assigned the MAG ID CHK136-897 and which is available via NCBI BioSample

SAMN15817144. The GC content of the type genome is $39.01 \%$ and the genome length is 0.9 Mbp.

\section{Description of Candidatus Enterousia avistercoris sp. nov.}

Candidatus Enterousia avistercoris (a.vi.ster'co.ris. L. fem. n. avis bird; L. neut. n. stercus dung; N.L. gen. n. avistercoris of bird faeces)

A bacterial species identified by metagenomic analyses. This species includes all bacteria with genomes that show $\geq 95 \%$ average nucleotide identity (ANI) to the type genome, which has been assigned the MAG ID 8207 and which is available via NCBI BioSample SAMN15817150. The $\mathrm{GC}$ content of the type genome is $43.79 \%$ and the genome length is $0.8 \mathrm{Mbp}$.

\section{Description of Candidatus Enterousia excrementavium sp. nov.}

Candidatus Enterousia excrementavium (ex.cre.ment.a'vi.um. L. neut. n. excrementum excrement; L. fem. n. avis bird; N.L. gen. n. excrementavium of bird excrement)

A bacterial species identified by metagenomic analyses. This species includes all bacteria with genomes that show $\geq 95 \%$ average nucleotide identity (ANI) to the type genome, which has been assigned the MAG ID B1-16210 and which is available via NCBI BioSample SAMN15817158. The GC content of the type genome is $44.26 \%$ and the genome length is $0.9 \mathrm{Mbp}$. 
3753

3754

3755

3756

3757

3758

3759

3760

3761

3762

3763

3764

3765

3766

3767

3768

3769

3770

3771

3772

3773

3774

3775

3776

3777

3778

3779

3780

3781

3782

3783

3784

3785

3786

3787

3788

3789

3790

3791

3792

3793

3794

3795

3796

3797

3798

Description of Candidatus Enterousia intestinigallinarum sp. nov.

Candidatus Enterousia intestinigallinarum (in.tes.ti.ni.gal.li.na'rum. L. neut. n. intestinum gut; L. fem. n. gallina hen; N.L. gen. n. intestinigallinarum of the gut of the hens)

A bacterial species identified by metagenomic analyses. This species includes all bacteria with genomes that show $\geq 95 \%$ average nucleotide identity (ANI) to the type genome, which has been assigned the MAG ID ChiGjej3B3-5194 and which is available via NCBI BioSample

SAMN15817183. This is a new name for the alphanumeric GTDB species sp900546185. The $\mathrm{GC}$ content of the type genome is $45.89 \%$ and the genome length is $0.9 \mathrm{Mbp}$.

\section{Description of Candidatus Erysipelatoclostridium merdavium sp. nov.}

Candidatus Erysipelatoclostridium merdavium (merd.a'vi.um. L. fem. n. merda faeces; L. fem. n. avis bird; N.L. gen. n. merdavium of bird faeces)

A bacterial species identified by metagenomic analyses. This species includes all bacteria with genomes that show $\geq 95 \%$ average nucleotide identity (ANI) to the type genome, which has been assigned the MAG ID ChiGjej1B1-14440 and which is available via NCBI BioSample SAMN15816860. This is a new name for the alphanumeric GTDB species sp002160495. The $\mathrm{GC}$ content of the type genome is $29.32 \%$ and the genome length is $2.6 \mathrm{Mbp}$.

\section{Description of Candidatus Eubacterium avistercoris sp. nov.}

Candidatus Eubacterium avistercoris (a.vi.ster'co.ris. L. fem. n. avis bird; L. neut. n. stercus dung; N.L. gen. n. avistercoris of bird faeces)

A bacterial species identified by metagenomic analyses. This species includes all bacteria with genomes that show $\geq 95 \%$ average nucleotide identity (ANI) to the type genome, which has been assigned the MAG ID CHK192-9172 and which is available via NCBI BioSample SAMN15816888. Although GTDB has assigned this species to the genus it calls Eubacterium_I, this genus designation cannot be incorporated into a well-formed binomial, so in naming this species, we have used the current validly published name for the genus. The GC content of the type genome is $45.90 \%$ and the genome length is $2.6 \mathrm{Mbp}$.

\section{Description of Candidatus Eubacterium faecale sp. nov. \\ Candidatus Eubacterium faecale (fae.ca'le. L. neut. adj. faecale of faeces)}

A bacterial species identified by metagenomic analyses. This species includes all bacteria with genomes that show $\geq 95 \%$ average nucleotide identity (ANI) to the type genome, which has been assigned the MAG ID CHK188-16595 and which is available via NCBI BioSample SAMN15816917. This is a new name for the alphanumeric GTDB species sp000431535. Although GTDB has assigned this species to the genus it calls Eubacterium_R, this genus designation cannot be incorporated into a well-formed binomial, so in naming this species, we

PeerJ reviewing PDF | (2020:12:55980:1:1:NEW 13 Jan 2021) 
3799 have used the current validly published name for the genus. The GC content of the type genome

3800 is $46.56 \%$ and the genome length is $1.8 \mathrm{Mbp}$.

3801

3802

3803

Description of Candidatus Eubacterium faecavium sp. nov.

3804

Candidatus Eubacterium faecavium (faec.a'vi.um. L. fem. n. faex, faecis excrement; L. fem. n.

3805

avis bird; N.L. gen. n. faecavium of bird faeces)

3806

3807

A bacterial species identified by metagenomic analyses. This species includes all bacteria with

3808

3809 genomes that show $\geq 95 \%$ average nucleotide identity (ANI) to the type genome, which has been

3810 assigned the MAG ID ChiHecec3B27-3607 and which is available via NCBI BioSample

3811 SAMN15816921. This is a new name for the alphanumeric GTDB species sp900539845.

3812 Although GTDB has assigned this species to the genus it calls Eubacterium_R, this genus

3813 designation cannot be incorporated into a well-formed binomial, so in naming this species, we have used the current validly published name for the genus. The GC content of the type genome

3814 is $45.56 \%$ and the genome length is $1.9 \mathrm{Mbp}$.

3815

3816

3817

Description of Candidatus Eubacterium faecigallinarum sp. nov.

3818 Candidatus Eubacterium faecigallinarum (fae.ci.gal.li.na'rum. L. fem. n. faex, faecis excrement;

3819

3820

3821

L. fem. n. gallina hen; N.L. gen. n. faecigallinarum of hen faeces)

3822

3823

A bacterial species identified by metagenomic analyses. This species includes all bacteria with genomes that show $\geq 95 \%$ average nucleotide identity (ANI) to the type genome, which has been assigned the MAG ID 8396 and which is available via NCBI BioSample SAMN15816904.

3824

3825

3826

3827 Although GTDB has assigned this species to the genus it calls Eubacterium_R, this genus designation cannot be incorporated into a well-formed binomial, so in naming this species, we have used the current validly published name for the genus. The GC content of the type genome is $43.37 \%$ and the genome length is $1.6 \mathrm{Mbp}$.

3828

3829

3830

3831

Description of Candidatus Eubacterium faecipullorum sp. nov.

Candidatus Eubacterium faecipullorum (fae.ci.pul.lo'rum. L. fem. n. faex, faecis excrement; L.

3832 masc. n. pullus a young chicken; N.L. gen. n. faecipullorum of young chicken faeces)

3833

3834

3835

3836

3837

A bacterial species identified by metagenomic analyses. This species includes all bacteria with genomes that show $\geq 95 \%$ average nucleotide identity (ANI) to the type genome, which has been assigned the MAG ID 421 and which is available via NCBI BioSample SAMN15816928. This is a new name for the alphanumeric GTDB species sp900546785. Although GTDB has assigned

3838

3839 this species to the genus it calls Eubacterium_R, this genus designation cannot be incorporated into a well-formed binomial, so in naming this species, we have used the current validly

3840

3841 published name for the genus. The GC content of the type genome is $47.26 \%$ and the genome length is $1.9 \mathrm{Mbp}$.

3842

3843

3844

\section{Description of Candidatus Eubacterium pullicola sp. nov.}


3845 Candidatus Eubacterium pullicola (pul.li'co.la. L. masc. n. pullus a young chicken; L. suff. -cola 3846 inhabitant of; N.L. n. pullicola inhabitant of young chicken)

3847

3848

3849

3850

3851

3852

3853

3854

3855

3856

3857

3858

3859

3860

3861

3862

3863

3864

3865

3866

3867

3868

3869

3870

3871

3872

3873

3874

3875

3876

3877

3878

3879

3880

3881

3882

3883

3884

3885

3886

3887

3888

3889

3890

A bacterial species identified by metagenomic analyses. This species includes all bacteria with genomes that show $\geq 95 \%$ average nucleotide identity (ANI) to the type genome, which has been assigned the MAG ID ChiHjej12B11-11929 and which is available via NCBI BioSample SAMN15816916. This is a new name for the alphanumeric GTDB species sp900540015. Although GTDB has assigned this species to the genus it calls Eubacterium_M, this genus designation cannot be incorporated into a well-formed binomial, so in naming this species, we have used the current validly published name for the genus. The GC content of the type genome is $41.55 \%$ and the genome length is $1.2 \mathrm{Mbp}$.

\section{Description of Candidatus Evtepia excrementipullorum sp. nov.}

Candidatus Evtepia excrementipullorum (ex.cre.men.ti.pul.lo'rum. L. neut. n. excrementum excrement; L. masc. n. pullus a young chicken; N.L. gen. n. excrementipullorum of young chicken excrement)

A bacterial species identified by metagenomic analyses. This species includes all bacteria with genomes that show $\geq 95 \%$ average nucleotide identity (ANI) to the type genome, which has been assigned the MAG ID ChiSjej3B21-3892 and which is available via NCBI BioSample SAMN15816827. This is a new name for the alphanumeric GTDB species sp900546255. The $\mathrm{GC}$ content of the type genome is $63.06 \%$ and the genome length is $2.0 \mathrm{Mbp}$.

\section{Description of Candidatus Evtepia faecavium sp. nov.}

Candidatus Evtepia faecavium (faec.a'vi.um. L. fem. n. faex, faecis excrement; L. fem. n. avis bird; N.L. gen. n. faecavium of bird faeces)

A bacterial species identified by metagenomic analyses. This species includes all bacteria with genomes that show $\geq 95 \%$ average nucleotide identity (ANI) to the type genome, which has been assigned the MAG ID ChiHecec3B27-8621 and which is available via NCBI BioSample SAMN15816713. The GC content of the type genome is $65.40 \%$ and the genome length is 2.0 Mbp.

\section{Description of Candidatus Evtepia faecigallinarum sp. nov.} Candidatus Evtepia faecigallinarum (fae.ci.gal.li.na'rum. L. fem. n. faex, faecis excrement; L. fem. n. gallina hen; N.L. gen. n. faecigallinarum of hen faeces)

A bacterial species identified by metagenomic analyses. This species includes all bacteria with genomes that show $\geq 95 \%$ average nucleotide identity (ANI) to the type genome, which has been assigned the MAG ID ChiHcec3-601 and which is available via NCBI BioSample SAMN15816724. The GC content of the type genome is $63.50 \%$ and the genome length is 2.4 Mbp. 
Description of Candidatus Excrementavichristensenella gen. nov.

3894 Candidatus Excrementavichristensenella (Ex.cre.ment.a.vi.chris.ten.sen.el'la. L. neut. n. excrementum excrement; L. fem. n. avis bird; N.L. fem. n. Christensenella a genus name; N.L. fem n. Excrementavichristensenella a genus related to the genus Christensenella but distinct from it and found in poultry faeces)

3897

A bacterial genus identified by metagenomic analyses. The genus includes all bacteria with genomes that show $\geq 60 \%$ average amino acid identity (AAI) to the type genome from the type species Excrementavichristensenella intestinipullorum. This genus has been assigned by GTDBTk v1.3.0 working on GTDB Release 05-RS95 (Chaumeil et al., 2019; Parks et al., 2020) to the 3903 order Christensenellales and to the family $C A G-74$.

Description of Candidatus Excrementavichristensenella intestinipullorum sp. nov. Candidatus Excrementavichristensenella intestinipullorum (in.tes.ti.ni.pul.lo'rum. L. neut. n. intestinum gut; L. masc. n. pullus a young chicken; N.L. gen. n. intestinipullorum of the gut of young chickens)

A bacterial species identified by metagenomic analyses. This species includes all bacteria with genomes that show $\geq 95 \%$ average nucleotide identity (ANI) to the type genome, which has been assigned the MAG ID ChiGjej2B2-1688 and which is available via NCBI BioSample SAMN15816955. The GC content of the type genome is $62.99 \%$ and the genome length is 2.8 Mbp.

\section{Description of Candidatus Faecalibacterium avium sp. nov.}

3918 Candidatus Faecalibacterium avium (a'vi.um. L. fem. pl. n. avium of birds)

A bacterial species identified by metagenomic analyses. This species includes all bacteria with genomes that show $\geq 95 \%$ average nucleotide identity (ANI) to the type genome, which has been assigned the MAG ID CHK182-10647 and which is available via NCBI BioSample SAMN15816876. This is a new name for the alphanumeric GTDB species sp002160915. The $\mathrm{GC}$ content of the type genome is $62.96 \%$ and the genome length is $2.2 \mathrm{Mbp}$.

3926

3927

3928

3929

Description of Candidatus Faecalibacterium faecigallinarum sp. nov.

Candidatus Faecalibacterium faecigallinarum (fae.ci.gal.li.na'rum. L. fem. n. faex, faecis excrement; L. fem. n. gallina hen; N.L. gen. n. faecigallinarum of hen faeces)

A bacterial species identified by metagenomic analyses. This species includes all bacteria with genomes that show $\geq 95 \%$ average nucleotide identity (ANI) to the type genome, which has been assigned the MAG ID ChiSjej5B23-2810 and which is available via NCBI BioSample 
Description of Candidatus Faecalibacterium faecipullorum sp. nov.

3939

Candidatus Faecalibacterium faecipullorum (fae.ci.pul.lo'rum. L. fem. n. faex, faecis excrement;

3940

3941

3942

L. masc. n. pullus a young chicken; N.L. gen. n. faecipullorum of young chicken faeces)

3943

3944

A bacterial species identified by metagenomic analyses. This species includes all bacteria with genomes that show $\geq 95 \%$ average nucleotide identity (ANI) to the type genome, which has been assigned the MAG ID ChiHjej9B8-13557 and which is available via NCBI BioSample

3945 SAMN15816651. The GC content of the type genome is $65.75 \%$ and the genome length is 2.1

3946 Mbp.

Description of Candidatus Faecalibacterium gallistercoris sp. nov.

Candidatus Faecalibacterium gallistercoris (gal.li.ster'co.ris. L. masc. n. gallus chicken; L. neut. n. stercus dung; N.L. gen. n. gallistercoris of chicken faeces)

3952

3953

3954

3955

A bacterial species identified by metagenomic analyses. This species includes all bacteria with genomes that show $\geq 95 \%$ average nucleotide identity (ANI) to the type genome, which has been assigned the MAG ID ChiBcec16-3735 and which is available via NCBI BioSample SAMN15816605. The GC content of the type genome is $64.68 \%$ and the genome length is 2.1 Mbp.

3958

3959

3960

Description of Candidatus Faecalibacterium intestinavium sp. nov.

3961

Candidatus Faecalibacterium intestinavium (in.tes.tin.a'vi.um. L. neut. n. intestinum gut; L. fem.

3962

n. avis bird; N.L. gen. n. intestinavium of the gut of birds)

3963

3964

3965

A bacterial species identified by metagenomic analyses. This species includes all bacteria with genomes that show $\geq 95 \%$ average nucleotide identity (ANI) to the type genome, which has been assigned the MAG ID 742 and which is available via NCBI BioSample SAMN15816744. The $\mathrm{GC}$ content of the type genome is $61.60 \%$ and the genome length is $1.8 \mathrm{Mbp}$.

3968

3969

3970

\section{Description of Candidatus Faecalibacterium intestinigallinarum sp. nov.}

3971

Candidatus Faecalibacterium intestinigallinarum (in.tes.ti.ni.gal.li.na'rum. L. neut. n. intestinum

3972

3973

3974 gut; L.fem. n. gallina hen; N.L. gen. n. intestinigallinarum of the gut of the hens)

3975

3976

A bacterial species identified by metagenomic analyses. This species includes all bacteria with genomes that show $\geq 95 \%$ average nucleotide identity (ANI) to the type genome, which has been assigned the MAG ID ChiHcolR34-3080 and which is available via NCBI BioSample

3977

3978 SAMN15816770. The GC content of the type genome is $64.34 \%$ and the genome length is 2.1

3979 Mbp.

\section{Description of Candidatus Faecalibacterium intestinipullorum sp. nov.}


3982 Candidatus Faecalibacterium intestinipullorum (in.tes.ti.ni.pul.lo'rum. L. neut. n. intestinum gut;

3983 L. masc. n. pullus a young chicken; N.L. gen. n. intestinipullorum of the gut of young chickens)

3984

3985

3986

3987

3988

3989

3990

3991

3992

3993

3994

3995

3996

3997

3998

3999

4000

4001

4002

4003

4004

4005

4006

4007

4008

4009

4010

4011

A bacterial species identified by metagenomic analyses. This species includes all bacteria with genomes that show $\geq 95 \%$ average nucleotide identity (ANI) to the type genome, which has been assigned the MAG ID ChiHcolR21-11242 and which is available via NCBI BioSample SAMN15816785. The GC content of the type genome is $61.39 \%$ and the genome length is 2.1 Mbp.

\section{Description of Candidatus Faecalicoccus intestinipullorum sp. nov.}

Candidatus Faecalicoccus intestinipullorum (in.tes.ti.ni.pul.lo'rum. L. neut. n. intestinum gut; L. masc. n. pullus a young chicken; N.L. gen. n. intestinipullorum of the gut of young chickens)

A bacterial species identified by metagenomic analyses. This species includes all bacteria with genomes that show $\geq 95 \%$ average nucleotide identity (ANI) to the type genome, which has been assigned the MAG ID ChiHjej8B7-5959 and which is available via NCBI BioSample SAMN15816766. The GC content of the type genome is $40.89 \%$ and the genome length is 1.4 Mbp.

\section{Description of Candidatus Faecaligallichristensenella gen. nov.}

Candidatus Faecaligallichristensenella (Fae.ca.li.gal.li.chris.ten.sen.el'la. N.L. masc. adj. faecalis pertaining to faeces; L. masc. n. gallus chicken; N.L. fem. n. Christensenella a genus name; N.L. fem. n. Faecaligallichristensenella a genus related to the genus Christensenella but distinct from it and found in poultry faeces)

A bacterial genus identified by metagenomic analyses. The genus includes all bacteria with genomes that show $\geq 60 \%$ average amino acid identity (AAI) to the type genome from the type species Faecaligallichristensenella faecipullorum. This genus was identified but not named by

4012 Glendinning et al. This genus has been assigned by GTDB-Tk v1.3.0 working on GTDB Release 4013 05-RS95 (Chaumeil et al., 2019; Parks et al., 2020) to the order Christensenellales and to the 4014 family $C A G-74$.

4015

4016

Description of Candidatus Faecaligallichristensenella faecipullorum sp. nov.

4018 Candidatus Faecaligallichristensenella faecipullorum (fae.ci.pul.lo'rum. L. fem. n. faex, faecis 4019 excrement; L. masc. n. pullus a young chicken; N.L. gen. n. faecipullorum of young chicken 4020 4021 faeces)

4023

4024

4025

4026

A bacterial species identified by metagenomic analyses. This species includes all bacteria with genomes that show $\geq 95 \%$ average nucleotide identity (ANI) to the type genome, which has been assigned the MAG ID ChiSjej6B24-5839 and which is available via NCBI BioSample SAMN15816940. The GC content of the type genome is $58.49 \%$ and the genome length is 2.6 Mbp. 
4028

4029

4030

4031

4032

4033

4034

4035

4036

4037

4038

4039

4040

4041

4042

4043

4044

4045

4046

4047

4048

4049

4050

4051

4052

4053

4054

4055

4056

4057

4058

4059

4060

4061

4062

4063

4064

4065

4066

4067

4068

4069

4070

4071

4072

\section{Description of Candidatus Faecenecus gen. nov.}

Candidatus Faecenecus (Faecen.e'cus. L. fem. n. faex dregs; Gr. masc. enoikos inhabitant; N.L. masc. n. Faecenecus a microbe associated with faeces)

A bacterial genus identified by metagenomic analyses. The genus includes all bacteria with genomes that show $\geq 60 \%$ average amino acid identity (AAI) to the type genome from the type species Faecenecus gallistercoris. This is a name for the alphanumeric GTDB genus CAG-988. This genus has been assigned by GTDB-Tk v1.3.0 working on GTDB Release 05-RS95 (Chaumeil et al., 2019; Parks et al., 2020) to the order RF39 and to the family CAG-611.

\section{Description of Candidatus Faecenecus gallistercoris sp. nov.}

Candidatus Faecenecus gallistercoris (gal.li.ster'co.ris. L. masc. $\mathrm{n}$ gallus chicken; L. neut. $\mathrm{n}$. stercus dung; N.L. gen. n. gallistercoris of chicken faeces)

A bacterial species identified by metagenomic analyses. This species includes all bacteria with genomes that show $\geq 95 \%$ average nucleotide identity (ANI) to the type genome, which has been assigned the MAG ID CHK165-10780 and which is available via NCBI BioSample

SAMN15817166. This is a new name for the alphanumeric GTDB species sp003149915. The $\mathrm{GC}$ content of the type genome is $34.49 \%$ and the genome length is $1.2 \mathrm{Mbp}$.

\section{Description of Candidatus Faecicola gen. nov.}

Candidatus Faecicola (Faec.i.co'la. L. fem. n. faex dregs; L. suff. -cola inhabitant of; N.L. fem. n. Faecicola a microbe associated with faeces)

A bacterial genus identified by metagenomic analyses. The genus includes all bacteria with genomes that show $\geq 60 \%$ average amino acid identity (AAI) to the type genome from the type species Faecicola pullistercoris. This is a name for the alphanumeric GTDB genus CAG-1138. This genus has been assigned by GTDB-Tk v1.3.0 working on GTDB Release 05-RS95 (Chaumeil et al., 2019; Parks et al., 2020) to the order 4C28d-15 and to the family CAG-917.

\section{Description of Candidatus Faecicola pullistercoris sp. nov.}

Candidatus Faecicola pullistercoris (pul.li.ster'co.ris. L. masc. n. pullus a young chicken; L. fem. n. avis bird; N.L. gen. n. pullistercoris of young chicken faeces)

A bacterial species identified by metagenomic analyses. This species includes all bacteria with genomes that show $\geq 95 \%$ average nucleotide identity (ANI) to the type genome, which has been assigned the MAG ID 5944 and which is available via NCBI BioSample SAMN15817151. The GC content of the type genome is $48.54 \%$ and the genome length is $1.6 \mathrm{Mbp}$.

\section{Description of Candidatus Faecimonas gen. nov.}


4073 Candidatus Faecimonas (Faec.i.mo'nas. L. fem. n. faex dregs; L. fem. n. monas a monad; N.L. 4074 fem. n. Faecimonas a microbe associated with faeces)

4075

4076

4077

4078

4079

4080

4081

4082

4083

4084

4085

4086

4087

4088

4089

4090

4091

4092

4093

4094

4095

4096

4097

4098

4099

4100

4101

4102

4103

4104

4105

4106

4107

4108

4109

4110

4111

4112

4113

4114

4115

4116

4117

A bacterial genus identified by metagenomic analyses. The genus includes all bacteria with genomes that show $\geq 60 \%$ average amino acid identity (AAI) to the type genome from the type species Faecimonas intestinavium. This is a name for the alphanumeric GTDB genus CAG-877. This genus has been assigned by GTDB-Tk v1.3.0 working on GTDB Release 05-RS95 (Chaumeil et al., 2019; Parks et al., 2020) to the order RF39 and to the family CAG-611.

\section{Description of Candidatus Faecimonas gallistercoris sp. nov.}

Candidatus Faecimonas gallistercoris (gal.li.ster'co.ris. L. masc. $\mathrm{n}$ gallus chicken; L. neut. $\mathrm{n}$. stercus dung; N.L. gen. n. gallistercoris of chicken faeces)

A bacterial species identified by metagenomic analyses. This species includes all bacteria with genomes that show $\geq 95 \%$ average nucleotide identity (ANI) to the type genome, which has been assigned the MAG ID CHK189-3136 and which is available via NCBI BioSample SAMN15817016. The GC content of the type genome is $28.18 \%$ and the genome length is 1.4 Mbp.

\section{Description of Candidatus Faecimonas intestinavium sp. nov.}

Candidatus Faecimonas intestinavium (in.tes.tin.a'vi.um. L. neut. n. intestinum gut; L. fem. n. avis bird; N.L. gen. $\mathrm{n}$. intestinavium of the gut of birds)

A bacterial species identified by metagenomic analyses. This species includes all bacteria with genomes that show $\geq 95 \%$ average nucleotide identity (ANI) to the type genome, which has been assigned the MAG ID USAMLcec2-12447 and which is available via NCBI BioSample SAMN15817225. This is a new name for the alphanumeric GTDB species sp900554305. The GC content of the type genome is $29.22 \%$ and the genome length is $1.8 \mathrm{Mbp}$.

\section{Description of Candidatus Faecimorpha gen. nov.}

Candidatus Faecimorpha (Faec.i.mor'pha. L. fem. n. faex dregs; Gr. fem. n. morphe a form, shape; N.L. fem. n. Faecimorpha a microbe associated with faeces)

A bacterial genus identified by metagenomic analyses. The genus includes all bacteria with genomes that show $\geq 60 \%$ average amino acid identity (AAI) to the type genome from the type species Faecimorpha stercoravium. This is a name for the alphanumeric GTDB genus UBA1390. This genus has been assigned by GTDB-Tk v1.3.0 working on GTDB Release 05RS95 (Chaumeil et al., 2019; Parks et al., 2020) to the order Lachnospirales and to the family UBA1390.

\section{Description of Candidatus Faecimorpha stercoravium sp. nov.}


4118 Candidatus Faecimorpha stercoravium (ster.cor.a'vi.um. L. neut. n. stercus dung; L. fem. n. avis 4119 bird; N.L. gen. n. stercoravium of bird faeces)

4120

4121

4122

4123

4124

4125

4126

4127

4128

4129

4130

4131

4132

4133

4134

4135

4136

4137

4138

4139

4140

4141

4142

4143

4144

4145

4146

4147

4148

4149

4150

4151

4152

4153

4154

4155

4156

4157

4158

4159

4160

4161

4162

4163

A bacterial species identified by metagenomic analyses. This species includes all bacteria with genomes that show $\geq 95 \%$ average nucleotide identity (ANI) to the type genome, which has been assigned the MAG ID CHK195-9767 and which is available via NCBI BioSample

SAMN15817172. This is a new name for the alphanumeric GTDB species sp002305315. The $\mathrm{GC}$ content of the type genome is $49.81 \%$ and the genome length is $2.4 \mathrm{Mbp}$.

\section{Description of Candidatus Faeciplasma gen. nov.}

Candidatus Faeciplasma (Faec.i.plas'ma. L. fem. n. faex dregs; Gr. neut. n. plasma a form; N.L. neut. n. Faeciplasma a microbe associated with faeces)

A bacterial genus identified by metagenomic analyses. The genus includes all bacteria with genomes that show $\geq 60 \%$ average amino acid identity (AAI) to the type genome from the type species Faeciplasma avium. This is a name for the alphanumeric GTDB genus UBA1409. This genus has been assigned by GTDB-Tk v1.3.0 working on GTDB Release 05-RS95 (Chaumeil et al., 2019; Parks et al., 2020) to the order Oscillospirales and to the family Ruminococcaceae.

\section{Description of Candidatus Faeciplasma avium sp. nov. \\ Candidatus Faeciplasma avium (a'vi.um. L. fem. pl. n. avium of birds)}

A bacterial species identified by metagenomic analyses. This species includes all bacteria with genomes that show $\geq 95 \%$ average nucleotide identity (ANI) to the type genome, which has been assigned the MAG ID 1370 and which is available via NCBI BioSample SAMN15817208. This is a new name for the alphanumeric GTDB species sp002305045. The GC content of the type genome is $51.56 \%$ and the genome length is $1.6 \mathrm{Mbp}$.

\section{Description of Candidatus Faeciplasma gallinarum sp. nov.}

Candidatus Faeciplasma gallinarum (gal.li.na'rum. L. fem. n. gallina a hen; L. gen.fem. pl. n. gallinarum of hens)

A bacterial species identified by metagenomic analyses. This species includes all bacteria with genomes that show $\geq 95 \%$ average nucleotide identity (ANI) to the type genome, which has been assigned the MAG ID CHK157-1446 and which is available via NCBI BioSample

SAMN15817182. This is a new name for the alphanumeric GTDB species sp002338885. The $\mathrm{GC}$ content of the type genome is $49.59 \%$ and the genome length is $1.6 \mathrm{Mbp}$.

\section{Description of Candidatus Faeciplasma pullistercoris sp. nov.}

Candidatus Faeciplasma pullistercoris (pul.li.ster'co.ris. L. masc. n. pullus a young chicken; L. neut. n. stercus dung; N.L. gen. n. pullistercoris of young chicken faeces)

Peer) reviewing PDF | (2020:12:55980:1:1:NEW 13 Jan 2021) 
4164 A bacterial species identified by metagenomic analyses. This species includes all bacteria with 4165 genomes that show $\geq 95 \%$ average nucleotide identity (ANI) to the type genome, which has been 4166 assigned the MAG ID CHK33-4379 and which is available via NCBI BioSample

4167 SAMN15817120. The GC content of the type genome is $49.45 \%$ and the genome length is 1.5 4168 Mbp.

4169

4170

4171

4172

4173

4174

4175

4176

4177

4178

4179

4180

4181

4182

4183

4184

4185

4186

4187

4188

4189

4190

4191

4192

4193

4194

4195

4196

4197

4198

4199

Description of Candidatus Faecisoma gen. nov.

Candidatus Faecisoma (Faec.i.so'ma. L. fem. n. faex dregs; Gr. neut. n. soma a body; N.L. neut. n. Faecisoma a microbe associated with faeces)

A bacterial genus identified by metagenomic analyses. The genus includes all bacteria with genomes that show $\geq 60 \%$ average amino acid identity (AAI) to the type genome from the type species Faecisoma merdavium. This is a name for the alphanumeric GTDB genus CAG- 878 . This genus has been assigned by GTDB-Tk v1.3.0 working on GTDB Release 05-RS95 (Chaumeil et al., 2019; Parks et al., 2020) to the order RF39 and to the family CAG-822.

\section{Description of Candidatus Faecisoma merdavium sp. nov.}

Candidatus Faecisoma merdavium (merd.a'vi.um. L. fem. n. merda faeces; L. fem. n. avis bird; N.L. gen. n. merdavium of bird faeces)

A bacterial species identified by metagenomic analyses. This species includes all bacteria with genomes that show $\geq 95 \%$ average nucleotide identity (ANI) to the type genome, which has been assigned the MAG ID 6595 and which is available via NCBI BioSample SAMN15817101. The $\mathrm{GC}$ content of the type genome is $24.63 \%$ and the genome length is $1.3 \mathrm{Mbp}$.

\section{Description of Candidatus Faecivicinus gen. nov.}

Candidatus Faecivicinus (Faec.i.vic.in'us. L. fem. n. faex dregs; L. masc. n. vicinus a neighbour; N.L. masc. n. Faecivicinus a microbe associated with faeces)

A bacterial genus identified by metagenomic analyses. The genus includes all bacteria with genomes that show $\geq 60 \%$ average amino acid identity (AAI) to the type genome from the type species Faecivicinus avistercoris. This is a name for the alphanumeric GTDB genus

4200

4201

4202

4203

4204

4205

4206

4207

4208 UMGS1603. This genus has been assigned by GTDB-Tk v1.3.0 working on GTDB Release 05RS95 (Chaumeil et al., 2019; Parks et al., 2020) to the order Christensenellales and to the family CAG-74.

\section{Description of Candidatus Faecivicinus avistercoris sp. nov.}

Candidatus Faecivicinus avistercoris (a.vi.ster'co.ris. L. fem. n. avis bird; L. neut. n. stercus dung; N.L. gen. n. avistercoris of bird faeces)

4209

A bacterial species identified by metagenomic analyses. This species includes all bacteria with genomes that show $\geq 95 \%$ average nucleotide identity (ANI) to the type genome, which has been 
4210 assigned the MAG ID 905 and which is available via NCBI BioSample SAMN15817031. The

4211

4212

4213

4214

4215

4216

4217

4218

4219

4220

4221

4222

4223

4224

4225

4226

4227

4228

4229

4230

4231

4232

4233

4234

4235

4236

4237

4238

4239

4240

4241

4242

4243

4244

4245

4246

4247

4248

4249

4250

4251

4252

4253

4254

4255

$\mathrm{GC}$ content of the type genome is $63.46 \%$ and the genome length is $2.7 \mathrm{Mbp}$.

\section{Description of Candidatus Faecivivens gen. nov.}

Candidatus Faecivivens (Faec.i.viv'ens. L. fem. n. faex dregs; N.L. pres. part. vivens living; N.L. fem. n. Faecivivens a microbe associated with faeces)

A bacterial genus identified by metagenomic analyses. The genus includes all bacteria with genomes that show $\geq 60 \%$ average amino acid identity (AAI) to the type genome from the type species Faecivivens stercorigallinarum. This is a name for the alphanumeric GTDB genus UBA1448. This genus has been assigned by GTDB-Tk v1.3.0 working on GTDB Release 05RS95 (Chaumeil et al., 2019; Parks et al., 2020) to the order Oscillospirales and to the family Ruminococcaceae.

\section{Description of Candidatus Faecivivens stercoravium sp. nov.}

Candidatus Faecivivens stercoravium (ster.cor.a'vi.um. L. neut. n. stercus dung; L. fem. n. avis bird; N.L. gen. n. stercoravium of bird faeces)

A bacterial species identified by metagenomic analyses. This species includes all bacteria with genomes that show $\geq 95 \%$ average nucleotide identity (ANI) to the type genome, which has been assigned the MAG ID CHK189-12415 and which is available via NCBI BioSample SAMN15817018. The GC content of the type genome is $59.75 \%$ and the genome length is 2.3 Mbp.

\section{Description of Candidatus Faecivivens stercorigallinarum sp. nov.}

Candidatus Faecivivens stercorigallinarum (ster.co.ri.gal.li.na'rum. L. neut. n. stercus dung; L. fem. n. gallina hen; N.L. gen. n. stercorigallinarum of hen faeces)

A bacterial species identified by metagenomic analyses. This species includes all bacteria with genomes that show $\geq 95 \%$ average nucleotide identity (ANI) to the type genome, which has been assigned the MAG ID 4960 and which is available via NCBI BioSample SAMN15817121. The $\mathrm{GC}$ content of the type genome is $52.99 \%$ and the genome length is $2.2 \mathrm{Mbp}$.

\section{Description of Candidatus Faecivivens stercoripullorum sp. nov.}

Candidatus Faecivivens stercoripullorum (ster.co.ri.pul.lo'rum. L. neut. n. stercus dung; L. masc. n. pullus a young chicken; N.L. gen. n. stercoripullorum of the faceces of young chickens)

A bacterial species identified by metagenomic analyses. This species includes all bacteria with genomes that show $\geq 95 \%$ average nucleotide identity (ANI) to the type genome, which has been assigned the MAG ID ChiBcec7-5410 and which is available via NCBI BioSample SAMN15817124. The GC content of the type genome is $50.38 \%$ and the genome length is 2.0 Mbp. 
4256

4257

4258

4259

4260

4261

4262

4263

4264

4265

4266

4267

4268

4269

4270

4271

4272

4273

4274

4275

4276

4277

4278

4279

4280

4281

4282

4283

4284

4285

4286

4287

4288

4289

4290

4291

4292

4293

4294

4295

4296

4297

4298

4299

4300

4301

Description of Candidatus Faecousia gen. nov.

Candidatus Faecousia (Faec.ou'si.a. L. fem. n. faex dregs; Gr. fem. n. ousia an essence; N.L. fem. n. Faecousia a microbe associated with faeces)

A bacterial genus identified by metagenomic analyses. The genus includes all bacteria with genomes that show $\geq 60 \%$ average amino acid identity (AAI) to the type genome from the type species Faecousia intestinigallinarum. This is a name for the alphanumeric GTDB genus CAG110. This genus has been assigned by GTDB-Tk v1.3.0 working on GTDB Release 05-RS95 (Chaumeil et al., 2019; Parks et al., 2020) to the order Oscillospirales and to the family Oscillospiraceae.

\section{Description of Candidatus Faecousia excrementigallinarum sp. nov.}

Candidatus Faecousia excrementigallinarum (ex.cre.men.ti.gal.li.na'rum. L. neut. n.

excrementum excrement; L. fem. n. gallina hen; N.L. gen. n. excrementigallinarum of hen excrement)

A bacterial species identified by metagenomic analyses. This species includes all bacteria with genomes that show $\geq 95 \%$ average nucleotide identity (ANI) to the type genome, which has been assigned the MAG ID 13361 and which is available via NCBI BioSample SAMN15817055. The GC content of the type genome is $56.40 \%$ and the genome length is $1.9 \mathrm{Mbp}$.

\section{Description of Candidatus Faecousia excrementipullorum sp. nov.}

Candidatus Faecousia excrementipullorum (ex.cre.men.ti.pul.lo'rum. L. neut. n. excrementum excrement; L. masc. n. pullus a young chicken; N.L. gen. n. excrementipullorum of young chicken excrement)

A bacterial species identified by metagenomic analyses. This species includes all bacteria with genomes that show $\geq 95 \%$ average nucleotide identity (ANI) to the type genome, which has been assigned the MAG ID ChiSxjej6B18-3616 and which is available via NCBI BioSample

SAMN15817060. The GC content of the type genome is $56.19 \%$ and the genome length is 1.7 Mbp.

\section{Description of Candidatus Faecousia faecavium sp. nov.}

Candidatus Faecousia faecavium (faec.a'vi.um. L. fem. n. faex, faecis excrement; L. fem. n. avis bird; N.L. gen. n. faecavium of bird faeces)

A bacterial species identified by metagenomic analyses. This species includes all bacteria with genomes that show $\geq 95 \%$ average nucleotide identity (ANI) to the type genome, which has been assigned the MAG ID ChiBcec21-2751 and which is available via NCBI BioSample SAMN15817064. The GC content of the type genome is $53.76 \%$ and the genome length is 2.4 Mbp. 
4302

4303

4304

4305

4306

4307

4308

4309

4310

4311

4312

4313

4314

4315

4316

4317

4318

4319

4320

4321

4322

4323

4324

4325

4326

4327

4328

4329

4330

4331

4332

4333

4334

4335

4336

4337

4338

4339

4340

4341

4342

4343

4344

4345

4346

4347
Description of Candidatus Faecousia faecigallinarum sp. nov.

Candidatus Faecousia faecigallinarum (fae.ci.gal.li.na'rum. L. fem. n. faex, faecis excrement; L. fem. n. gallina hen; N.L. gen. n. faecigallinarum of hen faeces)

A bacterial species identified by metagenomic analyses. This species includes all bacteria with genomes that show $\geq 95 \%$ average nucleotide identity (ANI) to the type genome, which has been assigned the MAG ID ChiHcolR29-948 and which is available via NCBI BioSample SAMN15817073. The GC content of the type genome is $58.86 \%$ and the genome length is 1.9 Mbp.

\section{Description of Candidatus Faecousia faecipullorum sp. nov.}

Candidatus Faecousia faecipullorum (fae.ci.pul.lo'rum. L. fem. n. faex, faecis excrement; L. masc. n. pullus a young chicken; N.L. gen. n. faecipullorum of young chicken faeces)

A bacterial species identified by metagenomic analyses. This species includes all bacteria with genomes that show $\geq 95 \%$ average nucleotide identity (ANI) to the type genome, which has been assigned the MAG ID ChiHecec2B26-1122 and which is available via NCBI BioSample SAMN15817098. The GC content of the type genome is $55.50 \%$ and the genome length is 1.9 Mbp.

\section{Description of Candidatus Faecousia gallistercoris sp. nov.}

Candidatus Faecousia gallistercoris (gal.li.ster'co.ris. L. masc. n gallus chicken; L. neut. n. stercus dung; N.L. gen. n. gallistercoris of chicken faeces)

A bacterial species identified by metagenomic analyses. This species includes all bacteria with genomes that show $\geq 95 \%$ average nucleotide identity (ANI) to the type genome, which has been assigned the MAG ID 7739 and which is available via NCBI BioSample SAMN15817186. This is a new name for the alphanumeric GTDB species sp900546915. The GC content of the type genome is $58.30 \%$ and the genome length is $1.8 \mathrm{Mbp}$.

\section{Description of Candidatus Faecousia intestinavium sp. nov.}

Candidatus Faecousia intestinavium (in.tes.tin.a'vi.um. L. neut. n. intestinum gut; L. fem. n. avis bird; N.L. gen. n. intestinavium of the gut of birds)

A bacterial species identified by metagenomic analyses. This species includes all bacteria with genomes that show $\geq 95 \%$ average nucleotide identity (ANI) to the type genome, which has been assigned the MAG ID ChiHcec3-9842 and which is available via NCBI BioSample

SAMN15817111. The GC content of the type genome is $57.16 \%$ and the genome length is 2.1 Mbp. 
4348 Description of Candidatus Faecousia intestinigallinarum sp. nov.

4349 Candidatus Faecousia intestinigallinarum (in.tes.ti.ni.gal.li.na'rum. L. neut. n. intestinum gut; L.

4350

4351

4352

4353

4354

4355

4356

4357

4358

4359

Description of Candidatus Fimadaptatus gen. nov.

4360

4361

4362

4363

fem. n. gallina hen; N.L. gen. n. intestinigallinarum of the gut of the hens)

A bacterial species identified by metagenomic analyses. This species includes all bacteria with genomes that show $\geq 95 \%$ average nucleotide identity (ANI) to the type genome, which has been assigned the MAG ID ChiSxjej3B15-29383 and which is available via NCBI BioSample SAMN15817112. The GC content of the type genome is $55.74 \%$ and the genome length is 2.1 Mbp.

4364

4365

4366

4367

4368

Candidatus Fimadaptatus (Fim.adaptatus. L. neut. n. fimum dung; L. past part. masc. adaptatus adapted to; N.L. masc. n. Fimadaptatus a microbe associated with faeces)

A bacterial genus identified by metagenomic analyses. The genus includes all bacteria with genomes that show $\geq 60 \%$ average amino acid identity (AAI) to the type genome from the type species Fimadaptatus faecigallinarum. This is a name for the alphanumeric GTDB genus UMGS1633. This genus has been assigned by GTDB-Tk v1.3.0 working on GTDB Release 05RS95 (Chaumeil et al., 2019; Parks et al., 2020) to the order Christensenellales and to the family $C A G-74$.

4369

4370

4371

Description of Candidatus Fimadaptatus faecigallinarum sp. nov.

4372

Candidatus Fimadaptatus faecigallinarum (fae.ci.gal.li.na'rum. L. fem. n. faex, faecis excrement;

4373

4374

4375

L. fem. n. gallina hen; N.L. gen. n. faecigallinarum of hen faeces)

4376

4377

A bacterial species identified by metagenomic analyses. This species includes all bacteria with genomes that show $\geq 95 \%$ average nucleotide identity (ANI) to the type genome, which has been assigned the MAG ID ChiSxjej2B14-8506 and which is available via NCBI BioSample

4378 SAMN15817140. The GC content of the type genome is $60.44 \%$ and the genome length is 2.8

4379

4380

4381

4382 Mbp.

4383

4384

4385

4386

4387

4388

4389

4390

4391

\section{Description of Candidatus Fimenecus gen. nov.}

Candidatus Fimenecus (Fim.en.e'cus. L. neut. n. fimum dung; Gr. masc. enoikos inhabitant; N.L. masc. n. Fimenecus a microbe associated with faeces)

A bacterial genus identified by metagenomic analyses. The genus includes all bacteria with genomes that show $\geq 60 \%$ average amino acid identity (AAI) to the type genome from the type species Fimenecus excrementigallinarum. This is a name for the alphanumeric GTDB genus CAG-180. This genus has been assigned by GTDB-Tk v1.3.0 working on GTDB Release 05RS95 (Chaumeil et al., 2019; Parks et al., 2020) to the order Oscillospirales and to the family Acutalibacteraceae.

4392

4393

Peer) reviewing PDF | (2020:12:55980:1:1:NEW 13 Jan 2021) 
4394 Description of Candidatus Fimenecus excrementavium sp. nov.

4395 Candidatus Fimenecus excrementavium (ex.cre.ment.a'vi.um. L. neut. n. excrementum

4396 excrement; L. fem. n. avis bird; N.L. gen. n. excrementavium of bird excrement)

4397

4398

4399

A bacterial species identified by metagenomic analyses. This species includes all bacteria with genomes that show $\geq 95 \%$ average nucleotide identity (ANI) to the type genome, which has been

4400 assigned the MAG ID ChiSjej1B19-6168 and which is available via NCBI BioSample

4401

4402 SAMN15817011. The GC content of the type genome is $50.61 \%$ and the genome length is 1.8 Mbp.

4403

4404

4405

4406

4407

4408

4409

4410

4411

4412

4413

4414

4415

4416

4417

4418

4419

4420

4421

4422

4423

4424

4425

4426

4427

4428

4429

Description of Candidatus Fimenecus excrementigallinarum sp. nov.

Candidatus Fimenecus excrementigallinarum (ex.cre.men.ti.gal.li.na'rum. L. neut. n. excrementum excrement; L. fem. n. gallina hen; N.L. gen. n. excrementigallinarum of hen excrement)

A bacterial species identified by metagenomic analyses. This species includes all bacteria with genomes that show $\geq 95 \%$ average nucleotide identity (ANI) to the type genome, which has been assigned the MAG ID ChiGjej1B1-19959 and which is available via NCBI BioSample SAMN15817134. The GC content of the type genome is $60.29 \%$ and the genome length is 1.8 Mbp.

\section{Description of Candidatus Fimenecus stercoravium sp. nov.}

Candidatus Fimenecus stercoravium (ster.cor.a'vi.um. L. neut. n. stercus dung; L. fem. n. avis bird; N.L. gen. n. stercoravium of bird faeces)

A bacterial species identified by metagenomic analyses. This species includes all bacteria with genomes that show $\geq 95 \%$ average nucleotide identity (ANI) to the type genome, which has been assigned the MAG ID ChiHcolR13-3023 and which is available via NCBI BioSample

SAMN15817181. This is a new name for the alphanumeric GTDB species sp002314305. The $\mathrm{GC}$ content of the type genome is $55.43 \%$ and the genome length is $1.9 \mathrm{Mbp}$.

\section{Description of Candidatus Fimicola gen. nov.}

Candidatus Fimicola (Fim.i.co'la. L. neut. n. fimum dung; L. suff. -cola inhabitant of; N.L. fem.

4430

4431

4432

4433

n. Fimicola a microbe associated with faeces)

A bacterial genus identified by metagenomic analyses. The genus includes all bacteria with genomes that show $\geq 60 \%$ average amino acid identity (AAI) to the type genome from the type 4434 4435 4436 4437

4438 species Fimicola merdigallinarum. This is a name for the alphanumeric GTDB genus An114. This genus has been assigned by GTDB-Tk v1.3.0 working on GTDB Release 05-RS95 (Chaumeil et al., 2019; Parks et al., 2020) to the order Lachnospirales and to the family Anaerotignaceae. 
4440 Description of Candidatus Fimicola cottocaccae sp. nov.

4441 Candidatus Fimicola cottocaccae (cot.to.cac'cae. Gr. masc. n. kottos chicken Gr. fem. n. kakke

4442 faeces; N.L. gen. n. cottocaccae of chicken faeces)

4443

4444

4445

4446

4447

4448

4449

4450

4451

4452

4453

4454

4455

4456

4457

4458

4459

A bacterial species identified by metagenomic analyses. This species includes all bacteria with genomes that show $\geq 95 \%$ average nucleotide identity (ANI) to the type genome, which has been assigned the MAG ID ChiW9-1577 and which is available via NCBI BioSample SAMN15817191. This is a new name for the alphanumeric GTDB species sp002161055. The GC content of the type genome is $31.91 \%$ and the genome length is $1.8 \mathrm{Mbp}$.

\section{Description of Candidatus Fimicola merdigallinarum sp. nov.}

Candidatus Fimicola merdigallinarum (mer.di.gal.li.na'rum. L. fem. n. merda faeces; L. fem. n. gallina hen; N.L. gen. n. merdigallinarum of hen faeces)

A bacterial species identified by metagenomic analyses. This species includes all bacteria with genomes that show $\geq 95 \%$ average nucleotide identity (ANI) to the type genome, which has been assigned the MAG ID F6-4510 and which is available via NCBI BioSample SAMN15817136. The GC content of the type genome is $32.46 \%$ and the genome length is $1.8 \mathrm{Mbp}$.

4460

4461

Description of Candidatus Fimihabitans gen. nov.

4462

4463

4464

4465

4466

4467

4468

4469

4470

4471

4472

4473

4474

4475

4476

4477

4478

4479

4480

4481

4482

4483

4484

Candidatus Fimihabitans (Fim.i.hab.it'ans. L. neut. n. fimum dung; L. pres. part. habitans an inhabitant; N.L. fem. n. Fimihabitans a microbe associated with faeces)

A bacterial genus identified by metagenomic analyses. The genus includes all bacteria with genomes that show $\geq 60 \%$ average amino acid identity (AAI) to the type genome from the type species Fimihabitans intestinipullorum. This is a name for the alphanumeric GTDB genus UMGS1648. This genus has been assigned by GTDB-Tk v1.3.0 working on GTDB Release 05RS95 (Chaumeil et al., 2019; Parks et al., 2020) to the order RF39 and to the family CAG-822.

\section{Description of Candidatus Fimihabitans intestinipullorum sp. nov.}

Candidatus Fimihabitans intestinipullorum (in.tes.ti.ni.pul.lo'rum. L. neut. n. intestinum gut; L. masc. n. pullus a young chicken; N.L. gen. n. intestinipullorum of the gut of young chickens)

A bacterial species identified by metagenomic analyses. This species includes all bacteria with genomes that show $\geq 95 \%$ average nucleotide identity (ANI) to the type genome, which has been assigned the MAG ID CHK197-8231 and which is available via NCBI BioSample

SAMN15817229. This is a new name for the alphanumeric GTDB species sp900553765. The GC content of the type genome is $33.44 \%$ and the genome length is $1.3 \mathrm{Mbp}$.

\section{Description of Candidatus Fimimonas gen. nov.}

Candidatus Fimimonas (Fim.i.mo'nas. L. neut. n. fimum dung; L. fem. n. monas a monad; N.L. 4485

fem. n. Fimimonas a microbe associated with faeces) 
4486

4487

4488

4489

4490

4491

4492

4493

4494

4495

4496

4497

4498

4499

4500

4501

4502

4503

4504

4505

4506

4507

4508

4509

4510

4511

4512

4513

4514

4515

4516

4517

4518

4519

4520

4521

4522

4523

4524

4525

4526

4527

4528

4529

4530

4531

A bacterial genus identified by metagenomic analyses. The genus includes all bacteria with genomes that show $\geq 60 \%$ average amino acid identity (AAI) to the type genome from the type species Fimimonas gallinarum. This is a name for the alphanumeric GTDB genus CAG-1435. This genus has been assigned by GTDB-Tk v1.3.0 working on GTDB Release 05-RS95 (Chaumeil et al., 2019; Parks et al., 2020) to the order Christensenellales and to the family $C A G$ 314.

Description of Candidatus Fimimonas gallinarum sp. nov.

Candidatus Fimimonas gallinarum (gal.li.na'rum. L. fem. n. gallina a hen; L. gen.fem. pl. n. gallinarum of hens)

A bacterial species identified by metagenomic analyses. This species includes all bacteria with genomes that show $\geq 95 \%$ average nucleotide identity (ANI) to the type genome, which has been assigned the MAG ID CHK121-14286 and which is available via NCBI BioSample

SAMN15817176. This is a new name for the alphanumeric GTDB species sp000433775. The $\mathrm{GC}$ content of the type genome is $45.96 \%$ and the genome length is $1.4 \mathrm{Mbp}$.

\section{Description of Candidatus Fimimonas merdipullorum sp. nov.}

Candidatus Fimimonas merdipullorum (mer.di.pul.lo'rum. L. fem. n. merda faeces; L. masc. n. pullus a young chicken; N.L. gen. n. merdipullorum of the faeces of young chickens)

A bacterial species identified by metagenomic analyses. This species includes all bacteria with genomes that show $\geq 95 \%$ average nucleotide identity (ANI) to the type genome, which has been assigned the MAG ID ChiHjej12B11-7776 and which is available via NCBI BioSample SAMN15817153. The GC content of the type genome is $53.17 \%$ and the genome length is 1.3 Mbp.

\section{Description of Candidatus Fimimorpha gen. nov.}

Candidatus Fimimorpha (Fim.i.mor'pha. L. neut. n. fimum dung; Gr. fem. n. morphe a form, shape; N.L. fem. n. Fimimorpha a microbe associated with faeces)

A bacterial genus identified by metagenomic analyses. The genus includes all bacteria with genomes that show $\geq 60 \%$ average amino acid identity (AAI) to the type genome from the type species Fimimorpha faecalis. This is a name for the alphanumeric GTDB genus CHKCI001. This genus has been assigned by GTDB-Tk v1.3.0 working on GTDB Release 05-RS95 (Chaumeil et al., 2019; Parks et al., 2020) to the order Lachnospirales and to the family Lachnospiraceae.

\section{Description of Candidatus Fimimorpha excrementavium sp. nov.}

Candidatus Fimimorpha excrementavium (ex.cre.ment.a'vi.um. L. neut. n. excrementum excrement; L. fem. n. avis bird; N.L. gen. n. excrementavium of bird excrement)

Peer] reviewing PDF | (2020:12:55980:1:1:NEW 13 Jan 2021) 
4532

4533

4534

4535

4536

4537

4538

4539

4540

4541

4542

4543

4544

4545

4546

4547

4548

4549

4550

4551

4552

4553

4554

4555

4556

4557

4558

4559

4560

4561

4562

4563

4564

4565

4566

4567

4568

4569

4570

4571

4572

4573

4574

4575

4576
A bacterial species identified by metagenomic analyses. This species includes all bacteria with genomes that show $\geq 95 \%$ average nucleotide identity (ANI) to the type genome, which has been assigned the MAG ID CHK193-21555 and which is available via NCBI BioSample

SAMN15817029. The GC content of the type genome is $48.70 \%$ and the genome length is 3.1 Mbp.

\section{Description of Candidatus Fimimorpha faecalis sp. nov. \\ Candidatus Fimimorpha faecalis (fae.ca'lis. L. fem. adj. faecalis of faeces)}

A bacterial species identified by metagenomic analyses. This species includes all bacteria with genomes that show $\geq 95 \%$ average nucleotide identity (ANI) to the type genome, which has been assigned the MAG ID ChiW13-3771 and which is available via NCBI BioSample

SAMN15817177. This is a new name for the alphanumeric GTDB species sp900045905. The $\mathrm{GC}$ content of the type genome is $36.24 \%$ and the genome length is $2.9 \mathrm{Mbp}$.

\section{Description of Candidatus Fimiplasma gen. nov.}

Candidatus Fimiplasma (Fim.i.plas'ma. L. neut. n. fimum dung; Gr. neut. n. plasma a form; N.L. neut. n. Fimiplasma a microbe associated with faeces)

A bacterial genus identified by metagenomic analyses. The genus includes all bacteria with genomes that show $\geq 60 \%$ average amino acid identity (AAI) to the type genome from the type species Fimiplasma intestinipullorum. This is a name for the alphanumeric GTDB genus CHKCI006. This genus has been assigned by GTDB-Tk v1.3.0 working on GTDB Release 05RS95 (Chaumeil et al., 2019; Parks et al., 2020) to the order Erysipelotrichales and to the family Erysipelatoclostridiaceae.

\section{Description of Candidatus Fimiplasma intestinipullorum sp. nov.} Candidatus Fimiplasma intestinipullorum (in.tes.ti.ni.pul.lo'rum. L. neut. n. intestinum gut; L. masc. n. pullus a young chicken; N.L. gen. n. intestinipullorum of the gut of young chickens)

A bacterial species identified by metagenomic analyses. This species includes all bacteria with genomes that show $\geq 95 \%$ average nucleotide identity (ANI) to the type genome, which has been assigned the MAG ID CHK195-11698 and which is available via NCBI BioSample SAMN15817196. This is a new name for the alphanumeric GTDB species sp900018345. The $\mathrm{GC}$ content of the type genome is $43.31 \%$ and the genome length is $2.5 \mathrm{Mbp}$.

\section{Description of Candidatus Fimisoma gen. nov.}

Candidatus Fimisoma (Fim.i.so'ma. L. neut. n. fimum dung; Gr. neut. n. soma a body; N.L. neut. n. Fimisoma a microbe associated with faeces) 
4577 A bacterial genus identified by metagenomic analyses. The genus includes all bacteria with 4578 genomes that show $\geq 60 \%$ average amino acid identity (AAI) to the type genome from the type

4579

4580

4581

4582 species Fimisoma avicola. This is a name for the alphanumeric GTDB genus CAG-145. This genus has been assigned by GTDB-Tk v1.3.0 working on GTDB Release 05-RS95 (Chaumeil et al., 2019; Parks et al., 2020) to the order Peptostreptococcales and to the family

4583

4584

4585

Description of Candidatus Fimisoma avicola sp. nov.

4586

Candidatus Fimisoma avicola (a.vi'co.la. L. fem. n. avis bird; L. suff. -cola inhabitant of; N.L. n.

4587 avicola inhabitant of birds)

4588

4589

A bacterial species identified by metagenomic analyses. This species includes all bacteria with genomes that show $\geq 95 \%$ average nucleotide identity (ANI) to the type genome, which has been assigned the MAG ID 11300 and which is available via NCBI BioSample SAMN15817197. This

4593

4594

4595 is a new name for the alphanumeric GTDB species sp900542565. The GC content of the type genome is $47.90 \%$ and the genome length is $2.0 \mathrm{Mbp}$.

\section{Description of Candidatus Fimivicinus gen. nov.}

4598

Candidatus Fimivicinus (Fim.i.vic.in'us. L. neut. n. fimum dung; L. masc. n. vicinus a neighbour; 4599

4600 N.L. masc. n. Fimivicinus a microbe associated with faeces)

4601

A bacterial genus identified by metagenomic analyses. The genus includes all bacteria with

4602

4603 genomes that show $\geq 60 \%$ average amino acid identity (AAI) to the type genome from the type species Fimivicinus intestinavium. This is a name for the alphanumeric GTDB genus UBA1691.

4604

4605 This genus has been assigned by GTDB-Tk v1.3.0 working on GTDB Release 05-RS95 (Chaumeil et al., 2019; Parks et al., 2020) to the order Oscillospirales and to the family

4606 Acutalibacteraceae.

4607

4608

Description of Candidatus Fimivicinus intestinavium sp. nov.

4609 Candidatus Fimivicinus intestinavium (in.tes.tin.a'vi.um. L. neut. n. intestinum gut; L. fem. n.

4610 avis bird; N.L. gen. n. intestinavium of the gut of birds)

4611

4612

4613

4614

4615

A bacterial species identified by metagenomic analyses. This species includes all bacteria with genomes that show $\geq 95 \%$ average nucleotide identity (ANI) to the type genome, which has been assigned the MAG ID 2526 and which is available via NCBI BioSample SAMN15817188. This is a new name for the alphanumeric GTDB species sp900552985. The GC content of the type

4616 4617 4618 genome is $55.20 \%$ and the genome length is $2.5 \mathrm{Mbp}$.

\section{Description of Candidatus Fimivivens gen. nov.}

4621

Candidatus Fimivivens (Fim.i.viv'ens. L. neut. n. fimum dung; N.L. pres. part. vivens living; N.L. 4622 fem. n. Fimivivens a microbe associated with faeces) 
4623 A bacterial genus identified by metagenomic analyses. The genus includes all bacteria with 4624 genomes that show $\geq 60 \%$ average amino acid identity (AAI) to the type genome from the type 4625 species Fimivivens faecavium. This is a name for the alphanumeric GTDB genus D5. This genus 4626 has been assigned by GTDB-Tk v1.3.0 working on GTDB Release 05-RS95 (Chaumeil et al., 4627 2019; Parks et al., 2020) to the order Oscillospirales and to the family Ruminococcaceae.

Description of Candidatus Fimivivens faecavium sp. nov.

4631 Candidatus Fimivivens faecavium (faec.a'vi.um. L. fem. n. faex, faecis excrement; L. fem. n.

4632

4633

4634 avis bird; N.L. gen. n. faecavium of bird faeces)

4635

4636

4637

4638

4639

4640

4641

4642

4643

4644

4645

4646

4647

4648

4649

4650

4651

4652

4653

4654

4655

4656

4657

A bacterial species identified by metagenomic analyses. This species includes all bacteria with genomes that show $\geq 95 \%$ average nucleotide identity (ANI) to the type genome, which has been assigned the MAG ID CHK195-35099 and which is available via NCBI BioSample

SAMN15817038. The GC content of the type genome is $58.86 \%$ and the genome length is 2.0 Mbp.

\section{Description of Candidatus Fimousia gen. nov.}

Candidatus Fimousia (Fim.ou'si.a. L. neut. n. fimum dung; Gr. fem. n. ousia an essence; N.L. fem. n. Fimousia a microbe associated with faeces)

A bacterial genus identified by metagenomic analyses. The genus includes all bacteria with genomes that show $\geq 60 \%$ average amino acid identity (AAI) to the type genome from the type species Fimousia stercorigallinarum. This is a name for the alphanumeric GTDB genus 992a. This genus has been assigned by GTDB-Tk v1.3.0 working on GTDB Release 05-RS95 (Chaumeil et al., 2019; Parks et al., 2020) to the order Lachnospirales and to the family Lachnospiraceae.

\section{Description of Candidatus Fimousia stercorigallinarum sp. nov.}

Candidatus Fimousia stercorigallinarum (ster.co.ri.gal.li.na'rum. L. neut. n. stercus dung; L. fem. n. gallina hen; N.L. gen. n. stercorigallinarum of hen faeces)

4658

A bacterial species identified by metagenomic analyses. This species includes all bacteria with genomes that show $\geq 95 \%$ average nucleotide identity (ANI) to the type genome, which has been 4659

4660

4661 assigned the MAG ID ChiSxjej3B15-1827 and which is available via NCBI BioSample

4662

4663

4664

4665 SAMN15817114. The GC content of the type genome is $41.52 \%$ and the genome length is 2.3 Mbp.

4666

Description of Candidatus Flavonifractor avicola sp. nov.

Candidatus Flavonifractor avicola (a.vi'co.la. L. fem. n. avis bird; L. suff. -cola inhabitant of; 4667 N.L. n. avicola inhabitant of birds) 
4668 A bacterial species identified by metagenomic analyses. This species includes all bacteria with 4669 genomes that show $\geq 95 \%$ average nucleotide identity (ANI) to the type genome, which has been 4670 assigned the MAG ID CHK178-4001 and which is available via NCBI BioSample

4671 SAMN15816843. This is a new name for the alphanumeric GTDB species sp002161085. The

4672 GC content of the type genome is $60.44 \%$ and the genome length is $2.5 \mathrm{Mbp}$.

4673

4674

4675

Description of Candidatus Flavonifractor avistercoris sp. nov.

4676 Candidatus Flavonifractor avistercoris (a.vi.ster'co.ris. L. fem. n. avis bird; L. neut. n. stercus

4677

4678

4679 dung; N.L. gen. n. avistercoris of bird faeces)

4680

A bacterial species identified by metagenomic analyses. This species includes all bacteria with

4681 genomes that show $\geq 95 \%$ average nucleotide identity (ANI) to the type genome, which has been assigned the MAG ID 6084 and which is available via NCBI BioSample SAMN15816821. This

4682 is a new name for the alphanumeric GTDB species sp002161215. The GC content of the type

4683

4684 genome is $65.16 \%$ and the genome length is $2.4 \mathrm{Mbp}$.

4685

4686

4687

Description of Candidatus Flavonifractor intestinigallinarum sp. nov.

Candidatus Flavonifractor intestinigallinarum (in.tes.ti.ni.gal.li.na'rum. L. neut. n. intestinum

4688

4689

4690 gut; L. fem. n. gallina hen; N.L. gen. n. intestinigallinarum of the gut of the hens)

4691

4692

4693

4694

4695

4696

4697

4698

4699

4700

4701

4702

4703

A bacterial species identified by metagenomic analyses. This species includes all bacteria with genomes that show $\geq 95 \%$ average nucleotide identity (ANI) to the type genome, which has been assigned the MAG ID CHK192-8294 and which is available via NCBI BioSample SAMN15816592. The GC content of the type genome is $61.61 \%$ and the genome length is 2.5 Mbp.

4704

4705

4706

4707

4708

Description of Candidatus Flavonifractor intestinipullorum sp. nov.

Candidatus Flavonifractor intestinipullorum (in.tes.ti.ni.pul.lo'rum. L. neut. n. intestinum gut; L. masc. n. pullus a young chicken; N.L. gen. n. intestinipullorum of the gut of young chickens)

A bacterial species identified by metagenomic analyses. This species includes all bacteria with genomes that show $\geq 95 \%$ average nucleotide identity (ANI) to the type genome, which has been assigned the MAG ID CHK189-11263 and which is available via NCBI BioSample SAMN15816594. The GC content of the type genome is $63.72 \%$ and the genome length is 2.2 Mbp.

4709

4710

4711

4712

Description of Candidatus Flavonifractor merdavium sp. nov.

Candidatus Flavonifractor merdavium (merd.a'vi.um. L. fem. n. merda faeces; L. fem. n. avis bird; N.L. gen. n. merdavium of bird faeces)

4713

A bacterial species identified by metagenomic analyses. This species includes all bacteria with genomes that show $\geq 95 \%$ average nucleotide identity (ANI) to the type genome, which has been 
4714 assigned the MAG ID 3313 and which is available via NCBI BioSample SAMN15816644. The

4715

4716

4717

4718

4719

4720

4721

4722

4723

4724

4725

4726

4727

4728

4729

4730

4731

4732

4733

4734

4735

4736

4737

4738

4739

4740

4741

4742

4743

4744

4745

4746

4747

4748

4749

4750

4751

4752

4753

4754

4755

4756

4757

4758

4759

$\mathrm{GC}$ content of the type genome is $62.46 \%$ and the genome length is $2.7 \mathrm{Mbp}$.

\section{Description of Candidatus Flavonifractor merdigallinarum sp. nov.} Candidatus Flavonifractor merdigallinarum (mer.di.gal.li.na'rum. L. fem. n. merda faeces; L. fem. n. gallina hen; N.L. gen. n. merdigallinarum of hen faeces)

A bacterial species identified by metagenomic analyses. This species includes all bacteria with genomes that show $\geq 95 \%$ average nucleotide identity (ANI) to the type genome, which has been assigned the MAG ID ChiBcec16-6824 and which is available via NCBI BioSample SAMN15816721. The GC content of the type genome is $61.09 \%$ and the genome length is 2.6 Mbp.

\section{Description of Candidatus Flavonifractor merdipullorum sp. nov.}

Candidatus Flavonifractor merdipullorum (mer.di.pul.lo'rum. L. fem. n. merda faeces; L. masc. n. pullus a young chicken; N.L. gen. n. merdipullorum of the faeces of young chickens)

A bacterial species identified by metagenomic analyses. This species includes all bacteria with genomes that show $\geq 95 \%$ average nucleotide identity (ANI) to the type genome, which has been assigned the MAG ID ChiGjej6B6-1540 and which is available via NCBI BioSample SAMN15816748. The GC content of the type genome is $61.06 \%$ and the genome length is 2.1 Mbp.

\section{Description of Candidatus Fournierella excrementavium sp. nov.}

Candidatus Fournierella excrementavium (ex.cre.ment.a'vi.um. L. neut. n. excrementum excrement; L. fem. n. avis bird; N.L. gen. n. excrementavium of bird excrement)

A bacterial species identified by metagenomic analyses. This species includes all bacteria with genomes that show $\geq 95 \%$ average nucleotide identity (ANI) to the type genome, which has been assigned the MAG ID ChiHcec27-1717 and which is available via NCBI BioSample SAMN15816881. This is a new name for the alphanumeric GTDB species sp004558145. The $\mathrm{GC}$ content of the type genome is $63.90 \%$ and the genome length is $2.4 \mathrm{Mbp}$.

\section{Description of Candidatus Fournierella excrementigallinarum sp. nov.}

Candidatus Fournierella excrementigallinarum (ex.cre.men.ti.gal.li.na'rum. L. neut. n. excrementum excrement; L. fem. n. gallina hen; N.L. gen. n. excrementigallinarum of hen excrement)

A bacterial species identified by metagenomic analyses. This species includes all bacteria with genomes that show $\geq 95 \%$ average nucleotide identity (ANI) to the type genome, which has been assigned the MAG ID 1136 and which is available via NCBI BioSample SAMN15816650. The $\mathrm{GC}$ content of the type genome is $64.27 \%$ and the genome length is $2.1 \mathrm{Mbp}$.

Peer) reviewing PDF | (2020:12:55980:1:1:NEW 13 Jan 2021) 
4760

4761

4762

4763

4764

4765

4766

4767

4768

4769

4770

4771

4772

4773

4774

4775

4776

4777

4778

4779

4780

4781

4782

4783

4784

4785

4786

4787

4788

4789

4790

4791

4792

4793

4794

4795

4796

4797

4798

4799

4800

4801

4802

4803

4804

\section{Description of Candidatus Fournierella merdavium sp. nov.}

Candidatus Fournierella merdavium (merd.a'vi.um. L. fem. n. merda faeces; L. fem. n. avis bird; N.L. gen. n. merdavium of bird faeces)

A bacterial species identified by metagenomic analyses. This species includes all bacteria with genomes that show $\geq 95 \%$ average nucleotide identity (ANI) to the type genome, which has been assigned the MAG ID ChiBcec4-1730 and which is available via NCBI BioSample

SAMN15816653. The GC content of the type genome is $64.33 \%$ and the genome length is 2.6 Mbp.

Description of Candidatus Fournierella merdigallinarum sp. nov.

Candidatus Fournierella merdigallinarum (mer.di.gal.li.na'rum. L. fem. n. merda faeces; L. fem. n. gallina hen; N.L. gen. n. merdigallinarum of hen faeces)

A bacterial species identified by metagenomic analyses. This species includes all bacteria with genomes that show $\geq 95 \%$ average nucleotide identity (ANI) to the type genome, which has been assigned the MAG ID 6296 and which is available via NCBI BioSample SAMN15816675. The $\mathrm{GC}$ content of the type genome is $65.05 \%$ and the genome length is $2.4 \mathrm{Mbp}$.

\section{Description of Candidatus Fournierella merdipullorum sp. nov.}

Candidatus Fournierella merdipullorum (mer.di.pul.lo'rum. L. fem. n. merda faeces; L. masc. n. pullus a young chicken; N.L. gen. n. merdipullorum of the faeces of young chickens)

A bacterial species identified by metagenomic analyses. This species includes all bacteria with genomes that show $\geq 95 \%$ average nucleotide identity (ANI) to the type genome, which has been assigned the MAG ID ChiGjej4B4-18154 and which is available via NCBI BioSample

SAMN15816693. The GC content of the type genome is $62.57 \%$ and the genome length is 2.5 Mbp.

\section{Description of Candidatus Fournierella pullicola sp. nov.}

Candidatus Fournierella pullicola (pul.li'co.la. L. masc. n. pullus a young chicken; L. suff. -cola inhabitant of; N.L. n. pullicola an inhabitant of young chickens)

A bacterial species identified by metagenomic analyses. This species includes all bacteria with genomes that show $\geq 95 \%$ average nucleotide identity (ANI) to the type genome, which has been assigned the MAG ID 2239 and which is available via NCBI BioSample SAMN15816745. The GC content of the type genome is $62.57 \%$ and the genome length is $2.4 \mathrm{Mbp}$.

\section{Description of Candidatus Fournierella pullistercoris sp. nov.}

Peer] reviewing PDF | (2020:12:55980:1:1:NEW 13 Jan 2021) 
4805 Candidatus Fournierella pullistercoris (pul.li.ster'co.ris. L. masc. n. pullus a young chicken; L.

4806

4807

4808

4809

4810

4811

4812

4813

4814

4815

4816

4817

4818

4819

4820

4821

4822

4823

4824

4825

4826

4827

4828

4829

4830

4831

4832

4833

4834

4835

4836

4837

4838

4839

4840

4841

4842

4843

4844

4845

4846

4847

4848

4849

4850

neut. n. stercus dung; N.L. gen. n. pullistercoris of young chicken faeces)

A bacterial species identified by metagenomic analyses. This species includes all bacteria with genomes that show $\geq 95 \%$ average nucleotide identity (ANI) to the type genome, which has been assigned the MAG ID B5-2728 and which is available via NCBI BioSample SAMN15816762. The $\mathrm{GC}$ content of the type genome is $52.45 \%$ and the genome length is $1.7 \mathrm{Mbp}$.

\section{Description of Candidatus Fusicatenibacter intestinigallinarum sp. nov.}

Candidatus Fusicatenibacter intestinigallinarum (in.tes.ti.ni.gal.li.na'rum. L. neut. n. intestinum gut; L. fem. n. gallina hen; N.L. gen. n. intestinigallinarum of the gut of the hens)

A bacterial species identified by metagenomic analyses. This species includes all bacteria with genomes that show $\geq 95 \%$ average nucleotide identity (ANI) to the type genome, which has been assigned the MAG ID CHK185-5351 and which is available via NCBI BioSample SAMN15816585. The GC content of the type genome is $51.22 \%$ and the genome length is 2.9 Mbp.

\section{Description of Candidatus Fusicatenibacter intestinipullorum sp. nov.}

Candidatus Fusicatenibacter intestinipullorum (in.tes.ti.ni.pul.lo'rum. L. neut. n. intestinum gut; L. masc. n. pullus a young chicken; N.L. gen. n. intestinipullorum of the gut of young chickens)

A bacterial species identified by metagenomic analyses. This species includes all bacteria with genomes that show $\geq 95 \%$ average nucleotide identity (ANI) to the type genome, which has been assigned the MAG ID ChiBcec11-5794 and which is available via NCBI BioSample SAMN15816833. This is a new name for the alphanumeric GTDB species sp900543115. The $\mathrm{GC}$ content of the type genome is $49.70 \%$ and the genome length is $2.6 \mathrm{Mbp}$.

\section{Description of Candidatus Fusicatenibacter merdavium sp. nov.}

Candidatus Fusicatenibacter merdavium (merd.a'vi.um. L. fem. n. merda faeces; L. fem. n. avis bird; N.L. gen. n. merdavium of bird faeces)

A bacterial species identified by metagenomic analyses. This species includes all bacteria with genomes that show $\geq 95 \%$ average nucleotide identity (ANI) to the type genome, which has been assigned the MAG ID CHK183-1962 and which is available via NCBI BioSample SAMN15816614. The GC content of the type genome is $51.02 \%$ and the genome length is 2.7 Mbp.

\section{Description of Candidatus Fusobacterium pullicola sp. nov.}

Candidatus Fusobacterium pullicola (pul.li'co.la. L. masc. n. pullus a young chicken; L. suff. cola inhabitant of; N.L. n. pullicola inhabitant of a young chicken)

PeerJ reviewing PDF | (2020:12:55980:1:1:NEW 13 Jan 2021) 
4851 A bacterial species identified by metagenomic analyses. This species includes all bacteria with

4852

4853

4854

4855

4856

4857

4858

4859

4860

4861

4862

4863

4864

4865

4866

4867

4868

4869

4870

4871

4872

4873

4874

4875

4876

4877

4878

4879

4880

4881

4882

4883

4884

4885

4886

4887

4888

4889

4890

4891

4892

4893

4894

4895 genomes that show $\geq 95 \%$ average nucleotide identity (ANI) to the type genome, which has been assigned the MAG ID A6-441 and which is available via NCBI BioSample SAMN15816927. This is a new name for the alphanumeric GTDB species sp900549465. Although GTDB has assigned this species to the genus it calls Fusobacterium_A, this genus designation cannot be incorporated into a well-formed binomial, so in naming this species, we have used the current validly published name for the genus. The GC content of the type genome is $29.86 \%$ and the genome length is $1.8 \mathrm{Mbp}$.

\section{Description of Candidatus Gallacutalibacter gen. nov.}

Candidatus Gallacutalibacter (Gall.a.cu.ta.li.bac'ter. L. masc. n. gallus chicken; N.L. masc. n. Acutalibacter a genus name; N.L. masc. n. Gallacutalibacter a genus related to the genus Acutalibacter but distinct from it and found in poultry)

A bacterial genus identified by metagenomic analyses. The genus includes all bacteria with genomes that show $\geq 60 \%$ average amino acid identity (AAI) to the type genome from the type species Gallacutalibacter pullicola. This genus was identified but not named by Glendinning et al. This genus has been assigned by GTDB-Tk v1.3.0 working on GTDB Release 05-RS95 (Chaumeil et al., 2019; Parks et al., 2020) to the order Oscillospirales and to the family Acutalibacteraceae.

\section{Description of Candidatus Gallacutalibacter pullicola sp. nov.}

Candidatus Gallacutalibacter pullicola (pul.li'co.la. L. masc. n. pullus a young chicken; L. suff. cola inhabitant of; N.L. n. pullicola an inhabitant of young chickens)

A bacterial species identified by metagenomic analyses. This species includes all bacteria with genomes that show $\geq 95 \%$ average nucleotide identity (ANI) to the type genome, which has been assigned the MAG ID ChiSjej1B19-7085 and which is available via NCBI BioSample

SAMN15816935. The GC content of the type genome is $56.02 \%$ and the genome length is 2.5 Mbp.

\section{Description of Candidatus Gallacutalibacter pullistercoris sp. nov.}

Candidatus Gallacutalibacter pullistercoris (pul.li.ster'co.ris. L. masc. n. pullus a young chicken; L. neut. n. stercus dung; N.L. gen. n. pullistercoris of young chicken faeces)

A bacterial species identified by metagenomic analyses. This species includes all bacteria with genomes that show $\geq 95 \%$ average nucleotide identity (ANI) to the type genome, which has been assigned the MAG ID 13869 and which is available via NCBI BioSample SAMN15816961. The $\mathrm{GC}$ content of the type genome is $51.21 \%$ and the genome length is $2.4 \mathrm{Mbp}$.

\section{Description of Candidatus Gallacutalibacter stercoravium sp. nov.}

Peer] reviewing PDF | (2020:12:55980:1:1:NEW 13 Jan 2021) 
4896 Candidatus Gallacutalibacter stercoravium (ster.cor.a'vi.um. L. neut. n. stercus dung; L. fem. n. 4897 avis bird; N.L. gen. n. stercoravium of bird faeces)

4898

4899

4900

4901

4902

4903

4904

4905

4906

4907

4908

4909

4910

4911

4912

4913

4914

4915

4916

4917

4918

4919

4920

4921

4922

4923

4924

4925

4926

4927

4928

4929

4930

4931

4932

4933

4934

4935

4936

4937

4938

4939

4940

4941

A bacterial species identified by metagenomic analyses. This species includes all bacteria with genomes that show $\geq 95 \%$ average nucleotide identity (ANI) to the type genome, which has been assigned the MAG ID CHK176-13069 and which is available via NCBI BioSample SAMN15816939. The GC content of the type genome is $51.38 \%$ and the genome length is 2.7 Mbp.

\section{Description of Candidatus Gallibacteroides gen. nov.}

Candidatus Gallibacteroides (Gal.li.bac.te.ro'i.des. L. masc. n. gallus chicken; N.L. masc. n. Bacteroides a genus name; N.L. masc. n. Gallibacteroides a genus related to the genus Bacteroides but distinct from it and found in poultry)

A bacterial genus identified by metagenomic analyses. The genus includes all bacteria with genomes that show $\geq 60 \%$ average amino acid identity (AAI) to the type genome from the type species Gallibacteroides avistercoris. This genus has been assigned by GTDB-Tk v1.3.0 working on GTDB Release 05-RS95 (Chaumeil et al., 2019; Parks et al., 2020) to the order Bacteroidales and to the family Barnesiellaceae.

\section{Description of Candidatus Gallibacteroides avistercoris sp. nov.}

Candidatus Gallibacteroides avistercoris (a.vi.ster'co.ris. L. fem. n. avis bird; L. neut. n. stercus dung; N.L. gen. n. avistercoris of bird faeces)

A bacterial species identified by metagenomic analyses. This species includes all bacteria with genomes that show $\geq 95 \%$ average nucleotide identity (ANI) to the type genome, which has been assigned the MAG ID CHK158-818 and which is available via NCBI BioSample

SAMN15816984. The GC content of the type genome is $46.12 \%$ and the genome length is 2.3 Mbp.

\section{Description of Candidatus Galligastranaerophilus gen. nov.}

Candidatus Galligastranaerophilus (Gal.li.gastr.an.a.e.ro'phi.lus. L. masc. n. gallus chicken; N.L. masc. n. Gastranaerophilus a genus name; N.L. masc. n. Galligastranaerophilus a genus related to the genus Gastranaerophilus but distinct from it and found in poultry)

A bacterial genus identified by metagenomic analyses. The genus includes all bacteria with genomes that show $\geq 60 \%$ average amino acid identity (AAI) to the type genome from the type species Galligastranaerophilus faecipullorum. This genus has been assigned by GTDB-Tk v1.3.0 working on GTDB Release 05-RS95 (Chaumeil et al., 2019; Parks et al., 2020) to the order Gastranaerophilales and to the family Gastranaerophilaceae.

\section{Description of Candidatus Galligastranaerophilus faecipullorum sp. nov.}


4942 Candidatus Galligastranaerophilus faecipullorum (fae.ci.pul.lo'rum. L. fem. n. faex, faecis

4943 excrement; L. masc. n. pullus a young chicken; N.L. gen. n. faecipullorum of young chicken

4944 faeces)

4945

4946

4947

4948

4949

4950

4951

4952

4953

4954

4955

4956

4957

4958

4959

4960

4961

4962

4963

4964

4965

4966

4967

4968

4969

4970

4971

4972

4973

4974

4975

4976

4977

4978

4979

4980

4981

4982

4983

4984

4985

4986

\section{Description of Candidatus Galligastranaerophilus gallistercoris sp. nov.}

Candidatus Galligastranaerophilus gallistercoris (gal.li.ster'co.ris. L. masc. n gallus chicken; L. neut. n. stercus dung; N.L. gen. n. gallistercoris of chicken faeces)

A bacterial species identified by metagenomic analyses. This species includes all bacteria with genomes that show $\geq 95 \%$ average nucleotide identity (ANI) to the type genome, which has been assigned the MAG ID CHK123-4750 and which is available via NCBI BioSample SAMN15816963. The GC content of the type genome is $35.45 \%$ and the genome length is 1.8 Mbp.

\section{Description of Candidatus Galligastranaerophilus intestinavium sp. nov.}

Candidatus Galligastranaerophilus intestinavium (in.tes.tin.a'vi.um. L. neut. n. intestinum gut; L. fem. n. avis bird; N.L. gen. n. intestinavium of the gut of birds)

A bacterial species identified by metagenomic analyses. This species includes all bacteria with genomes that show $\geq 95 \%$ average nucleotide identity (ANI) to the type genome, which has been assigned the MAG ID CHK152-2871 and which is available via NCBI BioSample SAMN15816967. The GC content of the type genome is $35.80 \%$ and the genome length is 1.6 Mbp.

\section{Description of Candidatus Galligastranaerophilus intestinigallinarum sp. nov.} Candidatus Galligastranaerophilus intestinigallinarum (in.tes.ti.ni.gal.li.na'rum. L. neut. n. intestinum gut; L. fem. n. gallina hen; N.L. gen. n. intestinigallinarum of the gut of the hens)

A bacterial species identified by metagenomic analyses. This species includes all bacteria with genomes that show $\geq 95 \%$ average nucleotide identity (ANI) to the type genome, which has been assigned the MAG ID CHK123-3438 and which is available via NCBI BioSample SAMN15816973. The GC content of the type genome is $32.50 \%$ and the genome length is 1.7 Mbp.

\section{Description of Candidatus Gallilactobacillus gen. nov.}


4987 Candidatus Gallilactobacillus (Gal.li.lac.to.ba.cil'lus. L. masc. n. gallus chicken; N.L. masc. n. 4988 Lactobacillus a genus name; N.L. masc. n. Gallilactobacillus a genus related to the genus

4989 Lactobacillus but distinct from it and found in poultry)

4990

4991

A bacterial genus identified by metagenomic analyses. The genus includes all bacteria with

4992

4993

4994 genomes that show $\geq 60 \%$ average amino acid identity (AAI) to the type genome from the type species Gallilactobacillus intestinavium. This genus has been assigned by GTDB-Tk v1.3.0 working on GTDB Release 05-RS95 (Chaumeil et al., 2019; Parks et al., 2020) to the order

4995 Lactobacillales and to the family Lactobacillaceae.

4996

4997

4998

Description of Candidatus Gallilactobacillus intestinavium sp. nov.

4999 Candidatus Gallilactobacillus intestinavium (in.tes.tin.a'vi.um. L. neut. n. intestinum gut; L. fem.

5000

5001

5002

n. avis bird; N.L. gen. n. intestinavium of the gut of birds)

5003

A bacterial species identified by metagenomic analyses. This species includes all bacteria with

5004 genomes that show $\geq 95 \%$ average nucleotide identity (ANI) to the type genome, which has been assigned the MAG ID C6-149 and which is available via NCBI BioSample SAMN15816970.

5005 The GC content of the type genome is $29.69 \%$ and the genome length is $1.2 \mathrm{Mbp}$.

5006

5007

5008

Description of Candidatus Gallimonas gallistercoris sp. nov.

5009 Candidatus Gallimonas gallistercoris (gal.li.ster'co.ris. L. masc. n gallus chicken; L. neut. n.

5010 stercus dung; N.L. gen. n. gallistercoris of chicken faeces)

5011

5012

A bacterial species identified by metagenomic analyses. This species includes all bacteria with

5013

5014 genomes that show $\geq 95 \%$ average nucleotide identity (ANI) to the type genome, which has been assigned the MAG ID CHK156-179 and which is available via NCBI BioSample

5015

5016

5017

SAMN15816677. The GC content of the type genome is $58.55 \%$ and the genome length is 1.6 Mbp.

5018

5019

Description of Candidatus Gallimonas intestinavium sp. nov.

5020

Candidatus Gallimonas intestinavium (in.tes.tin.a'vi.um. L. neut. n. intestinum gut; L. fem. $n$.

5021

5022

5023 avis bird; N.L. gen. n. intestinavium of the gut of birds)

5024

5025

5026

5027

5028

5029

5030

5031

A bacterial species identified by metagenomic analyses. This species includes all bacteria with genomes that show $\geq 95 \%$ average nucleotide identity (ANI) to the type genome, which has been assigned the MAG ID ChiW7-2402 and which is available via NCBI BioSample

SAMN15816844. This is a new name for the alphanumeric GTDB species sp003343805. The $\mathrm{GC}$ content of the type genome is $58.63 \%$ and the genome length is $1.8 \mathrm{Mbp}$.

\section{Description of Candidatus Gallimonas intestinigallinarum sp. nov.}

Candidatus Gallimonas intestinigallinarum (in.tes.ti.ni.gal.li.na'rum. L. neut. n. intestinum gut;

5032

L. fem. n. gallina hen; N.L. gen. n. intestinigallinarum of the gut of the hens)

Peer] reviewing PDF | (2020:12:55980:1:1:NEW 13 Jan 2021) 
5033

5034

5035

5036

5037

5038

5039

5040

5041

5042

5043

5044

5045

5046

5047

5048

5049

5050

5051

5052

5053

5054

5055

5056

5057

5058

5059

5060

5061

5062

5063

5064

5065

5066

5067

5068

5069

5070

5071

5072

5073

5074

5075

5076

5077

5078
A bacterial species identified by metagenomic analyses. This species includes all bacteria with genomes that show $\geq 95 \%$ average nucleotide identity (ANI) to the type genome, which has been assigned the MAG ID CHK33-5263 and which is available via NCBI BioSample

SAMN15816692. The GC content of the type genome is $57.82 \%$ and the genome length is 1.6 Mbp.

\section{Description of Candidatus Gallipaludibacter gen. nov.}

Candidatus Gallipaludibacter (Gal.li.pa.lu.di.bac'ter. L. masc. n. gallus chicken; N.L. masc. n. Paludibacter a genus name; N.L. masc. n. Gallipaludibacter a genus related to the genus Paludibacter but distinct from it and found in poultry)

A bacterial genus identified by metagenomic analyses. The genus includes all bacteria with genomes that show $\geq 60 \%$ average amino acid identity (AAI) to the type genome from the type species Gallipaludibacter merdavium. This genus has been assigned by GTDB-Tk v1.3.0 working on GTDB Release 05-RS95 (Chaumeil et al., 2019; Parks et al., 2020) to the order Bacteroidales and to the family Paludibacteraceae.

\section{Description of Candidatus Gallipaludibacter merdavium sp. nov.}

Candidatus Gallipaludibacter merdavium (merd.a'vi.um. L. fem. n. merda faeces; L. fem. n. avis bird; N.L. gen. n. merdavium of bird faeces)

A bacterial species identified by metagenomic analyses. This species includes all bacteria with genomes that show $\geq 95 \%$ average nucleotide identity (ANI) to the type genome, which has been assigned the MAG ID G3-3990 and which is available via NCBI BioSample SAMN15816954. The GC content of the type genome is $41.91 \%$ and the genome length is $2.9 \mathrm{Mbp}$.

\section{Description of Candidatus Gallitreponema gen. nov.}

Candidatus Gallitreponema (Gal.li.tre.po.ne'ma. L. masc. n. gallus chicken; N.L. neut. n. Treponema a genus name; N.L. neut. n. Gallitreponema a genus related to the genus Treponema but distinct from it and found in poultry)

A bacterial genus identified by metagenomic analyses. The genus includes all bacteria with genomes that show $\geq 60 \%$ average amino acid identity (AAI) to the type genome from the type species Gallitreponema excrementavium. This genus has been assigned by GTDB-Tk v1.3.0 working on GTDB Release 05-RS95 (Chaumeil et al., 2019; Parks et al., 2020) to the order Treponematales and to the family Treponemataceae.

\section{Description of Candidatus Gallitreponema excrementavium sp. nov.}

Candidatus Gallitreponema excrementavium (ex.cre.ment.a'vi.um. L. neut. n. excrementum excrement; L. fem. n. avis bird; N.L. gen. n. excrementavium of bird excrement)

Peer) reviewing PDF | (2020:12:55980:1:1:NEW 13 Jan 2021) 
5079 A bacterial species identified by metagenomic analyses. This species includes all bacteria with

5080

5081

5082

5083

5084

5085

5086

5087

5088

5089

5090

5091

5092

5093

5094

5095

5096

5097

5098

5099

5100

5101

5102

5103

5104

5105

5106

5107

5108

5109

5110

5111

5112

5113

5114

5115

5116

5117

5118

5119

5120

5121

5122

5123

5124 genomes that show $\geq 95 \%$ average nucleotide identity (ANI) to the type genome, which has been assigned the MAG ID 10532 and which is available via NCBI BioSample SAMN15816962. The $\mathrm{GC}$ content of the type genome is $40.13 \%$ and the genome length is $2.4 \mathrm{Mbp}$.

\section{Description of Candidatus Galloscillospira gen. nov.}

Candidatus Galloscillospira (Gall.os.cil.lo.spi'ra. L. masc. n. gallus chicken; N.L. fem. n. Oscillospira a genus name; N.L. fem. n. Galloscillospira. a genus related to the genus Oscillospira but distinct from it and found in poultry)

A bacterial genus identified by metagenomic analyses. The genus includes all bacteria with genomes that show $\geq 60 \%$ average amino acid identity (AAI) to the type genome from the type species Galloscillospira excrementipullorum. This genus belongs to the new family Candidatus Galloscillospiraceae.

\section{Description of Candidatus Galloscillospira excrementavium sp. nov.}

Candidatus Galloscillospira excrementavium (ex.cre.ment.a'vi.um. L. neut. n. excrementum excrement; L. fem. n. avis bird; N.L. gen. n. excrementavium of bird excrement)

A bacterial species identified by metagenomic analyses. This species includes all bacteria with genomes that show $\geq 95 \%$ average nucleotide identity (ANI) to the type genome, which has been assigned the MAG ID ChiSjej1B19-13426 and which is available via NCBI BioSample SAMN15816937. The GC content of the type genome is $65.18 \%$ and the genome length is 2.1 Mbp.

\section{Description of Candidatus Galloscillospira excrementipullorum sp. nov.} Candidatus Galloscillospira excrementipullorum (ex.cre.men.ti.pul.lo'rum. L. neut. n. excrementum excrement; L. masc. n. pullus a young chicken; N.L. gen. n. excrementipullorum of young chicken excrement)

A bacterial species identified by metagenomic analyses. This species includes all bacteria with genomes that show $\geq 95 \%$ average nucleotide identity (ANI) to the type genome, which has been assigned the MAG ID ChiHjej8B7-10251 and which is available via NCBI BioSample SAMN15816946. The GC content of the type genome is $60.78 \%$ and the genome length is 1.6 Mbp.

\section{Description of Candidatus Galloscillospira stercoripullorum sp. nov.}

Candidatus Galloscillospira stercoripullorum (ster.co.ri.pul.lo'rum. L. neut. n. stercus dung; L. masc. n. pullus a young chicken; N.L. gen. n. stercoripullorum of the faceces of young chickens)

A bacterial species identified by metagenomic analyses. This species includes all bacteria with genomes that show $\geq 95 \%$ average nucleotide identity (ANI) to the type genome, which has been 
5125 assigned the MAG ID CHK33-6455 and which is available via NCBI BioSample

5126 SAMN15816975. The GC content of the type genome is $62.96 \%$ and the genome length is 1.9

5127 Mbp.

5128

5129

5130

5131

5132

5133

5134

5135

5136

5137

5138

5139

5140

5141

5142

5143

5144

5145

5146

5147

5148

5149

5150

\section{Description of Candidatus Galloscillospiraceae fam. nov.}

Candidatus Galloscillospiraceae (Gall.os.cil.lo.spi.ra.ce'ae. N.L. fem. n. Galloscillospira. type genus of the family genus; N.L. suff. -ceae to denote a family; N.L. fem. pl. n.

Galloscillospiraceae, the family of the genus Galloscillospira)

A bacterial family identified by metagenomic analyses. This family has been defined by the absence of a family assignment for the type species when GTDB-Tk v1.3.0 is applied to GTDB Release 05-RS95 (Chaumeil et al., 2019; Parks et al., 2020). GTDB assigns the type species and thus the family to the order Oscillospirales.

\section{Description of Candidatus Gemmiger avicola sp. nov.}

Candidatus Gemmiger avicola (a.vi'co.la. L. fem. n. avis bird; L. suff. -cola inhabitant of; N.L. n. avicola inhabitant of birds)

A bacterial species identified by metagenomic analyses. This species includes all bacteria with genomes that show $\geq 95 \%$ average nucleotide identity (ANI) to the type genome, which has been assigned the MAG ID ChiBcec8-13705 and which is available via NCBI BioSample SAMN15816825. This is a new name for the alphanumeric GTDB species sp900548355. The GC content of the type genome is $61.69 \%$ and the genome length is $2.3 \mathrm{Mbp}$.

5151

5152

5153

Description of Candidatus Gemmiger avistercoris sp. nov.

Candidatus Gemmiger avistercoris (a.vi.ster'co.ris. L. fem. n. avis bird; L. neut. n. stercus dung;

5154

5155

5156

5157

5158

5159 N.L. gen. n. avistercoris of bird faeces)

A bacterial species identified by metagenomic analyses. This species includes all bacteria with genomes that show $\geq 95 \%$ average nucleotide identity (ANI) to the type genome, which has been assigned the MAG ID CHK188-11489 and which is available via NCBI BioSample

5160 Mbp.

5161

5162

5163

5164

5165

5166

5167

5168

5169

\section{Description of Candidatus Gemmiger avium sp. nov.}

Candidatus Gemmiger avium (a'vi.um. L. fem. pl. n. avium of birds)

A bacterial species identified by metagenomic analyses. This species includes all bacteria with genomes that show $\geq 95 \%$ average nucleotide identity (ANI) to the type genome, which has been assigned the MAG ID ChiGjej4B4-15321 and which is available via NCBI BioSample

5170 SAMN15816926. This is a new name for the alphanumeric GTDB species sp002160955. Although GTDB has assigned this species to the genus it calls Gemmiger_A, this genus 
5171 designation cannot be incorporated into a well-formed binomial, so in naming this species, we 5172 have used the current validly published name for the genus. The GC content of the type genome 5173 is $62.71 \%$ and the genome length is $2.6 \mathrm{Mbp}$.

5174

5175

5176

5177

\section{Description of Candidatus Gemmiger excrementavium sp. nov.} Candidatus Gemmiger excrementavium (ex.cre.ment.a'vi.um. L. neut. n. excrementum

5178

5179

5180

5181

5182

5183

5184

5185

5186

5187

5188

5189 excrement; L. fem. n. avis bird; N.L. gen. n. excrementavium of bird excrement)

A bacterial species identified by metagenomic analyses. This species includes all bacteria with genomes that show $\geq 95 \%$ average nucleotide identity (ANI) to the type genome, which has been assigned the MAG ID 3436 and which is available via NCBI BioSample SAMN15816690. The $\mathrm{GC}$ content of the type genome is $60.89 \%$ and the genome length is $2.5 \mathrm{Mbp}$.

\section{Description of Candidatus Gemmiger excrementigallinarum sp. nov.}

Candidatus Gemmiger excrementigallinarum (ex.cre.men.ti.gal.li.na'rum. L. neut. n. excrementum excrement; L. fem. n. avis bird; N.L. gen. n. excrementigallinarum of hen excrement)

5190

5191

5192

5193

5194

5195

5196

5197

5198

5199

5200

5201

5202

5203

5204

5205

5206

5207

5208

5209

5210

5211

5212

5213

5214

5215

5216

A bacterial species identified by metagenomic analyses. This species includes all bacteria with genomes that show $\geq 95 \%$ average nucleotide identity (ANI) to the type genome, which has been assigned the MAG ID ChiSxjej1B13-11774 and which is available via NCBI BioSample SAMN15816691. The GC content of the type genome is $58.73 \%$ and the genome length is 2.4 Mbp.

\section{Description of Candidatus Gemmiger excrementipullorum sp. nov.}

Candidatus Gemmiger excrementipullorum (ex.cre.men.ti.pul.lo'rum. L. neut. n. excrementum excrement; L. masc. n. pullus a young chicken; N.L. gen. n. excrementipullorum of young chicken excrement)

A bacterial species identified by metagenomic analyses. This species includes all bacteria with genomes that show $\geq 95 \%$ average nucleotide identity (ANI) to the type genome, which has been assigned the MAG ID ChiHecec2B26-7398 and which is available via NCBI BioSample SAMN15816726. The GC content of the type genome is $64.11 \%$ and the genome length is 2.1 Mbp.

\section{Description of Candidatus Gemmiger faecavium sp. nov.}

Candidatus Gemmiger faecavium (faec.a'vi.um. L. fem. n. faex, faecis excrement; L. fem. n. avis bird; N.L. gen. n. faecavium of bird faeces)

A bacterial species identified by metagenomic analyses. This species includes all bacteria with genomes that show $\geq 95 \%$ average nucleotide identity (ANI) to the type genome, which has been assigned the MAG ID ChiSxjej1B13-1558 and which is available via NCBI BioSample 
5217 SAMN15816740. The GC content of the type genome is $60.04 \%$ and the genome length is 2.5

$5218 \mathrm{Mbp}$.

5219

5220

5221

5222

Description of Candidatus Gemmiger faecigallinarum sp. nov.

Candidatus Gemmiger faecigallinarum (fae.ci.gal.li.na'rum. L. fem. n. faex, faecis excrement; L.

5223

5224

5225

fem. n. gallina hen; N.L. gen. n. faecigallinarum of hen faeces)

A bacterial species identified by metagenomic analyses. This species includes all bacteria with genomes that show $\geq 95 \%$ average nucleotide identity (ANI) to the type genome, which has been assigned the MAG ID 14795 and which is available via NCBI BioSample SAMN15816795. The $\mathrm{GC}$ content of the type genome is $63.90 \%$ and the genome length is $2.6 \mathrm{Mbp}$.

5229

5230

5231

\section{Description of Candidatus Gemmiger stercoravium sp. nov.}

5232

Candidatus Gemmiger stercoravium (ster.cor.a'vi.um. L. neut. n. stercus dung; L. fem. n. avis

5233

5234

5235

bird; N.L. gen. n. stercoravium of bird faeces)

A bacterial species identified by metagenomic analyses. This species includes all bacteria with genomes that show $\geq 95 \%$ average nucleotide identity (ANI) to the type genome, which has been assigned the MAG ID ChiBcolR8-2160 and which is available via NCBI BioSample

5237

5238

5239 SAMN15816579. The GC content of the type genome is $65.46 \%$ and the genome length is 2.5 Mbp.

5240

5241

5242

Description of Candidatus Gemmiger stercorigallinarum sp. nov.

5243

5244

5245

5246

Candidatus Gemmiger stercorigallinarum (ster.co.ri.gal.li.na'rum. L. neut. n. stercus dung; L. fem. n. gallina hen; N.L. gen. n. stercorigallinarum of hen faeces)

5247

A bacterial species identified by metagenomic analyses. This species includes all bacteria with genomes that show $\geq 95 \%$ average nucleotide identity (ANI) to the type genome, which has been assigned the MAG ID CHK183-27628 and which is available via NCBI BioSample SAMN15816586. The GC content of the type genome is $64.08 \%$ and the genome length is 2.5

5250

5251

5252

5253

5254

5255

5256 Mbp.

\section{Description of Candidatus Gemmiger stercoripullorum sp. nov.}

Candidatus Gemmiger stercoripullorum (ster.co.ri.pul.lo'rum. L. neut. n. stercus dung; L. masc. n. pullus a young chicken; N.L. gen. n. stercoripullorum of the faceces of young chickens)

A bacterial species identified by metagenomic analyses. This species includes all bacteria with genomes that show $\geq 95 \%$ average nucleotide identity (ANI) to the type genome, which has been assigned the MAG ID ChiSjej4B22-15101 and which is available via NCBI BioSample

5261

5262 SAMN15816589. The GC content of the type genome is $64.37 \%$ and the genome length is 2.2 Mbp. 
Description of Candidatus Gordonibacter avicola sp. nov.

5265 Candidatus Gordonibacter avicola (a.vi'co.la. L. fem. n. avis bird; L. suff. -cola inhabitant of;

N.L. n. avicola inhabitant of birds)

5269

A bacterial species identified by metagenomic analyses. This species includes all bacteria with genomes that show $\geq 95 \%$ average nucleotide identity (ANI) to the type genome, which has been

5270

5271

5272 assigned the MAG ID ChiSxjej6B18-1472 and which is available via NCBI BioSample SAMN15816757. The GC content of the type genome is $59.91 \%$ and the genome length is 3.1 Mbp.

5273

5274

5275

Description of Candidatus Halomonas stercoripullorum sp. nov.

5276

5277

Candidatus Halomonas stercoripullorum (ster.co.ri.pul.lo'rum. L. neut. n. stercus dung; L. masc. n. pullus a young chicken; N.L. gen. n. stercoripullorum of the faceces of young chickens)

5278

5279

A bacterial species identified by metagenomic analyses. This species includes all bacteria with 5280 genomes that show $\geq 95 \%$ average nucleotide identity (ANI) to the type genome, which has been assigned the MAG ID 1193 and which is available via NCBI BioSample SAMN15816734. The $\mathrm{GC}$ content of the type genome is $59.74 \%$ and the genome length is $2.1 \mathrm{Mbp}$.

Description of Candidatus Helicobacter avicola sp. nov.

5286 Candidatus Helicobacter avicola (a.vi'co.la. L. fem. n. avis bird; L. suff. -cola inhabitant of; N.L.

A bacterial species identified by metagenomic analyses. This species includes all bacteria with genomes that show $\geq 95 \%$ average nucleotide identity (ANI) to the type genome, which has been assigned the MAG ID 14449 and which is available via NCBI BioSample SAMN15816913. Although GTDB has assigned this species to the genus it calls Helicobacter_F, this genus designation cannot be incorporated into a well-formed binomial, so in naming this species, we have used the current validly published name for the genus. The GC content of the type genome is $42.81 \%$ and the genome length is $1.7 \mathrm{Mbp}$.

\section{Description of Candidatus Helicobacter avistercoris sp. nov.}

5299 Candidatus Helicobacter avistercoris (a.vi.ster'co.ris. L. fem. n. avis bird; L. neut. n. stercus dung; N.L. gen. n. avistercoris of bird faeces)

A bacterial species identified by metagenomic analyses. This species includes all bacteria with genomes that show $\geq 95 \%$ average nucleotide identity (ANI) to the type genome, which has been assigned the MAG ID CHK158-8274 and which is available via NCBI BioSample SAMN15816903. Although GTDB has assigned this species to the genus it calls Helicobacter_G , this genus designation cannot be incorporated into a well-formed binomial, so in naming this 5308 species, we have used the current validly published name for the genus. The GC content of the type genome is $38.34 \%$ and the genome length is $1.4 \mathrm{Mbp}$. 
Description of Candidatus Hungatella pullicola sp. nov.

5312 Candidatus Hungatella pullicola (pul.li'co.la. L. masc. n. pullus a young chicken; L. suff. -cola

5313 inhabitant of; N.L. n. pullicola an inhabitant of young chickens)

5314

5315

5316

5317

A bacterial species identified by metagenomic analyses. This species includes all bacteria with genomes that show $\geq 95 \%$ average nucleotide identity (ANI) to the type genome, which has been assigned the MAG ID CHK186-17716 and which is available via NCBI BioSample

5318

5319 SAMN15816620. The GC content of the type genome is $44.57 \%$ and the genome length is 2.9 Mbp.

5320

5321

5322

5323

Description of Candidatus Ignatzschineria merdigallinarum sp. nov. Candidatus Ignatzschineria merdigallinarum (mer.di.gal.li.na'rum. L. fem. n. merda faeces; L.

5324

5325

5326

5327

5328

5329 fem. n. gallina hen; N.L. gen. n. merdigallinarum of hen faeces)

A bacterial species identified by metagenomic analyses. This species includes all bacteria with genomes that show $\geq 95 \%$ average nucleotide identity (ANI) to the type genome, which has been assigned the MAG ID CHK160-9182 and which is available via NCBI BioSample SAMN15816769. The GC content of the type genome is $39.66 \%$ and the genome length is 2.3

5330

5331

5332

5333 Mbp.

\section{Description of Candidatus Intestinimonas merdavium sp. nov.}

Candidatus Intestinimonas merdavium (merd.a'vi.um. L. fem. n. merda faeces; L. fem. n. avis

5335

5336

5337 bird; N.L. gen. n. merdavium of bird faeces)

5338

A bacterial species identified by metagenomic analyses. This species includes all bacteria with genomes that show $\geq 95 \%$ average nucleotide identity (ANI) to the type genome, which has been

5339

5340

5341 assigned the MAG ID CHK33-7979 and which is available via NCBI BioSample

SAMN15816706. The GC content of the type genome is $61.44 \%$ and the genome length is 2.4 Mbp.

5342

5343

\section{Description of Candidatus Intestinimonas pullistercoris sp. nov.}

5345

Candidatus Intestinimonas pullistercoris (pul.li.ster'co.ris. L. masc. n. pullus a young chicken; L.

5346

5347

5348

5349

5350

5351

5352 neut. n. stercus dung; N.L. gen. n. pullistercoris of young chicken faeces)

5353

A bacterial species identified by metagenomic analyses. This species includes all bacteria with genomes that show $\geq 95 \%$ average nucleotide identity (ANI) to the type genome, which has been assigned the MAG ID CHK186-1790 and which is available via NCBI BioSample

SAMN15816581. The GC content of the type genome is $64.76 \%$ and the genome length is 2.4 Mbp. 
5355 Description of Candidatus Intestinimonas stercoravium sp. nov.

5356 Candidatus Intestinimonas stercoravium (ster.cor.a'vi.um. L. neut. n. stercus dung; L. fem. n.

5357 avis bird; N.L. gen. n. stercoravium of bird faeces)

5358

5359

A bacterial species identified by metagenomic analyses. This species includes all bacteria with

5360

5361 genomes that show $\geq 95 \%$ average nucleotide identity (ANI) to the type genome, which has been assigned the MAG ID ChiBcolR2-622 and which is available via NCBI BioSample

5362

5363

SAMN15816599. The GC content of the type genome is $65.33 \%$ and the genome length is 2.2 Mbp.

5364

5365

5366

Description of Candidatus Intestinimonas stercorigallinarum sp. nov.

5367 Candidatus Intestinimonas stercorigallinarum (ster.co.ri.gal.li.na'rum. L. neut. n. stercus dung; L. 5368 fem. n. gallina hen; N.L. gen. n. stercorigallinarum of hen faeces)

5369

5370

A bacterial species identified by metagenomic analyses. This species includes all bacteria with

5371

5372

5373

5374

5375 genomes that show $\geq 95 \%$ average nucleotide identity (ANI) to the type genome, which has been assigned the MAG ID ChiSxjej2B14-1745 and which is available via NCBI BioSample SAMN15816719. The GC content of the type genome is $65.69 \%$ and the genome length is 2.1 Mbp.

5376

5377

5378

Description of Candidatus Janibacter merdipullorum sp. nov.

Candidatus Janibacter merdipullorum (mer.di.pul.lo'rum. L. fem. n. merda faeces; L. masc. n.

5379

pullus a young chicken; N.L. gen. n. merdipullorum of the faeces of young chickens)

5380

5381

5382

A bacterial species identified by metagenomic analyses. This species includes all bacteria with genomes that show $\geq 95 \%$ average nucleotide identity (ANI) to the type genome, which has been

5383 assigned the MAG ID ChiHjej13B12-21492 and which is available via NCBI BioSample

5384

5385 SAMN15816685. The GC content of the type genome is $71.49 \%$ and the genome length is 2.8

5386

5387

5388 Mbp.

5389

Description of Candidatus Jeotgalibaca merdavium sp. nov.

5390

Candidatus Jeotgalibaca merdavium (merd.a'vi.um. L. fem. n. merda faeces; L. fem. n. avis bird;

5391

5392

N.L. gen. n. merdavium of bird faeces)

5393

A bacterial species identified by metagenomic analyses. This species includes all bacteria with genomes that show $\geq 95 \%$ average nucleotide identity (ANI) to the type genome, which has been

5394

5395 assigned the MAG ID CHK171-505 and which is available via NCBI BioSample

5396

5397

SAMN15816828. This is a new name for the alphanumeric GTDB species sp001975685. The

GC content of the type genome is $38.39 \%$ and the genome length is $2.0 \mathrm{Mbp}$.

5398

5399

Description of Candidatus Jeotgalibaca pullicola sp. nov.

Peer) reviewing PDF | (2020:12:55980:1:1:NEW 13 Jan 2021) 
5400 Candidatus Jeotgalibaca pullicola (pul.li'co.la. L. masc. n. pullus a young chicken; L. suff. -cola 5401 inhabitant of; N.L. n. pullicola an inhabitant of young chickens)

5402

5403

5404

5405

5406

5407

5408

5409

5410

5411

5412

5413

5414

5415

5416

5417

5418

5419

5420

5421

5422

5423

5424

5425

5426

5427

5428

5429

5430

5431

5432

5433

5434

5435

5436

5437

5438

5439

5440

5441

5442

5443

5444

5445

A bacterial species identified by metagenomic analyses. This species includes all bacteria with genomes that show $\geq 95 \%$ average nucleotide identity (ANI) to the type genome, which has been assigned the MAG ID CHK172-9797 and which is available via NCBI BioSample

SAMN15816826. This is a new name for the alphanumeric GTDB species sp003955755. The GC content of the type genome is $36.93 \%$ and the genome length is $2.6 \mathrm{Mbp}$.

\section{Description of Candidatus Jeotgalicoccus stercoravium sp. nov.}

Candidatus Jeotgalicoccus stercoravium (ster.cor.a'vi.um. L. neut. n. stercus dung; L. fem. $n$. avis bird; N.L. gen. n. stercoravium of bird faeces)

A bacterial species identified by metagenomic analyses. This species includes all bacteria with genomes that show $\geq 95 \%$ average nucleotide identity (ANI) to the type genome, which has been assigned the MAG ID CHK148-7025 and which is available via NCBI BioSample SAMN15816765. The GC content of the type genome is $36.17 \%$ and the genome length is 1.7 Mbp.

\section{Description of Candidatus Kurthia intestinigallinarum sp. nov.}

Candidatus Kurthia intestinigallinarum (in.tes.ti.ni.gal.li.na'rum. L. neut. n. intestinum gut; L. fem. n. gallina hen; N.L. gen. n. intestinigallinarum of the gut of the hens)

A bacterial species identified by metagenomic analyses. This species includes all bacteria with genomes that show $\geq 95 \%$ average nucleotide identity (ANI) to the type genome, which has been assigned the MAG ID CHK171-3164 and which is available via NCBI BioSample SAMN15816863. This is a new name for the alphanumeric GTDB species sp002418445. The GC content of the type genome is $39.62 \%$ and the genome length is $2.9 \mathrm{Mbp}$.

\section{Description of Candidatus Lachnoclostridium avicola sp. nov.}

Candidatus Lachnoclostridium avicola (a.vi'co.la. L. fem. n. avis bird; L. suff. -cola inhabitant of; N.L. n. avicola inhabitant of birds)

A bacterial species identified by metagenomic analyses. This species includes all bacteria with genomes that show $\geq 95 \%$ average nucleotide identity (ANI) to the type genome, which has been assigned the MAG ID CHK190-19777 and which is available via NCBI BioSample SAMN15816889. Although GTDB has assigned this species to the genus it calls Lachnoclostridium_A, this genus designation cannot be incorporated into a well-formed binomial, so in naming this species, we have used the current validly published name for the genus. The GC content of the type genome is $54.75 \%$ and the genome length is $2.9 \mathrm{Mbp}$.

\section{Description of Candidatus Lachnoclostridium pullistercoris sp. nov.}


5446 Candidatus Lachnoclostridium pullistercoris (pul.li.ster'co.ris. L. masc. n. pullus a young

5447 chicken; L. neut. n. stercus dung; N.L. gen. n. pullistercoris of young chicken faeces)

5448

5449

A bacterial species identified by metagenomic analyses. This species includes all bacteria with

5450

5451

5452

5453

5454

5455

5456

5457

5458

5459

5460

5461

5462

5463

5464

5465

5466

5467

5468

5469

5470

5471

5472

5473

5474

5475

5476

5477

5478

5479

5480

5481

5482

5483

5484

5485 genomes that show $\geq 95 \%$ average nucleotide identity (ANI) to the type genome, which has been assigned the MAG ID CHK183-5548 and which is available via NCBI BioSample SAMN15816884. Although GTDB has assigned this species to the genus it calls Lachnoclostridium_A, this genus designation cannot be incorporated into a well-formed binomial, so in naming this species, we have used the current validly published name for the genus. The GC content of the type genome is $54.35 \%$ and the genome length is $2.8 \mathrm{Mbp}$.

\section{Description of Candidatus Lachnoclostridium stercoravium sp. nov.}

Candidatus Lachnoclostridium stercoravium (ster.cor.a'vi.um. L. neut. n. stercus dung; L. fem. n. avis bird; N.L. gen. n. stercoravium of bird faeces)

A bacterial species identified by metagenomic analyses. This species includes all bacteria with genomes that show $\geq 95 \%$ average nucleotide identity (ANI) to the type genome, which has been assigned the MAG ID CHK178-16964 and which is available via NCBI BioSample SAMN15816887. Although GTDB has assigned this species to the genus it calls Lachnoclostridium_A, this genus designation cannot be incorporated into a well-formed binomial, so in naming this species, we have used the current validly published name for the genus. The GC content of the type genome is $49.55 \%$ and the genome length is $3.0 \mathrm{Mbp}$.

\section{Description of Candidatus Lachnoclostridium stercorigallinarum sp. nov.}

Candidatus Lachnoclostridium stercorigallinarum (ster.co.ri.gal.li.na'rum. L. neut. n. stercus dung; L. fem. n. gallina hen; N.L. gen. n. stercorigallinarum of hen faeces)

A bacterial species identified by metagenomic analyses. This species includes all bacteria with genomes that show $\geq 95 \%$ average nucleotide identity (ANI) to the type genome, which has been assigned the MAG ID ChiBcec1-1093 and which is available via NCBI BioSample SAMN15816897. Although GTDB has assigned this species to the genus it calls Lachnoclostridium_A, this genus designation cannot be incorporated into a well-formed binomial, so in naming this species, we have used the current validly published name for the genus. The GC content of the type genome is $54.29 \%$ and the genome length is $2.4 \mathrm{Mbp}$.

\section{Description of Candidatus Lachnoclostridium stercoripullorum sp. nov.} Candidatus Lachnoclostridium stercoripullorum (ster.co.ri.pul.lo'rum. L. neut. n. stercus dung; L. masc. n. pullus a young chicken; N.L. gen. n. stercoripullorum of the faceces of young chickens)

A bacterial species identified by metagenomic analyses. This species includes all bacteria with 5491 genomes that show $\geq 95 \%$ average nucleotide identity (ANI) to the type genome, which has been assigned the MAG ID ChiGjej4B4-12881 and which is available via NCBI BioSample 
5492 SAMN15816908. Although GTDB has assigned this species to the genus it calls

5493 Lachnoclostridium_A, this genus designation cannot be incorporated into a well-formed

5494 binomial, so in naming this species, we have used the current validly published name for the

5495 genus. The GC content of the type genome is $59.38 \%$ and the genome length is $2.3 \mathrm{Mbp}$.

5496

5497

5498

5499

Description of Candidatus Lactobacillus pullistercoris sp. nov.

5500

5501

5502

Candidatus Lactobacillus pullistercoris (pul.li.ster'co.ris. L. masc. n. pullus a young chicken; L. neut. n. stercus dung; N.L. gen. n. pullistercoris of young chicken faeces)

5503

A bacterial species identified by metagenomic analyses. This species includes all bacteria with

5504 genomes that show $\geq 95 \%$ average nucleotide identity (ANI) to the type genome, which has been assigned the MAG ID F6-686 and which is available via NCBI BioSample SAMN15816686.

5505 The GC content of the type genome is $34.54 \%$ and the genome length is $1.7 \mathrm{Mbp}$.

5506

5507

5508

Description of Candidatus Lawsonibacter pullicola sp. nov.

5509

Candidatus Lawsonibacter pullicola (pul.li'co.la. L. masc. n. pullus a young chicken; L. suff. -

5510 cola inhabitant of; N.L. n. pullicola an inhabitant of young chickens)

5511

5512

A bacterial species identified by metagenomic analyses. This species includes all bacteria with

5513

5514

5515 genomes that show $\geq 95 \%$ average nucleotide identity (ANI) to the type genome, which has been assigned the MAG ID CHK178-3907 and which is available via NCBI BioSample SAMN15816869. This is a new name for the alphanumeric GTDB species sp002160305. The

5516

5517 $\mathrm{GC}$ content of the type genome is $62.98 \%$ and the genome length is $2.3 \mathrm{Mbp}$.

5518

5519

Description of Candidatus Levilactobacillus faecigallinarum sp. nov.

5520

Candidatus Levilactobacillus faecigallinarum (fae.ci.gal.li.na'rum. L. fem. n. faex, faecis

5521

5522

excrement; L. fem. n. gallina hen; N.L. gen. n. faecigallinarum of hen faeces)

5523

5524

5525

A bacterial species identified by metagenomic analyses. This species includes all bacteria with genomes that show $\geq 95 \%$ average nucleotide identity (ANI) to the type genome, which has been assigned the MAG ID CHK173-259 and which is available via NCBI BioSample

5526

5527

5528

5529

5530 SAMN15816755. The GC content of the type genome is $52.18 \%$ and the genome length is 1.8 Mbp.

5531

\section{Description of Candidatus Ligilactobacillus avistercoris sp. nov.}

Candidatus Ligilactobacillus avistercoris (a.vi.ster'co.ris. L. fem. n. avis bird; L. neut. n. stercus

5532

5533

5534 dung; N.L. gen. n. avistercoris of bird faeces)

5535

A bacterial species identified by metagenomic analyses. This species includes all bacteria with genomes that show $\geq 95 \%$ average nucleotide identity (ANI) to the type genome, which has been 5536 assigned the MAG ID ChiBile7-59 and which is available via NCBI BioSample 
5537 SAMN15816642. The GC content of the type genome is $51.08 \%$ and the genome length is 1.2 $5538 \mathrm{Mbp}$.

5539

5540

5541

5542

5543

5544

5545

5546

5547

5548

5549

5550

5551

5552

5553

5554

5555

5556

5557

5558

5559

5560

5561

5562

Description of Candidatus Ligilactobacillus excrementavium sp. nov.

Candidatus Ligilactobacillus excrementavium (ex.cre.ment.a'vi.um. L. neut. n. excrementum excrement; L. fem. n. avis bird; N.L. gen. n. excrementavium of bird excrement)

A bacterial species identified by metagenomic analyses. This species includes all bacteria with genomes that show $\geq 95 \%$ average nucleotide identity (ANI) to the type genome, which has been assigned the MAG ID 2259 and which is available via NCBI BioSample SAMN15816683. The $\mathrm{GC}$ content of the type genome is $38.00 \%$ and the genome length is $1.9 \mathrm{Mbp}$.

\section{Description of Candidatus Ligilactobacillus excrementigallinarum sp. nov.}

Candidatus Ligilactobacillus excrementigallinarum (ex.cre.men.ti.gal.li.na'rum. L. neut. n. excrementum excrement; L. fem. n. gallina hen; N.L. gen. n. excrementigallinarum of hen excrement)

A bacterial species identified by metagenomic analyses. This species includes all bacteria with genomes that show $\geq 95 \%$ average nucleotide identity (ANI) to the type genome, which has been assigned the MAG ID 6627 and which is available via NCBI BioSample SAMN15816741. The $\mathrm{GC}$ content of the type genome is $34.16 \%$ and the genome length is $1.2 \mathrm{Mbp}$.

5563

Description of Candidatus Ligilactobacillus excrementipullorum sp. nov. Candidatus Ligilactobacillus excrementipullorum (ex.cre.men.ti.pul.lo'rum. L. neut. n.

5564 excrementum excrement; L. masc. n. pullus a young chicken; N.L. gen. n. excrementipullorum of

5565

5566

5567

5568

5569

5570

5571 young chicken excrement)

5572

5573

5574

5575

A bacterial species identified by metagenomic analyses. This species includes all bacteria with genomes that show $\geq 95 \%$ average nucleotide identity (ANI) to the type genome, which has been assigned the MAG ID CHK171-2193 and which is available via NCBI BioSample SAMN15816749. The GC content of the type genome is $42.06 \%$ and the genome length is 2.0 Mbp.

5576

5577

5578

5579

5580

5581

5582

Description of Candidatus Ligilactobacillus faecavium sp. nov.

Candidatus Ligilactobacillus faecavium (faec.a'vi.um. L. fem. n. faex, faecis excrement; L. fem. n. avis bird; N.L. gen. n. faecavium of bird faeces)

A bacterial species identified by metagenomic analyses. This species includes all bacteria with genomes that show $\geq 95 \%$ average nucleotide identity (ANI) to the type genome, which has been assigned the MAG ID 3439 and which is available via NCBI BioSample SAMN15816798. The $\mathrm{GC}$ content of the type genome is $40.05 \%$ and the genome length is $1.3 \mathrm{Mbp}$. 
5583

5584

5585

5586

5587

5588

5589

5590

5591

5592

5593

5594

5595

5596

5597

5598

5599

5600

5601

5602

5603

5604

5605

5606

5607

5608

5609

5610

5611

5612

5613

5614

5615

5616

5617

5618

5619

5620

5621

5622

5623

5624

5625

5626

5627

5628

Description of Candidatus Limadaptatus gen. nov.

Candidatus Limadaptatus (Lim.adaptatus. L. masc. n. limus dung; L. past part. masc. adaptatus adapted to; N.L. masc. n. Limadaptatus a microbe associated with faeces)

A bacterial genus identified by metagenomic analyses. The genus includes all bacteria with genomes that show $\geq 60 \%$ average amino acid identity (AAI) to the type genome from the type species Limadaptatus stercoripullorum. This is a name for the alphanumeric GTDB genus UMGS1688. This genus has been assigned by GTDB-Tk v1.3.0 working on GTDB Release 05RS95 (Chaumeil et al., 2019; Parks et al., 2020) to the order Christensenellales and to the family CAG-917.

\section{Description of Candidatus Limadaptatus stercoravium sp. nov.}

Candidatus Limadaptatus stercoravium (ster.cor.a'vi.um. L. neut. n. stercus dung; L. fem. n. avis bird; N.L. gen. n. stercoravium of bird faeces)

A bacterial species identified by metagenomic analyses. This species includes all bacteria with genomes that show $\geq 95 \%$ average nucleotide identity (ANI) to the type genome, which has been assigned the MAG ID CHK154-227 and which is available via NCBI BioSample SAMN15817097. The GC content of the type genome is $58.03 \%$ and the genome length is 1.4 Mbp.

\section{Description of Candidatus Limadaptatus stercorigallinarum sp. nov.}

Candidatus Limadaptatus stercorigallinarum (ster.co.ri.gal.li.na'rum. L. neut. n. stercus dung; L. fem. n. gallina hen; N.L. gen. n. stercorigallinarum of hen faeces)

A bacterial species identified by metagenomic analyses. This species includes all bacteria with genomes that show $\geq 95 \%$ average nucleotide identity (ANI) to the type genome, which has been assigned the MAG ID 1063 and which is available via NCBI BioSample SAMN15817231. This is a new name for the alphanumeric GTDB species sp900544575. The GC content of the type genome is $56.41 \%$ and the genome length is $1.5 \mathrm{Mbp}$.

\section{Description of Candidatus Limadaptatus stercoripullorum sp. nov.}

Candidatus Limadaptatus stercoripullorum (ster.co.ri.pul.lo'rum. L. neut. n. stercus dung; L. masc. n. pullus a young chicken; N.L. gen. n. stercoripullorum of the faceces of young chickens)

A bacterial species identified by metagenomic analyses. This species includes all bacteria with genomes that show $\geq 95 \%$ average nucleotide identity (ANI) to the type genome, which has been assigned the MAG ID 10406 and which is available via NCBI BioSample SAMN15817154. The $\mathrm{GC}$ content of the type genome is $61.19 \%$ and the genome length is $1.4 \mathrm{Mbp}$.

\section{Description of Candidatus Limenecus gen. nov.}


5629 Candidatus Limenecus (Lim.en.e'cus. L. masc. n. limus dung; Gr. masc. enoikos inhabitant; N.L.

5630

5631

5632

5633

5634

5635

5636

5637

5638

5639

5640

5641

5642

5643

5644

5645

5646

5647

5648

5649

5650

5651

5652

5653

5654

5655

5656

5657

5658

5659

5660

5661

5662

5663

5664

5665

5666

5667

5668

5669

5670

5671

5672

5673

5674

masc. n. Limenecus a microbe associated with faeces)

A bacterial genus identified by metagenomic analyses. The genus includes all bacteria with genomes that show $\geq 60 \%$ average amino acid identity (AAI) to the type genome from the type species Limenecus avicola. This is a name for the alphanumeric GTDB genus CAG-306. This genus has been assigned by GTDB-Tk v1.3.0 working on GTDB Release 05-RS95 (Chaumeil et al., 2019; Parks et al., 2020) to the order Gastranaerophilales and to the family

Gastranaerophilaceae.

\section{Description of Candidatus Limenecus avicola sp. nov.}

Candidatus Limenecus avicola (a.vi'co.la. L. fem. n. avis bird; L. suff. -cola inhabitant of; N.L. n. avicola inhabitant of birds)

A bacterial species identified by metagenomic analyses. This species includes all bacteria with genomes that show $\geq 95 \%$ average nucleotide identity (ANI) to the type genome, which has been assigned the MAG ID CHK154-7741 and which is available via NCBI BioSample SAMN15817206. This is a new name for the alphanumeric GTDB species sp000980375. The GC content of the type genome is $36.63 \%$ and the genome length is $2.1 \mathrm{Mbp}$.

\section{Description of Candidatus Limicola gen. nov.}

Candidatus Limicola (Lim.i.co'la. L. masc. n. limus dung; L. suff. -cola inhabitant of; N.L. fem. n. Limicola a microbe associated with faeces)

A bacterial genus identified by metagenomic analyses. The genus includes all bacteria with genomes that show $\geq 60 \%$ average amino acid identity (AAI) to the type genome from the type species Limicola stercorigallinarum. This is a name for the alphanumeric GTDB genus An2-A. This genus has been assigned by GTDB-Tk v1.3.0 working on GTDB Release 05-RS95 (Chaumeil et al., 2019; Parks et al., 2020) to the order Coriobacteriales and to the family Coriobacteriaceae.

\section{Description of Candidatus Limicola stercorigallinarum sp. nov.}

Candidatus Limicola stercorigallinarum (ster.co.ri.gal.li.na'rum. L. neut. n. stercus dung; L. fem. n. gallina hen; N.L. gen. n. stercorigallinarum of hen faeces)

A bacterial species identified by metagenomic analyses. This species includes all bacteria with genomes that show $\geq 95 \%$ average nucleotide identity (ANI) to the type genome, which has been assigned the MAG ID ChiGjej1B1-16188 and which is available via NCBI BioSample SAMN15817082. The GC content of the type genome is $60.48 \%$ and the genome length is 2.0 Mbp.

\section{Description of Candidatus Limihabitans gen. nov.}


5675 Candidatus Limihabitans (Lim.i.hab.it'ans. L. masc. n. limus dung; L. pres. part. habitans an

5676

5677

5678

5679

5680

5681

5682

5683

5684

5685

5686

5687

5688

5689

5690

5691

5692

5693

5694

5695

5696

5697

5698

5699

5700

5701

5702

5703

5704

5705

5706

5707

5708

5709

5710

5711

5712

5713

5714

5715

5716

5717

5718

5719

inhabitant; N.L. fem. n. Limihabitans a microbe associated with faeces)

A bacterial genus identified by metagenomic analyses. The genus includes all bacteria with genomes that show $\geq 60 \%$ average amino acid identity (AAI) to the type genome from the type species Limihabitans stercoravium. This is a name for the alphanumeric GTDB genus UMGS1707. This genus has been assigned by GTDB-Tk v1.3.0 working on GTDB Release 05RS95 (Chaumeil et al., 2019; Parks et al., 2020) to the order $4 C 28 d-15$ and to the family $C A G$ 314.

\section{Description of Candidatus Limihabitans stercoravium sp. nov.}

Candidatus Limihabitans stercoravium (ster.cor.a'vi.um. L. neut. n. stercus dung; L. fem. n. avis bird; N.L. gen. n. stercoravium of bird faeces)

A bacterial species identified by metagenomic analyses. This species includes all bacteria with genomes that show $\geq 95 \%$ average nucleotide identity (ANI) to the type genome, which has been assigned the MAG ID 3394 and which is available via NCBI BioSample SAMN15817227. This is a new name for the alphanumeric GTDB species sp900547645. The GC content of the type genome is $43.23 \%$ and the genome length is $1.6 \mathrm{Mbp}$.

\section{Description of Candidatus Limimorpha gen. nov.}

Candidatus Limimorpha (Lim.i.mor'pha. L. masc. n. limus dung; Gr. fem. n. morphe a form, shape; N.L. fem. n. Limimorpha a microbe associated with faeces)

A bacterial genus identified by metagenomic analyses. The genus includes all bacteria with genomes that show $\geq 60 \%$ average amino acid identity (AAI) to the type genome from the type species Limimorpha avicola. This is a name for the alphanumeric GTDB genus F082. This genus has been assigned by GTDB-Tk v1.3.0 working on GTDB Release 05-RS95 (Chaumeil et al., 2019; Parks et al., 2020) to the order Bacteroidales and to the family F082.

\section{Description of Candidatus Limimorpha avicola sp. nov.}

Candidatus Limimorpha avicola (a.vi'co.la. L. fem. n. avis bird; L. suff. -cola inhabitant of; N.L. n. avicola inhabitant of birds)

A bacterial species identified by metagenomic analyses. This species includes all bacteria with genomes that show $\geq 95 \%$ average nucleotide identity (ANI) to the type genome, which has been assigned the MAG ID Gambia15-481 and which is available via NCBI BioSample SAMN15817194. This is a new name for the alphanumeric GTDB species sp002633315. The $\mathrm{GC}$ content of the type genome is $38.09 \%$ and the genome length is $2.5 \mathrm{Mbp}$.

\section{Description of Candidatus Limiplasma gen. nov.}


5720 Candidatus Limiplasma (Lim.i.plas'ma. L. masc. n. limus dung; Gr. neut. n. plasma a form; N.L.

5721

5722

5723

5724

5725

5726

5727

5728

5729

5730

5731

5732

5733

5734

5735

5736

5737

5738

5739

5740

5741

5742

5743

5744

5745

5746

5747

5748

5749

5750

5751

5752

5753

5754

5755

5756

5757

5758

5759

5760

5761

5762

5763

5764

5765

neut. n. Limiplasma a microbe associated with faeces)

A bacterial genus identified by metagenomic analyses. The genus includes all bacteria with genomes that show $\geq 60 \%$ average amino acid identity (AAI) to the type genome from the type species Limiplasma pullicola. This is a name for the alphanumeric GTDB genus Firm-11. This genus has been assigned by GTDB-Tk v1.3.0 working on GTDB Release 05-RS95 (Chaumeil et al., 2019; Parks et al., 2020) to the order Christensenellales and to the family CAG-74.

\section{Description of Candidatus Limiplasma merdipullorum sp. nov.}

Candidatus Limiplasma merdipullorum (mer.di.pul.lo'rum. L. fem. n. merda faeces; L. masc. n. pullus a young chicken; N.L. gen. n. merdipullorum of the faeces of young chickens)

A bacterial species identified by metagenomic analyses. This species includes all bacteria with genomes that show $\geq 95 \%$ average nucleotide identity (ANI) to the type genome, which has been assigned the MAG ID ChiBcec16-3123 and which is available via NCBI BioSample SAMN15817217. This is a new name for the alphanumeric GTDB species sp900540045. The $\mathrm{GC}$ content of the type genome is $61.78 \%$ and the genome length is $2.6 \mathrm{Mbp}$.

\section{Description of Candidatus Limiplasma pullicola sp. nov.}

Candidatus Limiplasma pullicola (pul.li'co.la. L. masc. n. pullus a young chicken; L. suff. -cola inhabitant of; N.L. n. pullicola an inhabitant of young chickens)

A bacterial species identified by metagenomic analyses. This species includes all bacteria with genomes that show $\geq 95 \%$ average nucleotide identity (ANI) to the type genome, which has been assigned the MAG ID 2223 and which is available via NCBI BioSample SAMN15817092. The GC content of the type genome is $62.99 \%$ and the genome length is $2.7 \mathrm{Mbp}$.

\section{Description of Candidatus Limiplasma pullistercoris sp. nov.}

Candidatus Limiplasma pullistercoris (pul.li.ster'co.ris. L. masc. n. pullus a young chicken; L. neut. n. stercus dung; N.L. gen. n. pullistercoris of young chicken faeces)

A bacterial species identified by metagenomic analyses. This species includes all bacteria with genomes that show $\geq 95 \%$ average nucleotide identity (ANI) to the type genome, which has been assigned the MAG ID ChiBcec15-2748 and which is available via NCBI BioSample SAMN15817228. This is a new name for the alphanumeric GTDB species sp900553905. The GC content of the type genome is $62.75 \%$ and the genome length is $2.8 \mathrm{Mbp}$.

\section{Description of Candidatus Limiplasma stercoravium sp. nov.}

Candidatus Limiplasma stercoravium (ster.cor.a'vi.um. L. neut. n. stercus dung; L. fem. n. avis bird; N.L. gen. n. stercoravium of bird faeces) 
5766 A bacterial species identified by metagenomic analyses. This species includes all bacteria with 5767 genomes that show $\geq 95 \%$ average nucleotide identity (ANI) to the type genome, which has been 5768 assigned the MAG ID CHK169-20388 and which is available via NCBI BioSample

5769 SAMN15817129. The GC content of the type genome is $62.11 \%$ and the genome length is 2.4

5770 Mbp.

5771

5772

5773

5774

5775

5776

5777

5778

5779

5780

5781

5782

5783

5784

5785

5786

5787

5788

5789

5790

5791

5792

5793

5794

5795

5796

Description of Candidatus Limisoma gen. nov.

Candidatus Limisoma (Lim.i.so'ma. L. masc. n. limus dung; Gr. neut. n. soma a body; N.L. neut. n. Limisoma a microbe associated with faeces)

A bacterial genus identified by metagenomic analyses. The genus includes all bacteria with genomes that show $\geq 60 \%$ average amino acid identity (AAI) to the type genome from the type species Limisoma faecipullorum. This is a name for the alphanumeric GTDB genus CAG-279. This genus has been assigned by GTDB-Tk v1.3.0 working on GTDB Release 05-RS95 (Chaumeil et al., 2019; Parks et al., 2020) to the order Bacteroidales and to the family Muribaculaceae.

\section{Description of Candidatus Limisoma faecipullorum sp. nov.}

Candidatus Limisoma faecipullorum (fae.ci.pul.lo'rum. L. fem. n. faex, faecis excrement; L. masc. n. pullus a young chicken; N.L. gen. n. faecipullorum of young chicken faeces)

A bacterial species identified by metagenomic analyses. This species includes all bacteria with genomes that show $\geq 95 \%$ average nucleotide identity (ANI) to the type genome, which has been assigned the MAG ID 6919 and which is available via NCBI BioSample SAMN15817069. The GC content of the type genome is $45.95 \%$ and the genome length is $2.2 \mathrm{Mbp}$.

\section{Description of Candidatus Limisoma gallistercoris sp. nov.}

Candidatus Limisoma gallistercoris (gal.li.ster'co.ris. L. masc. $\mathrm{n}$ gallus chicken; L. neut. n.

5797

5798

5799 stercus dung; N.L. gen. n. gallistercoris of chicken faeces)

5800

5801

5802

5803

5804

5805

5806

5807

5808

5809

5810 A bacterial species identified by metagenomic analyses. This species includes all bacteria with

5811

A bacterial species identified by metagenomic analyses. This species includes all bacteria with genomes that show $\geq 95 \%$ average nucleotide identity (ANI) to the type genome, which has been assigned the MAG ID CHK136-1475 and which is available via NCBI BioSample SAMN15817174. This is a new name for the alphanumeric GTDB species sp900550025. The GC content of the type genome is $48.31 \%$ and the genome length is $2.2 \mathrm{Mbp}$.

\section{Description of Candidatus Limisoma intestinavium sp. nov.}

Candidatus Limisoma intestinavium (in.tes.tin.a'vi.um. L. neut. n. intestinum gut; L. fem. n. avis bird; N.L. gen. n. intestinavium of the gut of birds) genomes that show $\geq 95 \%$ average nucleotide identity (ANI) to the type genome, which has been 
5812 assigned the MAG ID 17073 and which is available via NCBI BioSample SAMN15817199. This 5813 is a new name for the alphanumeric GTDB species sp900541555. The GC content of the type 5814 genome is $48.25 \%$ and the genome length is $1.8 \mathrm{Mbp}$.

5815

5816

5817

\section{Description of Candidatus Limivicinus gen. nov.}

5818 Candidatus Limivicinus (Lim.i.vic.in'us. L. masc. n. limus dung; L. masc. n. vicinus a neighbour; 5819 5820

5821

5822

5823

5824

5825

5826

5827

5828

5829

5830

5831

5832

5833

5834

5835

5836

5837

5838

5839

5840

5841

5842

5843

5844

5845

5846

5847

5848

5849

5850 N.L. masc. n. Limivicinus a microbe associated with faeces)

A bacterial genus identified by metagenomic analyses. The genus includes all bacteria with genomes that show $\geq 60 \%$ average amino acid identity (AAI) to the type genome from the type species Limivicinus faecipullorum. This is a name for the alphanumeric GTDB genus UBA1777. This genus has been assigned by GTDB-Tk v1.3.0 working on GTDB Release 05-RS95 (Chaumeil et al., 2019; Parks et al., 2020) to the order Oscillospirales and to the family Oscillospiraceae.

\section{Description of Candidatus Limivicinus faecipullorum sp. nov.}

Candidatus Limivicinus faecipullorum (fae.ci.pul.lo'rum. L. fem. n. faex, faecis excrement; L. masc. n. pullus a young chicken; N.L. gen. n. faecipullorum of young chicken faeces)

A bacterial species identified by metagenomic analyses. This species includes all bacteria with genomes that show $\geq 95 \%$ average nucleotide identity (ANI) to the type genome, which has been assigned the MAG ID ChiHcec3-8852 and which is available via NCBI BioSample SAMN15817081. The GC content of the type genome is $58.52 \%$ and the genome length is 1.9 Mbp.

\section{Description of Candidatus Limivivens gen. nov.}

Candidatus Limivivens (Lim.i.viv'ens. L. masc. n. limus dung; N.L. pres. part. vivens living; N.L. fem. n. Limivivens a microbe associated with faeces)

A bacterial genus identified by metagenomic analyses. The genus includes all bacteria with genomes that show $\geq 60 \%$ average amino acid identity (AAI) to the type genome from the type species Limivivens intestinipullorum. This is a name for the alphanumeric GTDB genus GCA900066135. This genus has been assigned by GTDB-Tk v1.3.0 working on GTDB Release 05RS95 (Chaumeil et al., 2019; Parks et al., 2020) to the order Lachnospirales and to the family Lachnospiraceae.

5851

5852

5853

5854

5855

5856

5857

\section{Description of Candidatus Limivivens intestinipullorum sp. nov.}

Candidatus Limivivens intestinipullorum (in.tes.ti.ni.pul.lo'rum. L. neut. n. intestinum gut; L. masc. n. pullus a young chicken; N.L. gen. n. intestinipullorum of the gut of young chickens)

A bacterial species identified by metagenomic analyses. This species includes all bacteria with genomes that show $\geq 95 \%$ average nucleotide identity (ANI) to the type genome, which has been 
5858 assigned the MAG ID CHK190-19873 and which is available via NCBI BioSample

5859 SAMN15817025. The GC content of the type genome is $52.58 \%$ and the genome length is 3.4

5860 Mbp.

5861

5862

5863

5864

5865

5866

5867

5868

5869

5870

5871

5872

5873

5874

5875

5876

5877

5878

5879

5880

5881

5882

5883

5884

5885

5886

5887

5888

5889

5890

5891

5892

5893

5894

5895

5896

5897

5898

5899

5900

5901

5902

\section{Description of Candidatus Limivivens merdigallinarum sp. nov.}

Candidatus Limivivens merdigallinarum (mer.di.gal.li.na'rum. L. fem. n. merda faeces; L. fem. n. gallina hen; N.L. gen. n. merdigallinarum of hen faeces)

A bacterial species identified by metagenomic analyses. This species includes all bacteria with genomes that show $\geq 95 \%$ average nucleotide identity (ANI) to the type genome, which has been assigned the MAG ID ChiSjej3B21-11622 and which is available via NCBI BioSample SAMN15817007. The GC content of the type genome is $50.09 \%$ and the genome length is 3.3 Mbp.

\section{Description of Candidatus Limosilactobacillus excrementigallinarum sp. nov.}

Candidatus Limosilactobacillus excrementigallinarum (ex.cre.men.ti.gal.li.na'rum. L. neut. n. excrementum excrement; L. fem. n. gallina hen; N.L. gen. n. excrementigallinarum of hen excrement)

A bacterial species identified by metagenomic analyses. This species includes all bacteria with genomes that show $\geq 95 \%$ average nucleotide identity (ANI) to the type genome, which has been assigned the MAG ID 2685 and which is available via NCBI BioSample SAMN15816672. The $\mathrm{GC}$ content of the type genome is $41.67 \%$ and the genome length is $1.4 \mathrm{Mbp}$.

\section{Description of Candidatus Limosilactobacillus faecipullorum sp. nov.}

Candidatus Limosilactobacillus faecipullorum (fae.ci.pul.lo'rum. L. fem. n. faex, faecis excrement; L. masc. n. pullus a young chicken; N.L. gen. n. faecipullorum of young chicken faeces)

A bacterial species identified by metagenomic analyses. This species includes all bacteria with genomes that show $\geq 95 \%$ average nucleotide identity (ANI) to the type genome, which has been assigned the MAG ID 7774 and which is available via NCBI BioSample SAMN15816663. The GC content of the type genome is $43.03 \%$ and the genome length is $1.5 \mathrm{Mbp}$.

\section{Description of Candidatus Limosilactobacillus gallistercoris sp. nov.} Candidatus Limosilactobacillus gallistercoris (gal.li.ster'co.ris. L. masc. $\mathrm{n}$ gallus chicken; L. neut. n. stercus dung; N.L. gen. n. gallistercoris of chicken faeces)

A bacterial species identified by metagenomic analyses. This species includes all bacteria with genomes that show $\geq 95 \%$ average nucleotide identity (ANI) to the type genome, which has been assigned the MAG ID CHK158-2993 and which is available via NCBI BioSample 
5903 SAMN15816598. The GC content of the type genome is $52.74 \%$ and the genome length is 1.2 $5904 \mathrm{Mbp}$.

5905

5906

5907

Description of Candidatus Limosilactobacillus intestinavium sp. nov.

5908

Candidatus Limosilactobacillus intestinavium (in.tes.tin.a'vi.um. L. neut. n. intestinum gut; L.

5909 fem. n. avis bird; N.L. gen. n. intestinavium of the gut of birds)

5910

5911

A bacterial species identified by metagenomic analyses. This species includes all bacteria with

5912 genomes that show $\geq 95 \%$ average nucleotide identity (ANI) to the type genome, which has been

5913 assigned the MAG ID 2331 and which is available via NCBI BioSample SAMN15816838. This

5914

5915

5916

5917

5918

5919 is a new name for the alphanumeric GTDB species sp900557215. The GC content of the type genome is $38.77 \%$ and the genome length is $1.5 \mathrm{Mbp}$.

\section{Description of Candidatus Limosilactobacillus intestinigallinarum sp. nov.} Candidatus Limosilactobacillus intestinigallinarum (in.tes.ti.ni.gal.li.na'rum. L. neut. n. intestinum gut; L. fem. n. gallina hen; N.L. gen. n. intestinigallinarum of the gut of the hens)

5921

5922

A bacterial species identified by metagenomic analyses. This species includes all bacteria with genomes that show $\geq 95 \%$ average nucleotide identity (ANI) to the type genome, which has been assigned the MAG ID CHK176-5070 and which is available via NCBI BioSample SAMN15816600. The GC content of the type genome is $54.91 \%$ and the genome length is 1.5

5926 Mbp.

Description of Candidatus Limosilactobacillus intestinipullorum sp. nov.

5930

Candidatus Limosilactobacillus intestinipullorum (in.tes.ti.ni.pul.lo'rum. L. neut. n. intestinum

5931

5932 gut; L. masc. n. pullus a young chicken; N.L. gen. n. intestinipullorum of the gut of young chickens)

5933

5934

5935

5936

5937

5938

5939

5940

A bacterial species identified by metagenomic analyses. This species includes all bacteria with genomes that show $\geq 95 \%$ average nucleotide identity (ANI) to the type genome, which has been assigned the MAG ID ChiHecolR2B26-165 and which is available via NCBI BioSample SAMN15816601. The GC content of the type genome is $49.12 \%$ and the genome length is 1.6 Mbp.

\section{Description of Candidatus Limosilactobacillus merdavium sp. nov.}

5943

Candidatus Limosilactobacillus merdavium (merd.a'vi.um. L. fem. n. merda faeces; L. fem. n. 5944 avis bird; N.L. gen. n. merdavium of bird faeces)

A bacterial species identified by metagenomic analyses. This species includes all bacteria with genomes that show $\geq 95 \%$ average nucleotide identity (ANI) to the type genome, which has been assigned the MAG ID 876 and which is available via NCBI BioSample SAMN15816723. The $\mathrm{GC}$ content of the type genome is $39.60 \%$ and the genome length is $1.4 \mathrm{Mbp}$. 
Description of Candidatus Limosilactobacillus merdigallinarum sp. nov.

5953

Candidatus Limosilactobacillus merdigallinarum (mer.di.gal.li.na'rum. L. fem. n. merda faeces; L. fem. n. gallina hen; N.L. gen. n. merdigallinarum of hen faeces)

5954

5955

A bacterial species identified by metagenomic analyses. This species includes all bacteria with 5956

5957 genomes that show $\geq 95 \%$ average nucleotide identity (ANI) to the type genome, which has been assigned the MAG ID ChiSxjej3B15-572 and which is available via NCBI BioSample

5958

5959 SAMN15816736. The GC content of the type genome is $44.36 \%$ and the genome length is 1.4 Mbp.

5960

5961

5962

Description of Candidatus Limosilactobacillus merdipullorum sp. nov.

5963 Candidatus Limosilactobacillus merdipullorum (mer.di.pul.lo'rum. L. fem. n. merda faeces; L. masc. n. pullus a young chicken; N.L. gen. n. merdipullorum of the faeces of young chickens)

5965

5966

5967

5968

A bacterial species identified by metagenomic analyses. This species includes all bacteria with genomes that show $\geq 95 \%$ average nucleotide identity (ANI) to the type genome, which has been assigned the MAG ID ChiHejej3B27-2180 and which is available via NCBI BioSample

5969 SAMN15816756. The GC content of the type genome is $49.90 \%$ and the genome length is 1.3

5970

5971

5972

5973 Mbp.

\section{Description of Candidatus Limousia gen. nov.}

Candidatus Limousia (Lim.ou'si.a. L. masc. n. limus dung; Gr. fem. n. ousia an essence; N.L.

5975

5976

5977 fem. n. Limousia a microbe associated with faeces)

A bacterial genus identified by metagenomic analyses. The genus includes all bacteria with genomes that show $\geq 60 \%$ average amino acid identity (AAI) to the type genome from the type species Limousia pullorum. This is a name for the alphanumeric GTDB genus An172. This genus has been assigned by GTDB-Tk v1.3.0 working on GTDB Release 05-RS95 (Chaumeil et al., 2019; Parks et al., 2020) to the order Oscillospirales and to the family Acutalibacteraceae.

\section{Description of Candidatus Limousia pullorum sp. nov.}

A bacterial species identified by metagenomic analyses. This species includes all bacteria with genomes that show $\geq 95 \%$ average nucleotide identity (ANI) to the type genome, which has been assigned the MAG ID ChiGjej1B1-1684 and which is available via NCBI BioSample $\mathrm{GC}$ content of the type genome is $40.91 \%$ and the genome length is $1.7 \mathrm{Mbp}$.

\section{Description of Candidatus Luteimonas excrementigallinarum sp. nov.}


5995 Candidatus Luteimonas excrementigallinarum (ex.cre.men.ti.gal.li.na'rum. L. neut. n.

5996 excrementum excrement; L. fem. n. gallina hen; N.L. gen. n. excrementigallinarum of hen

5997 excrement)

5998

5999

A bacterial species identified by metagenomic analyses. This species includes all bacteria with

6000 genomes that show $\geq 95 \%$ average nucleotide identity (ANI) to the type genome, which has been

6001 assigned the MAG ID CHK165-14161 and which is available via NCBI BioSample

6002

6003 SAMN15816707. The GC content of the type genome is $68.39 \%$ and the genome length is 2.5 Mbp.

6004

6005

6006

Description of Candidatus Luteococcus avicola sp. nov.

6007

Candidatus Luteococcus avicola (a.vi'co.la. L. fem. n. avis bird; L. suff. -cola inhabitant of; N.L.

6008

n. avicola inhabitant of birds)

6009

6010

A bacterial species identified by metagenomic analyses. This species includes all bacteria with

6011

6012 genomes that show $\geq 95 \%$ average nucleotide identity (ANI) to the type genome, which has been

6013 assigned the MAG ID 4979 and which is available via NCBI BioSample SAMN15816867. This is a new name for the alphanumeric GTDB species sp002387005. The GC content of the type

6014 genome is $68.14 \%$ and the genome length is $2.9 \mathrm{Mbp}$.

6015

6016

6017

Description of Candidatus Mailhella excrementigallinarum sp. nov.

6018 Candidatus Mailhella excrementigallinarum (ex.cre.men.ti.gal.li.na'rum. L. neut. n. excrementum

6019 excrement; L. fem. n. gallina hen; N.L. gen. n. excrementigallinarum of hen excrement)

6020

6021

6022

6023

6024

6025

6026

6027

6028

6029

A bacterial species identified by metagenomic analyses. This species includes all bacteria with genomes that show $\geq 95 \%$ average nucleotide identity (ANI) to the type genome, which has been assigned the MAG ID 708 and which is available via NCBI BioSample SAMN15816871. This is a new name for the alphanumeric GTDB species sp003150275. The GC content of the type genome is $60.07 \%$ and the genome length is $3.0 \mathrm{Mbp}$.

6030

6031

6032

6033

\section{Description of Candidatus Mailhella merdavium sp. nov.}

Candidatus Mailhella merdavium (merd.a'vi.um. L. fem. n. merda faeces; L. fem. n. avis bird; N.L. gen. n. merdavium of bird faeces)

A bacterial species identified by metagenomic analyses. This species includes all bacteria with genomes that show $\geq 95 \%$ average nucleotide identity (ANI) to the type genome, which has been

6034 assigned the MAG ID ChiBcec6-11642 and which is available via NCBI BioSample

6035

6036

6037

6038

6039 SAMN15816648. The GC content of the type genome is $56.49 \%$ and the genome length is 2.7 Mbp.

Description of Candidatus Mailhella merdigallinarum sp. nov. 
6040 Candidatus Mailhella merdigallinarum (mer.di.gal.li.na'rum. L. fem. n. merda faeces; L. fem. n. 6041 gallina hen; N.L. gen. n. merdigallinarum of hen faeces)

6042

6043

6044

6045

A bacterial species identified by metagenomic analyses. This species includes all bacteria with genomes that show $\geq 95 \%$ average nucleotide identity (ANI) to the type genome, which has been assigned the MAG ID CHK186-16707 and which is available via NCBI BioSample

6046 SAMN15816842. This is a new name for the alphanumeric GTDB species sp900541395. The

6047

6048

6049

6050

Description of Candidatus Massiliomicrobiota merdigallinarum sp. nov.

6051 Candidatus Massiliomicrobiota merdigallinarum (mer.di.gal.li.na'rum. L. fem. n. merda faeces;

6052

L. fem. n. gallina hen; N.L. gen. n. merdigallinarum of hen faeces)

6053

6054

A bacterial species identified by metagenomic analyses. This species includes all bacteria with

6055 genomes that show $\geq 95 \%$ average nucleotide identity (ANI) to the type genome, which has been

6056 assigned the MAG ID CHK183-8118 and which is available via NCBI BioSample

6057 SAMN15816832. This is a new name for the alphanumeric GTDB species sp002160815. The

6058 $\mathrm{GC}$ content of the type genome is $31.33 \%$ and the genome length is $2.4 \mathrm{Mbp}$.

6059

6060

6061

\section{Description of Candidatus Mediterraneibacter avicola sp. nov.}

6062 Candidatus Mediterraneibacter avicola (a.vi'co.la. L. fem. n. avis bird; L. suff. -cola inhabitant

6063 of; N.L. n. avicola inhabitant of birds)

6064

6065

A bacterial species identified by metagenomic analyses. This species includes all bacteria with

6066

6067 genomes that show $\geq 95 \%$ average nucleotide identity (ANI) to the type genome, which has been assigned the MAG ID ChiGjej3B3-8055 and which is available via NCBI BioSample

6068 SAMN15816612. The GC content of the type genome is $48.65 \%$ and the genome length is 2.4

6069 Mbp.

6070

6071

6072

Description of Candidatus Mediterraneibacter caccavium sp. nov.

6073

Candidatus Mediterraneibacter caccavium (cacc.a'vi.um. Gr. fem. n. kakke faeces; L. fem. n. avis

6074 bird; N.L. gen. n. caccavium of bird faeces)

6075

6076

6077

6078

A bacterial species identified by metagenomic analyses. This species includes all bacteria with genomes that show $\geq 95 \%$ average nucleotide identity (ANI) to the type genome, which has been assigned the MAG ID ChiSjej5B23-15282 and which is available via NCBI BioSample

6079 SAMN15816861. This is a new name for the alphanumeric GTDB species sp002161355. The

6080

6081

6082

6083 $\mathrm{GC}$ content of the type genome is $51.40 \%$ and the genome length is $2.6 \mathrm{Mbp}$.

6084

\section{Description of Candidatus Mediterraneibacter caccogallinarum sp. nov.}

6085

Candidatus Mediterraneibacter caccogallinarum (cac.co.gal.li.na'rum. Gr. fem. n. kakke faeces; L. fem. n. gallina hen; N.L. gen. n. caccogallinarum of hen faeces) 
6086

6087

6088

6089

6090

6091

6092

6093

6094

6095

6096

6097

6098

6099

6100

6101

6102

6103

6104

6105

6106

6107

6108

6109

6110

6111

6112

6113

6114

6115

6116

6117

6118

6119

6120

6121

6122

6123

6124

6125

6126

6127

6128

6129

6130

6131

A bacterial species identified by metagenomic analyses. This species includes all bacteria with genomes that show $\geq 95 \%$ average nucleotide identity (ANI) to the type genome, which has been assigned the MAG ID ChiHcolR18-251 and which is available via NCBI BioSample

SAMN15816801. This is a new name for the alphanumeric GTDB species sp002314255. The $\mathrm{GC}$ content of the type genome is $50.75 \%$ and the genome length is $2.6 \mathrm{Mbp}$.

\section{Description of Candidatus Mediterraneibacter colneyensis sp. nov.}

Candidatus Mediterraneibacter colneyensis (col.ney.en'sis. N.L. fem. adj. colneyensis pertaining to Colney, the Norfolk village which is home to the Quadram Institute where the species was first described)

A bacterial species identified by metagenomic analyses. This species includes all bacteria with genomes that show $\geq 95 \%$ average nucleotide identity (ANI) to the type genome, which has been assigned the MAG ID ChiGjej5B5-19924 and which is available via NCBI BioSample SAMN15816732. The GC content of the type genome is $50.96 \%$ and the genome length is 1.9 Mbp.

\section{Description of Candidatus Mediterraneibacter cottocaccae sp. nov.}

Candidatus Mediterraneibacter cottocaccae (cot.to.cac'cae. Gr. masc. n. kottos chicken Gr. fem. n. kakke faeces; N.L. gen. n. cottocaccae of chicken faeces)

A bacterial species identified by metagenomic analyses. This species includes all bacteria with genomes that show $\geq 95 \%$ average nucleotide identity (ANI) to the type genome, which has been assigned the MAG ID CHK192-87 and which is available via NCBI BioSample SAMN15816835. This is a new name for the alphanumeric GTDB species sp002160525. The $\mathrm{GC}$ content of the type genome is $50.07 \%$ and the genome length is $4.0 \mathrm{Mbp}$.

\section{Description of Candidatus Mediterraneibacter excrementavium sp. nov.}

Candidatus Mediterraneibacter excrementavium (ex.cre.ment.a'vi.um. L. neut. n. excrementum excrement; L. fem. n. avis bird; N.L. gen. n. excrementavium of bird excrement)

A bacterial species identified by metagenomic analyses. This species includes all bacteria with genomes that show $\geq 95 \%$ average nucleotide identity (ANI) to the type genome, which has been assigned the MAG ID ChiGjej2B2-38138 and which is available via NCBI BioSample SAMN15816630. The GC content of the type genome is $51.42 \%$ and the genome length is 2.1 Mbp.

\section{Description of Candidatus Mediterraneibacter excrementigallinarum sp. nov.}

Candidatus Mediterraneibacter excrementigallinarum (ex.cre.men.ti.gal.li.na'rum. L. neut. n. excrementum excrement; L. fem. n. gallina hen; N.L. gen. n. excrementigallinarum of hen excrement) 
6132

6133

6134

6135

6136

6137

6138

6139

6140

6141

6142

6143

6144

6145

6146

6147

6148

6149

6150

6151

6152

6153

6154

6155

6156

6157

6158

6159

6160

6161

6162

6163

6164

6165

6166

6167

6168

6169

6170

6171

6172

6173

6174

6175

6176

6177

A bacterial species identified by metagenomic analyses. This species includes all bacteria with genomes that show $\geq 95 \%$ average nucleotide identity (ANI) to the type genome, which has been assigned the MAG ID CHK143-6153 and which is available via NCBI BioSample SAMN15816575. The GC content of the type genome is $48.97 \%$ and the genome length is 3.1 Mbp.

\section{Description of Candidatus Mediterraneibacter excrementipullorum sp. nov.} Candidatus Mediterraneibacter excrementipullorum (ex.cre.men.ti.pul.lo'rum. L. neut. n. excrementum excrement; L. masc. n. pullus a young chicken; N.L. gen. n. excrementipullorum of young chicken excrement)

A bacterial species identified by metagenomic analyses. This species includes all bacteria with genomes that show $\geq 95 \%$ average nucleotide identity (ANI) to the type genome, which has been assigned the MAG ID ChiSjej6B24-3024 and which is available via NCBI BioSample SAMN15816810. This is a new name for the alphanumeric GTDB species sp9005552. The GC content of the type genome is $48.88 \%$ and the genome length is $2.4 \mathrm{Mbp}$.

\section{Description of Candidatus Mediterraneibacter faecavium sp. nov.} Candidatus Mediterraneibacter faecavium (faec.a'vi.um. L. fem. n. faex, faecis excrement; L. fem. n. avis bird; N.L. gen. n. faecavium of bird faeces)

A bacterial species identified by metagenomic analyses. This species includes all bacteria with genomes that show $\geq 95 \%$ average nucleotide identity (ANI) to the type genome, which has been assigned the MAG ID CHK196-7946 and which is available via NCBI BioSample SAMN15816577. The GC content of the type genome is $49.45 \%$ and the genome length is 2.8 Mbp.

\section{Description of Candidatus Mediterraneibacter faecigallinarum sp. nov.} Candidatus Mediterraneibacter faecigallinarum (fae.ci.gal.li.na'rum. L. fem. n. faex, faecis excrement; L. fem. n. gallina hen; N.L. gen. n. faecigallinarum of hen faeces)

A bacterial species identified by metagenomic analyses. This species includes all bacteria with genomes that show $\geq 95 \%$ average nucleotide identity (ANI) to the type genome, which has been assigned the MAG ID ChiGjej1B1-1692 and which is available via NCBI BioSample SAMN15816637. The GC content of the type genome is $51.63 \%$ and the genome length is 2.6 Mbp.

\section{Description of Candidatus Mediterraneibacter faecipullorum sp. nov.}

Candidatus Mediterraneibacter faecipullorum (fae.ci.pul.lo'rum. L. fem. n. faex, faecis excrement; L. masc. n. pullus a young chicken; N.L. gen. n. faecipullorum of young chicken faeces)

Peer) reviewing PDF | (2020:12:55980:1:1:NEW 13 Jan 2021) 
6178

6179

6180

6181

6182

6183

6184

6185

6186

6187

6188

6189

6190

6191

6192

6193

6194

6195

6196

6197

6198

6199

6200

6201

6202

6203

6204

6205

6206

6207

6208

6209

6210

6211

6212

6213

6214

6215

6216

6217

6218

6219

6220

6221

6222
A bacterial species identified by metagenomic analyses. This species includes all bacteria with genomes that show $\geq 95 \%$ average nucleotide identity (ANI) to the type genome, which has been assigned the MAG ID ChiW19-954 and which is available via NCBI BioSample

SAMN15816638. The GC content of the type genome is $47.71 \%$ and the genome length is 2.7 Mbp.

\section{Description of Candidatus Mediterraneibacter gallistercoris sp. nov.}

Candidatus Mediterraneibacter gallistercoris (gal.li.ster'co.ris. L. masc. n. gallus chicken; L. neut. n. stercus dung; N.L. gen. n. gallistercoris of chicken faeces)

A bacterial species identified by metagenomic analyses. This species includes all bacteria with genomes that show $\geq 95 \%$ average nucleotide identity (ANI) to the type genome, which has been assigned the MAG ID CHK165-2605 and which is available via NCBI BioSample SAMN15816636. The GC content of the type genome is $47.20 \%$ and the genome length is 2.5 Mbp.

\section{Description of Candidatus Mediterraneibacter guildfordensis sp. nov.}

Candidatus Mediterraneibacter guildfordensis (guild.ford.en'sis. N.L. fem. adj. guildfordensis pertaining to Guildford, English town that is home to the University of Surrey)

A bacterial species identified by metagenomic analyses. This species includes all bacteria with genomes that show $\geq 95 \%$ average nucleotide identity (ANI) to the type genome, which has been assigned the MAG ID ChiHcec3-18395 and which is available via NCBI BioSample

SAMN15816784. The GC content of the type genome is $52.47 \%$ and the genome length is 2.2 Mbp.

\section{Description of Candidatus Mediterraneibacter intestinavium sp. nov.}

Candidatus Mediterraneibacter intestinavium (in.tes.tin.a'vi.um. L. neut. n. intestinum gut; L. fem. n. avis bird; N.L. gen. n. intestinavium of the gut of birds)

A bacterial species identified by metagenomic analyses. This species includes all bacteria with genomes that show $\geq 95 \%$ average nucleotide identity (ANI) to the type genome, which has been assigned the MAG ID ChiBcec12-2655 and which is available via NCBI BioSample SAMN15816591. The GC content of the type genome is $50.53 \%$ and the genome length is 2.9 Mbp.

\section{Description of Candidatus Mediterraneibacter intestinigallinarum sp. nov.}

Candidatus Mediterraneibacter intestinigallinarum (in.tes.ti.ni.gal.li.na'rum. L. neut. n. intestinum gut; L. fem. n. gallina hen; N.L. gen. n. intestinigallinarum of the gut of the hens) 
6223 A bacterial species identified by metagenomic analyses. This species includes all bacteria with 6224 genomes that show $\geq 95 \%$ average nucleotide identity (ANI) to the type genome, which has been 6225 assigned the MAG ID ChiBcec15-2237 and which is available via NCBI BioSample 6226 SAMN15816645. The GC content of the type genome is $46.81 \%$ and the genome length is 3.1 6227 Mbp.

6228

6229

6230

6231

6232

6233

6234

6235

6236

6237

6238

6239

6240

6241

6242

6243

6244

6245

6246

6247

6248

6249

6250

6251

6252

6253

6254

6255

6256

6257

6258

6259

6260

6261

6262

Description of Candidatus Mediterraneibacter intestinipullorum sp. nov.

Candidatus Mediterraneibacter intestinipullorum (in.tes.ti.ni.pul.lo'rum. L. neut. n. intestinum gut; L. masc. n. pullus a young chicken; N.L. gen. n. intestinipullorum of the gut of young chickens)

A bacterial species identified by metagenomic analyses. This species includes all bacteria with genomes that show $\geq 95 \%$ average nucleotide identity (ANI) to the type genome, which has been assigned the MAG ID CHK161-4361 and which is available via NCBI BioSample SAMN15816656. The GC content of the type genome is $49.75 \%$ and the genome length is 2.5 Mbp.

\section{Description of Candidatus Mediterraneibacter merdavium sp. nov.}

Candidatus Mediterraneibacter merdavium (merd.a'vi.um. L. fem. n. merda faeces; L. fem. n. avis bird; N.L. gen. n. merdavium of bird faeces)

A bacterial species identified by metagenomic analyses. This species includes all bacteria with genomes that show $\geq 95 \%$ average nucleotide identity (ANI) to the type genome, which has been assigned the MAG ID ChiBcolR7-8672 and which is available via NCBI BioSample SAMN15816660. The GC content of the type genome is $49.88 \%$ and the genome length is 2.6 Mbp.

\section{Description of Candidatus Mediterraneibacter merdigallinarum sp. nov.}

Candidatus Mediterraneibacter merdigallinarum (mer.di.gal.li.na'rum. L. fem. n. merda faeces; L. fem. n. gallina hen; N.L. gen. n. merdigallinarum of hen faeces)

A bacterial species identified by metagenomic analyses. This species includes all bacteria with genomes that show $\geq 95 \%$ average nucleotide identity (ANI) to the type genome, which has been assigned the MAG ID ChiW16-1363 and which is available via NCBI BioSample SAMN15816674. The GC content of the type genome is $46.95 \%$ and the genome length is 2.4 Mbp.

6263

6264

6265

Description of Candidatus Mediterraneibacter merdipullorum sp. nov.

6266

6267

Candidatus Mediterraneibacter merdipullorum (mer.di.pul.lo'rum. L. fem. n. merda faeces; L. masc. n. pullus a young chicken; N.L. gen. n. merdipullorum of the faeces of young chickens) 
6268 A bacterial species identified by metagenomic analyses. This species includes all bacteria with 6269 genomes that show $\geq 95 \%$ average nucleotide identity (ANI) to the type genome, which has been 6270 assigned the MAG ID ChiSxjej4B16-6421 and which is available via NCBI BioSample

6271 SAMN15816679. The GC content of the type genome is $54.17 \%$ and the genome length is 2.2

6272 Mbp.

6273

6274

6275

6276

6277

6278

6279

6280

6281

6282

6283

6284

6285

6286

6287

6288

6289

6290

6291

6292

6293

6294

6295

6296

6297

6298

Description of Candidatus Mediterraneibacter norfolkensis sp. nov.

Candidatus Mediterraneibacter norfolkensis (nor.folk.en'sis. N.L. fem. adj. norfolkensis pertaining to the English county of Norfolk)

A bacterial species identified by metagenomic analyses. This species includes all bacteria with genomes that show $\geq 95 \%$ average nucleotide identity (ANI) to the type genome, which has been assigned the MAG ID ChiW9-3490 and which is available via NCBI BioSample

SAMN15816789. The GC content of the type genome is $48.85 \%$ and the genome length is 3.5 Mbp.

Description of Candidatus Mediterraneibacter norwichensis sp. nov.

Candidatus Mediterraneibacter norwichensis (nor.wich.en'sis. N.L. fem. adj. norwichensis

pertaining to English city of Norwich)

A bacterial species identified by metagenomic analyses. This species includes all bacteria with genomes that show $\geq 95 \%$ average nucleotide identity (ANI) to the type genome, which has been assigned the MAG ID CHK180-4461 and which is available via NCBI BioSample SAMN15816628. The GC content of the type genome is $47.61 \%$ and the genome length is 2.6 Mbp.

\section{Description of Candidatus Mediterraneibacter ornithocaccae sp. nov.}

Candidatus Mediterraneibacter ornithocaccae (or.ni.tho.cac'cae. Gr. masc. or fem. n. ornis,

6299

ornithos bird Gr. fem. n. kakke faeces; N.L. gen. n. ornithocaccae of bird faeces)

6300

6301

A bacterial species identified by metagenomic analyses. This species includes all bacteria with

6302 genomes that show $\geq 95 \%$ average nucleotide identity (ANI) to the type genome, which has been

6303

6304 assigned the MAG ID ChiGjej1B1-20579 and which is available via NCBI BioSample

6305 SAMN15816839. This is a new name for the alphanumeric GTDB species sp002159505. The

6306 $\mathrm{GC}$ content of the type genome is $47.31 \%$ and the genome length is $2.6 \mathrm{Mbp}$.

6307

6308

Description of Candidatus Mediterraneibacter pullicola sp. nov.

6309 Candidatus Mediterraneibacter pullicola (pul.li'co.la. L. masc. n. pullus a young chicken; L. suff.

6310 -cola inhabitant of; N.L. n. pullicola an inhabitant of young chickens)

6311

6312 A bacterial species identified by metagenomic analyses. This species includes all bacteria with

6313 genomes that show $\geq 95 \%$ average nucleotide identity (ANI) to the type genome, which has been

Peer) reviewing PDF | (2020:12:55980:1:1:NEW 13 Jan 2021) 
6314 assigned the MAG ID ChiSjej2B20-11307 and which is available via NCBI BioSample

6315 SAMN15816678. The GC content of the type genome is $47.35 \%$ and the genome length is 2.1

6316 Mbp.

6317

6318

6319

Description of Candidatus Mediterraneibacter pullistercoris sp. nov.

6320 Candidatus Mediterraneibacter pullistercoris (pul.li.ster'co.ris. L. masc. n. pullus a young

6321 chicken; L. neut. n. stercus dung; N.L. gen. n. pullistercoris of young chicken faeces)

6322

6323

6324

6325

6326

6327

6328

6329

6330

A bacterial species identified by metagenomic analyses. This species includes all bacteria with genomes that show $\geq 95 \%$ average nucleotide identity (ANI) to the type genome, which has been assigned the MAG ID ChiHjej8B7-7219 and which is available via NCBI BioSample

SAMN15816602. The GC content of the type genome is $48.73 \%$ and the genome length is 2.3 Mbp.

6331

6332

6333

6334

6335

6336

6337

6338

\section{Description of Candidatus Mediterraneibacter quadrami sp. nov.}

Candidatus Mediterraneibacter quadrami (quad.ra'mi. N.L. gen. n. quadrami of the Quadram Institute)

A bacterial species identified by metagenomic analyses. This species includes all bacteria with genomes that show $\geq 95 \%$ average nucleotide identity (ANI) to the type genome, which has been assigned the MAG ID ChiBcec15-3976 and which is available via NCBI BioSample SAMN15816790. The GC content of the type genome is $52.68 \%$ and the genome length is 2.0 Mbp.

6339

6340

6341

Description of Candidatus Mediterraneibacter stercoravium sp. nov.

6342 Candidatus Mediterraneibacter stercoravium (ster.cor.a'vi.um. L. neut. n. stercus dung; L. fem.

6343 n. avis bird; N.L. gen. n. stercoravium of bird faeces)

6344

6345

6346

6347

6348

6349

6350

6351

6352

6353

Description of Candidatus Mediterraneibacter stercorigallinarum sp. nov.

6354

A bacterial species identified by metagenomic analyses. This species includes all bacteria with genomes that show $\geq 95 \%$ average nucleotide identity (ANI) to the type genome, which has been assigned the MAG ID CHK196-3914 and which is available via NCBI BioSample

SAMN15816603. The GC content of the type genome is $48.72 \%$ and the genome length is 2.5 Mbp.

6355

6356

6357

6358 Candidatus Mediterraneibacter stercorigallinarum (ster.co.ri.gal.li.na'rum. L. neut. n. stercus dung; L. fem. n. gallina hen; N.L. gen. n. stercorigallinarum of hen faeces)

A bacterial species identified by metagenomic analyses. This species includes all bacteria with genomes that show $\geq 95 \%$ average nucleotide identity (ANI) to the type genome, which has been assigned the MAG ID ChiGjej1B1-13045 and which is available via NCBI BioSample 
6359 SAMN15816697. The GC content of the type genome is $50.04 \%$ and the genome length is 2.3

$6360 \mathrm{Mbp}$.

6361

6362

6363

Description of Candidatus Mediterraneibacter stercoripullorum sp. nov.

6364

Candidatus Mediterraneibacter stercoripullorum (ster.co.ri.pul.lo'rum. L. neut. n. stercus dung;

6365

L. masc. n. pullus a young chicken; N.L. gen. n. stercoripullorum of the faceces of young

6366

chickens)

6367

6368

A bacterial species identified by metagenomic analyses. This species includes all bacteria with

6369 genomes that show $\geq 95 \%$ average nucleotide identity (ANI) to the type genome, which has been

6370 assigned the MAG ID CHK195-1396 and which is available via NCBI BioSample

6371

6372

SAMN15816610. The GC content of the type genome is $48.12 \%$ and the genome length is 3.2 Mbp.

6373

6374

6375

\section{Description of Candidatus Mediterraneibacter surreyensis sp. nov.}

6376

Candidatus Mediterraneibacter surreyensis (sur.rey.en'sis. N.L. fem. adj. surreyensis pertaining

6377

to the English county of Surrey where the samples in the study were collected)

6378

6379

A bacterial species identified by metagenomic analyses. This species includes all bacteria with

6380

6381 genomes that show $\geq 95 \%$ average nucleotide identity (ANI) to the type genome, which has been assigned the MAG ID CHK177-12742 and which is available via NCBI BioSample

6382 SAMN15816593. The GC content of the type genome is $46.80 \%$ and the genome length is 2.9

$6383 \mathrm{Mbp}$.

6384

6385

6386

6387

Description of Candidatus Mediterraneibacter tabaqchaliae sp. nov.

Candidatus Mediterraneibacter tabaqchaliae (ta.baq.cha'li.ae. N.L. fem. gen. n. tabaqchaliae

6388

named in honour of British microbiologist Soad Tabaqchali)

6389

6390

A bacterial species identified by metagenomic analyses. This species includes all bacteria with

6391 genomes that show $\geq 95 \%$ average nucleotide identity (ANI) to the type genome, which has been

6392 assigned the MAG ID ChiGjej3B3-11674 and which is available via NCBI BioSample

6393 SAMN15816791. The GC content of the type genome is $51.91 \%$ and the genome length is 2.7

$6394 \mathrm{Mbp}$.

6395

6396

6397

\section{Description of Candidatus Mediterraneibacter vanvlietii sp. nov.}

6398

Candidatus Mediterraneibacter vanvlietii (van.vliet'i.i. N.L. gen. n. vanvlietii named in honour of

6399 Dutch microbiologist Arnoud van Vliet)

6400

6401

A bacterial species identified by metagenomic analyses. This species includes all bacteria with

6402 genomes that show $\geq 95 \%$ average nucleotide identity (ANI) to the type genome, which has been

6403 assigned the MAG ID ChiBcec1-362 and which is available via NCBI BioSample 
6404 SAMN15816623. The GC content of the type genome is $48.47 \%$ and the genome length is 3.0 $6405 \mathrm{Mbp}$.

6406

6407

6408

6409

Description of Candidatus Megamonas gallistercoris sp. nov.

6410 Candidatus Megamonas gallistercoris (gal.li.ster'co.ris. L. masc. n. gallus chicken; L. neut. n. stercus dung; N.L. gen. n. gallistercoris of chicken faeces)

6411

6412

A bacterial species identified by metagenomic analyses. This species includes all bacteria with

6413 genomes that show $\geq 95 \%$ average nucleotide identity (ANI) to the type genome, which has been

6414 assigned the MAG ID ChiGjej6B6-7947 and which is available via NCBI BioSample

6415 SAMN15816859. This is a new name for the alphanumeric GTDB species sp900554895. The

6416

6417

6418 $\mathrm{GC}$ content of the type genome is $40.34 \%$ and the genome length is $2.2 \mathrm{Mbp}$.

6420

\section{Description of Candidatus Merdenecus gen. nov.}

6421

Candidatus Merdenecus (Merd.en.e'cus. L. fem. n. merda dung; Gr. masc. enoikos inhabitant;

6422

6423

6424

N.L. masc. n. Merdenecus a microbe associated with faeces)

6425

A bacterial genus identified by metagenomic analyses. The genus includes all bacteria with genomes that show $\geq 60 \%$ average amino acid identity (AAI) to the type genome from the type species Merdenecus merdavium. This is a name for the alphanumeric GTDB genus MCWD5. This genus has been assigned by GTDB-Tk v1.3.0 working on GTDB Release 05-RS95 (Chaumeil et al., 2019; Parks et al., 2020) to the order Lachnospirales and to the family

6429 Lachnospiraceae.

6430

6431

Description of Candidatus Merdenecus merdavium sp. nov.

6432 Candidatus Merdenecus merdavium (pul.li'co.la. L. masc. n. pullus a young chicken; L. suff. 6433 cola inhabitant of; N.L. n. pullicola an inhabitant of young chickens)

6434

6435

A bacterial species identified by metagenomic analyses. This species includes all bacteria with 6436

6437 genomes that show $\geq 95 \%$ average nucleotide identity (ANI) to the type genome, which has been assigned the MAG ID CHK160-2840 and which is available via NCBI BioSample

6439 SAMN15817122. The GC content of the type genome is $35.46 \%$ and the genome length is 2.6

6440

6441

6442 Mbp.

Description of Candidatus Merdibacter merdavium sp. nov.

6444 Candidatus Merdibacter merdavium (merd.a'vi.um. L. fem. n. merda faeces; L. fem. n. avis bird; 6445 N.L. gen. n. merdavium of bird faeces)

6447

A bacterial species identified by metagenomic analyses. This species includes all bacteria with 6448 genomes that show $\geq 95 \%$ average nucleotide identity (ANI) to the type genome, which has been assigned the MAG ID CHK187-11901 and which is available via NCBI BioSample 
6449 SAMN15816582. The GC content of the type genome is $53.13 \%$ and the genome length is 2.1

$6450 \mathrm{Mbp}$.

6451

6452

6453

Description of Candidatus Merdibacter merdigallinarum sp. nov.

6454 Candidatus Merdibacter merdigallinarum (mer.di.gal.li.na'rum. L. fem. n. merda faeces; L. fem.

6455

6456

6457 n. gallina hen; N.L. gen. n. merdigallinarum of hen faeces)

6458

A bacterial species identified by metagenomic analyses. This species includes all bacteria with

6459 genomes that show $\geq 95 \%$ average nucleotide identity (ANI) to the type genome, which has been

6460 assigned the MAG ID ChiGjej6B6-453 and which is available via NCBI BioSample

6461 SAMN15816595. The GC content of the type genome is $53.92 \%$ and the genome length is 1.8

6462

6463

6464 Mbp.

6465

Description of Candidatus Merdibacter merdipullorum sp. nov.

6466

6467

6468 Candidatus Merdibacter merdipullorum (mer.di.pul.lo'rum. L. fem. n. merda faeces; L. masc. $\mathrm{n}$. pullus a young chicken; N.L. gen. n. merdipullorum of the faeces of young chickens)

6469

A bacterial species identified by metagenomic analyses. This species includes all bacteria with

6470 genomes that show $\geq 95 \%$ average nucleotide identity (ANI) to the type genome, which has been

6471 assigned the MAG ID ChiGjej1B1-19782 and which is available via NCBI BioSample

6472 SAMN15816850. This is a new name for the alphanumeric GTDB species sp900543035. The

6473 $\mathrm{GC}$ content of the type genome is $55.41 \%$ and the genome length is $1.9 \mathrm{Mbp}$.

6474

6475

Description of Candidatus Merdicola gen. nov.

6476 Candidatus Merdicola (Merd.i.co'la. L. fem. n. merda dung; L. suff. -cola inhabitant of; N.L.

6477 fem. n. Merdicola a microbe associated with faeces)

6478

6479

A bacterial genus identified by metagenomic analyses. The genus includes all bacteria with

6480 genomes that show $\geq 60 \%$ average amino acid identity (AAI) to the type genome from the type

6481 species Merdicola faecigallinarum. This is a name for the alphanumeric GTDB genus CAG-354.

6482 This genus has been assigned by GTDB-Tk v1.3.0 working on GTDB Release 05-RS95

6483 (Chaumeil et al., 2019; Parks et al., 2020) to the order TANB77 and to the family CAG-508.

6484

6485

6486

6487

\section{Description of Candidatus Merdicola faecigallinarum sp. nov.}

6488

Candidatus Merdicola faecigallinarum (fae.ci.gal.li.na'rum. L. fem. n. faex, faecis excrement; L. fem. n. gallina hen; N.L. gen. n. faecigallinarum of hen faeces)

6489

6490

6491

A bacterial species identified by metagenomic analyses. This species includes all bacteria with

6492

6493 genomes that show $\geq 95 \%$ average nucleotide identity (ANI) to the type genome, which has been assigned the MAG ID CHK195-15760 and which is available via NCBI BioSample

6494 Mbp. 
Description of Candidatus Merdimorpha gen. nov.

6498 Candidatus Merdimorpha (Merd.i.mor'pha. L. fem. n. merda dung; Gr. fem. n. morphe a form, shape; N.L. fem. n. Merdimorpha a microbe associated with faeces)

6500

6501

6502

6503

A bacterial genus identified by metagenomic analyses. The genus includes all bacteria with genomes that show $\geq 60 \%$ average amino acid identity (AAI) to the type genome from the type species Merdimorpha intestinavium. This is a name for the alphanumeric GTDB genus UBA1820. This genus has been assigned by GTDB-Tk v1.3.0 working on GTDB Release 05RS95 (Chaumeil et al., 2019; Parks et al., 2020) to the order Flavobacteriales and to the family

\section{Description of Candidatus Merdimorpha intestinavium sp. nov.}

6510 Candidatus Merdimorpha intestinavium (in.tes.tin.a'vi.um. L. neut. n. intestinum gut; L. fem. $\mathrm{n}$. 6511 avis bird; N.L. gen. n. intestinavium of the gut of birds)

6512

6513

6514

6515

6516

6517

6518

6519

6520

A bacterial species identified by metagenomic analyses. This species includes all bacteria with genomes that show $\geq 95 \%$ average nucleotide identity (ANI) to the type genome, which has been assigned the MAG ID CHK1-7158 and which is available via NCBI BioSample SAMN15817210. This is a new name for the alphanumeric GTDB species sp002314265. The $\mathrm{GC}$ content of the type genome is $56.50 \%$ and the genome length is $1.8 \mathrm{Mbp}$.

6521

6522

6523

6524

6525

6526

6527

6528

6529

6530

\section{Description of Candidatus Merdimorpha stercoravium sp. nov.}

Candidatus Merdimorpha stercoravium (ster.cor.a'vi.um. L. neut. n. stercus dung; L. fem. n. avis bird; N.L. gen. n. stercoravium of bird faeces)

A bacterial species identified by metagenomic analyses. This species includes all bacteria with genomes that show $\geq 95 \%$ average nucleotide identity (ANI) to the type genome, which has been assigned the MAG ID 1383 and which is available via NCBI BioSample SAMN15817125. The $\mathrm{GC}$ content of the type genome is $57.82 \%$ and the genome length is $1.7 \mathrm{Mbp}$.

6531

6532

6533

6534

6535

6536

6537

6538

6539

\section{Description of Candidatus Merdiplasma gen. nov.}

Candidatus Merdiplasma (Merd.i.plas'ma. L. fem. n. merda dung; Gr. neut. n. plasma a form; N.L. neut. n. Merdiplasma a microbe associated with faeces)

A bacterial genus identified by metagenomic analyses. The genus includes all bacteria with genomes that show $\geq 60 \%$ average amino acid identity (AAI) to the type genome from the type species Merdiplasma excrementigallinarum. This is a name for the alphanumeric GTDB genus UBA2856. This genus has been assigned by GTDB-Tk v1.3.0 working on GTDB Release 05RS95 (Chaumeil et al., 2019; Parks et al., 2020) to the order Lachnospirales and to the family

6540 
6542 Description of Candidatus Merdiplasma excrementigallinarum sp. nov.

6543 Candidatus Merdiplasma excrementigallinarum (ex.cre.men.ti.gal.li.na'rum. L. neut. n.

6544 excrementum excrement; L. fem. n. gallina hen; N.L. gen. n. excrementigallinarum of hen

6545 excrement)

6546

6547 A bacterial species identified by metagenomic analyses. This species includes all bacteria with 6548 genomes that show $\geq 95 \%$ average nucleotide identity (ANI) to the type genome, which has been 6549 assigned the MAG ID ChiBcec6-7307 and which is available via NCBI BioSample

6550 SAMN15817161. The GC content of the type genome is $52.97 \%$ and the genome length is 2.4

$6551 \mathrm{Mbp}$.

6552

6553

6554

6555

Description of Candidatus Merdisoma gen. nov.

Candidatus Merdisoma (Merd.i.so'ma. L. fem. n. merda dung; Gr. neut. n. soma a body; N.L.

6556

neut. n. Merdisoma a microbe associated with faeces)

6557

6558

A bacterial genus identified by metagenomic analyses. The genus includes all bacteria with

6559 genomes that show $\geq 60 \%$ average amino acid identity (AAI) to the type genome from the type

6560 species Merdisoma merdipullorum. This is a name for the alphanumeric GTDB genus GCA-

6561 900066575. This genus has been assigned by GTDB-Tk v1.3.0 working on GTDB Release 05-

6562

6563 RS95 (Chaumeil et al., 2019; Parks et al., 2020) to the order Lachnospirales and to the family

6564

6565

Description of Candidatus Merdisoma faecalis sp. nov.

6567

Candidatus Merdisoma faecalis (fae.ca'lis. L. fem. adj. faecalis of faeces)

6568

6569

A bacterial species identified by metagenomic analyses. This species includes all bacteria with

6570 genomes that show $\geq 95 \%$ average nucleotide identity (ANI) to the type genome, which has been

6571 assigned the MAG ID ChiBcolR2-1241 and which is available via NCBI BioSample

6572 SAMN15817219. This is a new name for the alphanumeric GTDB species sp002160765. The

6573 $\mathrm{GC}$ content of the type genome is $51.56 \%$ and the genome length is $2.7 \mathrm{Mbp}$.

6574

6575

6576

\section{Description of Candidatus Merdisoma merdipullorum sp. nov.}

6577 Candidatus Merdisoma merdipullorum (mer.di.pul.lo'rum. L. fem. n. merda faeces; L. masc. n. pullus a young chicken; N.L. gen. n. merdipullorum of the faeces of young chickens)

A bacterial species identified by metagenomic analyses. This species includes all bacteria with genomes that show $\geq 95 \%$ average nucleotide identity (ANI) to the type genome, which has been assigned the MAG ID CHK197-19677 and which is available via NCBI BioSample

6583 SAMN15817042. The GC content of the type genome is 50.06\% and the genome length is 2.9

$6584 \mathrm{Mbp}$.

6585

6586 
6587 Description of Candidatus Merdivicinus gen. nov.

6588 Candidatus Merdivicinus (Merd.ivic.in'us. L. fem. n. merda dung; L. masc. n. vicinus a

6589

6590

6591 neighbour; N.L. masc. n. Merdivicinus a microbe associated with faeces)

6592

A bacterial genus identified by metagenomic analyses. The genus includes all bacteria with

6593 genomes that show $\geq 60 \%$ average amino acid identity (AAI) to the type genome from the type

6594 species Merdivicinus faecavium. This is a name for the alphanumeric GTDB genus UMGS1826.

6595 This genus has been assigned by GTDB-Tk v1.3.0 working on GTDB Release 05-RS95

6596 (Chaumeil et al., 2019; Parks et al., 2020) to the order Oscillospirales and to the family

6597 Ruminococcaceae.

6598

6599

Description of Candidatus Merdivicinus excrementipullorum sp. nov.

6600 Candidatus Merdivicinus excrementipullorum (ex.cre.men.ti.pul.lo'rum. L. neut. n. excrementum

6601 excrement; L. masc. n. pullus a young chicken; N.L. gen. n. excrementipullorum of young

6602 chicken excrement)

6603

6604

A bacterial species identified by metagenomic analyses. This species includes all bacteria with

6605 genomes that show $\geq 95 \%$ average nucleotide identity (ANI) to the type genome, which has been

6606 assigned the MAG ID CHK199-13235 and which is available via NCBI BioSample

6607 SAMN15817032. The GC content of the type genome is $54.67 \%$ and the genome length is 2.5

$6608 \mathrm{Mbp}$.

6609

6610

6611

\section{Description of Candidatus Merdivicinus faecavium sp. nov.}

6612 Candidatus Merdivicinus faecavium (faec.a'vi.um. L. fem. n. faex, faecis excrement; L. fem. n.

6613

6614

6615 avis bird; N.L. gen. n. faecavium of bird faeces)

6616

A bacterial species identified by metagenomic analyses. This species includes all bacteria with

6617 genomes that show $\geq 95 \%$ average nucleotide identity (ANI) to the type genome, which has been

6618 assigned the MAG ID CHK186-19003 and which is available via NCBI BioSample

6619 SAMN15817036. The GC content of the type genome is $61.26 \%$ and the genome length is 2.5

6620 Mbp.

6621

6622

\section{Description of Candidatus Merdivicinus intestinavium sp. nov.}

6623 Candidatus Merdivicinus intestinavium (in.tes.tin.a'vi.um. L. neut. n. intestinum gut; L. fem. n.

6624

6625

6626 avis bird; N.L. gen. n. intestinavium of the gut of birds)

6627

A bacterial species identified by metagenomic analyses. This species includes all bacteria with genomes that show $\geq 95 \%$ average nucleotide identity (ANI) to the type genome, which has been

6628 assigned the MAG ID CHK188-1901 and which is available via NCBI BioSample

6629 SAMN15817003. The GC content of the type genome is $59.41 \%$ and the genome length is 2.3

6630 Mbp.

6631

6632 
6633 Description of Candidatus Merdivicinus intestinigallinarum sp. nov.

6634 Candidatus Merdivicinus intestinigallinarum (in.tes.ti.ni.gal.li.na'rum. L. neut. n. intestinum gut;

6635

6636

6637

L. fem. n. gallina hen; N.L. gen. n. intestinigallinarum of the gut of the hens)

6638

A bacterial species identified by metagenomic analyses. This species includes all bacteria with

6639 genomes that show $\geq 95 \%$ average nucleotide identity (ANI) to the type genome, which has been assigned the MAG ID ChiBcec18-2170 and which is available via NCBI BioSample

6640 SAMN15817163. The GC content of the type genome is $56.27 \%$ and the genome length is 2.5

$6641 \mathrm{Mbp}$.

6642

6643

6644

Description of Candidatus Merdivivens gen. nov.

6645 Candidatus Merdivivens (Merd.i.viv'ens. L. fem. n. merda dung; N.L. pres. part. vivens living;

6646 N.L. fem. n. Merdivivens a microbe associated with faeces)

6647

6648

A bacterial genus identified by metagenomic analyses. The genus includes all bacteria with

6649

6650 genomes that show $\geq 60 \%$ average amino acid identity (AAI) to the type genome from the type

6651 species Merdivivens pullistercoris. This is a name for the alphanumeric GTDB genus UBA3382.

6652 This genus has been assigned by GTDB-Tk v1.3.0 working on GTDB Release 05-RS95

6653 (Chaumeil et al., 2019; Parks et al., 2020) to the order Bacteroidales and to the family UBA932.

6654

6655

Description of Candidatus Merdivivens faecigallinarum sp. nov.

6656 Candidatus Merdivivens faecigallinarum (fae.ci.gal.li.na'rum. L. fem. n. faex, faecis excrement;

6657 L. fem. n. gallina hen; N.L. gen. n. faecigallinarum of hen faeces)

6658

6659

A bacterial species identified by metagenomic analyses. This species includes all bacteria with

6660

6661 genomes that show $\geq 95 \%$ average nucleotide identity (ANI) to the type genome, which has been

6662 assigned the MAG ID B3-2255 and which is available via NCBI BioSample SAMN15817168.

6663 This is a new name for the alphanumeric GTDB species sp002159555. The GC content of the type genome is $49.87 \%$ and the genome length is $1.9 \mathrm{Mbp}$.

6664

6665

6666

6667

\section{Description of Candidatus Merdivivens pullicola sp. nov.}

6668

Candidatus Merdivivens pullicola (pul.li'co.la. L. masc. n. pullus a young chicken; L. suff. -cola

6669

6670 inhabitant of; N.L. n. pullicola an inhabitant of young chickens)

6671

6672

A bacterial species identified by metagenomic analyses. This species includes all bacteria with genomes that show $\geq 95 \%$ average nucleotide identity (ANI) to the type genome, which has been

6673 assigned the MAG ID B1-8020 and which is available via NCBI BioSample SAMN15817062.

6674

6675

6676

6677 The GC content of the type genome is $48.22 \%$ and the genome length is $2.0 \mathrm{Mbp}$.

6678

\section{Description of Candidatus Merdivivens pullistercoris sp. nov.}

Candidatus Merdivivens pullistercoris (pul.li.ster'co.ris. L. masc. n. pullus a young chicken; L. neut. n. stercus dung; N.L. gen. n. pullistercoris of young chicken faeces) 
6679

6680

6681

6682

6683

6684

6685

6686

6687

6688

6689

6690

6691

6692

6693

6694

6695

6696

6697

6698

6699

6700

6701

6702

6703

6704

6705

6706

6707

6708

6709

6710

6711

6712

6713

6714

6715

6716

6717

6718

6719

6720

6721

6722

6723

6724

A bacterial species identified by metagenomic analyses. This species includes all bacteria with genomes that show $\geq 95 \%$ average nucleotide identity (ANI) to the type genome, which has been assigned the MAG ID 10037 and which is available via NCBI BioSample SAMN15817074. The GC content of the type genome is $48.95 \%$ and the genome length is $2.1 \mathrm{Mbp}$.

\section{Description of Candidatus Merdousia gen. nov.}

Candidatus Merdousia (Merd.ou'si.a. L. fem. n. merda dung; Gr. fem. n. ousia an essence; N.L. fem. n. Merdousia a microbe associated with faeces)

A bacterial genus identified by metagenomic analyses. The genus includes all bacteria with genomes that show $\geq 60 \%$ average amino acid identity (AAI) to the type genome from the type species Merdousia gallistercoris. This is a name for the alphanumeric GTDB genus CAG-312. This genus has been assigned by GTDB-Tk v1.3.0 working on GTDB Release 05-RS95 (Chaumeil et al., 2019; Parks et al., 2020) to the order Opitutales and to the family CAG-312.

\section{Description of Candidatus Merdousia gallistercoris sp. nov.}

Candidatus Merdousia gallistercoris (gal.li.ster'co.ris. L. masc. $\mathrm{n}$ gallus chicken; L. neut. $\mathrm{n}$. stercus dung; N.L. gen. n. gallistercoris of chicken faeces)

A bacterial species identified by metagenomic analyses. This species includes all bacteria with genomes that show $\geq 95 \%$ average nucleotide identity (ANI) to the type genome, which has been assigned the MAG ID CHK197-16368 and which is available via NCBI BioSample SAMN15817207. This is a new name for the alphanumeric GTDB species sp900545715. The GC content of the type genome is $49.37 \%$ and the genome length is $2.4 \mathrm{Mbp}$.

\section{Description of Candidatus Methanocorpusculum faecipullorum sp. nov.} Candidatus Methanocorpusculum faecipullorum (fae.ci.pul.lo'rum. L. fem. n. faex, faecis excrement; L. masc. n. pullus a young chicken; N.L. gen. n. faecipullorum of young chicken faeces)

A bacterial species identified by metagenomic analyses. This species includes all bacteria with genomes that show $\geq 95 \%$ average nucleotide identity (ANI) to the type genome, which has been assigned the MAG ID E1-3281 and which is available via NCBI BioSample SAMN15816796. The GC content of the type genome is $50.72 \%$ and the genome length is $1.2 \mathrm{Mbp}$.

\section{Description of Candidatus Microbacterium pullistercoris sp. nov.}

Candidatus Microbacterium pullistercoris (pul.li.ster'co.ris. L. masc. n. pullus a young chicken; L. neut. n. stercus dung; N.L. gen. n. pullistercoris of young chicken faeces)

A bacterial species identified by metagenomic analyses. This species includes all bacteria with genomes that show $\geq 95 \%$ average nucleotide identity (ANI) to the type genome, which has been 
6725 assigned the MAG ID ChiGjej1B1-5908 and which is available via NCBI BioSample

6726 SAMN15816649. The GC content of the type genome is $68.74 \%$ and the genome length is 2.5

6727 Mbp.

6728

6729

6730

Description of Candidatus Microbacterium stercoravium sp. nov.

6731

Candidatus Microbacterium stercoravium (ster.cor.a'vi.um. L. neut. n. stercus dung; L. fem. $\mathrm{n}$.

6732

avis bird; N.L. gen. n. stercoravium of bird faeces)

6733

6734

A bacterial species identified by metagenomic analyses. This species includes all bacteria with

6735 genomes that show $\geq 95 \%$ average nucleotide identity (ANI) to the type genome, which has been

6736 assigned the MAG ID ChiHjej8B7-3636 and which is available via NCBI BioSample

6737 SAMN15816680. The GC content of the type genome is $69.44 \%$ and the genome length is 2.5

6738 Mbp.

6739

6740

6741

Description of Candidatus Monoglobus merdigallinarum sp. nov.

6742 Candidatus Monoglobus merdigallinarum (mer.di.gal.li.na'rum. L. fem. n. merda faeces; L. fem.

6743

6744

6745 n. gallina hen; N.L. gen. n. merdigallinarum of hen faeces)

6746

A bacterial species identified by metagenomic analyses. This species includes all bacteria with genomes that show $\geq 95 \%$ average nucleotide identity (ANI) to the type genome, which has been

6747

6748 assigned the MAG ID 5790 and which is available via NCBI BioSample SAMN15816780. The GC content of the type genome is $48.24 \%$ and the genome length is $1.5 \mathrm{Mbp}$.

6749

6750

6751

Description of Candidatus Mucispirillum faecigallinarum sp. nov.

6752

Candidatus Mucispirillum faecigallinarum (fae.ci.gal.li.na'rum. L. fem. n. faex, faecis

6753 excrement; L. fem. n. gallina hen; N.L. gen. n. faecigallinarum of hen faeces)

6754

6755

A bacterial species identified by metagenomic analyses. This species includes all bacteria with

6756

6757 genomes that show $\geq 95 \%$ average nucleotide identity (ANI) to the type genome, which has been assigned the MAG ID ChiW4-1371 and which is available via NCBI BioSample

6758

SAMN15816684. The GC content of the type genome is $31.75 \%$ and the genome length is 2.2

6759 Mbp.

6760

6761

6762

Description of Candidatus Negativibacillus faecipullorum sp. nov.

6763

Candidatus Negativibacillus faecipullorum (fae.ci.pul.lo'rum. L. fem. n. faex, faecis excrement;

6764

L. masc. n. pullus a young chicken; N.L. gen. n. faecipullorum of young chicken faeces)

6765

6766

6767

A bacterial species identified by metagenomic analyses. This species includes all bacteria with

6768 genomes that show $\geq 95 \%$ average nucleotide identity (ANI) to the type genome, which has been

6769 assigned the MAG ID ChiBcec6-1156 and which is available via NCBI BioSample

6770 SAMN15816879. This is a new name for the alphanumeric GTDB species sp900547455. The GC content of the type genome is $57.54 \%$ and the genome length is $2.0 \mathrm{Mbp}$. 
6771

6772

6773

6774

6775

6776

6777

6778

6779

6780

6781

6782

6783

6784

6785

6786

6787

6788

6789

6790

6791

6792

6793

6794

6795

6796

6797

6798

6799

6800

6801

6802

6803

6804

6805

6806

6807

6808

6809

6810

6811

6812

6813

6814

6815

6816

Description of Candidatus Nesterenkonia stercoripullorum sp. nov.

Candidatus Nesterenkonia stercoripullorum (ster.co.ri.pul.lo'rum. L. neut. n. stercus dung; L. masc. n. pullus a young chicken; N.L. gen. n. stercoripullorum of the faceces of young chickens)

A bacterial species identified by metagenomic analyses. This species includes all bacteria with genomes that show $\geq 95 \%$ average nucleotide identity (ANI) to the type genome, which has been assigned the MAG ID ChiHejej3B27-3195 and which is available via NCBI BioSample

SAMN15816751. The GC content of the type genome is $65.88 \%$ and the genome length is 2.6 Mbp.

\section{Description of Candidatus Niameybacter stercoravium sp. nov.}

Candidatus Niameybacter stercoravium (ster.cor.a'vi.um. L. neut. n. stercus dung; L. fem. n. avis bird; N.L. gen. n. stercoravium of bird faeces)

A bacterial species identified by metagenomic analyses. This species includes all bacteria with genomes that show $\geq 95 \%$ average nucleotide identity (ANI) to the type genome, which has been assigned the MAG ID 2467 and which is available via NCBI BioSample SAMN15816773. The $\mathrm{GC}$ content of the type genome is $35.14 \%$ and the genome length is $2.9 \mathrm{Mbp}$.

\section{Description of Candidatus Nocardiopsis merdipullorum sp. nov.}

Candidatus Nocardiopsis merdipullorum (mer.di.pul.lo'rum. L. fem. n. merda faeces; L. masc. n. pullus a young chicken; N.L. gen. n. merdipullorum of the faeces of young chickens)

A bacterial species identified by metagenomic analyses. This species includes all bacteria with genomes that show $\geq 95 \%$ average nucleotide identity (ANI) to the type genome, which has been assigned the MAG ID ChiHjej10B9-18110 and which is available via NCBI BioSample SAMN15816716. The GC content of the type genome is $65.81 \%$ and the genome length is 4.2 Mbp.

\section{Description of Candidatus Nosocomiicoccus stercorigallinarum sp. nov.}

Candidatus Nosocomiicoccus stercorigallinarum (ster.co.ri.gal.li.na'rum. L. neut. n. stercus dung; L. fem. n. gallina hen; N.L. gen. n. stercorigallinarum of hen faeces)

A bacterial species identified by metagenomic analyses. This species includes all bacteria with genomes that show $\geq 95 \%$ average nucleotide identity (ANI) to the type genome, which has been assigned the MAG ID CHK169-14505 and which is available via NCBI BioSample SAMN15816647. The GC content of the type genome is $34.64 \%$ and the genome length is 1.3 Mbp.

Description of Candidatus Oceanisphaera merdipullorum sp. nov.

Peer) reviewing PDF | (2020:12:55980:1:1:NEW 13 Jan 2021) 
6817 Candidatus Oceanisphaera merdipullorum (mer.di.pul.lo'rum. L. fem. n. merda faeces; L. masc. 6818 n. pullus a young chicken; N.L. gen. n. merdipullorum of the faeces of young chickens)

6819

6820

6821

6822

6823

6824

6825

6826

6827

6828

6829

6830

6831

6832

6833

6834

6835

6836

6837

6838

6839

6840

6841

6842

6843

6844

6845

6846

6847

6848

6849

6850

6851

6852

6853

6854

6855

6856

6857

6858

6859

6860

6861

6862

A bacterial species identified by metagenomic analyses. This species includes all bacteria with genomes that show $\geq 95 \%$ average nucleotide identity (ANI) to the type genome, which has been assigned the MAG ID 819 and which is available via NCBI BioSample SAMN15816797. The $\mathrm{GC}$ content of the type genome is $50.19 \%$ and the genome length is $2.9 \mathrm{Mbp}$.

\section{Description of Candidatus Odoribacter faecigallinarum sp. nov.}

Candidatus Odoribacter faecigallinarum (fae.ci.gal.li.na'rum. L. fem. n. faex, faecis excrement; L. fem. n. gallina hen; N.L. gen. n. faecigallinarum of hen faeces)

A bacterial species identified by metagenomic analyses. This species includes all bacteria with genomes that show $\geq 95 \%$ average nucleotide identity (ANI) to the type genome, which has been assigned the MAG ID 23274 and which is available via NCBI BioSample SAMN15816743. The $\mathrm{GC}$ content of the type genome is $48.17 \%$ and the genome length is $2.2 \mathrm{Mbp}$.

\section{Description of Candidatus Olsenella avicola sp. nov.}

Candidatus Olsenella avicola (a.vi'co.la. L. fem. n. avis bird; L. suff. -cola inhabitant of; N.L. n. avicola inhabitant of birds)

A bacterial species identified by metagenomic analyses. This species includes all bacteria with genomes that show $\geq 95 \%$ average nucleotide identity (ANI) to the type genome, which has been assigned the MAG ID CHK1-7693 and which is available via NCBI BioSample SAMN15816923. This is a new name for the alphanumeric GTDB species sp002159625. Although GTDB has assigned this species to the genus it calls Olsenella_E, this genus designation cannot be incorporated into a well-formed binomial, so in naming this species, we have used the current validly published name for the genus. The GC content of the type genome is $67.59 \%$ and the genome length is $2.2 \mathrm{Mbp}$.

\section{Description of Candidatus Olsenella avistercoris sp. nov.}

Candidatus Olsenella avistercoris (a.vi.ster'co.ris. L. fem. n. avis bird; L. neut. n. stercus dung; N.L. gen. n. avistercoris of bird faeces)

A bacterial species identified by metagenomic analyses. This species includes all bacteria with genomes that show $\geq 95 \%$ average nucleotide identity (ANI) to the type genome, which has been assigned the MAG ID CHK136-6238 and which is available via NCBI BioSample SAMN15816919. This is a new name for the alphanumeric GTDB species sp002160255. Although GTDB has assigned this species to the genus it calls Olsenella_E, this genus designation cannot be incorporated into a well-formed binomial, so in naming this species, we have used the current validly published name for the genus. The GC content of the type genome is $69.05 \%$ and the genome length is $2.0 \mathrm{Mbp}$. 
Description of Candidatus Olsenella excrementavium sp. nov.

6866 Candidatus Olsenella excrementavium (ex.cre.ment.a'vi.um. L. neut. n. excrementum excrement;

6867 L. fem. n. avis bird; N.L. gen. n. excrementavium of bird excrement)

6869

A bacterial species identified by metagenomic analyses. This species includes all bacteria with

6870 genomes that show $\geq 95 \%$ average nucleotide identity (ANI) to the type genome, which has been assigned the MAG ID ChiHjej10B9-743 and which is available via NCBI BioSample SAMN15816922. This is a new name for the alphanumeric GTDB species sp002305805. Although GTDB has assigned this species to the genus it calls Olsenella_E, this genus designation cannot be incorporated into a well-formed binomial, so in naming this species, we

6875 have used the current validly published name for the genus. The GC content of the type genome

6876

6877 is $66.50 \%$ and the genome length is $1.8 \mathrm{Mbp}$.

6879

\section{Description of Candidatus Olsenella excrementigallinarum sp. nov.}

6880

6881

6882

6883 Candidatus Olsenella excrementigallinarum (ex.cre.men.ti.gal.li.na'rum. L. neut. n. excrementum excrement; L. fem. n. gallina hen; N.L. gen. n. excrementigallinarum of hen excrement)

A bacterial species identified by metagenomic analyses. This species includes all bacteria with genomes that show $\geq 95 \%$ average nucleotide identity (ANI) to the type genome, which has been assigned the MAG ID ChiHjej12B11-23512 and which is available via NCBI BioSample SAMN15816920. This is a new name for the alphanumeric GTDB species sp900119915. Although GTDB has assigned this species to the genus it calls Olsenella_E, this genus designation cannot be incorporated into a well-formed binomial, so in naming this species, we have used the current validly published name for the genus. The GC content of the type genome is $68.67 \%$ and the genome length is $1.8 \mathrm{Mbp}$.

6890

6891

\section{Description of Candidatus Olsenella pullicola sp. nov.}

6893 Candidatus Olsenella pullicola (pul.li'co.la. L. masc. n. pullus a young chicken; L. suff. -cola 6894 inhabitant of; N.L. n. pullicola an inhabitant of young chickens)

6897

A bacterial species identified by metagenomic analyses. This species includes all bacteria with genomes that show $\geq 95 \%$ average nucleotide identity (ANI) to the type genome, which has been assigned the MAG ID ChiHecec1B25-7792 and which is available via NCBI BioSample SAMN15816895. Although GTDB has assigned this species to the genus it calls Olsenella_E, this genus designation cannot be incorporated into a well-formed binomial, so in naming this

6902 species, we have used the current validly published name for the genus. The GC content of the 6903 type genome is $65.74 \%$ and the genome length is $2.3 \mathrm{Mbp}$.

\section{Description of Candidatus Olsenella pullistercoris sp. nov.}

6907 Candidatus Olsenella pullistercoris (pul.li.ster'co.ris. L. masc. n. pullus a young chicken; L. neut. 6908 
6909 A bacterial species identified by metagenomic analyses. This species includes all bacteria with 6910 genomes that show $\geq 95 \%$ average nucleotide identity (ANI) to the type genome, which has been 6911 assigned the MAG ID ChiHjej12B11-14209 and which is available via NCBI BioSample

6912 SAMN15816899. Although GTDB has assigned this species to the genus it calls Olsenella_E, 6913 this genus designation cannot be incorporated into a well-formed binomial, so in naming this 6914 species, we have used the current validly published name for the genus. The GC content of the 6915 type genome is $67.21 \%$ and the genome length is $1.9 \mathrm{Mbp}$.

6916

6917

6918

\section{Description of Candidatus Olsenella stercoravium sp. nov.}

6919 Candidatus Olsenella stercoravium (ster.cor.a'vi.um. L. neut. n. stercus dung; L. fem. n. avis

6920

6921

6922

6923

6924 bird; N.L. gen. n. stercoravium of bird faeces)

A bacterial species identified by metagenomic analyses. This species includes all bacteria with genomes that show $\geq 95 \%$ average nucleotide identity (ANI) to the type genome, which has been assigned the MAG ID ChiHecolR3B27-1887 and which is available via NCBI BioSample

6925

6926

6927

6928

6929 SAMN15816902. Although GTDB has assigned this species to the genus it calls Olsenella_E, this genus designation cannot be incorporated into a well-formed binomial, so in naming this species, we have used the current validly published name for the genus. The GC content of the type genome is $67.26 \%$ and the genome length is $1.8 \mathrm{Mbp}$.

6930

6931

6932

Description of Candidatus Onthenecus gen. nov.

6933

Candidatus Onthenecus (Onthen.e'cus. Gr. masc. n. onthos dung; Gr. masc. enoikos inhabitant;

6934

6935

6936

6937

6938

6939 N.L. masc. n. Onthenecus a microbe associated with faeces)

A bacterial genus identified by metagenomic analyses. The genus includes all bacteria with genomes that show $\geq 60 \%$ average amino acid identity (AAI) to the type genome from the type species Onthenecus intestinigallinarum. This is a name for the alphanumeric GTDB genus OEMS01. This genus has been assigned by GTDB-Tk v1.3.0 working on GTDB Release 05RS95 (Chaumeil et al., 2019; Parks et al., 2020) to the order Christensenellales and to the family

6940

6941

6942

6943

6944 CAG-74.

6945

6946

6947

6948

6949

6950

6951

\section{Description of Candidatus Onthenecus intestinigallinarum sp. nov.}

Candidatus Onthenecus intestinigallinarum (in.tes.ti.ni.gal.li.na'rum. L. neut. n. intestinum gut; L. fem. n. gallina hen; N.L. gen. n. intestinigallinarum of the gut of the hens)

A bacterial species identified by metagenomic analyses. This species includes all bacteria with genomes that show $\geq 95 \%$ average nucleotide identity (ANI) to the type genome, which has been assigned the MAG ID ChiSxjej2B14-6234 and which is available via NCBI BioSample SAMN15817054. The GC content of the type genome is $66.20 \%$ and the genome length is 2.4 Mbp.

6952

6953

6954

Description of Candidatus Onthocola gen. nov.

Peer) reviewing PDF | (2020:12:55980:1:1:NEW 13 Jan 2021) 
6955 Candidatus Onthocola (On.tho.co'la. Gr. masc. n. onthos dung; L. suff. -cola inhabitant of; N.L.

6956 fem. n. Onthocola a microbe associated with faeces)

6957

6958

6959

A bacterial genus identified by metagenomic analyses. The genus includes all bacteria with

6960 genomes that show $\geq 60 \%$ average amino acid identity (AAI) to the type genome from the type

6961 species Onthocola gallistercoris. This is a name for the alphanumeric GTDB genus. This genus

6962 has been assigned by GTDB-Tk v1.3.0 working on GTDB Release 05-RS95 (Chaumeil et al.,

6963

6964 2019; Parks et al., 2020) to the order Lachnospirales and to the family Lachnospiraceae.

6966

Description of Candidatus Onthocola gallistercoris sp. nov.

6967 Candidatus Onthocola gallistercoris (gal.li.ster'co.ris. L. masc. n gallus chicken; L. neut. n.

6968 stercus dung; N.L. gen. n. gallistercoris of chicken faeces)

6970

A bacterial species identified by metagenomic analyses. This species includes all bacteria with

6971 genomes that show $\geq 95 \%$ average nucleotide identity (ANI) to the type genome, which has been

6972 assigned the MAG ID CHK187-14744 and which is available via NCBI BioSample

6973 SAMN15817044. The GC content of the type genome is $48.32 \%$ and the genome length is 2.4 Mbp.

6974

6975

6976

Description of Candidatus Onthocola stercoravium sp. nov.

6977 Candidatus Onthocola stercoravium (ster.cor.a'vi.um. L. neut. n. stercus dung; L. fem. n. avis

6978 bird; N.L. gen. n. stercoravium of bird faeces)

6979

6980

A bacterial species identified by metagenomic analyses. This species includes all bacteria with

6981

6982 genomes that show $\geq 95 \%$ average nucleotide identity (ANI) to the type genome, which has been assigned the MAG ID ChiW5-5982 and which is available via NCBI BioSample

6983 SAMN15817021. The GC content of the type genome is $28.08 \%$ and the genome length is 1.4 6984 Mbp.

6985

6986

6987

6988

Description of Candidatus Onthocola stercorigallinarum sp. nov.

Candidatus Onthocola stercorigallinarum (ster.co.ri.gal.li.na'rum. L. neut. n. stercus dung; L.

6989 fem. n. gallina hen; N.L. gen. n. stercorigallinarum of hen faeces)

6990

6991

A bacterial species identified by metagenomic analyses. This species includes all bacteria with

6992

6993 genomes that show $\geq 95 \%$ average nucleotide identity (ANI) to the type genome, which has been

6994 assigned the MAG ID CHK195-3072 and which is available via NCBI BioSample

6995 SAMN15817046. The GC content of the type genome is $27.21 \%$ and the genome length is 1.3

6996

6997

6998 Mbp.

6999

Description of Candidatus Onthomonas gen. nov.

7000

Candidatus Onthomonas (On.tho.mo'nas. Gr. masc. n. onthos dung; L. fem. n. monas a monad; N.L. fem. n. Onthomonas a microbe associated with faeces) 
7001

7002

7003

7004

7005

7006

7007

7008

7009

7010

7011

7012

7013

7014

7015

7016

7017

7018

7019

7020

7021

7022

7023

7024

7025

7026

7027

7028

7029

7030

7031

7032

7033

7034

7035

7036

7037

7038

7039

7040

7041

7042

7043

7044

7045

A bacterial genus identified by metagenomic analyses. The genus includes all bacteria with genomes that show $\geq 60 \%$ average amino acid identity (AAI) to the type genome from the type species Onthomonas avicola. This is a name for the alphanumeric GTDB genus NK3B98. This genus has been assigned by GTDB-Tk v1.3.0 working on GTDB Release 05-RS95 (Chaumeil et al., 2019; Parks et al., 2020) to the order Oscillospirales and to the family Oscillospiraceae.

\section{Description of Candidatus Onthomonas avicola sp. nov.}

Candidatus Onthomonas avicola (a.vi'co.la. L. fem. n. avis bird; L. suff. -cola inhabitant of; N.L. n. avicola inhabitant of birds)

A bacterial species identified by metagenomic analyses. This species includes all bacteria with genomes that show $\geq 95 \%$ average nucleotide identity (ANI) to the type genome, which has been assigned the MAG ID ChiGjej6B6-14002 and which is available via NCBI BioSample SAMN15817096. The GC content of the type genome is $63.01 \%$ and the genome length is 2.4 Mbp.

\section{Description of Candidatus Onthomorpha gen. nov.}

Candidatus Onthomorpha (On.tho.mor'pha. Gr. masc. n. onthos dung; Gr. fem. n. morphe a form, shape; N.L. fem. n. Onthomorpha a microbe associated with faeces)

A bacterial genus identified by metagenomic analyses. The genus includes all bacteria with genomes that show $\geq 60 \%$ average amino acid identity (AAI) to the type genome from the type species Onthomorpha intestinigallinarum. This is a name for the alphanumeric GTDB genus UBA3388. This genus has been assigned by GTDB-Tk v1.3.0 working on GTDB Release 05RS95 (Chaumeil et al., 2019; Parks et al., 2020) to the order Bacteroidales and to the family P3.

\section{Description of Candidatus Onthomorpha intestinigallinarum sp. nov.}

Candidatus Onthomorpha intestinigallinarum (in.tes.ti.ni.gal.li.na'rum. L. neut. n. intestinum gut; L. fem. n. gallina hen; N.L. gen. n. intestinigallinarum of the gut of the hens)

A bacterial species identified by metagenomic analyses. This species includes all bacteria with genomes that show $\geq 95 \%$ average nucleotide identity (ANI) to the type genome, which has been assigned the MAG ID Gambia16-930 and which is available via NCBI BioSample SAMN15817128. The GC content of the type genome is $42.76 \%$ and the genome length is 1.7 Mbp.

\section{Description of Candidatus Onthoplasma gen. nov.}

Candidatus Onthoplasma (On.tho.plas'ma. Gr. masc. n. onthos dung; Gr. neut. n. plasma a form; N.L. neut. n. Onthoplasma a microbe associated with faeces) 
7046 A bacterial genus identified by metagenomic analyses. The genus includes all bacteria with 7047 genomes that show $\geq 60 \%$ average amino acid identity (AAI) to the type genome from the type species Onthoplasma faecipullorum. This is a name for the alphanumeric GTDB genus UBA4626. This genus has been assigned by GTDB-Tk v1.3.0 working on GTDB Release 05RS95 (Chaumeil et al., 2019; Parks et al., 2020) to the order $4 C 28 d-15$ and to the family UBA1242.

7052

7053

7054

7055

Description of Candidatus Onthoplasma faecigallinarum sp. nov.

7056

7057

7058

Candidatus Onthoplasma faecigallinarum (fae.ci.gal.li.na'rum. L. fem. n. faex, faecis excrement; L. fem. n. gallina hen; N.L. gen. n. faecigallinarum of hen faeces)

7059

A bacterial species identified by metagenomic analyses. This species includes all bacteria with genomes that show $\geq 95 \%$ average nucleotide identity (ANI) to the type genome, which has been

7060 assigned the MAG ID 5992 and which is available via NCBI BioSample SAMN15817127. The

7061

7062

7063

7064

7065 $\mathrm{GC}$ content of the type genome is $34.71 \%$ and the genome length is $1.0 \mathrm{Mbp}$.

7066

7067

7068

7069

7070

7071

7072

7073

7074

7075

7076

Description of Candidatus Onthosoma gen. nov.

Description of Candidatus Onthoplasma faecipullorum sp. nov.

Candidatus Onthoplasma faecipullorum (fae.ci.pul.lo'rum. L. fem. n. faex, faecis excrement; L. masc. n. pullus a young chicken; N.L. gen. n. faecipullorum of young chicken faeces)

A bacterial species identified by metagenomic analyses. This species includes all bacteria with genomes that show $\geq 95 \%$ average nucleotide identity (ANI) to the type genome, which has been assigned the MAG ID CHK191-42317 and which is available via NCBI BioSample SAMN15817053. The GC content of the type genome is $31.88 \%$ and the genome length is 1.1 Mbp.

7077

7078

7079

7080

7081

7082

7083

7084

7085

Candidatus Onthosoma (On.tho.so'ma. Gr. masc. n. onthos dung; Gr. neut. n. soma a body; N.L. neut. n. Onthosoma a microbe associated with faeces)

A bacterial genus identified by metagenomic analyses. The genus includes all bacteria with genomes that show $\geq 60 \%$ average amino acid identity (AAI) to the type genome from the type species Onthosoma merdavium. This is a name for the alphanumeric GTDB genus OEMR01. This genus has been assigned by GTDB-Tk v1.3.0 working on GTDB Release 05-RS95 (Chaumeil et al., 2019; Parks et al., 2020) to the order Erysipelotrichales and to the family Erysipelotrichaceae.

7086

7087

7088

Description of Candidatus Onthosoma merdavium sp. nov.

7089

Candidatus Onthosoma merdavium (merd.a'vi.um. L. fem. n. merda faeces; L. fem. n. avis bird; 7090 N.L. gen. n. merdavium of bird faeces) 
7091 A bacterial species identified by metagenomic analyses. This species includes all bacteria with 7092 genomes that show $\geq 95 \%$ average nucleotide identity (ANI) to the type genome, which has been 7093 assigned the MAG ID ChiBcec15-4520 and which is available via NCBI BioSample

7094 SAMN15817169. This is a new name for the alphanumeric GTDB species sp900199515. The $7095 \mathrm{GC}$ content of the type genome is $45.30 \%$ and the genome length is $1.6 \mathrm{Mbp}$.

7096

7097

7098

7099

Description of Candidatus Onthousia gen. nov.

Candidatus Onthousia (Onth.ou'si.a. Gr. masc. n. onthos dung; Gr. fem. n. ousia an essence;

7100 N.L. fem. n. Onthousia a microbe associated with faeces)

7101

7102

A bacterial genus identified by metagenomic analyses. The genus includes all bacteria with

7103

7104

7105 genomes that show $\geq 60 \%$ average amino acid identity (AAI) to the type genome from the type species Onthousia faecavium. This is a name for the alphanumeric GTDB genus CAG-451. This genus has been assigned by GTDB-Tk v1.3.0 working on GTDB Release 05-RS95 (Chaumeil et

7106

7107

7108

7109

7110 al., 2019; Parks et al., 2020) to the order $R F 39$ and to the family $C A G-611$.

7111

7112

7113

7114

7115

7116

\section{Description of Candidatus Onthousia excrementipullorum sp. nov.}

Candidatus Onthousia excrementipullorum (ex.cre.men.ti.pul.lo'rum. L. neut. n. excrementum excrement; L. masc. n. pullus a young chicken; N.L. gen. n. excrementipullorum of young chicken excrement)

A bacterial species identified by metagenomic analyses. This species includes all bacteria with genomes that show $\geq 95 \%$ average nucleotide identity (ANI) to the type genome, which has been assigned the MAG ID CHK184-20233 and which is available via NCBI BioSample

7117

7118 SAMN15817019. The GC content of the type genome is $27.72 \%$ and the genome length is 1.3 Mbp.

7119

7120

7121

7122

7123

7124

7125

7126

7127

7128

7129

\section{Description of Candidatus Onthousia faecavium sp. nov.}

Candidatus Onthousia faecavium (faec.a'vi.um. L. fem. n. faex, faecis excrement; L. fem. n. avis bird; N.L. gen. n. faecavium of bird faeces)

A bacterial species identified by metagenomic analyses. This species includes all bacteria with genomes that show $\geq 95 \%$ average nucleotide identity (ANI) to the type genome, which has been assigned the MAG ID CHK195-6217 and which is available via NCBI BioSample SAMN15817026. The GC content of the type genome is $28.40 \%$ and the genome length is 1.3 Mbp.

7130

7131

7132

7133

7134

7135

Description of Candidatus Onthousia faecigallinarum sp. nov. Candidatus Onthousia faecigallinarum (fae.ci.gal.li.na'rum. L. fem. n. faex, faecis excrement; L. fem. n. gallina hen; N.L. gen. n. faecigallinarum of hen faeces) 
7136 A bacterial species identified by metagenomic analyses. This species includes all bacteria with 7137 genomes that show $\geq 95 \%$ average nucleotide identity (ANI) to the type genome, which has been

7138 assigned the MAG ID CHK135-1819 and which is available via NCBI BioSample

7139 SAMN15817105. The GC content of the type genome is $32.62 \%$ and the genome length is 1.1

7140 Mbp.

7141

7142

7143

7144

7145

7146

7147

7148

7149

7150

7151

7152

7153

7154

7155

7156

7157

7158

7159

7160

7161

7162

7163

7164

7165

7166

7167

Description of Candidatus Onthousia faecipullorum sp. nov.

Candidatus Onthousia faecipullorum (fae.ci.pul.lo'rum. L. fem. n. faex, faecis excrement; L. masc. n. pullus a young chicken; N.L. gen. n. faecipullorum of young chicken faeces)

A bacterial species identified by metagenomic analyses. This species includes all bacteria with genomes that show $\geq 95 \%$ average nucleotide identity (ANI) to the type genome, which has been assigned the MAG ID CHK195-26880 and which is available via NCBI BioSample SAMN15817040. The GC content of the type genome is $27.41 \%$ and the genome length is 1.4 Mbp.

\section{Description of Candidatus Onthovicinus gen. nov.}

Candidatus Onthovicinus (On.tho.vic.in'us. Gr. masc. n. onthos dung; L. masc. n. vicinus a neighbour; N.L. masc. n. Onthovicinus a microbe associated with faeces)

A bacterial genus identified by metagenomic analyses. The genus includes all bacteria with genomes that show $\geq 60 \%$ average amino acid identity (AAI) to the type genome from the type species Onthovicinus excrementipullorum. This is a name for the alphanumeric GTDB genus UMGS1839. This genus has been assigned by GTDB-Tk v1.3.0 working on GTDB Release 05RS95 (Chaumeil et al., 2019; Parks et al., 2020) to the order Oscillospirales and to the family Acutalibacteraceae.

\section{Description of Candidatus Onthovicinus excrementipullorum sp. nov.}

Candidatus Onthovicinus excrementipullorum (ex.cre.men.ti.pul.lo'rum. L. neut. n. excrementum

7168

7169

7170

7171

7172

7173

7174

7175 excrement; L. masc. n. pullus a young chicken; N.L. gen. n. excrementipullorum of young chicken excrement)

7176

7177

7178

7179

A bacterial species identified by metagenomic analyses. This species includes all bacteria with genomes that show $\geq 95 \%$ average nucleotide identity (ANI) to the type genome, which has been assigned the MAG ID CHK185-12131 and which is available via NCBI BioSample SAMN15817020. The GC content of the type genome is $55.55 \%$ and the genome length is 2.4 Mbp.

7180

Description of Candidatus Onthovivens gen. nov. Candidatus Onthovivens (On.tho.viv'ens. Gr. masc. n. onthos dung; N.L. pres. part. vivens living; N.L. fem. n. Onthovivens a microbe associated with faeces)

7181 
7182 A bacterial genus identified by metagenomic analyses. The genus includes all bacteria with 7183 genomes that show $\geq 60 \%$ average amino acid identity (AAI) to the type genome from the type

7184 species Onthovivens merdipullorum. This is a name for the alphanumeric GTDB genus

7185 UBA4855. This genus has been assigned by GTDB-Tk v1.3.0 working on GTDB Release 05-

7186 RS95 (Chaumeil et al., 2019; Parks et al., 2020) to the order RFN20 and to the family CAG-826.

7187

7188

7189

7190

7191

7192

7193

7194

7195

7196

7197

7198

7199

7200

7201

7202

7203

7204

7205

7206

7207

7208

7209

7210

7211

7212

7213

7214

7215

7216

7217

7218

7219

7220

7221

7222

7223

7224

7225

7226

7227

\section{Description of Candidatus Onthovivens merdipullorum sp. nov.}

Candidatus Onthovivens merdipullorum (mer.di.pul.lo'rum. L. fem. n. merda faeces; L. masc. $n$. pullus a young chicken; N.L. gen. n. merdipullorum of the faeces of young chickens)

A bacterial species identified by metagenomic analyses. This species includes all bacteria with genomes that show $\geq 95 \%$ average nucleotide identity (ANI) to the type genome, which has been assigned the MAG ID 11159 and which is available via NCBI BioSample SAMN15817139. The $\mathrm{GC}$ content of the type genome is $27.05 \%$ and the genome length is $1.5 \mathrm{Mbp}$.

\section{Description of Candidatus Ornithocaccomicrobium gen. nov.}

Candidatus Ornithocaccomicrobium (Or.ni.tho.cac.co.mi.cro'bi.um. Gr. masc. or fem. n. ornis, ornithos bird; Gr. fem. n. kakke faeces; N.L. neut. n. microbium a microbe; N.L. neut. n.

Ornithocaccomicrobium A microbe found in chicken faceces)

A bacterial genus identified by metagenomic analyses. The genus includes all bacteria with genomes that show $\geq 60 \%$ average amino acid identity (AAI) to the type genome from the type species Ornithocaccomicrobium faecavium. This genus was identified but not named by Glendinning et al. This genus has been assigned by GTDB-Tk v1.3.0 working on GTDB Release 05-RS95 (Chaumeil et al., 2019; Parks et al., 2020) to the order Christensenellales and to the family $C A G-74$.

\section{Description of Candidatus Ornithocaccomicrobium faecavium sp. nov.}

Candidatus Ornithocaccomicrobium faecavium (faec.a'vi.um. L. fem. n. faex, faecis excrement; L. fem. n. avis bird; N.L. gen. n. faecavium of bird faeces)

A bacterial species identified by metagenomic analyses. This species includes all bacteria with genomes that show $\geq 95 \%$ average nucleotide identity (ANI) to the type genome, which has been assigned the MAG ID CHK183-6373 and which is available via NCBI BioSample SAMN15816945. The GC content of the type genome is $59.48 \%$ and the genome length is 2.9 Mbp.

\section{Description of Candidatus Ornithoclostridium gen. nov.}

Candidatus Ornithoclostridium (Or.ni.tho.clos.tri'di.um. Gr. masc. or fem. n. ornis, ornithos bird; N.L. neut. n. Clostridium a genus name; N.L. neut. n. Ornithoclostridium a genus related to the genus Clostridium but distinct from it and found in poultry) 
7228

7229

7230

7231

7232

7233

7234

7235

7236

7237

7238

7239

7240

7241

7242

7243

7244

7245

7246

7247

7248

7249

7250

7251

7252

7253

7254

7255

7256

7257

7258

7259

7260

7261

7262

7263

7264

7265

7266

7267

7268

7269

7270

7271

7272

A bacterial genus identified by metagenomic analyses. The genus includes all bacteria with genomes that show $\geq 60 \%$ average amino acid identity (AAI) to the type genome from the type species Ornithoclostridium excrementipullorum. This genus has been assigned by GTDB-Tk v1.3.0 working on GTDB Release 05-RS95 (Chaumeil et al., 2019; Parks et al., 2020) to the order Christensenellales and to the family UBA3700.

\section{Description of Candidatus Ornithoclostridium excrementipullorum sp. nov.} Candidatus Ornithoclostridium excrementipullorum (ex.cre.men.ti.pul.lo'rum. L. neut. n. excrementum excrement; L. masc. n. pullus a young chicken; N.L. gen. n. excrementipullorum of young chicken excrement)

A bacterial species identified by metagenomic analyses. This species includes all bacteria with genomes that show $\geq 95 \%$ average nucleotide identity (ANI) to the type genome, which has been assigned the MAG ID ChiW5-1639 and which is available via NCBI BioSample SAMN15816971. The GC content of the type genome is $54.62 \%$ and the genome length is 1.6 Mbp.

\section{Description of Candidatus Ornithoclostridium faecavium sp. nov.}

Candidatus Ornithoclostridium faecavium (faec.a'vi.um. L. fem. n. faex, faecis excrement; L. fem. n. avis bird; N.L. gen. n. faecavium of bird faeces)

A bacterial species identified by metagenomic analyses. This species includes all bacteria with genomes that show $\geq 95 \%$ average nucleotide identity (ANI) to the type genome, which has been assigned the MAG ID 63 and which is available via NCBI BioSample SAMN15816992. The GC content of the type genome is $48.09 \%$ and the genome length is $1.8 \mathrm{Mbp}$.

\section{Description of Candidatus Ornithoclostridium faecigallinarum sp. nov.} Candidatus Ornithoclostridium faecigallinarum (fae.ci.gal.li.na'rum. L. fem. n. faex, faecis excrement; L. fem. n. gallina hen; N.L. gen. n. faecigallinarum of hen faeces)

A bacterial species identified by metagenomic analyses. This species includes all bacteria with genomes that show $\geq 95 \%$ average nucleotide identity (ANI) to the type genome, which has been assigned the MAG ID ChiHcolR4-3946 and which is available via NCBI BioSample SAMN15816996. The GC content of the type genome is $57.12 \%$ and the genome length is 1.6 Mbp.

\section{Description of Candidatus Ornithomonoglobus gen. nov.} Candidatus Ornithomonoglobus (Or.ni.tho.mo.no.glo'bus. Gr. masc. or fem. n. ornis, ornithos bird; N.L. masc. n. Monoglobus a genus name; N.L. masc. n. Ornithomonoglobus a genus related to the genus Monoglobus but distinct from it and found in poultry) 
7273 A bacterial genus identified by metagenomic analyses. The genus includes all bacteria with

7274

7275

7276

7277

7278

7279

7280

7281

7282

7283

7284

7285

7286

7287

7288

7289

7290

7291

7292

7293

7294

7295

7296

7297

7298

7299

7300

7301

7302

7303

7304

7305

7306

7307

7308

7309

7310

7311

7312

7313

7314

7315

7316

7317

7318

genomes that show $\geq 60 \%$ average amino acid identity (AAI) to the type genome from the type species Ornithomonoglobus merdipullorum. This genus was identified but not named by Glendinning et al. This genus has been assigned by GTDB-Tk v1.3.0 working on GTDB Release 05-RS95 (Chaumeil et al., 2019; Parks et al., 2020) to the order Monoglobales and to the family UBA1381.

\section{Description of Candidatus Ornithomonoglobus intestinigallinarum sp. nov.}

Candidatus Ornithomonoglobus intestinigallinarum (in.tes.ti.ni.gal.li.na'rum. L. neut. n. intestinum gut; L. fem. n. gallina hen; N.L. gen. n. intestinigallinarum of the gut of the hens)

A bacterial species identified by metagenomic analyses. This species includes all bacteria with genomes that show $\geq 95 \%$ average nucleotide identity (ANI) to the type genome, which has been assigned the MAG ID CHK181-108 and which is available via NCBI BioSample SAMN15816941. The GC content of the type genome is $49.36 \%$ and the genome length is 2.2 Mbp.

\section{Description of Candidatus Ornithomonoglobus merdipullorum sp. nov.} Candidatus Ornithomonoglobus merdipullorum (mer.di.pul.lo'rum. L. fem. n. merda faeces; L. masc. n. pullus a young chicken; N.L. gen. n. merdipullorum of the faeces of young chickens)

A bacterial species identified by metagenomic analyses. This species includes all bacteria with genomes that show $\geq 95 \%$ average nucleotide identity (ANI) to the type genome, which has been assigned the MAG ID USAMLcec3-3695 and which is available via NCBI BioSample SAMN15816942. The GC content of the type genome is $48.52 \%$ and the genome length is 2.7 Mbp.

\section{Description of Candidatus Ornithospirochaeta gen. nov.}

Candidatus Ornithospirochaeta (Or.ni.tho.spi.ro.chae'ta. Gr. masc. or fem. n. ornis, ornithos bird; N.L. fem. n. Spirochaeta a genus name; N.L. fem. n. Ornithospirochaeta a genus related to the genus Spirochaeta but distinct from it and found in poultry)

A bacterial genus identified by metagenomic analyses. The genus includes all bacteria with genomes that show $\geq 60 \%$ average amino acid identity (AAI) to the type genome from the type species Ornithospirochaeta stercoravium. This genus has been assigned by GTDB-Tk v1.3.0 working on GTDB Release 05-RS95 (Chaumeil et al., 2019; Parks et al., 2020) to the order Sphaerochaetales and to the family Sphaerochaetaceae.

\section{Description of Candidatus Ornithospirochaeta avicola sp. nov.}

Candidatus Ornithospirochaeta avicola (a.vi'co.la. L. fem. n. avis bird; L. suff. -cola inhabitant of; N.L. n. avicola inhabitant of birds)

Peer) reviewing PDF | (2020:12:55980:1:1:NEW 13 Jan 2021) 
7319 A bacterial species identified by metagenomic analyses. This species includes all bacteria with 7320 genomes that show $\geq 95 \%$ average nucleotide identity (ANI) to the type genome, which has been

7321

7322

7323

7324

7325

7326

7327

7328

7329

7330

7331

7332

7333

7334

7335

7336

7337

7338

7339

7340

7341

7342

7343

7344

7345

7346

7347

7348

7349

7350

7351

7352

7353

7354

7355

7356

7357

7358

7359

7360

7361

7362

7363

7364 assigned the MAG ID Gambia11-129 and which is available via NCBI BioSample SAMN15816993. The GC content of the type genome is $42.81 \%$ and the genome length is 1.5 Mbp.

\section{Description of Candidatus Ornithospirochaeta stercoravium sp. nov.} Candidatus Ornithospirochaeta stercoravium (ster.cor.a'vi.um. L. neut. n. stercus dung; L. fem. n. avis bird; N.L. gen. n. stercoravium of bird faeces)

A bacterial species identified by metagenomic analyses. This species includes all bacteria with genomes that show $\geq 95 \%$ average nucleotide identity (ANI) to the type genome, which has been assigned the MAG ID 14700 and which is available via NCBI BioSample SAMN15816953. The GC content of the type genome is $46.26 \%$ and the genome length is $2.0 \mathrm{Mbp}$.

\section{Description of Candidatus Ornithospirochaeta stercorigallinarum sp. nov.}

Candidatus Ornithospirochaeta stercorigallinarum (ster.co.ri.gal.li.na'rum. L. neut. n. stercus dung; L. fem. n. gallina hen; N.L. gen. n. stercorigallinarum of hen faeces)

A bacterial species identified by metagenomic analyses. This species includes all bacteria with genomes that show $\geq 95 \%$ average nucleotide identity (ANI) to the type genome, which has been assigned the MAG ID ChiHecec3B27-9561 and which is available via NCBI BioSample SAMN15816957. The GC content of the type genome is $46.77 \%$ and the genome length is 1.9 Mbp.

\section{Description of Candidatus Ornithospirochaeta stercoripullorum sp. nov.}

Candidatus Ornithospirochaeta stercoripullorum (ster.co.ri.pul.lo'rum. L. neut. n. stercus dung; L. masc. n. pullus a young chicken; N.L. gen. n. stercoripullorum of the faceces of young chickens)

A bacterial species identified by metagenomic analyses. This species includes all bacteria with genomes that show $\geq 95 \%$ average nucleotide identity (ANI) to the type genome, which has been assigned the MAG ID 7293 and which is available via NCBI BioSample SAMN15816978. The $\mathrm{GC}$ content of the type genome is $45.57 \%$ and the genome length is $2.0 \mathrm{Mbp}$.

\section{Description of Candidatus Oscillibacter avistercoris sp. nov.}

Candidatus Oscillibacter avistercoris (a.vi.ster'co.ris. L. fem. n. avis bird; L. neut. n. stercus dung; N.L. gen. n. avistercoris of bird faeces)

A bacterial species identified by metagenomic analyses. This species includes all bacteria with genomes that show $\geq 95 \%$ average nucleotide identity (ANI) to the type genome, which has been assigned the MAG ID CHK176-14096 and which is available via NCBI BioSample

Peer) reviewing PDF | (2020:12:55980:1:1:NEW 13 Jan 2021) 
7365 SAMN15816820. This is a new name for the alphanumeric GTDB species sp900556925. The

7366

7367

7368

7369

7370

7371

7372

7373

7374

7375

7376

7377

7378

7379

7380

7381

7382

7383

7384

7385

7386

7387

7388

7389

7390

7391

7392

7393

7394

7395

7396

7397

7398

7399

7400

7401

7402

7403

7404

7405

7406

7407

7408

7409

7410

$\mathrm{GC}$ content of the type genome is $63.55 \%$ and the genome length is $2.3 \mathrm{Mbp}$.

\author{
Description of Candidatus Oscillibacter excrementavium sp. nov. \\ Candidatus Oscillibacter excrementavium (ex.cre.ment.a'vi.um. L. neut. n. excrementum \\ excrement; L. fem. n. avis bird; N.L. gen. n. excrementavium of bird excrement)
}

A bacterial species identified by metagenomic analyses. This species includes all bacteria with genomes that show $\geq 95 \%$ average nucleotide identity (ANI) to the type genome, which has been assigned the MAG ID 5302 and which is available via NCBI BioSample SAMN15816661. The $\mathrm{GC}$ content of the type genome is $63.73 \%$ and the genome length is $2.5 \mathrm{Mbp}$.

\title{
Description of Candidatus Oscillibacter excrementigallinarum sp. nov. Candidatus Oscillibacter excrementigallinarum (ex.cre.men.ti.gal.li.na'rum. L. neut. $\mathrm{n}$. excrementum excrement; L. fem. n. gallina hen; N.L. gen. n. excrementigallinarum of hen excrement)
}

A bacterial species identified by metagenomic analyses. This species includes all bacteria with genomes that show $\geq 95 \%$ average nucleotide identity (ANI) to the type genome, which has been assigned the MAG ID ChiBcec18-1249 and which is available via NCBI BioSample SAMN15816667. The GC content of the type genome is $64.01 \%$ and the genome length is 2.4 Mbp.

\section{Description of Candidatus Oscillibacter pullicola sp. nov.}

Candidatus Oscillibacter pullicola (pul.li'co.la. L. masc. n. pullus a young chicken; L. suff. -cola inhabitant of; N.L. n. pullicola an inhabitant of young chickens)

A bacterial species identified by metagenomic analyses. This species includes all bacteria with genomes that show $\geq 95 \%$ average nucleotide identity (ANI) to the type genome, which has been assigned the MAG ID ChiBcolR2-4535 and which is available via NCBI BioSample SAMN15816652. The GC content of the type genome is $63.62 \%$ and the genome length is 2.4 Mbp.

\section{Description of Candidatus Paenalcaligenes intestinipullorum sp. nov.}

Candidatus Paenalcaligenes intestinipullorum (in.tes.ti.ni.pul.lo'rum. L. neut. n. intestinum gut; L. masc. n. pullus a young chicken; N.L. gen. n. intestinipullorum of the gut of young chickens)

A bacterial species identified by metagenomic analyses. This species includes all bacteria with genomes that show $\geq 95 \%$ average nucleotide identity (ANI) to the type genome, which has been assigned the MAG ID 9264 and which is available via NCBI BioSample SAMN15816786. The $\mathrm{GC}$ content of the type genome is $51.92 \%$ and the genome length is $1.8 \mathrm{Mbp}$. 
7411

7412

7413

7414

7415

7416

7417

7418

7419

7420

7421

7422

7423

7424

7425

7426

7427

7428

7429

7430

7431

7432

7433

7434

7435

7436

7437

7438

7439

7440

7441

7442

7443

7444

7445

7446

7447

7448

7449

7450

7451

7452

7453

7454

7455

7456

Description of Candidatus Paenibacillus intestinavium sp. nov.

Candidatus Paenibacillus intestinavium (in.tes.tin.a'vi.um. L. neut. n. intestinum gut; L. fem. n. avis bird; N.L. gen. n. intestinavium of the gut of birds)

A bacterial species identified by metagenomic analyses. This species includes all bacteria with genomes that show $\geq 95 \%$ average nucleotide identity (ANI) to the type genome, which has been assigned the MAG ID CHK172-12487 and which is available via NCBI BioSample SAMN15816909. Although GTDB has assigned this species to the genus it calls Paenibacillus_C , this genus designation cannot be incorporated into a well-formed binomial, so in naming this species, we have used the current validly published name for the genus. The GC content of the type genome is $39.31 \%$ and the genome length is $4.6 \mathrm{Mbp}$.

\section{Description of Candidatus Parabacteroides faecavium sp. nov.}

Candidatus Parabacteroides faecavium (faec.a'vi.um. L. fem. n. faex, faecis excrement; L. fem. n. avis bird; N.L. gen. n. faecavium of bird faeces)

A bacterial species identified by metagenomic analyses. This species includes all bacteria with genomes that show $\geq 95 \%$ average nucleotide identity (ANI) to the type genome, which has been assigned the MAG ID CHK152-2511 and which is available via NCBI BioSample SAMN15816864. This is a new name for the alphanumeric GTDB species sp000436495. The $\mathrm{GC}$ content of the type genome is $42.40 \%$ and the genome length is $3.4 \mathrm{Mbp}$.

\section{Description of Candidatus Parabacteroides intestinavium sp. nov.}

Candidatus Parabacteroides intestinavium (in.tes.tin.a'vi.um. L. neut. n. intestinum gut; L. fem. n. avis bird; N.L. gen. n. intestinavium of the gut of birds)

A bacterial species identified by metagenomic analyses. This species includes all bacteria with genomes that show $\geq 95 \%$ average nucleotide identity (ANI) to the type genome, which has been assigned the MAG ID ChiHjej11B10-3189 and which is available via NCBI BioSample SAMN15816658. The GC content of the type genome is $44.93 \%$ and the genome length is 2.8 Mbp.

\section{Description of Candidatus Parabacteroides intestinigallinarum sp. nov.}

Candidatus Parabacteroides intestinigallinarum (in.tes.ti.ni.gal.li.na'rum. L. neut. n. intestinum gut; L. fem. n. gallina hen; N.L. gen. n. intestinigallinarum of the gut of the hens)

A bacterial species identified by metagenomic analyses. This species includes all bacteria with genomes that show $\geq 95 \%$ average nucleotide identity (ANI) to the type genome, which has been assigned the MAG ID ChiHecec2B26-12326 and which is available via NCBI BioSample SAMN15816728. The GC content of the type genome is $52.90 \%$ and the genome length is 2.9 Mbp. 
Description of Candidatus Parabacteroides intestinipullorum sp. nov.

7459

Candidatus Parabacteroides intestinipullorum (in.tes.ti.ni.pul.lo'rum. L. neut. n. intestinum gut;

7460

L. masc. n. pullus a young chicken; N.L. gen. n. intestinipullorum of the gut of young chickens)

7461

7462

A bacterial species identified by metagenomic analyses. This species includes all bacteria with

7463

7464 genomes that show $\geq 95 \%$ average nucleotide identity (ANI) to the type genome, which has been assigned the MAG ID ChiGjej6B6-14162 and which is available via NCBI BioSample

7465 SAMN15816857. This is a new name for the alphanumeric GTDB species sp900552415. The $\mathrm{GC}$ content of the type genome is $50.53 \%$ and the genome length is $3.2 \mathrm{Mbp}$.

\section{Description of Candidatus Paralactobacillus gallistercoris sp. nov.}

7470 Candidatus Paralactobacillus gallistercoris (gal.li.ster'co.ris. L. masc. n gallus chicken; L. neut. n. stercus dung; N.L. gen. n. gallistercoris of chicken faeces)

A bacterial species identified by metagenomic analyses. This species includes all bacteria with genomes that show $\geq 95 \%$ average nucleotide identity (ANI) to the type genome, which has been assigned the MAG ID F6-6636 and which is available via NCBI BioSample SAMN15816781. The GC content of the type genome is $35.69 \%$ and the genome length is $1.2 \mathrm{Mbp}$.

\section{Description of Candidatus Paraprevotella stercoravium sp. nov.}

7480 Candidatus Paraprevotella stercoravium (ster.cor.a'vi.um. L. neut. n. stercus dung; L. fem. n. avis bird; N.L. gen. n. stercoravium of bird faeces)

A bacterial species identified by metagenomic analyses. This species includes all bacteria with genomes that show $\geq 95 \%$ average nucleotide identity (ANI) to the type genome, which has been assigned the MAG ID G3-2149 and which is available via NCBI BioSample SAMN15816669. The $\mathrm{GC}$ content of the type genome is $45.06 \%$ and the genome length is $3.2 \mathrm{Mbp}$.

\section{Description of Candidatus Paraprevotella stercorigallinarum sp. nov.}

Candidatus Paraprevotella stercorigallinarum (ster.co.ri.gal.li.na'rum. L. neut. n. stercus dung; L. fem. n. gallina hen; N.L. gen. n. stercorigallinarum of hen faeces)

A bacterial species identified by metagenomic analyses. This species includes all bacteria with

\section{Description of Candidatus Parasutterella gallistercoris sp. nov.} Candidatus Parasutterella gallistercoris (gal.li.ster'co.ris. L. masc. n gallus chicken; L. neut. n. stercus dung; N.L. gen. n. gallistercoris of chicken faeces) 
7503

7504

7505

7506

7507

7508

7509

7510

7511

7512

7513

7514

7515

7516

7517

7518

7519

7520

7521

7522

7523

7524

7525

7526

7527

7528

7529

7530

7531

7532

7533

7534

7535

7536

7537

7538

7539

7540

7541

7542

7543

7544

7545

7546

7547

A bacterial species identified by metagenomic analyses. This species includes all bacteria with genomes that show $\geq 95 \%$ average nucleotide identity (ANI) to the type genome, which has been assigned the MAG ID 21611 and which is available via NCBI BioSample SAMN15816870. This is a new name for the alphanumeric GTDB species sp000980495. The GC content of the type genome is $49.58 \%$ and the genome length is $1.9 \mathrm{Mbp}$.

\section{Description of Candidatus Pelethenecus gen. nov.}

Candidatus Pelethenecus (Pel.eth.en.e'cus. Gr. masc. n. pelethos dung; Gr. masc. enoikos inhabitant; N.L. masc. n. Pelethenecus a microbe associated with faeces)

A bacterial genus identified by metagenomic analyses. The genus includes all bacteria with genomes that show $\geq 60 \%$ average amino acid identity (AAI) to the type genome from the type species Pelethenecus faecipullorum. This is a name for the alphanumeric GTDB genus UMGS268. This genus has been assigned by GTDB-Tk v1.3.0 working on GTDB Release 05RS95 (Chaumeil et al., 2019; Parks et al., 2020) to the order Acholeplasmatales and to the family Anaeroplasmataceae.

\section{Description of Candidatus Pelethenecus faecipullorum sp. nov.}

Candidatus Pelethenecus faecipullorum (ster.co.ri.gal.li.na'rum. L. fem. n. faex, faecis excrement; L. masc. n. pullus a young chicken; N.L. gen. n. faecipullorum of young chicken faeces)

A bacterial species identified by metagenomic analyses. This species includes all bacteria with genomes that show $\geq 95 \%$ average nucleotide identity (ANI) to the type genome, which has been assigned the MAG ID ChiW17-6978 and which is available via NCBI BioSample SAMN15817226. This is a new name for the alphanumeric GTDB species sp900540175. The $\mathrm{GC}$ content of the type genome is $39.85 \%$ and the genome length is $1.3 \mathrm{Mbp}$.

\section{Description of Candidatus Pelethocola gen. nov.}

Candidatus Pelethocola (Pel.eth.o.co'la. Gr. masc. n. pelethos dung; L. suff. -cola inhabitant of; N.L. fem. n. Pelethocola a microbe associated with faeces)

A bacterial genus identified by metagenomic analyses. The genus includes all bacteria with genomes that show $\geq 60 \%$ average amino acid identity (AAI) to the type genome from the type species Pelethocola excrementipullorum. This is a name for the alphanumeric GTDB genus UBA5416. This genus has been assigned by GTDB-Tk v1.3.0 working on GTDB Release 05RS95 (Chaumeil et al., 2019; Parks et al., 2020) to the order Lachnospirales and to the family Lachnospiraceae.

\section{Description of Candidatus Pelethocola excrementipullorum sp. nov.}


7548 Candidatus Pelethocola excrementipullorum (ex.cre.men.ti.pul.lo'rum. L. neut. n. excrementum

7549

7550

7551

7552

7553

7554

7555

7556

7557

7558

7559

7560

7561

7562

7563

7564

7565

7566

7567

7568

7569

7570

7571

7572

7573

7574

7575

7576

7577

7578

7579

7580

7581

7582

7583

7584

7585

7586

7587

7588

7589

7590

7591

7592

7593

excrement; L. masc. n. pullus a young chicken; N.L. gen. n. excrementipullorum of young

chicken excrement)

A bacterial species identified by metagenomic analyses. This species includes all bacteria with genomes that show $\geq 95 \%$ average nucleotide identity (ANI) to the type genome, which has been assigned the MAG ID CHK160-5124 and which is available via NCBI BioSample

SAMN15817143. The GC content of the type genome is $43.72 \%$ and the genome length is 3.9 Mbp.

\section{Description of Candidatus Pelethomonas gen. nov.}

Candidatus Pelethomonas (Pel.eth.omo'nas. Gr. masc. n. pelethos dung; L. fem. n. monas a monad; N.L. fem. n. Pelethomonas a microbe associated with faeces)

A bacterial genus identified by metagenomic analyses. The genus includes all bacteria with genomes that show $\geq 60 \%$ average amino acid identity (AAI) to the type genome from the type species Pelethomonas intestinigallinarum. This is a name for the alphanumeric GTDB genus UMGS1872. This genus has been assigned by GTDB-Tk v1.3.0 working on GTDB Release 05RS95 (Chaumeil et al., 2019; Parks et al., 2020) to the order Oscillospirales and to the family Oscillospiraceae.

\section{Description of Candidatus Pelethomonas intestinigallinarum sp. nov.}

Candidatus Pelethomonas intestinigallinarum (in.tes.ti.ni.gal.li.na'rum. L. neut. n. intestinum gut; L. fem. n. gallina hen; N.L. gen. n. intestinigallinarum of the gut of the hens)

A bacterial species identified by metagenomic analyses. This species includes all bacteria with genomes that show $\geq 95 \%$ average nucleotide identity (ANI) to the type genome, which has been assigned the MAG ID ChiSjej2B20-3600 and which is available via NCBI BioSample SAMN15817014. The GC content of the type genome is $63.89 \%$ and the genome length is 2.2 Mbp.

\section{Description of Candidatus Pelethosoma gen. nov.}

Candidatus Pelethosoma (Pel.eth.o.so'ma. Gr. masc. n. pelethos dung; Gr. neut. n. soma a body; N.L. neut. n. Pelethosoma a microbe associated with faeces)

A bacterial genus identified by metagenomic analyses. The genus includes all bacteria with genomes that show $\geq 60 \%$ average amino acid identity (AAI) to the type genome from the type species Pelethosoma merdigallinarum. This is a name for the alphanumeric GTDB genus UMGS2016. This genus has been assigned by GTDB-Tk v1.3.0 working on GTDB Release 05RS95 (Chaumeil et al., 2019; Parks et al., 2020) to the order RF39 and to the family CAG-822.

\section{Description of Candidatus Pelethosoma merdigallinarum sp. nov.}


7594 Candidatus Pelethosoma merdigallinarum (mer.di.gal.li.na'rum. L. fem. n. merda faeces; L. fem.

7595

7596

7597

7598

7599

7600

7601

7602

7603

7604

7605

7606

7607

7608

7609

7610

7611

7612

7613

7614

7615

7616

7617

7618

7619

7620

7621

7622

7623

7624

7625

7626

7627

7628

7629

7630

7631

7632

7633

7634

7635

7636

7637

7638

n. gallina hen; N.L. gen. n. merdigallinarum of hen faeces)

A bacterial species identified by metagenomic analyses. This species includes all bacteria with genomes that show $\geq 95 \%$ average nucleotide identity (ANI) to the type genome, which has been assigned the MAG ID CHK195-5794 and which is available via NCBI BioSample SAMN15817039. The GC content of the type genome is $30.57 \%$ and the genome length is 1.3 Mbp.

\section{Description of Candidatus Pelethousia gen. nov.}

Candidatus Pelethousia (Pel.eth.ou'si.a. Gr. masc. n. pelethos dung; Gr. fem. n. ousia an essence; N.L. fem. n. Pelethousia a microbe associated with faeces)

A bacterial genus identified by metagenomic analyses. The genus includes all bacteria with genomes that show $\geq 60 \%$ average amino acid identity (AAI) to the type genome from the type species Pelethousia gallinarum. This is a name for the alphanumeric GTDB genus UBA5394. This genus has been assigned by GTDB-Tk v1.3.0 working on GTDB Release 05-RS95 (Chaumeil et al., 2019; Parks et al., 2020) to the order Christensenellales and to the family $C A G$ 138 .

\section{Description of Candidatus Pelethousia gallinarum sp. nov.}

Candidatus Pelethousia gallinarum (gal.li.na'rum. L. fem. n. gallina a hen; L. fem. gen.pl. n. gallinarum of hens)

A bacterial species identified by metagenomic analyses. This species includes all bacteria with genomes that show $\geq 95 \%$ average nucleotide identity (ANI) to the type genome, which has been assigned the MAG ID ChiHcec27-1353 and which is available via NCBI BioSample SAMN15817178. This is a new name for the alphanumeric GTDB species sp003150565. The $\mathrm{GC}$ content of the type genome is $59.40 \%$ and the genome length is $2.0 \mathrm{Mbp}$.

\section{Description of Candidatus Phascolarctobacterium stercoravium sp. nov.}

Candidatus Phascolarctobacterium stercoravium (ster.cor.a'vi.um. L. neut. n. stercus dung; L. fem. n. avis bird; N.L. gen. n. stercoravium of bird faeces)

A bacterial species identified by metagenomic analyses. This species includes all bacteria with genomes that show $\geq 95 \%$ average nucleotide identity (ANI) to the type genome, which has been assigned the MAG ID ChiBcec14-732 and which is available via NCBI BioSample SAMN15816834. This is a new name for the alphanumeric GTDB species sp000436095. The GC content of the type genome is $46.50 \%$ and the genome length is $1.7 \mathrm{Mbp}$.

\section{Description of Candidatus Phocaeicola caecigallinarum sp. nov.}


7639 Candidatus Phocaeicola caecigallinarum (cae.ci.gal.li.na'rum. L. neut. n. caecum the caecum; L.

7640

7641

7642

7643

7644

7645

7646

7647

7648

7649

7650

7651

7652

7653

7654

7655

7656

7657

7658

7659

7660

7661

7662

7663

7664

7665

7666

7667

7668

7669

7670

7671

7672

7673

7674

7675

7676

7677

7678

7679

7680

7681

7682

7683

7684

fem. n. gallina a hen; N.L. gen. n. caecigallinarum of the caecum of hens)

A bacterial species identified by metagenomic analyses. This species includes all bacteria with genomes that show $\geq 95 \%$ average nucleotide identity (ANI) to the type genome, which has been assigned the MAG ID ChiHjej11B10-3694 and which is available via NCBI BioSample SAMN15816802. This is a new name for the alphanumeric GTDB species sp002161565. The GC content of the type genome is $46.18 \%$ and the genome length is $3.2 \mathrm{Mbp}$.

\section{Description of Candidatus Phocaeicola excrementigallinarum sp. nov. \\ Candidatus Phocaeicola excrementigallinarum (ex.cre.men.ti.gal.li.na'rum. L. neut. n. excrementum excrement; L. fem. n. gallina hen; N.L. gen. n. excrementigallinarum of hen excrement)}

A bacterial species identified by metagenomic analyses. This species includes all bacteria with genomes that show $\geq 95 \%$ average nucleotide identity (ANI) to the type genome, which has been assigned the MAG ID 12279 and which is available via NCBI BioSample SAMN15816632. The $\mathrm{GC}$ content of the type genome is $50.57 \%$ and the genome length is $2.5 \mathrm{Mbp}$.

\section{Description of Candidatus Phocaeicola excrementipullorum sp. nov.}

Candidatus Phocaeicola excrementipullorum (ex.cre.men.ti.pul.lo'rum. L. neut. n. excrementum excrement; L. masc. n. pullus a young chicken; N.L. gen. n. excrementipullorum of young chicken excrement)

A bacterial species identified by metagenomic analyses. This species includes all bacteria with genomes that show $\geq 95 \%$ average nucleotide identity (ANI) to the type genome, which has been assigned the MAG ID 8470 and which is available via NCBI BioSample SAMN15816808. This is a new name for the alphanumeric GTDB species sp900546095. The GC content of the type genome is $49.09 \%$ and the genome length is $3.1 \mathrm{Mbp}$.

\section{Description of Candidatus Phocaeicola faecigallinarum sp. nov. Candidatus Phocaeicola faecigallinarum (fae.ci.gal.li.na'rum. L. fem. n. faex, faecis excrement; L. fem. n. gallina hen; N.L. gen. n. faecigallinarum of hen faeces)}

A bacterial species identified by metagenomic analyses. This species includes all bacteria with genomes that show $\geq 95 \%$ average nucleotide identity (ANI) to the type genome, which has been assigned the MAG ID G4-2901 and which is available via NCBI BioSample SAMN15816657. The GC content of the type genome is $40.33 \%$ and the genome length is $3.3 \mathrm{Mbp}$.

\section{Description of Candidatus Phocaeicola faecipullorum sp. nov.} Candidatus Phocaeicola faecipullorum (fae.ci.pul.lo'rum. L. fem. n. faex, faecis excrement; L. masc. n. pullus a young chicken; N.L. gen. n. faecipullorum of young chicken faeces) 
7685

7686

7687

7688

7689

7690

7691

7692

7693

7694

7695

7696

7697

7698

7699

7700

7701

7702

7703

7704

7705

7706

7707

7708

7709

7710

7711

7712

7713

7714

7715

7716

7717

7718

7719

7720

7721

7722

7723

7724

7725

7726

7727

7728

7729

7730

A bacterial species identified by metagenomic analyses. This species includes all bacteria with genomes that show $\geq 95 \%$ average nucleotide identity (ANI) to the type genome, which has been assigned the MAG ID 17637 and which is available via NCBI BioSample SAMN15816682. The $\mathrm{GC}$ content of the type genome is $39.94 \%$ and the genome length is $3.9 \mathrm{Mbp}$.

\section{Description of Candidatus Phocaeicola gallinarum sp. nov.}

Candidatus Phocaeicola gallinarum (gal.li.na'rum. L. fem. n. gallina a hen; L. fem. n. avis bird; N.L. gen. n. faecavium of bird faeces)

A bacterial species identified by metagenomic analyses. This species includes all bacteria with genomes that show $\geq 95 \%$ average nucleotide identity (ANI) to the type genome, which has been assigned the MAG ID ChiGjej6B6-595 and which is available via NCBI BioSample SAMN15816805. This is a new name for the alphanumeric GTDB species sp900540105. The GC content of the type genome is $45.79 \%$ and the genome length is $2.8 \mathrm{Mbp}$.

\section{Description of Candidatus Phocaeicola gallistercoris sp. nov.}

Candidatus Phocaeicola gallistercoris (gal.li.ster'co.ris. L. masc. n gallus chicken; L. neut. n. stercus dung; N.L. gen. n. gallistercoris of chicken faeces)

A bacterial species identified by metagenomic analyses. This species includes all bacteria with genomes that show $\geq 95 \%$ average nucleotide identity (ANI) to the type genome, which has been assigned the MAG ID Gambia9-593 and which is available via NCBI BioSample SAMN15816698. The GC content of the type genome is $38.34 \%$ and the genome length is 2.3 Mbp.

\section{Description of Candidatus Phocaeicola merdavium sp. nov.}

Candidatus Phocaeicola merdavium (merd.a'vi.um. L. fem. n. merda faeces; L. fem. n. avis bird; N.L. gen. n. merdavium of bird faeces)

A bacterial species identified by metagenomic analyses. This species includes all bacteria with genomes that show $\geq 95 \%$ average nucleotide identity (ANI) to the type genome, which has been assigned the MAG ID CHK136-5299 and which is available via NCBI BioSample SAMN15816804. This is a new name for the alphanumeric GTDB species sp002161765. The $\mathrm{GC}$ content of the type genome is $44.54 \%$ and the genome length is $2.6 \mathrm{Mbp}$.

\section{Description of Candidatus Phocaeicola merdigallinarum sp. nov.}

Candidatus Phocaeicola merdigallinarum (mer.di.gal.li.na'rum. L. fem. n. merda faeces; L. fem. n. gallina hen; N.L. gen. n. merdigallinarum of hen faeces)

A bacterial species identified by metagenomic analyses. This species includes all bacteria with genomes that show $\geq 95 \%$ average nucleotide identity (ANI) to the type genome, which has been

Peer) reviewing PDF | (2020:12:55980:1:1:NEW 13 Jan 2021) 
7731

7732

7733

7734

7735

7736

7737

7738

7739

7740

7741

7742

7743

7744

7745

7746

7747

7748

7749

7750

7751

7752

7753

7754

7755

7756

7757

7758

7759

7760

7761

7762

7763

7764

7765

7766

7767

7768

7769

7770

7771

7772

7773

7774

7775

7776

assigned the MAG ID 17689 and which is available via NCBI BioSample SAMN15816829. This is a new name for the alphanumeric GTDB species sp900066455. The GC content of the type genome is $46.19 \%$ and the genome length is $3.3 \mathrm{Mbp}$.

\section{Description of Candidatus Prevotella avicola sp. nov.}

Candidatus Prevotella avicola (a.vi'co.la. L. fem. n. avis bird; L. suff. -cola inhabitant of; N.L. n. avicola inhabitant of birds)

A bacterial species identified by metagenomic analyses. This species includes all bacteria with genomes that show $\geq 95 \%$ average nucleotide identity (ANI) to the type genome, which has been assigned the MAG ID ChiHecec3B27-8219 and which is available via NCBI BioSample SAMN15816846. This is a new name for the alphanumeric GTDB species sp000435635. The GC content of the type genome is $51.22 \%$ and the genome length is $1.9 \mathrm{Mbp}$.

\section{Description of Candidatus Prevotella intestinigallinarum sp. nov.}

Candidatus Prevotella intestinigallinarum (in.tes.ti.ni.gal.li.na'rum. L. neut. n. intestinum gut; L. fem. n. gallina hen; N.L. gen. n. intestinigallinarum of the gut of the hens)

A bacterial species identified by metagenomic analyses. This species includes all bacteria with genomes that show $\geq 95 \%$ average nucleotide identity (ANI) to the type genome, which has been assigned the MAG ID 146 and which is available via NCBI BioSample SAMN15816872. This is a new name for the alphanumeric GTDB species sp900540415. The GC content of the type genome is $56.37 \%$ and the genome length is $2.9 \mathrm{Mbp}$.

\section{Description of Candidatus Prevotella stercoripullorum sp. nov.}

Candidatus Prevotella stercoripullorum (ster.co.ri.pul.lo'rum. L. neut. n. stercus dung; L. masc. n. pullus a young chicken; N.L. gen. n. stercoripullorum of the faceces of young chickens)

A bacterial species identified by metagenomic analyses. This species includes all bacteria with genomes that show $\geq 95 \%$ average nucleotide identity (ANI) to the type genome, which has been assigned the MAG ID USASDec6-549 and which is available via NCBI BioSample SAMN15816866. This is a new name for the alphanumeric GTDB species sp900554045. The GC content of the type genome is $53.35 \%$ and the genome length is $2.5 \mathrm{Mbp}$.

\section{Description of Candidatus Protoclostridium stercorigallinarum sp. nov.}

Candidatus Protoclostridium stercorigallinarum (ster.co.ri.gal.li.na'rum. L. neut. n. stercus dung; L. fem. n. gallina hen; N.L. gen. n. stercorigallinarum of hen faeces)

A bacterial species identified by metagenomic analyses. This species includes all bacteria with genomes that show $\geq 95 \%$ average nucleotide identity (ANI) to the type genome, which has been assigned the MAG ID 12435 and which is available via NCBI BioSample SAMN15816772. The GC content of the type genome is $56.70 \%$ and the genome length is $1.7 \mathrm{Mbp}$. 
7777

7778

7779

7780

7781

7782

7783

7784

7785

7786

7787

7788

7789

7790

7791

7792

7793

7794

7795

7796

7797

7798

7799

7800

7801

7802

7803

7804

7805

7806

7807

7808

7809

7810

7811

7812

7813

7814

7815

7816

7817

7818

7819

7820

7821

7822
Description of Candidatus Pseudogracilibacillus intestinigallinarum sp. nov. Candidatus Pseudogracilibacillus intestinigallinarum (in.tes.ti.ni.gal.li.na'rum. L. neut. n. intestinum gut; L. fem. n. gallina hen; N.L. gen. n. intestinigallinarum of the gut of the hens)

A bacterial species identified by metagenomic analyses. This species includes all bacteria with genomes that show $\geq 95 \%$ average nucleotide identity (ANI) to the type genome, which has been assigned the MAG ID CHK169-2315 and which is available via NCBI BioSample SAMN15816775. The GC content of the type genome is $35.03 \%$ and the genome length is 2.5 Mbp.

\section{Description of Candidatus Pseudomonas excrementavium sp. nov.}

Candidatus Pseudomonas excrementavium (ex.cre.ment.a'vi.um. L. neut. n. excrementum excrement; L. fem. n. avis bird; N.L. gen. n. excrementavium of bird excrement)

A bacterial species identified by metagenomic analyses. This species includes all bacteria with genomes that show $\geq 95 \%$ average nucleotide identity (ANI) to the type genome, which has been assigned the MAG ID CHK174-787 and which is available via NCBI BioSample SAMN15816898. Although GTDB has assigned this species to the genus it calls Pseudomonas_D, this genus designation cannot be incorporated into a well-formed binomial, so in naming this species, we have used the current validly published name for the genus. The GC content of the type genome is $61.84 \%$ and the genome length is $3.0 \mathrm{Mbp}$.

\section{Description of Candidatus Pullibacteroides gen. nov.}

Candidatus Pullibacteroides (Pul.li.bac.te.ro'i.des. L. masc. n. pullus a young chicken; N.L. masc. n. Bacteroides a genus name; N.L. masc. n. Pullibacteroides a genus related to the genus Bacteroides but distinct from it and found in poultry)

A bacterial genus identified by metagenomic analyses. The genus includes all bacteria with genomes that show $\geq 60 \%$ average amino acid identity (AAI) to the type genome from the type species Pullibacteroides excrementavium. This genus has been assigned by GTDB-Tk v1.3.0 working on GTDB Release 05-RS95 (Chaumeil et al., 2019; Parks et al., 2020) to the order Bacteroidales and to the family $P 3$.

\section{Description of Candidatus Pullibacteroides excrementavium sp. nov.}

Candidatus Pullibacteroides excrementavium (ex.cre.ment.a'vi.um. L. neut. n. excrementum excrement; L. fem. n. avis bird; N.L. gen. n. excrementavium of bird excrement)

A bacterial species identified by metagenomic analyses. This species includes all bacteria with genomes that show $\geq 95 \%$ average nucleotide identity (ANI) to the type genome, which has been assigned the MAG ID 2889 and which is available via NCBI BioSample SAMN15816989. The $\mathrm{GC}$ content of the type genome is $51.34 \%$ and the genome length is $2.4 \mathrm{Mbp}$.

Peer] reviewing PDF | (2020:12:55980:1:1:NEW 13 Jan 2021) 
7823

7824

7825

7826

7827

7828

7829

7830

7831

7832

7833

7834

7835

7836

7837

7838

7839

7840

7841

7842

7843

7844

7845

7846

7847

7848

7849

7850

7851

7852

7853

7854

7855

7856

7857

7858

7859

7860

7861

7862

7863

7864

7865

7866

7867

7868

Description of Candidatus Pullichristensenella gen. nov.

Candidatus Pullichristensenella (Pul.li.chris.ten.sen.el'la. L. masc. n. pullus a young chicken; N.L. fem. n. Christensenella a genus name; N.L. fem. n. Pullichristensenella a genus related to the genus Christensenella but distinct from it and found in poultry)

A bacterial genus identified by metagenomic analyses. The genus includes all bacteria with genomes that show $\geq 60 \%$ average amino acid identity (AAI) to the type genome from the type species Pullichristensenella avicola. This genus has been assigned by GTDB-Tk v1.3.0 working on GTDB Release 05-RS95 (Chaumeil et al., 2019; Parks et al., 2020) to the order

Christensenellales and to the family $C A G-74$.

\section{Description of Candidatus Pullichristensenella avicola sp. nov.}

Candidatus Pullichristensenella avicola (a.vi'co.la. L. fem. n. avis bird; L. suff. -cola inhabitant of; N.L. n. avicola inhabitant of birds)

A bacterial species identified by metagenomic analyses. This species includes all bacteria with genomes that show $\geq 95 \%$ average nucleotide identity (ANI) to the type genome, which has been assigned the MAG ID 10205 and which is available via NCBI BioSample SAMN15816956. The $\mathrm{GC}$ content of the type genome is $63.02 \%$ and the genome length is $2.3 \mathrm{Mbp}$.

\section{Description of Candidatus Pullichristensenella excrementigallinarum sp. nov.}

Candidatus Pullichristensenella excrementigallinarum (ex.cre.men.ti.gal.li.na'rum. L. neut. n. excrementum excrement; L. fem. n. gallina hen; N.L. gen. n. excrementigallinarum of hen excrement)

A bacterial species identified by metagenomic analyses. This species includes all bacteria with genomes that show $\geq 95 \%$ average nucleotide identity (ANI) to the type genome, which has been assigned the MAG ID ChiHcec3-11533 and which is available via NCBI BioSample SAMN15816983. The GC content of the type genome is $57.19 \%$ and the genome length is 2.2 Mbp.

\section{Description of Candidatus Pullichristensenella excrementipullorum sp. nov.}

Candidatus Pullichristensenella excrementipullorum (ex.cre.men.ti.pul.lo'rum. L. neut. n. excrementum excrement; L. masc. n. pullus a young chicken; N.L. gen. n. excrementipullorum of young chicken excrement)

A bacterial species identified by metagenomic analyses. This species includes all bacteria with genomes that show $\geq 95 \%$ average nucleotide identity (ANI) to the type genome, which has been assigned the MAG ID 1279 and which is available via NCBI BioSample SAMN15817001. The $\mathrm{GC}$ content of the type genome is $63.33 \%$ and the genome length is $2.7 \mathrm{Mbp}$.

Peer) reviewing PDF | (2020:12:55980:1:1:NEW 13 Jan 2021) 
7869

7870

7871

7872

7873

7874

7875

7876

7877

7878

7879

7880

7881

7882

7883

7884

7885

7886

7887

7888

7889

7890

7891

7892

7893

7894

7895

7896

7897

7898

7899

7900

7901

7902

7903

7904

7905

7906

7907

7908

7909

7910

7911

7912

7913

7914

\section{Description of Candidatus Pullichristensenella stercorigallinarum sp. nov.}

Candidatus Pullichristensenella stercorigallinarum (ster.co.ri.gal.li.na'rum. L. neut. n. stercus dung; L. fem. n. gallina hen; N.L. gen. n. stercorigallinarum of hen faeces)

A bacterial species identified by metagenomic analyses. This species includes all bacteria with genomes that show $\geq 95 \%$ average nucleotide identity (ANI) to the type genome, which has been assigned the MAG ID ChiSjej6B24-2974 and which is available via NCBI BioSample SAMN15816933. The GC content of the type genome is $60.06 \%$ and the genome length is 2.7 Mbp.

\section{Description of Candidatus Pullichristensenella stercoripullorum sp. nov.}

Candidatus Pullichristensenella stercoripullorum (ster.co.ri.pul.lo'rum. L. neut. n. stercus dung; L. masc. n. pullus a young chicken; N.L. gen. n. stercoripullorum of the faceces of young chickens)

A bacterial species identified by metagenomic analyses. This species includes all bacteria with genomes that show $\geq 95 \%$ average nucleotide identity (ANI) to the type genome, which has been assigned the MAG ID 5266 and which is available via NCBI BioSample SAMN15816952. The $\mathrm{GC}$ content of the type genome is $64.02 \%$ and the genome length is $2.3 \mathrm{Mbp}$.

\section{Description of Candidatus Pullilachnospira gen. nov.}

Candidatus Pullilachnospira (Pul.li.lach.no.spi'ra. L. masc. n. pullus a young chicken; N.L. fem. n. Lachnospira a genus name; N.L. fem. n. Pullilachnospira a genus related to the genus Lachnospira but distinct from it and found in poultry)

A bacterial genus identified by metagenomic analyses. The genus includes all bacteria with genomes that show $\geq 60 \%$ average amino acid identity (AAI) to the type genome from the type species Pullilachnospira stercoravium. This genus was identified but not named by Glendinning et al. This genus has been assigned by GTDB-Tk v1.3.0 working on GTDB Release 05-RS95 (Chaumeil et al., 2019; Parks et al., 2020) to the order Lachnospirales and to the family Lachnospiraceae.

\section{Description of Candidatus Pullilachnospira gallistercoris sp. nov.}

Candidatus Pullilachnospira gallistercoris (gal.li.ster'co.ris. L. masc. n gallus chicken; L. neut. n. stercus dung; N.L. gen. n. gallistercoris of chicken faeces)

A bacterial species identified by metagenomic analyses. This species includes all bacteria with genomes that show $\geq 95 \%$ average nucleotide identity (ANI) to the type genome, which has been assigned the MAG ID ChiSjej5B23-6657 and which is available via NCBI BioSample SAMN15816936. The GC content of the type genome is $53.36 \%$ and the genome length is 2.5 Mbp. 
7915

7916

7917

7918

7919

7920

7921

7922

7923

7924

7925

7926

7927

7928

7929

7930

7931

7932

7933

7934

7935

7936

7937

7938

7939

7940

7941

7942

7943

7944

7945

7946

7947

7948

7949

7950

7951

7952

7953

7954

7955

7956

7957

7958

7959

7960

Description of Candidatus Pullilachnospira intestinigallinarum sp. nov.

Candidatus Pullilachnospira intestinigallinarum (in.tes.ti.ni.gal.li.na'rum. L. neut. n. intestinum gut; L. fem. n. gallina hen; N.L. gen. n. intestinigallinarum of the gut of the hens)

A bacterial species identified by metagenomic analyses. This species includes all bacteria with genomes that show $\geq 95 \%$ average nucleotide identity (ANI) to the type genome, which has been assigned the MAG ID CHK192-16996 and which is available via NCBI BioSample SAMN15816938. The GC content of the type genome is $51.71 \%$ and the genome length is 2.8 Mbp.

\section{Description of Candidatus Pullilachnospira stercoravium sp. nov.}

Candidatus Pullilachnospira stercoravium (ster.cor.a'vi.um. L. neut. n. stercus dung; L. fem. n. avis bird; N.L. gen. n. stercoravium of bird faeces)

A bacterial species identified by metagenomic analyses. This species includes all bacteria with genomes that show $\geq 95 \%$ average nucleotide identity (ANI) to the type genome, which has been assigned the MAG ID ChiBcec2-4451 and which is available via NCBI BioSample SAMN15816944. The GC content of the type genome is $53.14 \%$ and the genome length is 2.8 Mbp.

\section{Description of Candidatus Pygmaiobacter gallistercoris sp. nov.}

Candidatus Pygmaiobacter gallistercoris (gal.li.ster'co.ris. L. masc. n. gallus chicken; L. neut. n. stercus dung; N.L. gen. n. gallistercoris of chicken faeces)

A bacterial species identified by metagenomic analyses. This species includes all bacteria with genomes that show $\geq 95 \%$ average nucleotide identity (ANI) to the type genome, which has been assigned the MAG ID ChiGjej6B6-17065 and which is available via NCBI BioSample

SAMN15816776. The GC content of the type genome is $61.74 \%$ and the genome length is 1.6 Mbp.

\section{Description of Candidatus Rikenella faecigallinarum sp. nov.}

Candidatus Rikenella faecigallinarum (fae.ci.gal.li.na'rum. L. fem. n. faex, faecis excrement; L. fem. n. gallina hen; N.L. gen. n. faecigallinarum of hen faeces)

A bacterial species identified by metagenomic analyses. This species includes all bacteria with genomes that show $\geq 95 \%$ average nucleotide identity (ANI) to the type genome, which has been assigned the MAG ID ChiBcec15-1070 and which is available via NCBI BioSample SAMN15816768. The GC content of the type genome is $56.15 \%$ and the genome length is 1.8 Mbp.

Description of Candidatus Rothia avicola sp. nov.

Peer] reviewing PDF | (2020:12:55980:1:1:NEW 13 Jan 2021) 
7961 Candidatus Rothia avicola (a.vi'co.la. L. fem. n. avis bird; L. suff. -cola inhabitant of; N.L. n. 7962 avicola inhabitant of birds)

7963

7964

7965

7966

7967

7968

7969

7970

7971

7972

7973

7974

7975

7976

7977

7978

7979

7980

7981

7982

7983

7984

7985

7986

7987

7988

7989

7990

7991

7992

7993

7994

7995

7996

7997

7998

7999

8000

8001

8002

8003

8004

8005

8006

A bacterial species identified by metagenomic analyses. This species includes all bacteria with genomes that show $\geq 95 \%$ average nucleotide identity (ANI) to the type genome, which has been assigned the MAG ID ChiHjej12B11-9195 and which is available via NCBI BioSample SAMN15816701. The GC content of the type genome is $60.05 \%$ and the genome length is 2.1 Mbp.

\section{Description of Candidatus Rothia avistercoris sp. nov.}

Candidatus Rothia avistercoris (a.vi.ster'co.ris. L. fem. n. avis bird; L. neut. n. stercus dung; N.L. gen. n. avistercoris of bird faeces)

A bacterial species identified by metagenomic analyses. This species includes all bacteria with genomes that show $\geq 95 \%$ average nucleotide identity (ANI) to the type genome, which has been assigned the MAG ID ChiHjej10B9-4811 and which is available via NCBI BioSample SAMN15816788. The GC content of the type genome is $59.79 \%$ and the genome length is 2.0 Mbp.

\section{Description of Candidatus Ruania gallistercoris sp. nov.}

Candidatus Ruania gallistercoris (gal.li.ster'co.ris. L. masc. n. gallus chicken; L. neut. n. stercus dung; N.L. gen. n. gallistercoris of chicken faeces)

A bacterial species identified by metagenomic analyses. This species includes all bacteria with genomes that show $\geq 95 \%$ average nucleotide identity (ANI) to the type genome, which has been assigned the MAG ID ChiGjej4B4-7305 and which is available via NCBI BioSample

SAMN15816695. The GC content of the type genome is $69.62 \%$ and the genome length is 4.4 Mbp.

\section{Description of Candidatus Rubneribacter avistercoris sp. nov.}

Candidatus Rubneribacter avistercoris (a.vi.ster'co.ris. L. fem. n. avis bird; L. neut. n. stercus dung; N.L. gen. n. avistercoris of bird faeces)

A bacterial species identified by metagenomic analyses. This species includes all bacteria with genomes that show $\geq 95 \%$ average nucleotide identity (ANI) to the type genome, which has been assigned the MAG ID ChiGjej6B6-20359 and which is available via NCBI BioSample SAMN15816703. The GC content of the type genome is $65.17 \%$ and the genome length is 3.2 Mbp.

\section{Description of Candidatus Ruminococcus avistercoris sp. nov.} Candidatus Ruminococcus avistercoris (a.vi.ster'co.ris. L. fem. n. avis bird; L. neut. n. stercus dung; N.L. gen. n. avistercoris of bird faeces) 
8007

8008

8009

8010

8011

8012

8013

8014

8015

8016

8017

8018

8019

8020

8021

8022

8023

8024

8025

8026

8027

8028

8029

8030

8031

8032

8033

8034

8035

8036

8037

8038

8039

8040

8041

8042

8043

8044

8045

8046

8047

8048

8049

8050

8051

8052

A bacterial species identified by metagenomic analyses. This species includes all bacteria with genomes that show $\geq 95 \%$ average nucleotide identity (ANI) to the type genome, which has been assigned the MAG ID CHK186-6582 and which is available via NCBI BioSample SAMN15816883. Although GTDB has assigned this species to the genus it calls Ruminococcus_G, this genus designation cannot be incorporated into a well-formed binomial, so in naming this species, we have used the current validly published name for the genus. The $\mathrm{GC}$ content of the type genome is $50.07 \%$ and the genome length is $2.3 \mathrm{Mbp}$.

\section{Description of Candidatus Ruminococcus gallistercoris sp. nov.}

Candidatus Ruminococcus gallistercoris (gal.li.ster'co.ris. L. masc. n gallus chicken; L. neut. n. stercus dung; N.L. gen. n. gallistercoris of chicken faeces)

A bacterial species identified by metagenomic analyses. This species includes all bacteria with genomes that show $\geq 95 \%$ average nucleotide identity (ANI) to the type genome, which has been assigned the MAG ID ChiBcec12-341 and which is available via NCBI BioSample SAMN15816918. This is a new name for the alphanumeric GTDB species sp900552925. Although GTDB has assigned this species to the genus it calls Ruminococcus_H, this genus designation cannot be incorporated into a well-formed binomial, so in naming this species, we have used the current validly published name for the genus. The GC content of the type genome is $62.03 \%$ and the genome length is $2.1 \mathrm{Mbp}$.

\section{Description of Candidatus Ruminococcus intestinipullorum sp. nov.}

Candidatus Ruminococcus intestinipullorum (in.tes.ti.ni.pul.lo'rum. L. neut. n. intestinum gut; L. masc. n. pullus a young chicken; N.L. gen. n. intestinipullorum of the gut of young chickens)

A bacterial species identified by metagenomic analyses. This species includes all bacteria with genomes that show $\geq 95 \%$ average nucleotide identity (ANI) to the type genome, which has been assigned the MAG ID 1485 and which is available via NCBI BioSample SAMN15816905. Although GTDB has assigned this species to the genus it calls Ruminococcus_B, this genus designation cannot be incorporated into a well-formed binomial, so in naming this species, we have used the current validly published name for the genus. The GC content of the type genome is $35.77 \%$ and the genome length is $2.1 \mathrm{Mbp}$.

\section{Description of Candidatus Ruthenibacterium avium sp. nov. \\ Candidatus Ruthenibacterium avium (a'vi.um. L. fem. pl. n. avium of birds)}

A bacterial species identified by metagenomic analyses. This species includes all bacteria with genomes that show $\geq 95 \%$ average nucleotide identity (ANI) to the type genome, which has been assigned the MAG ID ChiBcec8-14828 and which is available via NCBI BioSample SAMN15816823. This is a new name for the alphanumeric GTDB species sp002315015. The GC content of the type genome is $51.27 \%$ and the genome length is $2.2 \mathrm{Mbp}$. 
8053

8054

8055

8056

8057

8058

8059

8060

8061

8062

8063

8064

8065

8066

8067

8068

8069

8070

8071

8072

8073

8074

8075

8076

8077

8078

8079

8080

8081

8082

8083

8084

8085

8086

8087

8088

8089

8090

8091

8092

8093

8094

8095

8096

8097

Description of Candidatus Ruthenibacterium merdavium sp. nov.

Candidatus Ruthenibacterium merdavium (merd.a'vi.um. L. fem. n. merda faeces; L. fem. n. avis bird; N.L. gen. n. merdavium of bird faeces)

A bacterial species identified by metagenomic analyses. This species includes all bacteria with genomes that show $\geq 95 \%$ average nucleotide identity (ANI) to the type genome, which has been assigned the MAG ID 5933 and which is available via NCBI BioSample SAMN15816578. The $\mathrm{GC}$ content of the type genome is $51.15 \%$ and the genome length is $2.0 \mathrm{Mbp}$.

\section{Description of Candidatus Ruthenibacterium merdigallinarum sp. nov.}

Candidatus Ruthenibacterium merdigallinarum (mer.di.gal.li.na'rum. L. fem. n. merda faeces; L. fem. n. gallina hen; N.L. gen. n. merdigallinarum of hen faeces)

A bacterial species identified by metagenomic analyses. This species includes all bacteria with genomes that show $\geq 95 \%$ average nucleotide identity (ANI) to the type genome, which has been assigned the MAG ID ChiSjej6B24-7098 and which is available via NCBI BioSample SAMN15816763. The GC content of the type genome is $65.28 \%$ and the genome length is 2.2 Mbp.

\section{Description of Candidatus Ruthenibacterium merdipullorum sp. nov.} Candidatus Ruthenibacterium merdipullorum (mer.di.pul.lo'rum. L. fem. n. merda faeces; L. masc. n. pullus a young chicken; N.L. gen. n. merdipullorum of the faeces of young chickens)

A bacterial species identified by metagenomic analyses. This species includes all bacteria with genomes that show $\geq 95 \%$ average nucleotide identity (ANI) to the type genome, which has been assigned the MAG ID ChiSxjej5B17-15602 and which is available via NCBI BioSample SAMN15816878. This is a new name for the alphanumeric GTDB species sp900546885. The $\mathrm{GC}$ content of the type genome is $59.69 \%$ and the genome length is $2.1 \mathrm{Mbp}$.

\section{Description of Candidatus Salinicoccus merdavium sp. nov.}

Candidatus Salinicoccus merdavium (merd.a'vi.um. L. fem. n. merda faeces; L. fem. n. avis bird; N.L. gen. n. merdavium of bird faeces)

A bacterial species identified by metagenomic analyses. This species includes all bacteria with genomes that show $\geq 95 \%$ average nucleotide identity (ANI) to the type genome, which has been assigned the MAG ID ChiHjej12B11-20095 and which is available via NCBI BioSample SAMN15816874. This is a new name for the alphanumeric GTDB species sp002360325. The GC content of the type genome is $44.20 \%$ and the genome length is $1.8 \mathrm{Mbp}$.

\section{Description of Candidatus Salinicoccus stercoripullorum sp. nov.}


8098 Candidatus Salinicoccus stercoripullorum (ster.co.ri.pul.lo'rum. L. neut. n. stercus dung; L.

8099 masc. n. pullus a young chicken; N.L. gen. n. stercoripullorum of the faceces of young chickens)

8100

8101

8102

8103

A bacterial species identified by metagenomic analyses. This species includes all bacteria with genomes that show $\geq 95 \%$ average nucleotide identity (ANI) to the type genome, which has been assigned the MAG ID ChiHjej13B12-752 and which is available via NCBI BioSample

8104 SAMN15816771. The GC content of the type genome is $48.14 \%$ and the genome length is 2.3

8105 Mbp.

8106

8107

Description of Candidatus Savagella gallinarum sp. nov.

8109 Candidatus Savagella gallinarum (gal.li.na'rum. L. fem. n. gallina a hen; L. fem. n. gallina hen;

8110

8111

8112 L. gen.pl. n. gallinarum of hens)

8113

A bacterial species identified by metagenomic analyses. This species includes all bacteria with

8114 genomes that show $\geq 95 \%$ average nucleotide identity (ANI) to the type genome, which has been

8115 assigned the MAG ID CHK166-5537 and which is available via NCBI BioSample

8116 SAMN15816803. This is a new name for the alphanumeric GTDB species sp001655775. The

8117 $\mathrm{GC}$ content of the type genome is $26.42 \%$ and the genome length is $1.8 \mathrm{Mbp}$.

8118

8119

\section{Description of Candidatus Scatarchaeum gen. nov.}

8120

8121 Candidatus Scatarchaeum (Scat.archaeum. Gr. neut. n. skor, skatos dung; N.L. neut. n. archaeum an archaeon; N.L. neut. n. Scatarchaeum a microbe associated with faeces)

8122

8123

A bacterial genus identified by metagenomic analyses. The genus includes all bacteria with

8124

8125 genomes that show $\geq 60 \%$ average amino acid identity (AAI) to the type genome from the type species Scatarchaeum merdigallinarum. This is a name for the alphanumeric GTDB genus UMGS403. This genus has been assigned by GTDB-Tk v1.3.0 working on GTDB Release 05RS95 (Chaumeil et al., 2019; Parks et al., 2020) to the order Oscillospirales and to the family

8128 Acutalibacteraceae.

8129

8130

\section{Description of Candidatus Scatarchaeum merdigallinarum sp. nov.}

8132 Candidatus Scatarchaeum merdigallinarum (mer.di.gal.li.na'rum. L. fem. n. merda faeces; L.

8133

8134

8135 fem. n. gallina hen; N.L. gen. n. merdigallinarum of hen faeces)

8136

8137

A bacterial species identified by metagenomic analyses. This species includes all bacteria with genomes that show $\geq 95 \%$ average nucleotide identity (ANI) to the type genome, which has been

8138 assigned the MAG ID ChiSjej1B19-3389 and which is available via NCBI BioSample

8139 SAMN15817212. This is a new name for the alphanumeric GTDB species sp900541975. The

8140 $\mathrm{GC}$ content of the type genome is $46.67 \%$ and the genome length is $1.7 \mathrm{Mbp}$.

\section{Description of Candidatus Scatenecus gen. nov.}


8143 Candidatus Scatenecus (Scat.en.e'cus. Gr. neut. n. skor, skatos dung; Gr. masc. enoikos

8144 inhabitant; N.L. masc. n. Scatenecus a microbe associated with the intestines)

8145

8146

8147

8148

8149

8150

8151

A bacterial genus identified by metagenomic analyses. The genus includes all bacteria with genomes that show $\geq 60 \%$ average amino acid identity (AAI) to the type genome from the type species Scatenecus faecavium. This is a name for the alphanumeric GTDB genus QAMI01. This genus has been assigned by GTDB-Tk v1.3.0 working on GTDB Release 05-RS95 (Chaumeil et al., 2019; Parks et al., 2020) to the order Gastranaerophilales and to the family Gastranaerophilaceae.

8152

8153

8154

\section{Description of Candidatus Scatenecus faecavium sp. nov.}

8155

8156

8157

8158 Candidatus Scatenecus faecavium (faec.a'vi.um. L. fem. n. faex, faecis excrement; L. fem. n. avis bird; N.L. gen. n. faecavium of bird faeces)

8159

A bacterial species identified by metagenomic analyses. This species includes all bacteria with

8160 genomes that show $\geq 95 \%$ average nucleotide identity (ANI) to the type genome, which has been

8161 assigned the MAG ID CHK152-2994 and which is available via NCBI BioSample

8162 SAMN15817221. This is a new name for the alphanumeric GTDB species sp900551915. The

8163

8164

8165

8166 $\mathrm{GC}$ content of the type genome is $37.15 \%$ and the genome length is $1.9 \mathrm{Mbp}$.

8168

8169

8170

8171

\section{Description of Candidatus Scatocola gen. nov.}

Candidatus Scatocola (Scat.o.co'la. Gr. neut. n. skor, skatos dung; L. suff. -cola inhabitant of; N.L. fem. n. Scatocola a microbe associated with faeces)

A bacterial genus identified by metagenomic analyses. The genus includes all bacteria with genomes that show $\geq 60 \%$ average amino acid identity (AAI) to the type genome from the type species Scatocola faecipullorum. This is a name for the alphanumeric GTDB genus CAG-495.

8172

8173

8174

8175

8176 This genus has been assigned by GTDB-Tk v1.3.0 working on GTDB Release 05-RS95 (Chaumeil et al., 2019; Parks et al., 2020) to the order RF32 and to the family CAG-239.

8177

\section{Description of Candidatus Scatocola faecigallinarum sp. nov.} Candidatus Scatocola faecigallinarum (fae.ci.gal.li.na'rum. L. fem. n. faex, faecis excrement; L.

8178

8179

8180 fem. n. gallina hen; N.L. gen. n. faecigallinarum of hen faeces)

8181

8182

A bacterial species identified by metagenomic analyses. This species includes all bacteria with genomes that show $\geq 95 \%$ average nucleotide identity (ANI) to the type genome, which has been

8183 assigned the MAG ID 2846 and which is available via NCBI BioSample SAMN15817209. This

8184

8185 is a new name for the alphanumeric GTDB species sp000436375. The GC content of the type genome is $49.31 \%$ and the genome length is $1.7 \mathrm{Mbp}$.

\section{Description of Candidatus Scatocola faecipullorum sp. nov.}


8188 Candidatus Scatocola faecipullorum (fae.ci.pul.lo'rum. L. fem. n. faex, faecis excrement; L. 8189 masc. n. pullus a young chicken; N.L. gen. n. faecipullorum of young chicken faeces)

8190

8191

8192

8193

8194

8195

8196

8197

8198

8199

8200

8201

8202

8203

8204

8205

8206

8207

8208

8209

8210

8211

8212

8213

8214

8215

8216

8217

8218

8219

8220

8221

8222

8223

8224

8225

8226

8227

8228

8229

8230

8231

8232

A bacterial species identified by metagenomic analyses. This species includes all bacteria with genomes that show $\geq 95 \%$ average nucleotide identity (ANI) to the type genome, which has been assigned the MAG ID ChiW3-316 and which is available via NCBI BioSample SAMN15817201. This is a new name for the alphanumeric GTDB species sp001917125. The GC content of the type genome is $47.24 \%$ and the genome length is $1.7 \mathrm{Mbp}$.

\section{Description of Candidatus Scatomonas gen. nov.}

Candidatus Scatomonas (Scat.o.mo'nas. Gr. neut. n. skor, skatos dung; L. fem. n. monas a monad; N.L. fem. n. Scatomonas a microbe associated with the intestines)

A bacterial genus identified by metagenomic analyses. The genus includes all bacteria with genomes that show $\geq 60 \%$ average amino acid identity (AAI) to the type genome from the type species Scatomonas merdigallinarum. This is a name for the alphanumeric GTDB genus OF0933XD. This genus has been assigned by GTDB-Tk v1.3.0 working on GTDB Release 05-RS95 (Chaumeil et al., 2019; Parks et al., 2020) to the order Lachnospirales and to the family Lachnospiraceae.

\section{Description of Candidatus Scatomonas merdavium sp. nov.}

Candidatus Scatomonas merdavium (merd.a'vi.um. L. fem. n. merda faeces; L. fem. n. avis bird; N.L. gen. n. merdavium of bird faeces)

A bacterial species identified by metagenomic analyses. This species includes all bacteria with genomes that show $\geq 95 \%$ average nucleotide identity (ANI) to the type genome, which has been assigned the MAG ID ChiSjej5B23-9500 and which is available via NCBI BioSample SAMN15817033. The GC content of the type genome is $53.14 \%$ and the genome length is 2.3 Mbp.

\section{Description of Candidatus Scatomonas merdigallinarum sp. nov.} Candidatus Scatomonas merdigallinarum (mer.di.gal.li.na'rum. L. fem. n. merda faeces; L. fem. n. gallina hen; N.L. gen. n. merdigallinarum of hen faeces)

A bacterial species identified by metagenomic analyses. This species includes all bacteria with genomes that show $\geq 95 \%$ average nucleotide identity (ANI) to the type genome, which has been assigned the MAG ID CHK191-20366 and which is available via NCBI BioSample SAMN15817035. The GC content of the type genome is $53.29 \%$ and the genome length is 2.3 Mbp.

\section{Description of Candidatus Scatomonas pullistercoris sp. nov.}


8233 Candidatus Scatomonas pullistercoris (pul.li.ster'co.ris. L. masc. n. pullus a young chicken; L.

8234

8235

8236

8237

8238

8239

8240

8241

8242

8243

8244

8245

8246

8247

8248

8249

8250

8251

8252

8253

8254

8255

8256

8257

8258

8259

8260

8261

8262

8263

8264

8265

8266

8267

8268

8269

8270

8271

8272

8273

8274

8275

8276

8277

neut. n. stercus dung; N.L. gen. n. pullistercoris of young chicken faeces)

A bacterial species identified by metagenomic analyses. This species includes all bacteria with genomes that show $\geq 95 \%$ average nucleotide identity (ANI) to the type genome, which has been assigned the MAG ID CHK188-20938 and which is available via NCBI BioSample SAMN15817052. The GC content of the type genome is $53.16 \%$ and the genome length is 2.3 Mbp.

\section{Description of Candidatus Scatomorpha gen. nov.}

Candidatus Scatomorpha (Scat.o.mor'pha. Gr. neut. n. skor, skatos dung; Gr. fem. n. morphe a form, shape; N.L. fem. n. Scatomorpha a microbe associated with faeces)

A bacterial genus identified by metagenomic analyses. The genus includes all bacteria with genomes that show $\geq 60 \%$ average amino acid identity (AAI) to the type genome from the type species Scatomorpha merdavium. This is a name for the alphanumeric GTDB genus UBA5446. This genus has been assigned by GTDB-Tk v1.3.0 working on GTDB Release 05-RS95 (Chaumeil et al., 2019; Parks et al., 2020) to the order Oscillospirales and to the family Oscillospiraceae.

\section{Description of Candidatus Scatomorpha gallistercoris sp. nov.}

Candidatus Scatomorpha gallistercoris (gal.li.ster'co.ris. L. masc. n gallus chicken; L. neut. n. stercus dung; N.L. gen. n. gallistercoris of chicken faeces)

A bacterial species identified by metagenomic analyses. This species includes all bacteria with genomes that show $\geq 95 \%$ average nucleotide identity (ANI) to the type genome, which has been assigned the MAG ID ChiHjej12B11-5383 and which is available via NCBI BioSample SAMN15817213. This is a new name for the alphanumeric GTDB species sp900544765. The $\mathrm{GC}$ content of the type genome is $60.13 \%$ and the genome length is $2.4 \mathrm{Mbp}$.

\section{Description of Candidatus Scatomorpha intestinavium sp. nov.}

Candidatus Scatomorpha intestinavium (in.tes.tin.a'vi.um. L. neut. n. intestinum gut; L. fem. n. avis bird; N.L. gen. n. intestinavium of the gut of birds)

A bacterial species identified by metagenomic analyses. This species includes all bacteria with genomes that show $\geq 95 \%$ average nucleotide identity (ANI) to the type genome, which has been assigned the MAG ID ChiBcolR7-354 and which is available via NCBI BioSample SAMN15817058. The GC content of the type genome is $62.47 \%$ and the genome length is 2.0 Mbp.

\section{Description of Candidatus Scatomorpha intestinigallinarum sp. nov.}


8278 Candidatus Scatomorpha intestinigallinarum (in.tes.ti.ni.gal.li.na'rum. L. neut. n. intestinum gut;

8279 L. fem. n. gallina hen; N.L. gen. n. intestinigallinarum of the gut of the hens)

8280

8281

8282

8283

8284

8285

8286

8287

8288

8289

8290

8291

8292

8293

8294

8295

8296

8297

8298

8299

Description of Candidatus Scatomorpha merdavium sp. nov.

8300 Candidatus Scatomorpha merdavium (merd.a'vi.um. L. fem. n. merda faeces; L. fem. n. avis

8301 bird; N.L. gen. n. merdavium of bird faeces)

8302

8303

8304

8305

8306

8307

8308

8309

8310

8311

A bacterial species identified by metagenomic analyses. This species includes all bacteria with genomes that show $\geq 95 \%$ average nucleotide identity (ANI) to the type genome, which has been assigned the MAG ID ChiSxjej3B15-13231 and which is available via NCBI BioSample SAMN15817220. This is a new name for the alphanumeric GTDB species sp004553625. The $\mathrm{GC}$ content of the type genome is $61.38 \%$ and the genome length is $2.3 \mathrm{Mbp}$.

8312

8313

8314

8315

8316

8317

8318

8319

8320

8321

8322

Description of Candidatus Scatomorpha merdigallinarum sp. nov.

Candidatus Scatomorpha merdigallinarum (mer.di.gal.li.na'rum. L. fem. n. merda faeces; L. fem. n. gallina hen; N.L. gen. n. merdigallinarum of hen faeces)

A bacterial species identified by metagenomic analyses. This species includes all bacteria with genomes that show $\geq 95 \%$ average nucleotide identity (ANI) to the type genome, which has been assigned the MAG ID CHK187-5235 and which is available via NCBI BioSample SAMN15817028. The GC content of the type genome is $58.23 \%$ and the genome length is 2.3 Mbp.

8323

\section{Description of Candidatus Scatomorpha merdipullorum sp. nov.}

Candidatus Scatomorpha merdipullorum (mer.di.pul.lo'rum. L. fem. n. merda faeces; L. masc. n. pullus a young chicken; N.L. gen. n. merdipullorum of the faeces of young chickens) 
8324

8325

8326

8327

8328

8329

8330

8331

8332

8333

8334

8335

8336

8337

8338

8339

8340

8341

8342

8343

8344

8345

8346

8347

8348

8349

8350

8351

8352

8353

8354

8355

8356

8357

8358

8359

8360

8361

8362

8363

8364

8365

8366

8367

8368

A bacterial species identified by metagenomic analyses. This species includes all bacteria with genomes that show $\geq 95 \%$ average nucleotide identity (ANI) to the type genome, which has been assigned the MAG ID ChiHjej10B9-9673 and which is available via NCBI BioSample

SAMN15817099. The GC content of the type genome is $64.45 \%$ and the genome length is 1.8 Mbp.

\section{Description of Candidatus Scatomorpha pullicola sp. nov.}

Candidatus Scatomorpha pullicola (pul.li'co.la. L. masc. n. pullus a young chicken; L. suff. -cola inhabitant of; N.L. n. pullicola an inhabitant of young chickens)

A bacterial species identified by metagenomic analyses. This species includes all bacteria with genomes that show $\geq 95 \%$ average nucleotide identity (ANI) to the type genome, which has been assigned the MAG ID ChiSjej5B23-7677 and which is available via NCBI BioSample SAMN15817223. This is a new name for the alphanumeric GTDB species sp900543085. The $\mathrm{GC}$ content of the type genome is $62.30 \%$ and the genome length is $2.1 \mathrm{Mbp}$.

\section{Description of Candidatus Scatomorpha pullistercoris sp. nov.}

Candidatus Scatomorpha pullistercoris (pul.li.ster'co.ris. L. masc. n. pullus a young chicken; L. neut. n. stercus dung; N.L. gen. n. pullistercoris of young chicken faeces)

A bacterial species identified by metagenomic analyses. This species includes all bacteria with genomes that show $\geq 95 \%$ average nucleotide identity (ANI) to the type genome, which has been assigned the MAG ID ChiHecec3B27-6122 and which is available via NCBI BioSample SAMN15817222. This is a new name for the alphanumeric GTDB species sp900546615. The $\mathrm{GC}$ content of the type genome is $61.20 \%$ and the genome length is $2.2 \mathrm{Mbp}$.

\section{Description of Candidatus Scatomorpha stercoravium sp. nov.}

Candidatus Scatomorpha stercoravium (ster.cor.a'vi.um. L. neut. n. stercus dung; L. fem. n. avis bird; N.L. gen. n. stercoravium of bird faeces)

A bacterial species identified by metagenomic analyses. This species includes all bacteria with genomes that show $\geq 95 \%$ average nucleotide identity (ANI) to the type genome, which has been assigned the MAG ID ChiHecec3B27-8609 and which is available via NCBI BioSample SAMN15817107. The GC content of the type genome is $64.50 \%$ and the genome length is 2.0 Mbp.

\section{Description of Candidatus Scatomorpha stercorigallinarum sp. nov.}

Candidatus Scatomorpha stercorigallinarum (ster.co.ri.gal.li.na'rum. L. neut. n. stercus dung; L. fem. n. gallina hen; N.L. gen. n. stercorigallinarum of hen faeces) 
8369 A bacterial species identified by metagenomic analyses. This species includes all bacteria with 8370 genomes that show $\geq 95 \%$ average nucleotide identity (ANI) to the type genome, which has been 8371 assigned the MAG ID ChiHjej9B8-2268 and which is available via NCBI BioSample

8372 SAMN15817162. The GC content of the type genome is $64.82 \%$ and the genome length is 2.0

8373 Mbp.

8374

8375

8376

8377

8378

8379

8380

8381

8382

8383

8384

8385

8386

8387

8388

8389

8390

8391

8392

8393

8394

8395

8396

8397

8398

8399

8400

8401

8402

8403

8404

8405

8406

8407

8408

8409

8410

8411

8412

8413

8414

\section{Description of Candidatus Scatoplasma gen. nov.}

Candidatus Scatoplasma (Scat.o.plas'ma. Gr. neut. n. skor, skatos dung; Gr. neut. n. plasma a form; N.L. neut. n. Scatoplasma a microbe associated with faeces)

A bacterial genus identified by metagenomic analyses. The genus includes all bacteria with genomes that show $\geq 60 \%$ average amino acid identity (AAI) to the type genome from the type species Scatoplasma merdavium. This is a name for the alphanumeric GTDB genus UBA6879. This genus has been assigned by GTDB-Tk v1.3.0 working on GTDB Release 05-RS95 (Chaumeil et al., 2019; Parks et al., 2020) to the order RFN20 and to the family $C A G-288$.

\section{Description of Candidatus Scatoplasma merdavium sp. nov.}

Candidatus Scatoplasma merdavium (merd.a'vi.um. L. fem. n. merda faeces; L. fem. n. avis bird; N.L. gen. n. merdavium of bird faeces)

A bacterial species identified by metagenomic analyses. This species includes all bacteria with genomes that show $\geq 95 \%$ average nucleotide identity (ANI) to the type genome, which has been assigned the MAG ID 1748 and which is available via NCBI BioSample SAMN15817159. The $\mathrm{GC}$ content of the type genome is $36.99 \%$ and the genome length is $1.0 \mathrm{Mbp}$.

\section{Description of Candidatus Scatosoma gen. nov.}

Candidatus Scatosoma (Scat.o.so'ma. Gr. neut. n. skor, skatos dung; Gr. neut. n. soma a body; N.L. neut. n. Scatosoma a microbe associated with the intestines)

A bacterial genus identified by metagenomic analyses. The genus includes all bacteria with genomes that show $\geq 60 \%$ average amino acid identity (AAI) to the type genome from the type species Scatosoma pullicola. This is a name for the alphanumeric GTDB genus QALS01. This genus has been assigned by GTDB-Tk v1.3.0 working on GTDB Release 05-RS95 (Chaumeil et al., 2019; Parks et al., 2020) to the order Christensenellales and to the family Borkfalkiaceae.

\section{Description of Candidatus Scatosoma pullicola sp. nov.}

Candidatus Scatosoma pullicola (pul.li'co.la. L. masc. n. pullus a young chicken; L. suff. -cola inhabitant of; N.L. n. pullicola an inhabitant of young chickens)

A bacterial species identified by metagenomic analyses. This species includes all bacteria with genomes that show $\geq 95 \%$ average nucleotide identity (ANI) to the type genome, which has been assigned the MAG ID CHK183-20193 and which is available via NCBI BioSample 
8415 SAMN15817013. The GC content of the type genome is $57.19 \%$ and the genome length is 2.0

$8416 \mathrm{Mbp}$.

8417

8418

8419

Description of Candidatus Scatosoma pullistercoris sp. nov.

8420

Candidatus Scatosoma pullistercoris (pul.li.ster'co.ris. L. masc. n. pullus a young chicken; L.

8421

8422

8423 neut. n. stercus dung; N.L. gen. n. pullistercoris of young chicken faeces)

8424

8425

A bacterial species identified by metagenomic analyses. This species includes all bacteria with genomes that show $\geq 95 \%$ average nucleotide identity (ANI) to the type genome, which has been assigned the MAG ID 11687 and which is available via NCBI BioSample SAMN15817138. The

8426

8427

8428

8429 $\mathrm{GC}$ content of the type genome is $55.35 \%$ and the genome length is $1.5 \mathrm{Mbp}$.

8430

\section{Description of Candidatus Scatousia gen. nov.}

Candidatus Scatousia (Scat.ou'si.a. Gr. neut. n. skor, skatos dung; Gr. fem. n. ousia an essence;

8431

8432

8433 N.L. fem. n. Scatousia a microbe associated with faeces)

8434

8435

A bacterial genus identified by metagenomic analyses. The genus includes all bacteria with genomes that show $\geq 60 \%$ average amino acid identity (AAI) to the type genome from the type

8436

8437

8438 species Scatousia excrementipullorum. This is a name for the alphanumeric GTDB genus CAG484. This genus has been assigned by GTDB-Tk v1.3.0 working on GTDB Release 05-RS95 (Chaumeil et al., 2019; Parks et al., 2020) to the order Gastranaerophilales and to the family Gastranaerophilaceae.

8439

8440

8441

Description of Candidatus Scatousia excrementigallinarum sp. nov.

8442 Candidatus Scatousia excrementigallinarum (ex.cre.men.ti.gal.li.na'rum. L. neut. n. excrementum excrement; L. fem. n. gallina hen; N.L. gen. n. excrementigallinarum of hen excrement)

8444

8445

A bacterial species identified by metagenomic analyses. This species includes all bacteria with 8446 genomes that show $\geq 95 \%$ average nucleotide identity (ANI) to the type genome, which has been assigned the MAG ID 6276 and which is available via NCBI BioSample SAMN15817091. The $\mathrm{GC}$ content of the type genome is $36.46 \%$ and the genome length is $2.8 \mathrm{Mbp}$.

\section{Description of Candidatus Scatousia excrementipullorum sp. nov.}

8452 Candidatus Scatousia excrementipullorum (ex.cre.men.ti.pul.lo'rum. L. neut. n. excrementum excrement; L. masc. n. pullus a young chicken; N.L. gen. n. excrementipullorum of young chicken excrement)

8455

8456

8457

A bacterial species identified by metagenomic analyses. This species includes all bacteria with genomes that show $\geq 95 \%$ average nucleotide identity (ANI) to the type genome, which has been

8459 assigned the MAG ID 10192 and which is available via NCBI BioSample SAMN15817157. The 8460 $\mathrm{GC}$ content of the type genome is $36.06 \%$ and the genome length is $1.8 \mathrm{Mbp}$. 
Description of Candidatus Scatovicinus gen. nov.

8464 Candidatus Scatovicinus (Scat.o.vic.in'us. Gr. neut. n. skor, skatos dung; L. masc. n. vicinus a neighbour; N.L. masc. n. Scatovicinus a microbe associated with faeces)

A bacterial genus identified by metagenomic analyses. The genus includes all bacteria with genomes that show $\geq 60 \%$ average amino acid identity (AAI) to the type genome from the type species Scatovicinus merdipullorum. This is a name for the alphanumeric GTDB genus UMGS403. This genus has been assigned by GTDB-Tk v1.3.0 working on GTDB Release 05RS95 (Chaumeil et al., 2019; Parks et al., 2020) to the order Oscillospirales and to the family Acutalibacteraceae.

8473

8474

8475

\section{Description of Candidatus Scatovicinus merdipullorum sp. nov.}

8476

Candidatus Scatovicinus merdipullorum (mer.di.pul.lo'rum. L. fem. n. merda faeces; L. masc. $\mathrm{n}$.

8477

8478

pullus a young chicken; N.L. gen. n. merdipullorum of the faeces of young chickens)

8479

A bacterial species identified by metagenomic analyses. This species includes all bacteria with genomes that show $\geq 95 \%$ average nucleotide identity (ANI) to the type genome, which has been

8481 assigned the MAG ID CHK181-9830 and which is available via NCBI BioSample

8482 SAMN15817214. This is a new name for the alphanumeric GTDB species sp900541565. The

8483

8484 $\mathrm{GC}$ content of the type genome is $46.80 \%$ and the genome length is $1.9 \mathrm{Mbp}$.

\section{Description of Candidatus Scatovivens gen. nov.}

8487 Candidatus Scatovivens (Scat.o.viv'ens. Gr. neut. n. skor, skatos dung; N.L. pres. part. vivens living; N.L. fem. n. Scatovivens a microbe associated with faeces)

8490

A bacterial genus identified by metagenomic analyses. The genus includes all bacteria with genomes that show $\geq 60 \%$ average amino acid identity (AAI) to the type genome from the type species Scatovivens faecipullorum. This is a name for the alphanumeric GTDB genus UBA7001. This genus has been assigned by GTDB-Tk v1.3.0 working on GTDB Release 05-RS95 (Chaumeil et al., 2019; Parks et al., 2020) to the order TANB77 and to the family CAG-508.

\section{Description of Candidatus Scatovivens faecipullorum sp. nov.}

8497 Candidatus Scatovivens faecipullorum (fae.ci.pul.lo'rum. L. fem. n. faex, faecis excrement; L. masc. n. pullus a young chicken; N.L. gen. n. faecipullorum of young chicken faeces)

A bacterial species identified by metagenomic analyses. This species includes all bacteria with genomes that show $\geq 95 \%$ average nucleotide identity (ANI) to the type genome, which has been assigned the MAG ID ChiSxjej5B17-16517 and which is available via NCBI BioSample

8503 SAMN15817189. This is a new name for the alphanumeric GTDB species sp900553685. The $8504 \mathrm{GC}$ content of the type genome is $25.63 \%$ and the genome length is $1.9 \mathrm{Mbp}$. 
8507 Description of Candidatus Scubalenecus merdavium sp. nov.

8508 Candidatus Scubalenecus merdavium (merd.a'vi.um. L. fem. n. merda faeces; L. fem. n. avis

8509 bird; N.L. gen. n. merdavium of bird faeces)

8510

8511 A bacterial species identified by metagenomic analyses. This species includes all bacteria with

8512 genomes that show $\geq 95 \%$ average nucleotide identity (ANI) to the type genome, which has been

8513 assigned the MAG ID CHK176-6737 and which is available via NCBI BioSample

8514 SAMN15817203. This is a new name for the alphanumeric GTDB species sp900546735. The

8515 GC content of the type genome is $52.55 \%$ and the genome length is $1.8 \mathrm{Mbp}$.

8516

8517

8518 Description of Candidatus Scubalocola faecavium sp. nov.

8519 Candidatus Scubalocola faecavium (faec.a'vi.um. L. fem. n. faex, faecis excrement; L. fem. n.

8520 avis bird; N.L. gen. n. faecavium of bird faeces)

8521

8522

A bacterial species identified by metagenomic analyses. This species includes all bacteria with

8523

8524

8525

8526

8527

8528

8529

Description of Candidatus Scubalocola faecigallinarum sp. nov.

8530 Candidatus Scubalocola faecigallinarum (fae.ci.gal.li.na'rum. L. fem. n. faex, faecis excrement;

8531

L. fem. n. gallina hen; N.L. gen. n. faecigallinarum of hen faeces)

8532

8533

8534

8535

A bacterial species identified by metagenomic analyses. This species includes all bacteria with genomes that show $\geq 95 \%$ average nucleotide identity (ANI) to the type genome, which has been assigned the MAG ID CHK178-757 and which is available via NCBI BioSample

8536 SAMN15817027. The GC content of the type genome is $46.78 \%$ and the genome length is 3.5

8537 Mbp.

8538

8539

8540

Description of Candidatus Scubalocola faecipullorum sp. nov.

8541 Candidatus Scubalocola faecipullorum (fae.ci.pul.lo'rum. L. fem. n. faex, faecis excrement; L.

8542

8543

8544

8545

masc. n. pullus a young chicken; N.L. gen. n. faecipullorum of young chicken faeces)

8546

A bacterial species identified by metagenomic analyses. This species includes all bacteria with genomes that show $\geq 95 \%$ average nucleotide identity (ANI) to the type genome, which has been

8547 assigned the MAG ID CHK194-7924 and which is available via NCBI BioSample

8548 SAMN15817034. The GC content of the type genome is $44.36 \%$ and the genome length is 2.5

8549

8550

8551

Description of Candidatus Scubalomonas excrementavium sp. nov.

Peer) reviewing PDF | (2020:12:55980:1:1:NEW 13 Jan 2021) 
8552 Candidatus Scubalomonas excrementavium (ex.cre.ment.a'vi.um. L. neut. n. excrementum

8553

8554

8555

8556

8557

8558

8559

8560

8561

8562

8563

8564

8565

8566

8567

8568

8569

8570

8571

8572

8573

8574

8575

8576

8577

8578

8579

8580

8581

8582

8583

8584

8585

8586

8587

8588

8589

8590

8591

8592

8593

8594

8595

8596

8597

excrement; L. fem. n. avis bird; N.L. gen. n. excrementavium of bird excrement)

A bacterial species identified by metagenomic analyses. This species includes all bacteria with genomes that show $\geq 95 \%$ average nucleotide identity (ANI) to the type genome, which has been assigned the MAG ID E3-2379 and which is available via NCBI BioSample SAMN15817094. The GC content of the type genome is $32.78 \%$ and the genome length is $2.5 \mathrm{Mbp}$.

\section{Description of Candidatus Scubalomonas excrementigallinarum sp. nov.}

Candidatus Scubalomonas excrementigallinarum (ex.cre.men.ti.gal.li.na'rum. L. neut. n. excrementum excrement; L. fem. n. gallina hen; N.L. gen. n. excrementigallinarum of hen excrement)

A bacterial species identified by metagenomic analyses. This species includes all bacteria with genomes that show $\geq 95 \%$ average nucleotide identity (ANI) to the type genome, which has been assigned the MAG ID 3201 and which is available via NCBI BioSample SAMN15817095. The $\mathrm{GC}$ content of the type genome is $34.32 \%$ and the genome length is $3.2 \mathrm{Mbp}$.

\section{Description of Candidatus Scubalosoma faecavium sp. nov.}

Candidatus Scubalosoma faecavium (faec.a'vi.um. L. fem. n. faex, faecis excrement; L. fem. n. avis bird; N.L. gen. n. faecavium of bird faeces)

A bacterial species identified by metagenomic analyses. This species includes all bacteria with genomes that show $\geq 95 \%$ average nucleotide identity (ANI) to the type genome, which has been assigned the MAG ID ChiSxjej1B13-2233 and which is available via NCBI BioSample SAMN15817224. This is a new name for the alphanumeric GTDB species sp900545085. The $\mathrm{GC}$ content of the type genome is $47.85 \%$ and the genome length is $1.6 \mathrm{Mbp}$.

\section{Description of Candidatus Scubalousia intestinigallinarum sp. nov.}

Candidatus Scubalousia intestinigallinarum (in.tes.ti.ni.gal.li.na'rum. L. neut. n. intestinum gut; L. fem. n. gallina hen; N.L. gen. n. intestinigallinarum of the gut of the hens)

A bacterial species identified by metagenomic analyses. This species includes all bacteria with genomes that show $\geq 95 \%$ average nucleotide identity (ANI) to the type genome, which has been assigned the MAG ID CHK193-12526 and which is available via NCBI BioSample SAMN15817037. The GC content of the type genome is $31.54 \%$ and the genome length is 1.5 Mbp.

\section{Description of Candidatus Scybalenecus gen. nov.}

Candidatus Scybalenecus (Scy.bal.en.e'cus. Gr. neut. n. scybalon dung; Gr. masc. enoikos inhabitant; N.L. masc. n. Scybalenecus a microbe associated with faeces) 
8598 A bacterial genus identified by metagenomic analyses. The genus includes all bacteria with 8599 genomes that show $\geq 60 \%$ average amino acid identity (AAI) to the type genome from the type 8600 species Scybalenecus merdavium. This is a name for the alphanumeric GTDB genus UMGS905. 8601 This genus has been assigned by GTDB-Tk v1.3.0 working on GTDB Release 05-RS95 8602 (Chaumeil et al., 2019; Parks et al., 2020) to the order Oscillospirales and to the family 8603 Acutalibacteraceae.

8604

8605

8606

8607

Description of Candidatus Scybalocola gen. nov.

8608

8609

8610

Candidatus Scybalocola (Scy.bal.o.co'la. Gr. neut. n. scybalon dung; L. suff. -cola inhabitant of; N.L. fem. n. Scybalocola a microbe associated with the intestines)

8611

8612

A bacterial genus identified by metagenomic analyses. The genus includes all bacteria with genomes that show $\geq 60 \%$ average amino acid identity (AAI) to the type genome from the type

8613 species Scybalocola faecigallinarum. This is a name for the alphanumeric GTDB genus

8614 UBA7096. This genus has been assigned by GTDB-Tk v1.3.0 working on GTDB Release 05-

8615 RS95 (Chaumeil et al., 2019; Parks et al., 2020) to the order Lachnospirales and to the family

8616

8617 Lachnospiraceae.

8619

Description of Candidatus Scybalomonas gen. nov.

8620

8621

8622

8623

8624 Candidatus Scybalomonas (Scy.bal.omo'nas. Gr. neut. n. scybalon dung; L. fem. n. monas a monad; N.L. fem. n. Scybalomonas a microbe associated with faeces)

A bacterial genus identified by metagenomic analyses. The genus includes all bacteria with genomes that show $\geq 60 \%$ average amino acid identity (AAI) to the type genome from the type species Scybalomonas excrementigallinarum. This is a name for the alphanumeric GTDB genus UMGS680. This genus has been assigned by GTDB-Tk v1.3.0 working on GTDB Release 05RS95 (Chaumeil et al., 2019; Parks et al., 2020) to the order Lachnospirales and to the family

8627 Lachnospiraceae.

8628

8629

8630

8631

Description of Candidatus Scybalosoma gen. nov.

8632

Candidatus Scybalosoma (Scy.bal.o.so'ma. Gr. neut. n. scybalon dung; Gr. neut. n. soma a body;

8633

8634 N.L. neut. n. Scybalosoma a microbe associated with faeces)

8635

A bacterial genus identified by metagenomic analyses. The genus includes all bacteria with

8636 genomes that show $\geq 60 \%$ average amino acid identity (AAI) to the type genome from the type

8637 species Scybalosoma faecavium. This is a name for the alphanumeric GTDB genus UMGS743.

8638 This genus has been assigned by GTDB-Tk v1.3.0 working on GTDB Release 05-RS95

8639 (Chaumeil et al., 2019; Parks et al., 2020) to the order Christensenellales and to the family

8640

8641 Christensenellaceae.

\section{Description of Candidatus Scybalousia gen. nov.}


8643 Candidatus Scybalousia (Scy.bal.ou'si.a. Gr. neut. n. scybalon dung; Gr. fem. n. ousia an

8644

8645

8646

8647

8648

8649

8650

8651

8652

8653

8654

8655

8656

8657

8658

8659

8660

8661

8662

8663

8664

8665

8666

8667

8668

8669

8670

8671

8672

8673

8674

8675

8676

8677

8678

8679

8680

8681

8682

8683

8684

8685

8686

8687

8688

essence; N.L. fem. n. Scybalousia a microbe associated with faeces)

A bacterial genus identified by metagenomic analyses. The genus includes all bacteria with genomes that show $\geq 60 \%$ average amino acid identity (AAI) to the type genome from the type species Scybalousia intestinigallinarum. This is a name for the alphanumeric GTDB genus UBA7057. This genus has been assigned by GTDB-Tk v1.3.0 working on GTDB Release 05RS95 (Chaumeil et al., 2019; Parks et al., 2020) to the order RF39 and to the family CAG-611.

\section{Description of Candidatus Sellimonas avistercoris sp. nov.}

Candidatus Sellimonas avistercoris (a.vi.ster'co.ris. L. fem. n. avis bird; L. neut. n. stercus dung; N.L. gen. n. avistercoris of bird faeces)

A bacterial species identified by metagenomic analyses. This species includes all bacteria with genomes that show $\geq 95 \%$ average nucleotide identity (ANI) to the type genome, which has been assigned the MAG ID ChiBcec13-3606 and which is available via NCBI BioSample

SAMN15816877. This is a new name for the alphanumeric GTDB species sp002161525. The $\mathrm{GC}$ content of the type genome is $46.79 \%$ and the genome length is $2.6 \mathrm{Mbp}$.

\section{Description of Candidatus Sphingobacterium stercorigallinarum sp. nov.}

Candidatus Sphingobacterium stercorigallinarum (ster.co.ri.gal.li.na'rum. L. neut. n. stercus dung; L. fem. n. gallina hen; N.L. gen. n. stercorigallinarum of hen faeces)

A bacterial species identified by metagenomic analyses. This species includes all bacteria with genomes that show $\geq 95 \%$ average nucleotide identity (ANI) to the type genome, which has been assigned the MAG ID CHK174-1108 and which is available via NCBI BioSample SAMN15816705. The GC content of the type genome is $44.57 \%$ and the genome length is 2.9 Mbp.

\section{Description of Candidatus Sphingobacterium stercoripullorum sp. nov.} Candidatus Sphingobacterium stercoripullorum (ster.co.ri.pul.lo'rum. L. neut. n. stercus dung; L. masc. n. pullus a young chicken; N.L. gen. n. stercoripullorum of the faceces of young chickens)

A bacterial species identified by metagenomic analyses. This species includes all bacteria with genomes that show $\geq 95 \%$ average nucleotide identity (ANI) to the type genome, which has been assigned the MAG ID 1719 and which is available via NCBI BioSample SAMN15816733. The $\mathrm{GC}$ content of the type genome is $39.36 \%$ and the genome length is $2.7 \mathrm{Mbp}$.

\section{Description of Candidatus Sphingomonas excrementigallinarum sp. nov.}

Candidatus Sphingomonas excrementigallinarum (ex.cre.men.ti.gal.li.na'rum. L. neut. n. excrementum excrement; L. fem. n. gallina hen; N.L. gen. n. excrementigallinarum of hen excrement) 
8689

8690

8691

8692

8693

8694

8695

8696

8697

8698

8699

8700

8701

8702

8703

8704

8705

8706

8707

8708

8709

8710

8711

8712

8713

8714

8715

8716

8717

8718

8719

8720

8721

8722

8723

8724

8725

8726

8727

8728

8729

8730

8731

8732

8733

8734

A bacterial species identified by metagenomic analyses. This species includes all bacteria with genomes that show $\geq 95 \%$ average nucleotide identity (ANI) to the type genome, which has been assigned the MAG ID 1562 and which is available via NCBI BioSample SAMN15816779. The $\mathrm{GC}$ content of the type genome is $66.49 \%$ and the genome length is $3.7 \mathrm{Mbp}$.

\section{Description of Candidatus Spyradenecus gen. nov.}

Candidatus Spyradenecus (Spy.rad.en.e'cus. Gr. fem. n. spyras ball of dung; Gr. masc. enoikos inhabitant; N.L. masc. n. Spyradenecus a microbe associated with the intestines)

A bacterial genus identified by metagenomic analyses. The genus includes all bacteria with genomes that show $\geq 60 \%$ average amino acid identity (AAI) to the type genome from the type species Spyradenecus faecavium. This is a name for the alphanumeric GTDB genus W1P29-020. This genus has been assigned by GTDB-Tk v1.3.0 working on GTDB Release 05-RS95 (Chaumeil et al., 2019; Parks et al., 2020) to the order RFP12 and to the family W1P29-020.

\section{Description of Candidatus Spyradenecus faecavium sp. nov.}

Candidatus Spyradenecus faecavium (faec.a'vi.um. L. fem. n. faex, faecis excrement; L. fem. n. avis bird; N.L. gen. n. faecavium of bird faeces)

A bacterial species identified by metagenomic analyses. This species includes all bacteria with genomes that show $\geq 95 \%$ average nucleotide identity (ANI) to the type genome, which has been assigned the MAG ID 35461 and which is available via NCBI BioSample SAMN15817160. The $\mathrm{GC}$ content of the type genome is $68.44 \%$ and the genome length is $1.8 \mathrm{Mbp}$.

\section{Description of Candidatus Spyradocola gen. nov.}

Candidatus Spyradocola (Spy.rad.o.co'la. Gr. fem. n. spyras ball of dung; L. suff. -cola inhabitant of; N.L. fem. n. Spyradocola a microbe associated with the intestines)

A bacterial genus identified by metagenomic analyses. The genus includes all bacteria with genomes that show $\geq 60 \%$ average amino acid identity (AAI) to the type genome from the type species Spyradocola merdavium. This is a name for the alphanumeric GTDB genus UBA7102. This genus has been assigned by GTDB-Tk v1.3.0 working on GTDB Release 05-RS95 (Chaumeil et al., 2019; Parks et al., 2020) to the order Christensenellales and to the family UBA1750.

\section{Description of Candidatus Spyradocola merdavium sp. nov.}

Candidatus Spyradocola merdavium (merd.a'vi.um. L. fem. n. merda faeces; L. fem. n. avis bird; N.L. gen. n. merdavium of bird faeces)

A bacterial species identified by metagenomic analyses. This species includes all bacteria with genomes that show $\geq 95 \%$ average nucleotide identity (ANI) to the type genome, which has been

Peer) reviewing PDF | (2020:12:55980:1:1:NEW 13 Jan 2021) 
8735 assigned the MAG ID CHK191-18038 and which is available via NCBI BioSample

8736 SAMN15817024. The GC content of the type genome is $66.20 \%$ and the genome length is 2.6

8737 Mbp.

8738

8739

8740

8741

8742

8743

8744

8745

8746

8747

8748

8749

8750

8751

8752

8753

8754

8755

8756

8757

8758

8759

\section{Description of Candidatus Spyradomonas gen. nov.}

Candidatus Spyradomonas (Spy.rad.omo'nas. Gr. fem. n. spyras ball of dung; L. fem. n. monas a monad; N.L. fem. n. Spyradomonas a microbe associated with faeces)

A bacterial genus identified by metagenomic analyses. The genus includes all bacteria with genomes that show $\geq 60 \%$ average amino acid identity (AAI) to the type genome from the type species Spyradomonas excrementavium. This is a name for the alphanumeric GTDB genus UMGS951. This genus has been assigned by GTDB-Tk v1.3.0 working on GTDB Release 05RS95 (Chaumeil et al., 2019; Parks et al., 2020) to the order Gastranaerophilales and to the family Gastranaerophilaceae.

\section{Description of Candidatus Spyradomonas excrementavium sp. nov.}

Candidatus Spyradomonas excrementavium (ex.cre.ment.a'vi.um. L. neut. n. excrementum excrement; L. fem. n. avis bird; N.L. gen. n. excrementavium of bird excrement)

A bacterial species identified by metagenomic analyses. This species includes all bacteria with genomes that show $\geq 95 \%$ average nucleotide identity (ANI) to the type genome, which has been assigned the MAG ID CHK149-2741 and which is available via NCBI BioSample

8760 SAMN15817232. This is a new name for the alphanumeric GTDB species sp900547155. The $\mathrm{GC}$ content of the type genome is $41.54 \%$ and the genome length is $2.0 \mathrm{Mbp}$.

8761

8762

8763

\section{Description of Candidatus Spyradosoma gen. nov.}

8764 Candidatus Spyradosoma (Spy.rad.o.so'ma. Gr. fem. n. spyras ball of dung; Gr. neut. n. soma a

8765

8766

8767

8768

8769 body; N.L. neut. n. Spyradosoma a microbe associated with faeces)

8770

8771

8772

8773

8774

8775

A bacterial genus identified by metagenomic analyses. The genus includes all bacteria with genomes that show $\geq 60 \%$ average amino acid identity (AAI) to the type genome from the type species Spyradosoma merdigallinarum. This is a name for the alphanumeric GTDB genus W0P29-029. This genus has been assigned by GTDB-Tk v1.3.0 working on GTDB Release 05RS95 (Chaumeil et al., 2019; Parks et al., 2020) to the order Opitutales and to the family UBA953.

8776

8777

8778

8779

Description of Candidatus Spyradosoma merdigallinarum sp. nov. Candidatus Spyradosoma merdigallinarum (mer.di.gal.li.na'rum. L. fem. n. merda faeces; L. fem. n. gallina hen; N.L. gen. n. merdigallinarum of hen faeces)

8780

A bacterial species identified by metagenomic analyses. This species includes all bacteria with genomes that show $\geq 95 \%$ average nucleotide identity (ANI) to the type genome, which has been 
8781 assigned the MAG ID 10669 and which is available via NCBI BioSample SAMN15817156. The

8782

8783

8784

8785

8786

8787

8788

8789

8790

8791

8792

8793

8794

8795

8796

8797

8798

8799

8800

8801

8802

8803

8804

8805

8806

8807

8808

8809

8810

8811

8812

8813

8814

8815

8816

8817

8818

8819

8820

8821

8822

8823

8824

8825

$\mathrm{GC}$ content of the type genome is $62.43 \%$ and the genome length is $1.6 \mathrm{Mbp}$.

\section{Description of Candidatus Spyradousia gen. nov.}

Candidatus Spyradousia (Spy.rad.ou'si.a. Gr. fem. n. spyras ball of dung; Gr. fem. n. ousia an essence; N.L. fem. n. Spyradousia a microbe associated with the intestines)

A bacterial genus identified by metagenomic analyses. The genus includes all bacteria with genomes that show $\geq 60 \%$ average amino acid identity (AAI) to the type genome from the type species Spyradousia avicola. This is a name for the alphanumeric GTDB genus UBA71. This genus has been assigned by GTDB-Tk v1.3.0 working on GTDB Release 05-RS95 (Chaumeil et al., 2019; Parks et al., 2020) to the order Methanomassiliicoccales and to the family Methanomethylophilaceae.

\section{Description of Candidatus Spyradousia avicola sp. nov.}

Candidatus Spyradousia avicola (a.vi'co.la. L. fem. n. avis bird; L. suff. -cola inhabitant of; N.L. n. avicola inhabitant of birds)

A bacterial species identified by metagenomic analyses. This species includes all bacteria with genomes that show $\geq 95 \%$ average nucleotide identity (ANI) to the type genome, which has been assigned the MAG ID 6227 and which is available via NCBI BioSample SAMN15817164. The $\mathrm{GC}$ content of the type genome is $60.22 \%$ and the genome length is $1.5 \mathrm{Mbp}$.

\section{Description of Candidatus Stackebrandtia excrementipullorum sp. nov.}

Candidatus Stackebrandtia excrementipullorum (ex.cre.men.ti.pul.lo'rum. L. neut. n.

excrementum excrement; L. masc. n. pullus a young chicken; N.L. gen. n. excrementipullorum of young chicken excrement)

A bacterial species identified by metagenomic analyses. This species includes all bacteria with genomes that show $\geq 95 \%$ average nucleotide identity (ANI) to the type genome, which has been assigned the MAG ID ChiHjej8B7-33794 and which is available via NCBI BioSample SAMN15816761. The GC content of the type genome is $64.17 \%$ and the genome length is 4.1 Mbp.

\section{Description of Candidatus Stackebrandtia faecavium sp. nov.}

Candidatus Stackebrandtia faecavium (faec.a'vi.um. L. fem. n. faex, faecis excrement; L. fem. n. avis bird; N.L. gen. n. faecavium of bird faeces)

A bacterial species identified by metagenomic analyses. This species includes all bacteria with genomes that show $\geq 95 \%$ average nucleotide identity ( $\mathrm{ANI}$ ) to the type genome, which has been assigned the MAG ID ChiGjej4B4-770 and which is available via NCBI BioSample 
8826 SAMN15816782. The GC content of the type genome is $62.84 \%$ and the genome length is 3.4

8827 Mbp.

8828

8829

8830

Description of Candidatus Stercoripulliclostridium gen. nov.

8831

Candidatus Stercoripulliclostridium (Ster.co.ri.pul.li.clos.tri'di.um. L. neut. n. stercus dung; N.L.

8832 masc. n. pullus a young chicken; N.L. neut. n. Clostridium a genus name; N.L. neut. n.

8833

8834

8835 Stercoripulliclostridium a genus related to the genus Clostridium but distinct from it and found in poultry faeces)

8836

8837

A bacterial genus identified by metagenomic analyses. The genus includes all bacteria with

8838 genomes that show $\geq 60 \%$ average amino acid identity (AAI) to the type genome from the type

8839 species Stercoripulliclostridium merdipullorum. This genus has been assigned by GTDB-Tk

8840 v1.3.0 working on GTDB Release 05-RS95 (Chaumeil et al., 2019; Parks et al., 2020) to the order Christensenellales and to the family DTU072.

8841

8842

8843

8844

8845

8846

8847

8848

8849

Description of Candidatus Stercoripulliclostridium merdigallinarum sp. nov. Candidatus Stercoripulliclostridium merdigallinarum (mer.di.gal.li.na'rum. L. fem. n. merda faeces; L. fem. n. gallina hen; N.L. gen. n. merdigallinarum of hen faeces)

8850

8851

8852

8853

A bacterial species identified by metagenomic analyses. This species includes all bacteria with genomes that show $\geq 95 \%$ average nucleotide identity (ANI) to the type genome, which has been assigned the MAG ID 18911 and which is available via NCBI BioSample SAMN15816986. The $\mathrm{GC}$ content of the type genome is $48.67 \%$ and the genome length is $1.5 \mathrm{Mbp}$.

8854

Description of Candidatus Stercoripulliclostridium merdipullorum sp. nov.

Candidatus Stercoripulliclostridium merdipullorum (mer.di.pul.lo'rum. L. fem. n. merda faeces;

8855

L. masc. $\mathrm{n}$. pullus a young chicken; N.L. gen. n. merdipullorum of the faeces of young chickens)

8856

8857

8858

A bacterial species identified by metagenomic analyses. This species includes all bacteria with

8859 genomes that show $\geq 95 \%$ average nucleotide identity (ANI) to the type genome, which has been

8860 assigned the MAG ID 23406 and which is available via NCBI BioSample SAMN15816995. The

8861

8862

8863 $\mathrm{GC}$ content of the type genome is $53.33 \%$ and the genome length is $1.7 \mathrm{Mbp}$.

8864

Description of Candidatus Stercoripulliclostridium pullicola sp. nov.

8865

Candidatus Stercoripulliclostridium pullicola (pul.li'co.la. L. masc. n. pullus a young chicken; L.

8866

8867 A bacterial species identified by metagenomic analyses. This species includes all bacteria with 8868 genomes that show $\geq 95 \%$ average nucleotide identity (ANI) to the type genome, which has been 8869

8870 assigned the MAG ID 517 and which is available via NCBI BioSample SAMN15816997. The 8871 $\mathrm{GC}$ content of the type genome is $52.41 \%$ and the genome length is $1.6 \mathrm{Mbp}$. 
8872

8873

8875

8876

8877

8878

8879

8880

8881

8882

8883

8884

8885

8886

8887

8888

8889

8890

8891

8892

8893

8894

8895

8896

8897

8898

8899

8900

8901

8902

8903

8904

8905

8906

8907

8908

8909

8910

8911

8912

8913

8914

8915

8916

8917

\section{Description of Candidatus Stercorousia gen. nov.}

Candidatus Stercorousia (Ster.cor.ou'si.a. L. neut. n. stercus dung; Gr. fem. n. ousia an essence; N.L. fem. n. Stercorousia a microbe associated with the intestines)

A bacterial genus identified by metagenomic analyses. The genus includes all bacteria with genomes that show $\geq 60 \%$ average amino acid identity (AAI) to the type genome from the type species Stercorousia faecigallinarum. This is a name for the alphanumeric GTDB genus Zag1. This genus has been assigned by GTDB-Tk v1.3.0 working on GTDB Release 05-RS95 (Chaumeil et al., 2019; Parks et al., 2020) to the order Gastranaerophilales and to the family Gastranaerophilaceae.

\section{Description of Candidatus Stercorousia faecigallinarum sp. nov.}

Candidatus Stercorousia faecigallinarum (fae.ci.gal.li.na'rum. L. fem. n. faex, faecis excrement; L. fem. n. gallina hen; N.L. gen. n. faecigallinarum of hen faeces)

A bacterial species identified by metagenomic analyses. This species includes all bacteria with genomes that show $\geq 95 \%$ average nucleotide identity (ANI) to the type genome, which has been assigned the MAG ID CHK154-323 and which is available via NCBI BioSample

SAMN15817192. This is a new name for the alphanumeric GTDB species sp000438175. The $\mathrm{GC}$ content of the type genome is $34.85 \%$ and the genome length is $2.1 \mathrm{Mbp}$.

\section{Description of Candidatus Streptococcus faecavium sp. nov.}

Candidatus Streptococcus faecavium (faec.a'vi.um. L. fem. n. faex, faecis excrement; L. fem. n. avis bird; N.L. gen. n. faecavium of bird faeces)

A bacterial species identified by metagenomic analyses. This species includes all bacteria with genomes that show $\geq 95 \%$ average nucleotide identity (ANI) to the type genome, which has been assigned the MAG ID ChiBcolR9-63 and which is available via NCBI BioSample

SAMN15816845. This is a new name for the alphanumeric GTDB species sp002300045. The $\mathrm{GC}$ content of the type genome is $40.90 \%$ and the genome length is $1.4 \mathrm{Mbp}$.

\section{Description of Candidatus Sutterella merdavium sp. nov.}

Candidatus Sutterella merdavium (merd.a'vi.um. L. fem. n. merda faeces; L. fem. n. avis bird; N.L. gen. n. merdavium of bird faeces)

A bacterial species identified by metagenomic analyses. This species includes all bacteria with genomes that show $\geq 95 \%$ average nucleotide identity (ANI) to the type genome, which has been assigned the MAG ID ChiGjej6B6-11950 and which is available via NCBI BioSample SAMN15816882. This is a new name for the alphanumeric GTDB species sp900543805. The $\mathrm{GC}$ content of the type genome is $62.35 \%$ and the genome length is $2.1 \mathrm{Mbp}$. 
8918 Description of Candidatus Tetragenococcus pullicola sp. nov.

8919 Candidatus Tetragenococcus pullicola (pul.li'co.la. L. masc. n. pullus a young chicken; L. suff. 8920 cola inhabitant of; N.L. n. pullicola an inhabitant of young chickens)

8921

8922

8923

8924

8925

8926

8927

8928

8929

Description of Candidatus Tidjanibacter faecipullorum sp. nov.

8930 Candidatus Tidjanibacter faecipullorum (fae.ci.pul.lo'rum. L. fem. n.faex, faecis excrement; L.

8931

8932

8933 masc. n. pullus a young chicken; N.L. gen. n. faecipullorum of young chicken faeces)

A bacterial species identified by metagenomic analyses. This species includes all bacteria with genomes that show $\geq 95 \%$ average nucleotide identity (ANI) to the type genome, which has been assigned the MAG ID CHK175-10598 and which is available via NCBI BioSample SAMN15816709. The GC content of the type genome is $36.35 \%$ and the genome length is 2.6 Mbp.

8934

A bacterial species identified by metagenomic analyses. This species includes all bacteria with genomes that show $\geq 95 \%$ average nucleotide identity (ANI) to the type genome, which has been

8935

8936

8937 assigned the MAG ID ChiHjej11B10-19426 and which is available via NCBI BioSample SAMN15816696. The GC content of the type genome is $60.46 \%$ and the genome length is 1.8 Mbp.

8938

8939

8940

Description of Candidatus Tidjanibacter gallistercoris sp. nov.

8941 Candidatus Tidjanibacter gallistercoris (gal.li.ster'co.ris. L. masc. n. gallus chicken; L. neut. n.

8942

8943

8944 stercus dung; N.L. gen. n. gallistercoris of chicken faeces)

8945

A bacterial species identified by metagenomic analyses. This species includes all bacteria with

8946

8947 genomes that show $\geq 95 \%$ average nucleotide identity (ANI) to the type genome, which has been assigned the MAG ID ChiSxjej4B16-7142 and which is available via NCBI BioSample

8948 Mbp.

8949

8950

8951

\section{Description of Candidatus Treponema excrementipullorum sp. nov.}

8952

Candidatus Treponema excrementipullorum (ex.cre.men.ti.pul.lo'rum. L. neut. n. excrementum

8953 excrement; L. masc. n. pullus a young chicken; N.L. gen. n. excrementipullorum of young

8954

8955

8956 chicken excrement)

8957

A bacterial species identified by metagenomic analyses. This species includes all bacteria with

8958 genomes that show $\geq 95 \%$ average nucleotide identity (ANI) to the type genome, which has been

8959 assigned the MAG ID Gambia15-2214 and which is available via NCBI BioSample

8960 SAMN15816896. Although GTDB has assigned this species to the genus it calls Treponema_F , this genus designation cannot be incorporated into a well-formed binomial, so in naming this

8962 species, we have used the current validly published name for the genus. The GC content of the 8963 type genome is $39.91 \%$ and the genome length is $2.3 \mathrm{Mbp}$. 
8964

8965

8966

8967

8968

8969

8970

8971

8972

8973

8974

8975

8976

8977

8978

8979

8980

8981

8982

8983

8984

8985

8986

8987

8988

8989

8990

8991

8992

8993

8994

8995

8996

8997

8998

8999

9000

9001

9002

9003

9004

9005

9006

9007

9008

9009

\section{Description of Candidatus Treponema faecavium sp. nov.}

Candidatus Treponema faecavium (faec.a'vi.um. L. fem. n. faex, faecis excrement; L. fem. n. avis bird; N.L. gen. n. faecavium of bird faeces)

A bacterial species identified by metagenomic analyses. This species includes all bacteria with genomes that show $\geq 95 \%$ average nucleotide identity (ANI) to the type genome, which has been assigned the MAG ID USASDec8-330 and which is available via NCBI BioSample SAMN15816910. Although GTDB has assigned this species to the genus it calls Treponema_F , this genus designation cannot be incorporated into a well-formed binomial, so in naming this species, we have used the current validly published name for the genus. The GC content of the type genome is $53.41 \%$ and the genome length is $2.2 \mathrm{Mbp}$.

\section{Description of Candidatus Ureaplasma intestinipullorum sp. nov.}

Candidatus Ureaplasma intestinipullorum (in.tes.ti.ni.pul.lo'rum. L. neut. n. intestinum gut; L. masc. n. pullus a young chicken; N.L. gen. n. intestinipullorum of the gut of young chickens)

A bacterial species identified by metagenomic analyses. This species includes all bacteria with genomes that show $\geq 95 \%$ average nucleotide identity (ANI) to the type genome, which has been assigned the MAG ID A5-1222 and which is available via NCBI BioSample SAMN15816777. The $\mathrm{GC}$ content of the type genome is $24.43 \%$ and the genome length is $0.6 \mathrm{Mbp}$.

\section{Description of Candidatus Ventrenecus gen. nov.}

Candidatus Ventrenecus (Ventren.e'cus. L. masc. n. venter the belly; Gr. masc. enoikos inhabitant; N.L. masc. n. Ventrenecus a microbe associated with faeces)

A bacterial genus identified by metagenomic analyses. The genus includes all bacteria with genomes that show $\geq 60 \%$ average amino acid identity (AAI) to the type genome from the type species Ventrenecus avicola. This is a name for the alphanumeric GTDB genus UMGS1217. This genus has been assigned by GTDB-Tk v1.3.0 working on GTDB Release 05-RS95 (Chaumeil et al., 2019; Parks et al., 2020) to the order RF39 and to the family CAG-1000.

\section{Description of Candidatus Ventrenecus avicola sp. nov.}

Candidatus Ventrenecus avicola (a.vi'co.la. L. fem. n. avis bird; L. suff. -cola inhabitant of; N.L. n. avicola inhabitant of birds)

A bacterial species identified by metagenomic analyses. This species includes all bacteria with genomes that show $\geq 95 \%$ average nucleotide identity (ANI) to the type genome, which has been assigned the MAG ID ChiW22-487 and which is available via NCBI BioSample

SAMN15817076. The GC content of the type genome is $31.09 \%$ and the genome length is 1.3 Mbp. 
9010 Description of Candidatus Ventrenecus stercoripullorum sp. nov.

9011 Candidatus Ventrenecus stercoripullorum (ster.co.ri.pul.lo'rum. L. neut. n. stercus dung; L.

9012 masc. n. pullus a young chicken; N.L. gen. n. stercoripullorum of the faceces of young chickens)

9013

9014

A bacterial species identified by metagenomic analyses. This species includes all bacteria with

9015 genomes that show $\geq 95 \%$ average nucleotide identity (ANI) to the type genome, which has been

9016 assigned the MAG ID CHK197-17881 and which is available via NCBI BioSample

9017 SAMN15817010. The GC content of the type genome is $30.90 \%$ and the genome length is 1.4

$9018 \mathrm{Mbp}$.

9019

9020

9021

Description of Candidatus Ventricola gen. nov.

9022 Candidatus Ventricola (Ven.tri.co'la. L. masc. n. venter the belly; L. suff. -cola inhabitant of;

9023 N.L. fem. n. Ventricola a microbe associated with faeces)

9024

9025

A bacterial genus identified by metagenomic analyses. The genus includes all bacteria with

9026

9027

9028 genomes that show $\geq 60 \%$ average amino acid identity (AAI) to the type genome from the type species Ventricola intestinavium. This is a name for the alphanumeric GTDB genus SFFH01.

9029 This genus has been assigned by GTDB-Tk v1.3.0 working on GTDB Release 05-RS95

9030 (Chaumeil et al., 2019; Parks et al., 2020) to the order Christensenellales and to the family $C A G$ 74.

9031

9032

9033

Description of Candidatus Ventricola gallistercoris sp. nov.

9034

Candidatus Ventricola gallistercoris (gal.li.ster'co.ris. L. masc. $\mathrm{n}$ gallus chicken; L. neut. $\mathrm{n}$.

9035

stercus dung; N.L. gen. n. gallistercoris of chicken faeces)

9036

9037

A bacterial species identified by metagenomic analyses. This species includes all bacteria with

9038

9039 genomes that show $\geq 95 \%$ average nucleotide identity (ANI) to the type genome, which has been assigned the MAG ID ChiHcec16-310 and which is available via NCBI BioSample

9040 SAMN15817100. The GC content of the type genome is $60.63 \%$ and the genome length is 2.4

9041 Mbp.

9042

9043

9044

9045

Description of Candidatus Ventricola intestinavium sp. nov.

9046

9047

9048

9049

Candidatus Ventricola intestinavium (in.tes.tin.a'vi.um. L. neut. n. intestinum gut; L. fem. n. avis bird; N.L. gen. n. intestinavium of the gut of birds)

9050

A bacterial species identified by metagenomic analyses. This species includes all bacteria with genomes that show $\geq 95 \%$ average nucleotide identity (ANI) to the type genome, which has been

9051

9052

9053

9054 assigned the MAG ID 8987 and which is available via NCBI BioSample SAMN15817130. The GC content of the type genome is $59.95 \%$ and the genome length is $2.2 \mathrm{Mbp}$.

\section{Description of Candidatus Ventrimonas gen. nov.}


9055 Candidatus Ventrimonas (Ven.tri.mo'nas. L. masc. n. venter the belly; L. fem. n. monas a

9056

9057

9058

9059

9060

9061

9062

9063

9064

9065

9066

9067

9068

9069

9070

9071

9072

9073

9074

9075

9076

9077

9078

9079

9080

9081

9082

9083

9084

9085

9086

9087

9088

9089

9090

9091

9092

9093

9094

9095

9096

9097

9098

9099

9100

monad; N.L. fem. n. Ventrimonas a microbe associated with faeces)

A bacterial genus identified by metagenomic analyses. The genus includes all bacteria with genomes that show $\geq 60 \%$ average amino acid identity (AAI) to the type genome from the type species Ventrimonas merdavium. This is a name for the alphanumeric GTDB genus UBA9502. This genus has been assigned by GTDB-Tk v1.3.0 working on GTDB Release 05-RS95 (Chaumeil et al., 2019; Parks et al., 2020) to the order Lachnospirales and to the family Lachnospiraceae.

\section{Description of Candidatus Ventrimonas merdavium sp. nov.}

Candidatus Ventrimonas merdavium (merd.a'vi.um. L. fem. n. merda faeces; L. fem. n. avis bird; N.L. gen. n. merdavium of bird faeces)

A bacterial species identified by metagenomic analyses. This species includes all bacteria with genomes that show $\geq 95 \%$ average nucleotide identity (ANI) to the type genome, which has been assigned the MAG ID USAMLcec2-739 and which is available via NCBI BioSample SAMN15817118. The GC content of the type genome is $57.43 \%$ and the genome length is 3.0 Mbp.

\section{Description of Candidatus Ventrisoma gen. nov.}

Candidatus Ventrisoma (Ven.tri.so'ma. L. masc. n. venter the belly; Gr. neut. n. soma a body; N.L. neut. n. Ventrisoma a microbe associated with faeces)

A bacterial genus identified by metagenomic analyses. The genus includes all bacteria with genomes that show $\geq 60 \%$ average amino acid identity (AAI) to the type genome from the type species Ventrisoma faecale. This is a name for the alphanumeric GTDB genus UC5-1-2E3. This genus has been assigned by GTDB-Tk v1.3.0 working on GTDB Release 05-RS95 (Chaumeil et al., 2019; Parks et al., 2020) to the order Lachnospirales and to the family Lachnospiraceae.

\section{Description of Candidatus Ventrisoma faecale sp. nov. \\ Candidatus Ventrisoma faecale (fae.ca'le. L. neut. adj. faecale of faeces)}

A bacterial species identified by metagenomic analyses. This species includes all bacteria with genomes that show $\geq 95 \%$ average nucleotide identity (ANI) to the type genome, which has been assigned the MAG ID ChiHcolR19-5415 and which is available via NCBI BioSample

SAMN15817173. This is a new name for the alphanumeric GTDB species sp001304875. The $\mathrm{GC}$ content of the type genome is $55.91 \%$ and the genome length is $2.8 \mathrm{Mbp}$.

\section{Description of Candidatus Ventrousia gen. nov.}

Candidatus Ventrousia (Ventr.ou'si.a. L. masc. n. venter the belly; Gr. fem. n. ousia an essence; N.L. fem. n. Ventrousia a microbe associated with faeces) 
9101

9102

9103

9104

9105

9106

9107

9108

9109

9110

9111

9112

9113

9114

9115

9116

9117

9118

9119

9120

9121

9122

9123

9124

9125

9126

9127

9128

9129

9130

9131

9132

9133

9134

9135

9136

9137

9138

9139

9140
A bacterial genus identified by metagenomic analyses. The genus includes all bacteria with genomes that show $\geq 60 \%$ average amino acid identity (AAI) to the type genome from the type species Ventrousia excrementavium. This is a name for the alphanumeric GTDB genus SCN-5710. This genus has been assigned by GTDB-Tk v1.3.0 working on GTDB Release 05-RS95 (Chaumeil et al., 2019; Parks et al., 2020) to the order Oscillospirales and to the family Butyricicoccaceae.

\section{Description of Candidatus Ventrousia excrementavium sp. nov.}

Candidatus Ventrousia excrementavium (ex.cre.ment.a'vi.um. L. neut. n. excrementum excrement; L. fem. n. avis bird; N.L. gen. n. excrementavium of bird excrement)

A bacterial species identified by metagenomic analyses. This species includes all bacteria with genomes that show $\geq 95 \%$ average nucleotide identity (ANI) to the type genome, which has been assigned the MAG ID CHK191-8634 and which is available via NCBI BioSample SAMN15817048. The GC content of the type genome is $57.61 \%$ and the genome length is 2.1 Mbp.

\section{Description of Candidatus Yaniella excrementavium sp. nov.}

Candidatus Yaniella excrementavium (ex.cre.ment.a'vi.um. L. neut. n. excrementum excrement; L. fem. n. avis bird; N.L. gen. n. excrementavium of bird excrement)

A bacterial species identified by metagenomic analyses. This species includes all bacteria with genomes that show $\geq 95 \%$ average nucleotide identity (ANI) to the type genome, which has been assigned the MAG ID ChiHjej13B12-778 and which is available via NCBI BioSample SAMN15816702. The GC content of the type genome is $55.30 \%$ and the genome length is 2.5 Mbp.

\section{Description of Candidatus Yaniella excrementigallinarum sp. nov.}

Candidatus Yaniella excrementigallinarum (ex.cre.men.ti.gal.li.na'rum. L. neut. n. excrementum excrement; L. fem. n. gallina hen; N.L. gen. n. excrementigallinarum of hen excrement)

A bacterial species identified by metagenomic analyses. This species includes all bacteria with genomes that show $\geq 95 \%$ average nucleotide identity (ANI) to the type genome, which has been assigned the MAG ID 4905 and which is available via NCBI BioSample SAMN15816764. The $\mathrm{GC}$ content of the type genome is $53.17 \%$ and the genome length is $2.6 \mathrm{Mbp}$. 
Table 2 (on next page)

Protologues for new taxa cultured from chicken faeces 
1 Description of Acinetobacter pecorum sp. nov.

2 (pe.co'rum M.L. gen. pl. pecorum of flocks of sheep, birds etc., as this species has been isolated

3 from chickens and sheep)

4

5

6

7

8

9

10

11

12

13

14

15

16

17

18

19

20

21

22

23

24

25

26

27

28

29

30

31

32

33

34

35

36

37

38

39

40

41

42

43

44

45

46

The type strain for this bacterial species has been cultured from chicken faeces after overnight incubation on brain-heart infusion agar at $37^{\circ} \mathrm{C}$ under aerobic conditions. The species has been assigned to this genus by the software tool GTDB-Tk v1.3.0 applied to the Genome Taxonomy Database (GTDB) Release 05-RS95 (Chaumeil et al., 2019; Parks et al., 2020). Its status as a species within this genus has been confirmed by phylogenetic analysis of all available reference genomes from the genus (Figure S3).

The species includes all bacteria with genomes that show $\geq 95 \%$ average nucleotide identity (ANI) to the genome of the type strain, which is available via GenBank accession GCA_014837015.1. The GC content of the type strain is $42.9 \%$ and the genome size is $3.2 \mathrm{Mbp}$. GTDB has given this species the alphanumerical designation sp001647535 and the two other genomes assigned to this species are derived from sheep isolates (RefSeq assembly accessions GCF_001647535.1, GCF_001647575.1) (Gupta et al., 2016). The type strain has been deposited in NCTC and DSMZ, where it can be identified via the original strain reference Sa1BUA6.

\section{Description of Arthrobacter gallicola sp. nov.}

(gal.li'co.la. L. masc. n. gallus a cock; N.L. suff. -cola an inhabitant of; N.L. masc. or fem. n. gallicola an inhabitant of the chicken)

The type strain for this bacterial species has been cultured from chicken faeces after overnight incubation on Columbia blood agar at $37^{\circ} \mathrm{C}$ under aerobic conditions. The species has been assigned to this genus by the software tool GTDB-Tk v1.3.0 applied to the Genome Taxonomy Database (GTDB) Release 05-RS95 (Chaumeil et al., 2019; Parks et al., 2020). Its status as a species within this genus has been confirmed by phylogenetic analysis of all available reference genomes from the genus (Figure S3).

The species includes all bacteria with genomes that show $\geq 95 \%$ average nucleotide identity (ANI) to the genome of the type strain, which is available via GenBank accession

GCA_014836775.1. The GC content of the type strain is $65.5 \%$ and the genome size is $3.7 \mathrm{Mbp}$. Although GTDB has assigned this species to the genus it calls Arthrobacter_B, this genus designation cannot be incorporated into a well-formed binomial, so in naming this species, we have used the basonym for the genus. The type strain has been deposited in NCTC and DSMZ, where it can be identified via the original strain reference Sa2CUA1.

\section{Description of Arthrobacter pullicola sp. nov.}

(pul.li'co.la. L. masc. n. pullus a young chicken; N.L. suff. -cola an inhabitant of; N.L. masc. or fem. n. pullicola an inhabitant of young chickens)

The type strain for this bacterial species has been cultured from chicken faeces after overnight incubation on brain-heart infusion agar at $37^{\circ} \mathrm{C}$ under aerobic conditions. The species has been assigned to this genus by the software tool GTDB-Tk v1.3.0 applied to the Genome Taxonomy Database (GTDB) Release 05-RS95 (Chaumeil et al., 2019; Parks et al., 2020). Its status as a 
47 species within this genus has been confirmed by phylogenetic analysis of all available reference

48 genomes from the genus (Figure S3).

49

50

51

52

53

54

55

56

57

58

59

60

61

62

63

64

65

66

67

68

69

70

71

72

73

74

75

76

77

78

79

80

81

82

83

84

85

86

87

88

89

90

91

92

The species includes all bacteria with genomes that show $\geq 95 \%$ average nucleotide identity (ANI) to the genome of the type strain, which is available via GenBank accession GCA_014836875.1. The GC content of the type strain is $65.7 \%$ and the genome size is $3.7 \mathrm{Mbp}$. Although GTDB has assigned this species to the genus it calls Arthrobacter_B, this genus designation cannot be incorporated into a well-formed binomial, so in naming this species, we have used the basonym for the genus. The type strain has been deposited in NCTC and DSMZ, where it can be identified via the original strain reference Sa2BUA2.

\section{Description of Bacillus norwichensis sp. nov.}

(nor.wich.en'sis. N.L. masc. adj. norwichensis pertaining to English city of Norwich, where the organism was isolated)

The type strain for this bacterial species has been cultured from chicken faeces after overnight incubation on Columbia blood agar at $37^{\circ} \mathrm{C}$ under aerobic conditions. The species has been assigned to this genus by the software tool GTDB-Tk v1.3.0 applied to the Genome Taxonomy Database (GTDB) Release 05-RS95 (Chaumeil et al., 2019; Parks et al., 2020). Its status as a species within this genus has been confirmed by phylogenetic analysis of all available reference genomes from the genus (Figure S3).

The species includes all bacteria with genomes that show $\geq 95 \%$ average nucleotide identity (ANI) to the genome of the type strain, which is available via GenBank accession GCA_014836955.1. The GC content of the type strain is $40.2 \%$ and the genome size is $4.7 \mathrm{Mbp}$. Although GTDB has assigned this species to the genus it calls Bacillus_AM, this genus designation cannot be incorporated into a well-formed binomial, so in naming this species, we have used the basonym for the genus. The type strain has been deposited in NCTC and DSMZ, where it can be identified via the original strain reference Sa1BUA2

\section{Description of Brevibacterium gallinarum sp. nov.}

(gal.li.na'rum. L. pl. gen. n. gallinarum of hens)

The type strain for this bacterial species has been cultured from chicken faeces after overnight incubation on brain-heart infusion agar at $37^{\circ} \mathrm{C}$ under aerobic conditions. The species has been assigned to this genus by the software tool GTDB-Tk v1.3.0 applied to the Genome Taxonomy Database (GTDB) Release 05-RS95 (Chaumeil et al., 2019; Parks et al., 2020). Its status as a species within this genus has been confirmed by phylogenetic analysis of all available reference genomes from the genus (Figure S3).

The species includes all bacteria with genomes that show $\geq 95 \%$ average nucleotide identity (ANI) to the genome of the type strain, which is available via GenBank accession GCA_014836885.1. The GC content of the type strain is $67.0 \%$ and the genome size is $3.2 \mathrm{Mbp}$. The type strain has been deposited in NCTC and DSMZ, where it can be identified via the original strain reference Re57

Peer) reviewing PDF | (2020:12:55980:1:1:NEW 13 Jan 2021) 
93 Description of Brevundimonas guildfordensis sp. nov.

94 (guild.ford.en'sis. N.L. fem. adj. guildfordensis pertaining to English town Guildford, home to 95 the University of Surrey, where the samples were processed)

96

97

98

99

100

101

102

103

104

105

106

107

108

109

110

111

112

113

114

115

116

117

118

119

120

121

122

123

124

125

126

127

128

129

130

131

132

133

134

135

136

137

The type strain for this bacterial species has been cultured from chicken faeces after overnight incubation on Columbia blood agar at $37^{\circ} \mathrm{C}$ under aerobic conditions. The species has been assigned to this genus by the software tool GTDB-Tk v1.3.0 applied to the Genome Taxonomy Database (GTDB) Release 05-RS95 (Chaumeil et al., 2019; Parks et al., 2020). Its status as a species within this genus has been confirmed by phylogenetic analysis of all available reference genomes from the genus (Figure S3).

The species includes all bacteria with genomes that show $\geq 95 \%$ average nucleotide identity (ANI) to the genome of the type strain, which is available via GenBank accession GCA_014836405.1. The GC content of the type strain is $67.3 \%$ and the genome size is $2.9 \mathrm{Mbp}$. The type strain has been deposited in NCTC and DSMZ, where it can be identified via the original strain reference Sa3CVA3

\section{Description of Cellulomonas avistercoris sp. nov.}

(a.vi.ster'co.ris. L. fem. n. avis bird; L. neut. n. stercus dung; N.L. gen. n. avistercoris of bird faeces)

The type strain for this bacterial species has been cultured from chicken faeces after overnight incubation on Columbia blood agar at $37^{\circ} \mathrm{C}$ under aerobic conditions. The species has been assigned to this genus by the software tool GTDB-Tk v1.3.0 applied to the Genome Taxonomy Database (GTDB) Release 05-RS95 (Chaumeil et al., 2019; Parks et al., 2020). Its status as a species within this genus has been confirmed by phylogenetic analysis of all available reference genomes from the genus (Figure S3).

The species includes all bacteria with genomes that show $\geq 95 \%$ average nucleotide identity (ANI) to the genome of the type strain, which is available via GenBank accession GCA_014836445.1. The GC content of the type strain is $74.5 \%$ and the genome size is $4.2 \mathrm{Mbp}$. The type strain has been deposited in NCTC and DSMZ, where it can be identified via the original strain reference Sa3CUA2

\section{Description of Clostridium cibarium sp. nov.}

(ci.ba'ri.um. L. neut. adj. cibarium pertaining to food, as this species has been isolated from chickens and zha-chili)

The type strain for this bacterial species has been cultured from chicken faeces after overnight incubation on Columbia blood agar at $37^{\circ} \mathrm{C}$ under anaerobic conditions. The species has been assigned to this genus by the software tool GTDB-Tk v1.3.0 applied to the Genome Taxonomy Database (GTDB) Release 05-RS95 (Chaumeil et al., 2019; Parks et al., 2020). Its status as a species within this genus has been confirmed by phylogenetic analysis of all available reference genomes from the genus (Figure S3).

Peer] reviewing PDF | (2020:12:55980:1:1:NEW 13 Jan 2021) 
138 The species includes all bacteria with genomes that show $\geq 95 \%$ average nucleotide identity 139 (ANI) to the genome of the type strain, which is available via GenBank accession

140 GCA 014836335.1. The GC content of the type strain is $29.8 \%$ and the genome size is $4.3 \mathrm{Mbp}$.

141 GTDB has given this species the alphanumerical designation sp007115085. One other isolate

142 from this species (RefSeq accession GCA_007115085.1) has been cultured from zha-chili, a

143 Chinese fermented food. The type strain has been deposited in NCTC and DSMZ, where it can

144 be identified via the original strain reference Sa3CVN1.

145

146

147

148

149

150

151

152

153

154

155

156

157

\section{Description of Clostridium gallinarum sp. nov.}

(gal.li.na'rum. L. pl. gen. n. gallinarum of hens)

The type strain for this bacterial species has been cultured from chicken faeces after overnight incubation on Columbia blood agar at $37^{\circ} \mathrm{C}$ under anaerobic conditions. The species has been assigned to this genus by the software tool GTDB-Tk v1.3.0 applied to the Genome Taxonomy Database (GTDB) Release 05-RS95 (Chaumeil et al., 2019; Parks et al., 2020). Its status as a species within this genus has been confirmed by phylogenetic analysis of all available reference genomes from the genus (Figure S3).

The species includes all bacteria with genomes that show $\geq 95 \%$ average nucleotide identity (ANI) to the genome of the type strain, which is available via GenBank accession GCA_014836325.1. The GC content of the type strain is $27.2 \%$ and the genome size is $3.4 \mathrm{Mbp}$. The type strain has been deposited in NCTC and DSMZ, where it can be identified via the original strain reference Sa3CUN1.

\section{Description of Clostridium faecium sp. nov.}

(fae'ci.um N.L. gen. pl. n. faecium, of faeces, as this species has been isolated from chicken and human faeces)

The type strain for this bacterial species has been cultured from chicken faeces after overnight incubation on brain-heart infusion agar at $37^{\circ} \mathrm{C}$ under anaerobic conditions. The species has been assigned to this genus by the software tool GTDB-Tk v1.3.0 applied to the Genome Taxonomy Database (GTDB) Release 05-RS95 (Chaumeil et al., 2019; Parks et al., 2020). Its status as a species within this genus has been confirmed by phylogenetic analysis of all available reference genomes from the genus (Figure S3).

The species includes all bacteria with genomes that show $\geq 95 \%$ average nucleotide identity (ANI) to the genome of the type strain, which is available via GenBank accession GCA_014836835.1. The GC content of the type strain is $28.7 \%$ and the genome size is $3.9 \mathrm{Mbp}$. Although GTDB has assigned this species to the genus it calls Clostridium_ $J$, this genus designation cannot be incorporated into a well-formed binomial, so in naming this species, we have used the basonym for the genus. GTDB has given this species the alphanumerical designation sp900547625, which includes a gut isolate from a preterm human infant (GenBank accession GCA_900547625.1). The type strain has been deposited in NCTC and DSMZ, where it can be identified via the original strain reference N37

182 
183

184

185

186

187

188

189

190

191

192

193

194

195

196

197

198

199

200

201

202

203

204

205

206

207

208

209

210

211

212

213

214

215

216

217

218

219

220

221

222

223

224

225

226

227

228

\section{Description of Comamonas avium sp. nov.}

(a'vi.um. L. gen. pl. n. avium of birds)

The type strain for this bacterial species has been cultured from chicken faeces after overnight incubation on brain-heart infusion agar at $37^{\circ} \mathrm{C}$ under aerobic conditions. The species has been assigned to this genus by the software tool GTDB-Tk v1.3.0 applied to the Genome Taxonomy Database (GTDB) Release 05-RS95 (Chaumeil et al., 2019; Parks et al., 2020). Its status as a species within this genus has been confirmed by phylogenetic analysis of all available reference genomes from the genus (Figure S3).

The species includes all bacteria with genomes that show $\geq 95 \%$ average nucleotide identity (ANI) to the genome of the type strain, which is available via GenBank accession

GCA_014836675.1. The GC content of the type strain is $57.5 \%$ and the genome size is $3.9 \mathrm{Mbp}$. The type strain has been deposited in NCTC and DSMZ, where it can be identified via the original strain reference Sa2CVA6

\section{Description of Corynebacterium gallinarum sp. nov.} (gal.li.na'rum. L. pl. gen. n. gallinarum of hens)

The type strain for this bacterial species has been cultured from chicken faeces after overnight incubation on YCFA (yeast extract, casitone and fatty acid) agar at $37^{\circ} \mathrm{C}$ under aerobic conditions. The species has been assigned to this genus by the software tool GTDB-Tk v1.3.0 applied to the Genome Taxonomy Database (GTDB) Release 05-RS95 (Chaumeil et al., 2019; Parks et al., 2020). Its status as a species within this genus has been confirmed by phylogenetic analysis of all available reference genomes from the genus (Figure S3).

The species includes all bacteria with genomes that show $\geq 95 \%$ average nucleotide identity (ANI) to the genome of the type strain, which is available via GenBank accession GCA_014837045.1. The GC content of the type strain is $63.1 \%$ and the genome size is $3.1 \mathrm{Mbp}$. The type strain has been deposited in NCTC and DSMZ, where it can be identified via the original strain reference Sa1YVA5

\section{Description of Cytobacillus stercorigallinarum sp. nov.}

(ster.co.ri.gal.li.na'rum. L. neut. n. stercus faeces; L. fem. n. gallina a hen; N.L. gen. n. stercorigallinarum of hen faeces)

The type strain for this bacterial species has been cultured from chicken faeces after overnight incubation on brain-heart infusion agar at $37^{\circ} \mathrm{C}$ under aerobic conditions. The species has been assigned to this genus by the software tool GTDB-Tk v1.3.0 applied to the Genome Taxonomy Database (GTDB) Release 05-RS95 (Chaumeil et al., 2019; Parks et al., 2020). Its status as a species within this genus has been confirmed by phylogenetic analysis of all available reference genomes from the genus (Figure S3).

The species includes all bacteria with genomes that show $\geq 95 \%$ average nucleotide identity (ANI) to the genome of the type strain, which is available via GenBank accession GCA_014836495.1. The GC content of the type strain is $36.8 \%$ and the genome size is $4.4 \mathrm{Mbp}$. 
229 GTDB has assigned this species to the genus it calls Bacillus_AA, which cannot be incorporated 230 into a well-formed binomial. However, according to (Patel \& Gupta, 2020), the newly named 231 genus Cytobacillus encompasses other species classified by GTDB within the genus designation 232 Bacillus_AA and therefore Cytobacillus is treated as a synonym of GTDB Bacillus_AA. The type 233 strain has been deposited in NCTC and DSMZ, where it can be identified via the original strain 234 reference Sa5YUA1.

235

272

\section{Description of Escherichia whittamii sp. nov.}

\section{(whitt.am'i.i. N.L. gen. n. whittamii, named in honour of American microbiologist Thomas S.} Whittam)

The type strain for this bacterial species has been cultured from chicken faeces after overnight incubation on Columbia blood agar at $37^{\circ} \mathrm{C}$ under anaerobic conditions. The species has been assigned to this genus by the software tool GTDB-Tk v1.3.0 applied to the Genome Taxonomy Database (GTDB) Release 05-RS95 (Chaumeil et al., 2019; Parks et al., 2020). Its status as a species within this genus has been confirmed by phylogenetic analysis of reference genomes from the genus (Figure 4).

The species includes all bacteria with genomes that show $\geq 95 \%$ average nucleotide identity (ANI) to the genome of the type strain, which is available via GenBank accession

GCA_014836715.1. The GC content of the type strain is 50.6\% and the genome size is $4.6 \mathrm{Mbp}$. GTD $\bar{B}$ has given this species the alphanumerical designation sp001660175 and has assigned two other cultured isolates to this species, both of which come from birds (RefSeq assembly accessions GCF_001660175.1, GCF_002965485.1) (Clermont et al., 2011; Gangiredla et al., 2018) The type strain has been deposited in NCTC and DSMZ, where it can be identified via the original strain reference Sa2BVA5.

\section{Description of Fictibacillus norfolkensis sp. nov.}

(nor.folk.en_sis. N.L. masc. adj. norfolkensis pertaining to the English county of Norfolk)

The type strain for this bacterial species has been cultured from chicken faeces after overnight incubation on YCFA (yeast extract, casitone and fatty acid) agar at $37^{\circ} \mathrm{C}$ under aerobic conditions. The species has been assigned to this genus by the software tool GTDB-Tk v1.3.0 applied to the Genome Taxonomy Database (GTDB) Release 05-RS95 (Chaumeil et al., 2019; Parks et al., 2020). Its status as a species within this genus has been confirmed by phylogenetic analysis of all available reference genomes from the genus (Figure S3).

The species includes all bacteria with genomes that show $\geq 95 \%$ average nucleotide identity (ANI) to the genome of the type strain, which is available via GenBank accession GCA_014836645.1. The GC content of the type strain is 39.5\% and the genome size is $4.0 \mathrm{Mbp}$. Although GTDB has assigned this species to the genus it calls Fictibacillus_B, this genus designation cannot be incorporated into a well-formed binomial, so in naming this species, we have used the basonym for the genus. The type strain has been deposited in NCTC and DSMZ, where it can be identified via the original strain reference Sa2CUA10 
274 Description of Kaistella pullorum sp. nov.

275 (pul.lor_um. L. gen. pl. n. pullorum of chickens)

276

277

278

279

280

281

282

283

284

285

286

287

288

289

290

291

292

293

294

295

296

297

298

299

300

301

302

303

304

305

306

307

308

309

310

311

312

313

314

315

316

317

318

The type strain for this bacterial species has been cultured from chicken faeces after overnight incubation on Columbia blood agar at $37^{\circ} \mathrm{C}$ under aerobic conditions. The species has been assigned to this genus by the software tool GTDB-Tk v1.3.0 applied to the Genome Taxonomy Database (GTDB) Release 05-RS95 (Chaumeil et al., 2019; Parks et al., 2020). Its status as a species within this genus has been confirmed by phylogenetic analysis of all available reference genomes from the genus (Figure S3).

The species includes all bacteria with genomes that show $\geq 95 \%$ average nucleotide identity (ANI) to the genome of the type strain, which is available via GenBank accession

GCA_014837035.1. The GC content of the type strain is $42.9 \%$ and the genome size is $2.6 \mathrm{Mbp}$.

The type strain has been deposited in NCTC and DSMZ, where it can be identified via the original strain reference Sa1CVA4

\section{Description of Limosilactobacillus avistercoris sp. nov.}

(a.vi.ster'co.ris. L. fem. n. avis bird; L. neut. n. stercus dung; N.L. gen. n. avistercoris of bird faeces)

The type strain for this bacterial species has been cultured from chicken faeces after overnight incubation on brain-heart infusion agar at $37^{\circ} \mathrm{C}$ under anaerobic conditions. The species has been assigned to this genus by the software tool GTDB-Tk v1.3.0 applied to the Genome Taxonomy Database (GTDB) Release 05-RS95 (Chaumeil et al., 2019; Parks et al., 2020). Its status as a species within this genus has been confirmed by phylogenetic analysis of all available reference genomes from the genus (Figure S3).

The species includes all bacteria with genomes that show $\geq 95 \%$ average nucleotide identity (ANI) to the genome of the type strain, which is available via GenBank accession GCA_014836425.1. The GC content of the type strain is $39.9 \%$ and the genome size is $1.8 \mathrm{Mbp}$. GTDB has assigned this species to the genus it calls Lactobacillus_H, which cannot be incorporated into a well-formed binomial. However, according to (Zheng et al., 2020) Limosilactobacillus encompasses other species classified by GTDB within the genus designation Lactobacillus_H and therefore Limosilactobacillus is treated as a synonym for GDTB designation Lactobacillus_H. The type strain has been deposited in NCTC and DSMZ, where it can be identified via the original strain reference Sa3CUN2

\section{Description of Luteimonas colneyensis sp. nov.}

(col.ney.en'sis. N.L. fem. adj. colneyensis pertaining to the English village of Colney, home to the Quadram Institute, where the species was first described)

The type strain for this bacterial species has been cultured from chicken faeces after overnight incubation on Columbia blood agar at $37^{\circ} \mathrm{C}$ under aerobic conditions. The species has been assigned to this genus by the software tool GTDB-Tk v1.3.0 applied to the Genome Taxonomy Database (GTDB) Release 05-RS95 (Chaumeil et al., 2019; Parks et al., 2020). Its status as a 
species within this genus has been confirmed by phylogenetic analysis of all available reference genomes from the genus (Figure S3).

The species includes all bacteria with genomes that show $\geq 95 \%$ average nucleotide identity (ANI) to the genome of the type strain, which is available via GenBank accession GCA_014836665.1. The GC content of the type strain is $71.0 \%$ and the genome size is $3.0 \mathrm{Mbp}$. The type strain has been deposited in NCTC and DSMZ, where it can be identified via the original strain reference Sa2BVA3

\section{Description of Microbacterium commune sp. nov.} (com.mu_ne. L. neut. adj. commune common, referring to diverse habitats, as this species has been isolated from mosquitos and chicken)

The type strain for this bacterial species has been cultured from chicken faeces after overnight incubation on Columbia blood agar at $37^{\circ} \mathrm{C}$ under aerobic conditions. The species has been assigned to this genus by the software tool GTDB-Tk v1.3.0 applied to the Genome Taxonomy Database (GTDB) Release 05-RS95 (Chaumeil et al., 2019; Parks et al., 2020). Its status as a species within this genus has been confirmed by phylogenetic analysis of all available reference genomes from the genus (Figure S3).

The species includes all bacteria with genomes that show $\geq 95 \%$ average nucleotide identity (ANI) to the genome of the type strain, which is available via GenBank accession GCA_014836945.1. The GC content of the type strain is $70.3 \%$ and the genome size is $3.3 \mathrm{Mbp}$. GTDB has given this species the alphanumerical designation sp001878835, which also contains a mosquito isolate (RefSeq accession GCF_001878835.1). The type strain has been deposited in NCTC and DSMZ, where it can be identified via the original strain reference Re1.

\section{Description of Microbacterium gallinarum sp. nov.}

(gal.li.na'rum. L. pl. gen. n. gallinarum of hens)

The type strain for this bacterial species has been cultured from chicken faeces after overnight incubation on brain-heart infusion agar at $37^{\circ} \mathrm{C}$ under aerobic conditions. The species has been assigned to this genus by the software tool GTDB-Tk v1.3.0 applied to the Genome Taxonomy Database (GTDB) Release 05-RS95 (Chaumeil et al., 2019; Parks et al., 2020). Its status as a species within this genus has been confirmed by phylogenetic analysis of all available reference genomes from the genus (Figure S3).

The species includes all bacteria with genomes that show $\geq 95 \%$ average nucleotide identity (ANI) to the genome of the type strain, which is available via GenBank accession GCA_014837165.1. The GC content of the type strain is $69.4 \%$ and the genome size is $2.8 \mathrm{Mbp}$. The type strain has been deposited in NCTC and DSMZ, where it can be identified via the original strain reference Sa1CUA4

\section{Description of Microbacterium pullorum sp. nov.} (pul.lor_um. L.gen. pl.n. pullorum of chickens) 
364

365

366

367

368

369

370

371

372

373

374

375

376

377

378

379

380

381

382

383

384

385

386

387

388

389

390

391

392

393

394

395

396

397

398

399

400

401

402

403

404

405

406

407

408

409

The type strain for this bacterial species has been cultured from chicken faeces after overnight incubation on Columbia blood agar at $37^{\circ} \mathrm{C}$ under aerobic conditions. The species has been assigned to this genus by the software tool GTDB-Tk v1.3.0 applied to the Genome Taxonomy Database (GTDB) Release 05-RS95 (Chaumeil et al., 2019; Parks et al., 2020). Its status as a species within this genus has been confirmed by phylogenetic analysis of all available reference genomes from the genus (Figure S3).

The species includes all bacteria with genomes that show $\geq 95 \%$ average nucleotide identity (ANI) to the genome of the type strain, which is available via GenBank accession GCA_014836535.1. The GC content of the type strain is $70.1 \%$ and the genome size is $3.1 \mathrm{Mbp}$. The type strain has been deposited in NCTC and DSMZ, where it can be identified via the original strain reference Sa4CUA7

\section{Description of Oceanitalea stevensii sp. nov.}

(ste.ven'si.i. N.L. gen. n. stevensii, named in honour of British microbiologist Mark Stevens)

The type strain for this bacterial species has been cultured from chicken faeces after overnight incubation on brain-heart infusion agar at $37^{\circ} \mathrm{C}$ under aerobic conditions. The species has been assigned to this genus by the software tool GTDB-Tk v1.3.0 applied to the Genome Taxonomy Database (GTDB) Release 05-RS95 (Chaumeil et al., 2019; Parks et al., 2020). Its status as a species within this genus has been confirmed by phylogenetic analysis of all available reference genomes from the genus (Figure S3).

The species includes all bacteria with genomes that show $\geq 95 \%$ average nucleotide identity (ANI) to the genome of the type strain, which is available via GenBank accession GCA_014837105.1. The GC content of the type strain is $73.4 \%$ and the genome size is $3.5 \mathrm{Mbp}$. The type strain has been deposited in NCTC and DSMZ, where it can be identified via the original strain reference Sa1BUA1.

\section{Description of Ochrobactrum gallinarum sp. nov. (gal.li.na'rum. L. pl. gen. n. gallinarum of hens)}

The type strain for this bacterial species has been cultured from chicken faeces after overnight incubation on brain-heart infusion at $37^{\circ} \mathrm{C}$ under aerobic conditions. The species has been assigned to this genus by the software tool GTDB-Tk v1.3.0 applied to the Genome Taxonomy Database (GTDB) Release 05-RS95 (Chaumeil et al., 2019; Parks et al., 2020). Its status as a species within this genus has been confirmed by phylogenetic analysis of all available reference genomes from the genus (Figure S3).

The species includes all bacteria with genomes that show $\geq 95 \%$ average nucleotide identity (ANI) to the genome of the type strain, which is available via GenBank accession GCA_014836735.1. The GC content of the type strain is $53.5 \%$ and the genome size is $5.0 \mathrm{Mbp}$. The type strain has been deposited in NCTC and DSMZ, where it can be identified via the original strain reference Sa2BUA5

Peer) reviewing PDF | (2020:12:55980:1:1:NEW 13 Jan 2021) 
410 Description of Oerskovia douganii sp. nov.

411 (dou.gan_i.i. N.L. gen. n. douganii named in honour of British microbiologist Gordon Dougan)

412

413

414

415

416

417

418

419

420

421

422

423

424

425

426

427

428

429

430

431

432

433

434

435

436

437

438

439

440

441

442

443

444

445

446

447

448

449

450

451

452

453

454

455

The type strain for this bacterial species has been cultured from chicken faeces after overnight incubation on brain-heart infusion at $37^{\circ} \mathrm{C}$ under aerobic conditions. The species has been assigned to this genus by the software tool GTDB-Tk v1.3.0 applied to the Genome Taxonomy Database (GTDB) Release 05-RS95 (Chaumeil et al., 2019; Parks et al., 2020). Its status as a species within this genus has been confirmed by phylogenetic analysis of all available reference genomes from the genus (Figure S3).

The species includes all bacteria with genomes that show $\geq 95 \%$ average nucleotide identity (ANI) to the genome of the type strain, which is available via GenBank accession

GCA_015142735.1. The GC content of the type strain is $72.5 \%$ and the genome size is $4.3 \mathrm{Mbp}$. The type strain has been deposited in NCTC and DSMZ, where it can be identified via the original strain reference Sa1BUA8

\section{Description of Oerskovia gallyi sp. nov.}

(gall.y'i. N.L. gen. n. gallyi named in honour of British microbiologist David Gally)

The type strain for this bacterial species has been cultured from chicken faeces after overnight incubation on brain-heart infusion agar at $37^{\circ} \mathrm{C}$ under aerobic conditions. The species has been assigned to this genus by the software tool GTDB-Tk v1.3.0 applied to the Genome Taxonomy Database (GTDB) Release 05-RS95 (Chaumeil et al., 2019; Parks et al., 2020). Its status as a species within this genus has been confirmed by phylogenetic analysis of all available reference genomes from the genus (Figure S3).

The species includes all bacteria with genomes that show $\geq 95 \%$ average nucleotide identity (ANI) to the genome of the type strain, which is available via GenBank accession GCA_014836745.1. The GC content of the type strain is $72.5 \%$ and the genome size is $4.3 \mathrm{Mbp}$. The type strain has been deposited in NCTC and DSMZ, where it can be identified via the original strain reference Sa2CUA8

\section{Description of Oerskovia merdavium sp. nov.}

(merd.a'vi.um. L. fem. n. merda faeces; L. fem. n. avis bird; N.L. gen. n. merdavium of bird faeces)

The type strain for this bacterial species has been cultured from chicken faeces after overnight incubation on Columbia blood agar at $37^{\circ} \mathrm{C}$ under aerobic conditions. The species has been assigned to this genus by the software tool GTDB-Tk v1.3.0 applied to the Genome Taxonomy Database (GTDB) Release 05-RS95 (Chaumeil et al., 2019; Parks et al., 2020). Its status as a species within this genus has been confirmed by phylogenetic analysis of all available reference genomes from the genus (Figure S3).

The species includes all bacteria with genomes that show $\geq 95 \%$ average nucleotide identity (ANI) to the genome of the type strain, which is available via GenBank accession GCA_014836755.1. The GC content of the type strain is $72.1 \%$ and the genome size is $4.5 \mathrm{Mbp}$.

Peer) reviewing PDF | (2020:12:55980:1:1:NEW 13 Jan 2021) 
456 The type strain has been deposited in NCTC and DSMZ, where it can be identified via the 457 original strain reference Sa2CUA9.

458

459

460

461

462

463

464

465

466

467

468

469

470

471

472

473

474

475

476

477

478

479

480

481

482

483

484

485

486

487

488

489

490

491

492

493

494

495

496

497

498

499

\section{Description of Oerskovia rustica sp. nov.}

(rus'tic.a L fem adj. rustica of the countryside, as isolates have been obtained from soil and chickens)

The type strain for this bacterial species has been cultured from chicken faeces after overnight incubation on Columbia blood agar at $37^{\circ} \mathrm{C}$ under aerobic conditions. The species has been assigned to this genus by the software tool GTDB-Tk v1.3.0 applied to the Genome Taxonomy Database (GTDB) Release 05-RS95 (Chaumeil et al., 2019; Parks et al., 2020). Its status as a species within this genus has been confirmed by phylogenetic analysis of all available reference genomes from the genus (Figure S3).

The species includes all bacteria with genomes that show $\geq 95 \%$ average nucleotide identity (ANI) to the genome of the type strain, which is available via GenBank accession GCA_014836555.1. The GC content of the type strain is $72.5 \%$ and the genome size is $4.4 \mathrm{Mbp}$. GTDB has given this species the alphanumerical designation sp005937995, which includes a soil isolate (RefSeq accession GCF_005937995.2). The type strain has been deposited in NCTC and DSMZ, where it can be identified via the original strain reference Sa4CUA1.

\section{Description of Paenibacillus gallinarum sp. nov.}

(gal.li.na'rum. L. pl. gen. n. gallinarum of hens)

The type strain for this bacterial species has been cultured from chicken faeces after overnight incubation on Columbia blood agar at $37^{\circ} \mathrm{C}$ under aerobic conditions. The species has been assigned to this genus by the software tool GTDB-Tk v1.3.0 applied to the Genome Taxonomy Database (GTDB) Release 05-RS95 (Chaumeil et al., 2019; Parks et al., 2020). Its status as a species within this genus has been confirmed by phylogenetic analysis of all available reference genomes from the genus (Figure S3).

The species includes all bacteria with genomes that show $\geq 95 \%$ average nucleotide identity (ANI) to the genome of the type strain, which is available via GenBank accession GCA_014836635.1. The GC content of the type strain is $41.2 \%$ and the genome size is $5.4 \mathrm{Mbp}$. The type strain has been deposited in NCTC and DSMZ, where it can be identified via the original strain reference Sa2BVA9

\section{Description of Phocaeicola faecium sp. nov.}

(fae'ci.um N.L. gen. pl. n. faecium, of faeces)

The type strain for this bacterial species has been cultured from chicken faeces after overnight incubation on YCFA (yeast extract, casitone and fatty acid) agar at $37^{\circ} \mathrm{C}$ under anaerobic conditions. The species has been assigned to this genus by the software tool GTDB-Tk v1.3.0 applied to the Genome Taxonomy Database (GTDB) Release 05-RS95 (Chaumeil et al., 2019; 
500 Parks et al., 2020). Its status as a species within this genus has been confirmed by phylogenetic

501

502

503

504

505

506

507

508

509

510

511

512

513

514

515

516

517

518

519

520

521

522

523

524

525

526

527

528

529

530

531

532

533

534

535

536

537

538

539

540

541

542

543

544

analysis of all available reference genomes from the genus (Figure S3).

The species includes all bacteria with genomes that show $\geq 95 \%$ average nucleotide identity (ANI) to the genome of the type strain, which is available via GenBank accession

GCA_014837055.1. The GC content of the type strain is $45.6 \%$ and the genome size is $3.5 \mathrm{Mbp}$. GTDB has given this species the alphanumerical designation sp900540105, which includes a gut isolate from an infant human (GenBank accession GCA_900540105.1). The type strain has been deposited in NCTC and DSMZ, where it can be identified via the original strain reference Sa1YUN3

\section{Description of Phocaeicola intestinalis sp. nov.}

(in.tes.ti.na'lis. N.L. masc./fem. adj. intestinalis, pertaining to the intestines)

The type strain for this bacterial species has been cultured from chicken faeces after overnight incubation on Columbia blood agar at $37^{\circ} \mathrm{C}$ under anaerobic conditions. The species has been assigned to this genus by the software tool GTDB-Tk v1.3.0 applied to the Genome Taxonomy Database (GTDB) Release 05-RS95 (Chaumeil et al., 2019; Parks et al., 2020). Its status as a species within this genus has been confirmed by phylogenetic analysis of all available reference genomes from the genus (Figure S3).

The species includes all bacteria with genomes that show $\geq 95 \%$ average nucleotide identity (ANI) to the genome of the type strain, which is available via GenBank accession GCA_014837065.1. The GC content of the type strain is $45.7 \%$ and the genome size is $4.1 \mathrm{Mbp}$. GTDB has given this species the alphanumerical designation sp002161565, which includes isolates from the human and chicken guts (GenBank accession numbers GCA_000432695.1, GCA_900540165.1, GCF_002159615.1, GCF_002159755.1, GCF_002160215.1, GCF_002161565.1). The type strain has been deposited in NCTC and DSMZ, where it can be identified via the original strain reference Sa1CVN1.

\section{Description of Planococcus wigleyi sp. nov.}

(wig_ley.i. N.L. masc. gen. n. wigleyi named in honour of British microbiologist Paul Wigley)

The type strain for this bacterial species has been cultured from chicken faeces after overnight incubation on brain-heart infusion agar at $37^{\circ} \mathrm{C}$ under aerobic conditions. The species has been assigned to this genus by the software tool GTDB-Tk v1.3.0 applied to the Genome Taxonomy Database (GTDB) Release 05-RS95 (Chaumeil et al., 2019; Parks et al., 2020). Its status as a species within this genus has been confirmed by phylogenetic analysis of all available reference genomes from the genus (Figure S3).

The species includes all bacteria with genomes that show $\geq 95 \%$ average nucleotide identity (ANI) to the genome of the type strain, which is available via GenBank accession GCA_014836985.1. The GC content of the type strain is $45.0 \%$ and the genome size is $3.8 \mathrm{Mbp}$. Although GTDB has assigned a genus name with an alphabetic suffix Planococcus_A, this genus designation cannot be incorporated into a well-formed binomial, so in naming this species, we

Peer] reviewing PDF | (2020:12:55980:1:1:NEW 13 Jan 2021) 
545 have used the basonym for the genus. The type strain has been deposited in NCTC and DSMZ,

546 where it can be identified via the original strain reference Sa1BUA13

547

548 Description of Psychrobacillus faecigallinarum sp. nov.

549 (fae.ci.gal.li.na'rum. L. fem. n. faex, faecis faeces; L. fem. n. gallina a hen; N.L. gen. n.

550 faecigallinarum of hen faeces)

551

552

553

554

555

556

557

558

559

560

561

562

563

564

565

566

567

568

569

570

571

572

573

574

575

576

577

578

579

580

581

582

583

584

585

586

587

588

589

The type strain for this bacterial species has been cultured from chicken faeces after overnight incubation on brain-heart infusion agar at $37^{\circ} \mathrm{C}$ under aerobic conditions. The species has been assigned to this genus by the software tool GTDB-Tk v1.3.0 applied to the Genome Taxonomy Database (GTDB) Release 05-RS95 (Chaumeil et al., 2019; Parks et al., 2020). Its status as a species within this genus has been confirmed by phylogenetic analysis of all available reference genomes from the genus (Figure S3).

The species includes all bacteria with genomes that show $\geq 95 \%$ average nucleotide identity (ANI) to the genome of the type strain, which is available via GenBank accession GCA_014836595.1. The GC content of the type strain is $36.5 \%$ and the genome size is $4.0 \mathrm{Mbp}$. The type strain has been deposited in NCTC and DSMZ, where it can be identified via the original strain reference Sa2BUA9

\section{Description of Psychrobacter communis sp. nov.}

(com.mun is L. masc. adj. communis common, referring to diverse habitats from which this species has been isolated, including chickens and soil)

The type strain for this bacterial species has been cultured from chicken faeces after overnight incubation on xyz medium at $37^{\circ} \mathrm{C}$ under aerobic conditions. The species has been assigned to this genus by the software tool GTDB-Tk v1.3.0 applied to the Genome Taxonomy Database (GTDB) Release 05-RS95 (Chaumeil et al., 2019; Parks et al., 2020). Its status as a species within this genus has been confirmed by phylogenetic analysis of all available reference genomes from the genus (Figure S3).

The species includes all bacteria with genomes that show $\geq 95 \%$ average nucleotide identity (ANI) to the genome of the type strain, which is available via GenBank accession GCA_014836505.1. The GC content of the type strain is $43.7 \%$ and the genome size is $3.0 \mathrm{Mbp}$. GTDB has given this species the alphanumerical designation sp001652315, which contains six environmental isolates from a variety of sources including soil (GCA_002332465.1, GCA_002439405.1, GCA_003524605.1, GCA_007280595.1, GCF_001652315.1, GCF_002836335.1.) The type strain has been deposited in NCTC and DSMZ, where it can be identified via the original strain reference Sa4CVA2

\section{Description of Serpens gallinarum sp. nov.} (gal.li.na'rum. L. pl. gen. n. gallinarum of hens)

The type strain for this bacterial species has been cultured from chicken faeces after overnight incubation on Columbia blood agar at $37^{\circ} \mathrm{C}$ under aerobic conditions. The species has been

Peer) reviewing PDF | (2020:12:55980:1:1:NEW 13 Jan 2021) 
590

591

592

593

594

595

596

597

598

599

600

601

602

603

604

605

606

607

608

609

610

611

612

613

614

615

616

617

618

619

620

621

622

623

624

625

626

627

628

629

630

631

632

633

634

assigned to this genus by the software tool GTDB-Tk v1.3.0 applied to the Genome Taxonomy Database (GTDB) Release 05-RS95 (Chaumeil et al., 2019; Parks et al., 2020). Its status as a species within this genus has been confirmed by phylogenetic analysis of all available reference genomes from the genus (Figure S3).

The species includes all bacteria with genomes that show $\geq 95 \%$ average nucleotide identity (ANI) to the genome of the type strain, which is available via GenBank accession GCA_014836765.1. The GC content of the type strain is $61.0 \%$ and the genome size is $3.9 \mathrm{Mbp}$. Although GTDB has assigned a genus name with an alphabetic suffix Pseudomonas_H, which cannot be incorporated into a well-formed binomial. However, GDTB genus Pseudomonas_H includes Pseudomonas flexibilis, where the basonym is Serpens (Hespell, 1977), so we have used this genus name. The type strain has been deposited in NCTC and DSMZ, where it can be identified via the original strain reference Sa2CUA2

\section{Description of Solibacillus faecavium sp. nov.}

(faec.a'vi.um. L. fem. n. faex, faecis faeces; L. fem. n. avis bird; N.L. gen. n. faecavium of bird faeces)

The type strain for this bacterial species has been cultured from chicken faeces after overnight incubation on brain-heart infusion agar at $37^{\circ} \mathrm{C}$ under aerobic conditions. The species has been assigned to this genus by the software tool GTDB-Tk v1.3.0 applied to the Genome Taxonomy Database (GTDB) Release 05-RS95 (Chaumeil et al., 2019; Parks et al., 2020). Its status as a species within this genus has been confirmed by phylogenetic analysis of all available reference genomes from the genus (Figure S3).

The species includes all bacteria with genomes that show $\geq 95 \%$ average nucleotide identity (ANI) to the genome of the type strain, which is available via GenBank accession

GCA_014836905.1. The GC content of the type strain is $37.1 \%$ and the genome size is $3.8 \mathrm{Mbp}$. The type strain has been deposited in NCTC and DSMZ, where it can be identified via the original strain reference A46

\section{Description of Solibacillus merdavium sp. nov.}

(merd.a'vi.um. L. fem. n. merda faeces; L. fem. n. avis bird; N.L. gen. n. merdavium of bird faeces)

The type strain for this bacterial species has been cultured from chicken faeces after overnight incubation on YCFA (yeast extract, casitone and fatty acid) agar at $37^{\circ} \mathrm{C}$ under aerobic conditions. The species has been assigned to this genus by the software tool GTDB-Tk v1.3.0 applied to the Genome Taxonomy Database (GTDB) Release 05-RS95 (Chaumeil et al., 2019; Parks et al., 2020). Its status as a species within this genus has been confirmed by phylogenetic analysis of all available reference genomes from the genus (Figure S3).

The species includes all bacteria with genomes that show $\geq 95 \%$ average nucleotide identity (ANI) to the genome of the type strain, which is available via GenBank accession GCA_014836935.1. The GC content of the type strain is $37.0 \%$ and the genome size is $3.8 \mathrm{Mbp}$.

Peer) reviewing PDF | (2020:12:55980:1:1:NEW 13 Jan 2021) 
635 The type strain has been deposited in NCTC and DSMZ, where it can be identified via the 636 original strain reference Sa1YVA6

637

\section{Description of Sporosarcina gallistercoris sp. nov.}

640

641

642

643 (gal.li.ster'co.ris. L. masc. n. gallus a cock; L. neut. n. stercus dung; N.L. gen. n. gallistercoris of faeces of a cock)

644

645

The type strain for this bacterial species has been cultured from chicken faeces after overnight incubation on YCFA (yeast extract, casitone and fatty acid) agar at $37^{\circ} \mathrm{C}$ under aerobic conditions. The species has been assigned to this genus by the software tool GTDB-Tk v1.3.0 applied to the Genome Taxonomy Database (GTDB) Release 05-RS95 (Chaumeil et al., 2019;

646

647

648

649

650 Parks et al., 2020). Its status as a species within this genus has been confirmed by phylogenetic analysis of all available reference genomes from the genus (Figure S3).

The species includes all bacteria with genomes that show $\geq 95 \%$ average nucleotide identity (ANI) to the genome of the type strain, which is available via GenBank accession GCA_014836415.1. The GC content of the type strain is $44.1 \%$ and the genome size is $3.1 \mathrm{Mbp}$. Although GTDB has assigned a genus name with an alphabetic suffix Sporosarcina_A, this genus designation cannot be incorporated into a well-formed binomial, so in naming this species, we have used the basonym for the genus. The type strain has been deposited in NCTC and DSMZ, where it can be identified via the original strain reference Sa3CUA8

656

\section{Description of Sporosarcina quadrami sp. nov.}

(qua.dra'mi. N.L. gen. n. quadrami, of the Quadram Institute, where the species was first cultured.)

The type strain for this bacterial species has been cultured from chicken faeces after overnight incubation on brain-heart infusion agar at $37^{\circ} \mathrm{C}$ under aerobic conditions. The species has been assigned to this genus by the software tool GTDB-Tk v1.3.0 applied to the Genome Taxonomy Database (GTDB) Release 05-RS95 (Chaumeil et al., 2019; Parks et al., 2020). Its status as a species within this genus has been confirmed by phylogenetic analysis of all available reference genomes from the genus (Figure S3).

The species includes all bacteria with genomes that show $\geq 95 \%$ average nucleotide identity (ANI) to the genome of the type strain, which is available via GenBank accession GCA_014836615.1. The GC content of the type strain is $41.4 \%$ and the genome size is $3.6 \mathrm{Mbp}$. Although GTDB has assigned a genus name with an alphabetic suffix Sporosarcina_B, this genus designation cannot be incorporated into a well-formed binomial, so in naming this species, we have used the basonym for the genus. The type strain has been deposited in NCTC and

675

676

677 DSMZ, where it can be identified via the original strain reference Sa2YVA2

678

\section{Description of Stenotrophomonas pennii sp. nov.} (pen'ni.i. N.L. gen. n. pennii, named in honour of British microbiologist Charles W. Penn) 
679 The type strain for this bacterial species has been cultured from chicken faeces after overnight 680 incubation on brain-heart infusion agar at $37^{\circ} \mathrm{C}$ under aerobic conditions. The species has been 681 assigned to this genus by the software tool GTDB-Tk v1.3.0 applied to the Genome Taxonomy 682 Database (GTDB) Release 05-RS95 (Chaumeil et al., 2019; Parks et al., 2020). Its status as a 683 species within this genus has been confirmed by phylogenetic analysis of all available reference 684 genomes from the genus (Figure S3).

685

686

687 The species includes all bacteria with genomes that show $\geq 95 \%$ average nucleotide identity (ANI) to the genome of the type strain, which is available via GenBank accession

688 GCA_014836545.1. The GC content of the type strain is $66.4 \%$ and the genome size is $3.9 \mathrm{Mbp}$. 689 GTDB has given this species the alphanumerical designation sp002836635, which includes four 690 environmental isolates (RefSeq accessions GCF_000834105.1, GCF_002836635.1, 691 GCF_002836645.1, GCF_002836675.1). The type strain has been deposited in NCTC and 692 DSMZ, where it can be identified via the original strain reference Sa5BUN4

693

694

695

696

697

\section{Description of Ureibacillus galli sp. nov.}

(gal_li. L. masc. gen. n. galli of a chicken)

700

701

702

703

704

705

706

707

708

709

710

711

712

713

714

715

716

717

718

719

720

721 The species includes all bacteria with genomes that show $\geq 95 \%$ average nucleotide identity

722

723

724

The type strain for this bacterial species has been cultured from chicken faeces after overnight incubation on brain-heart infusion agar at $37^{\circ} \mathrm{C}$ under aerobic conditions. The species has been assigned to this genus by the software tool GTDB-Tk v1.3.0 applied to the Genome Taxonomy Database (GTDB) Release 05-RS95 (Chaumeil et al., 2019; Parks et al., 2020). Its status as a species within this genus has been confirmed by phylogenetic analysis of all available reference genomes from the genus (Figure S3).

The species includes all bacteria with genomes that show $\geq 95 \%$ average nucleotide identity (ANI) to the genome of the type strain, which is available via GenBank accession GCA_014836845.1. The GC content of the type strain is $35.2 \%$ and the genome size is $3.7 \mathrm{Mbp}$. The type strain has been deposited in NCTC and DSMZ, where it can be identified via the original strain reference Re31.

\section{Description of Xanthomonas surreyensis sp. nov.}

(sur.rey.en'sis. N.L. fem. adj. surreyensis pertaining to the English county of Surrey, where the samples were obtained)

The type strain for this bacterial species has been cultured from chicken faeces after overnight incubation on brain-heart infusion agar at $37^{\circ} \mathrm{C}$ under aerobic conditions. The species has been assigned to this genus by the software tool GTDB-Tk v1.3.0 applied to the Genome Taxonomy Database (GTDB) Release 05-RS95 (Chaumeil et al., 2019; Parks et al., 2020). Its status as a species within this genus has been confirmed by phylogenetic analysis of all available reference genomes from the genus (Figure S3). (ANI) to the genome of the type strain, which is available via GenBank accession GCA_014836395.1. The GC content of the type strain is $68.8 \%$ and the genome size is $5.4 \mathrm{Mbp}$. Although GTDB has assigned a genus name with an alphabetic suffix Xanthomonas_A, this 
725

726

727

728

729

730

731

732

733

734

735

736

737

738

739

740

741

742

743

744

745

746

747

748

749

750

751

752

753

754

755

756

757

758

759

760

761

762

genus designation cannot be incorporated into a well-formed binomial, so in naming this species, we have used the basonym for the genus. The type strain has been deposited in NCTC and DSMZ, where it can be identified via the original strain reference Sa3BUA13.

\section{References}

Chaumeil, P. A., Mussig, A. J., Hugenholtz, P., \& Parks, D. H. (2019). GTDB-Tk: a toolkit to classify genomes with the Genome Taxonomy Database. Bioinformatics. https://doi.org/10.1093/bioinformatics/btz848

Clermont, O., Gordon, D. M., Brisse, S., Walk, S. T., \& Denamur, E. (2011). Characterization of the cryptic Escherichia lineages: rapid identification and prevalence. Environ Microbiol, 13(9), 2468-2477. https://doi.org/10.1111/j.1462-2920.2011.02519.x

Gangiredla, J., Mammel, M. K., Barnaba, T. J., Tartera, C., Gebru, S. T., Patel, I. R., Leonard, S. R., Kotewicz, M. L., Lampel, K. A., Elkins, C. A., \& Lacher, D. W. (2018). Draft Genome Sequences of Escherichia albertii, Escherichia fergusonii, and Strains Belonging to Six Cryptic Lineages of Escherichia spp. Genome Announc, 6(18). https://doi.org/10.1128/genomeA.00271-18

Gupta, V., Haider, S., Sood, U., Gilbert, J. A., Ramjee, M., Forbes, K., Singh, Y., Lopes, B. S., \& Lal, R. (2016). Comparative genomic analysis of novel Acinetobacter symbionts: A combined systems biology and genomics approach. Sci Rep, 6, 29043. https://doi.org/10.1038/srep29043

Hespell, R. B. (1977). Serpens flexibilis gen. nov., sp. nov., an Unusually Flexible, LactateOxidizing Bacterium. International Journal of Systematic Bacteriology, 27(4), 371-381. https://doi.org/10.1099/00207713-27-4-371

Parks, D. H., Chuvochina, M., Chaumeil, P. A., Rinke, C., Mussig, A. J., \& Hugenholtz, P. (2020). A complete domain-to-species taxonomy for Bacteria and Archaea. Nat Biotechnol. https://doi.org/10.1038/s41587-020-0501-8

Patel, S., \& Gupta, R. S. (2020). A phylogenomic and comparative genomic framework for resolving the polyphyly of the genus Bacillus: Proposal for six new genera of Bacillus species, Peribacillus gen. nov., Cytobacillus gen. nov., Mesobacillus gen. nov., Neobacillus gen. nov., Metabacillus gen. nov. and Alkalihalobacillus gen. nov. Int J Syst Evol Microbiol, 70(1), 406-438. https://doi.org/10.1099/ijsem.0.003775

Zheng, J., Wittouck, S., Salvetti, E., Franz, C. M. A. P., Harris, H. M. B., Mattarelli, P., O’Toole, P. W., Pot, B., Vandamme, P., Walter, J., Watanabe, K., Wuyts, S., Felis, G. E., Gänzle, M. G., \& Lebeer, S. (2020). A taxonomic note on the genus Lactobacillus: Description of 23 novel genera, emended description of the genus Lactobacillus Beijerinck 1901, and union of Lactobacillaceae and Leuconostocaceae. Int J Syst Evol Microbiol, 70(4), 2782-2858. https://doi.org/10.1099/ijsem.0.004107

PeerJ reviewing PDF | (2020:12:55980:1:1:NEW 13 Jan 2021) 
Figure 1

\section{Analytical Workflow}

* indicates read numbers are detailed post-filtering of diet and host associated reads.

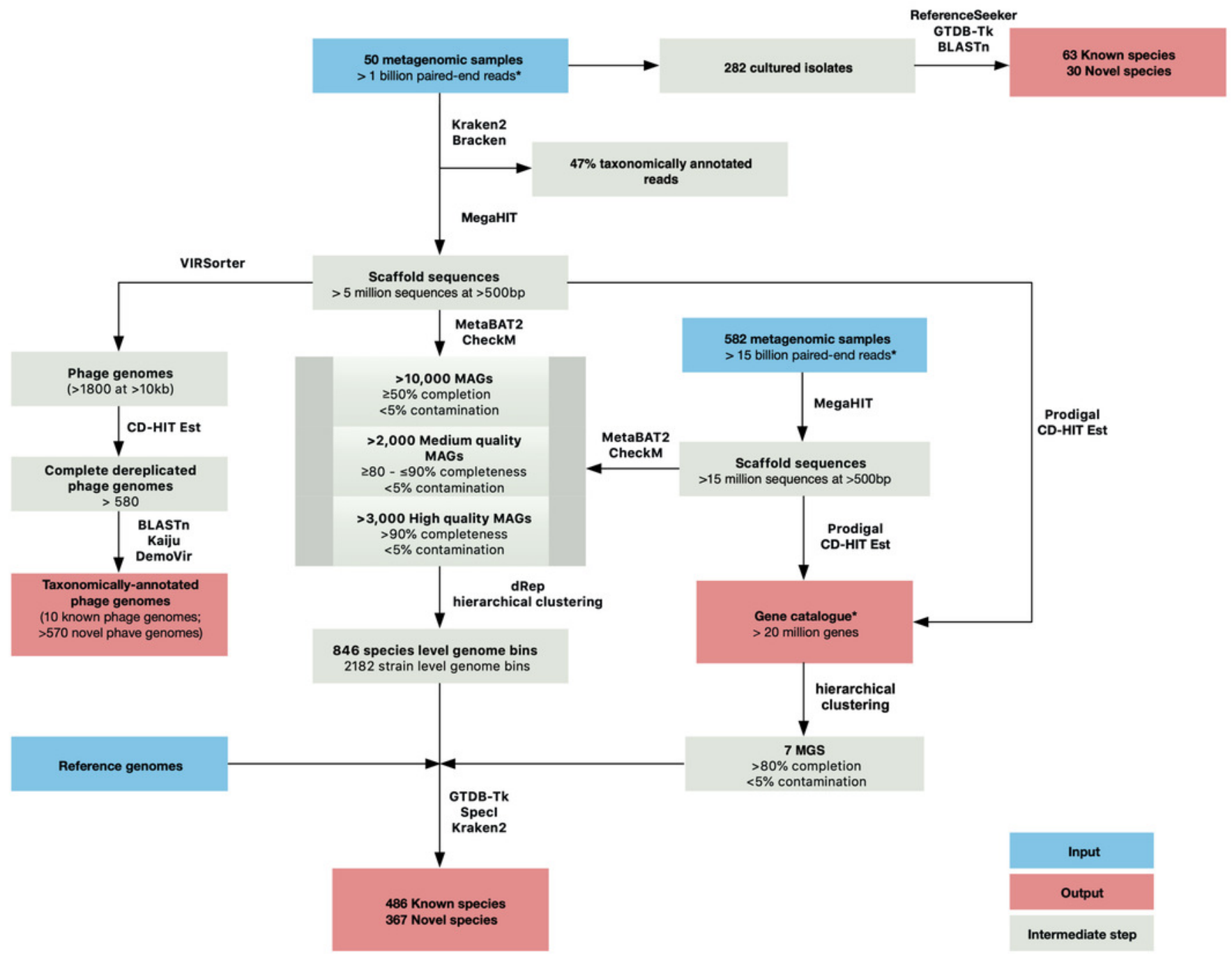


Figure 2

Genome synteny of recovered phage genomes

Synteny plots comparing four novel coliphage genomes recovered from chicken faecal metagenomes (in red) to closest reference genomes. The figure was generated using EasyFig.

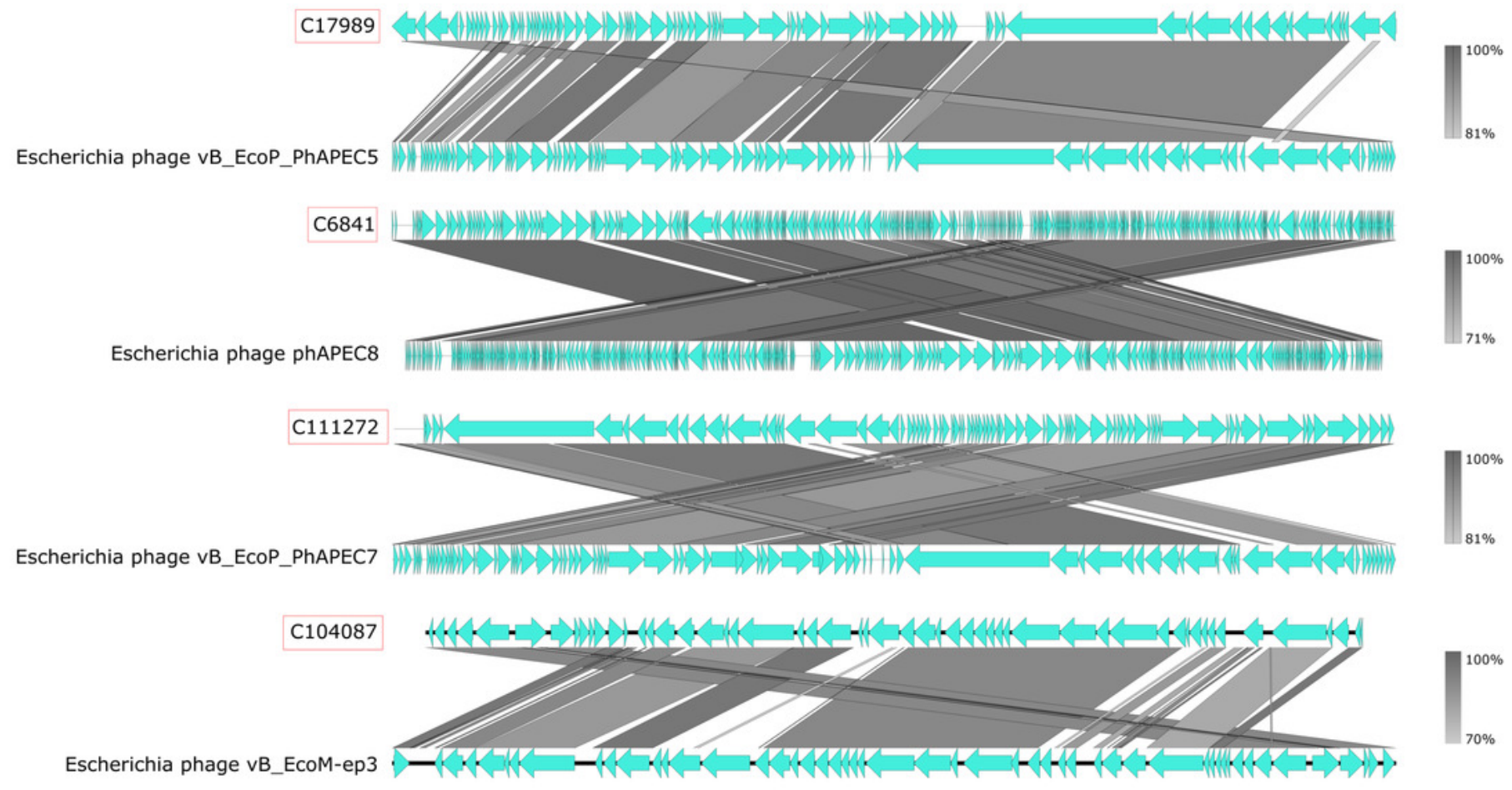




\section{Figure 3}

Coliphage abundance within chicken faecal samples

Coverage of four coliphages and of putative host bacterial species. Only samples in which at least one genome had $\geq 1 \times$ coverage are shown $(n=29)$. All coverage values have been $\log _{10}$ transformed with blue depicting low abundance and red high abundance.

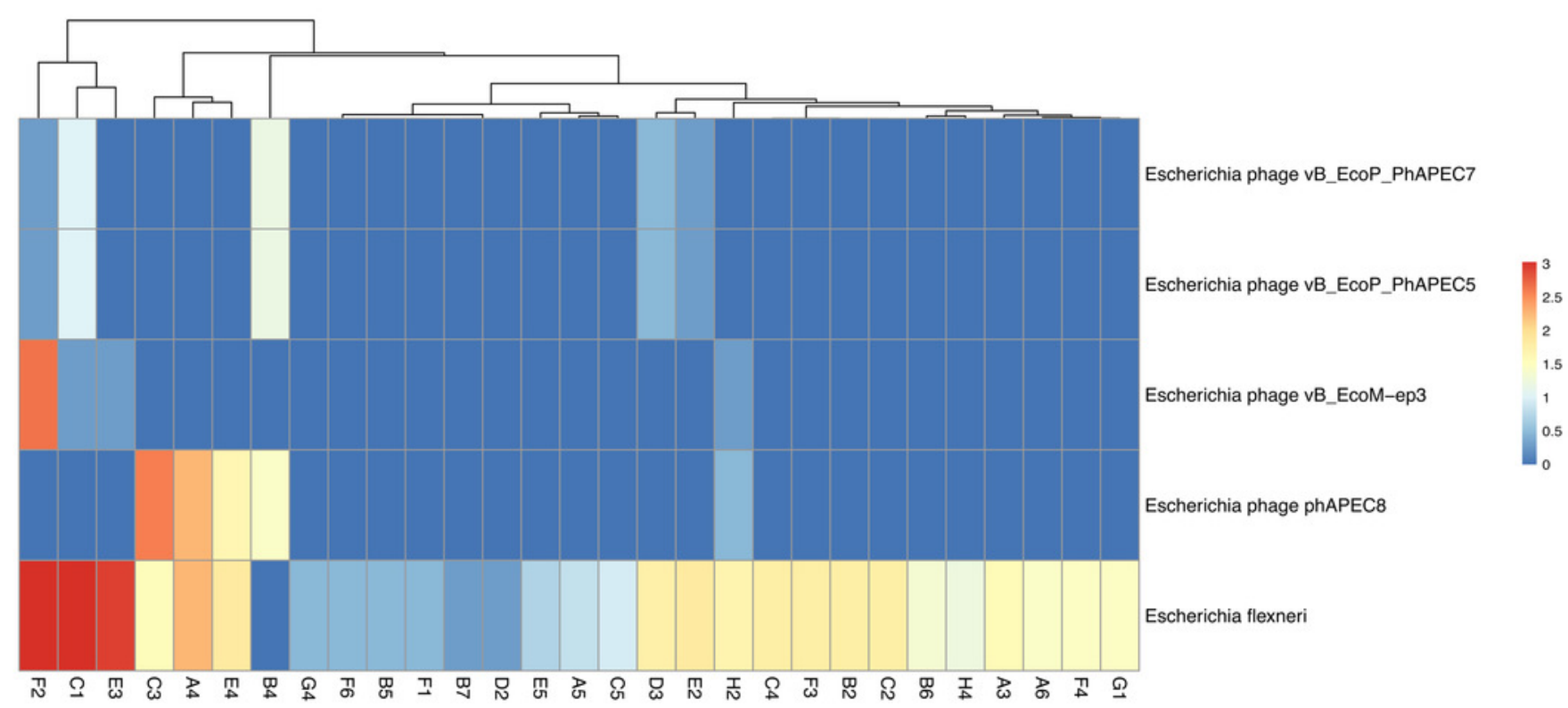




\section{Figure 4}

Phylogenetic tree of draft MGS genomes derived from 820 metagenomic samples of the chicken gut and draft genomes of 93 species cultured from chicken faecal samples

Phylum, generally as assigned by GTDB, is indicated by colour range. Data symbols in the outer layers have been used to describe further characteristics for each draft genomes. Triangles indicate sequence novelty and status of binomial designation within publicly available databases or published research with filled symbols indicating novel species assigned a binomial as part of this research, hollow symbol indicated a known species assigned a binomial as part of this research and no symbol indicated a known species with a well-formed binomial already assigned. Stars are used to indicate isolation source, with filled symbols indicating isolation of species in both culture and metagenomic assembly and hollow symbols indicating isolation in culture alone. Tree branches have been collapsed where duplicate species have been identified by different methodologies. The tree was reconstructed using PhyloPhIAn 3.0.58 against 400 marker genes before reconstruction using FastTree and RAXML of a MAFFT sequence alignment and visualised using the online iTOLV1.4 tool including provision of a heat map according to individual genome length. 


Tree scale: 1
\begin{tabular}{r} 
Genome size \\
\hline 562043 \\
1234728 \\
1907412 \\
2580097 \\
3252781 \\
3925465 \\
4598150 \\
5270834 \\
5943519 \\
6616203 \\
7288888
\end{tabular}
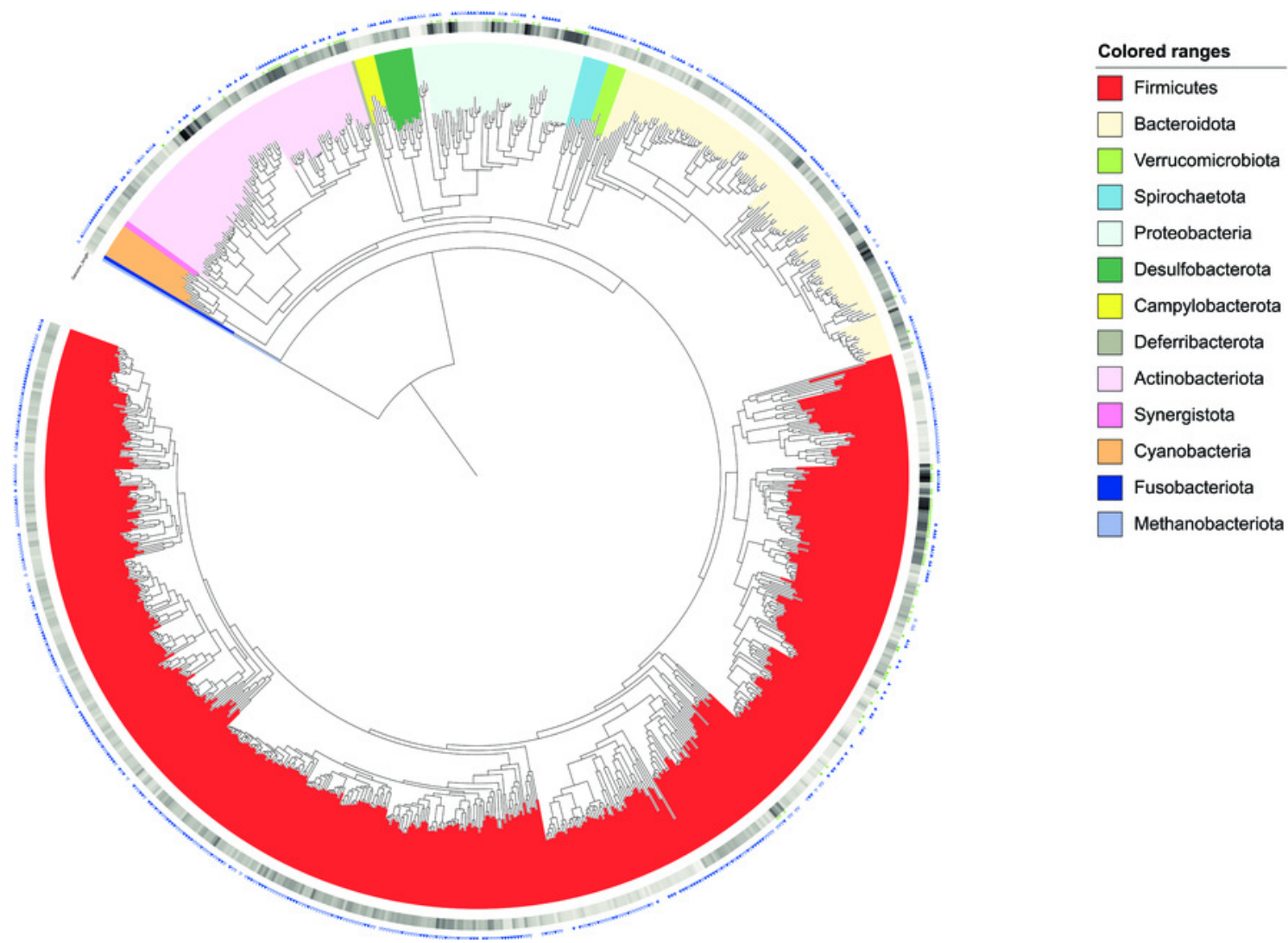


\section{Figure 5}

Phylogenetic tree showing the relationships between Escherichia marmotae, Escherichia whittamii and the other Escherichia species and cryptic clades

The tree was constructed by RAXML maximum likelihood analysis of a core genome alignment generated using Mugsy. The scale bar indicates the number of substitutions per site represented by the branch length shown. Numbers on branches indicate the percentage bootstrap support out of 100 replicates. Strains sequenced as part of this study are highlighted in red. 


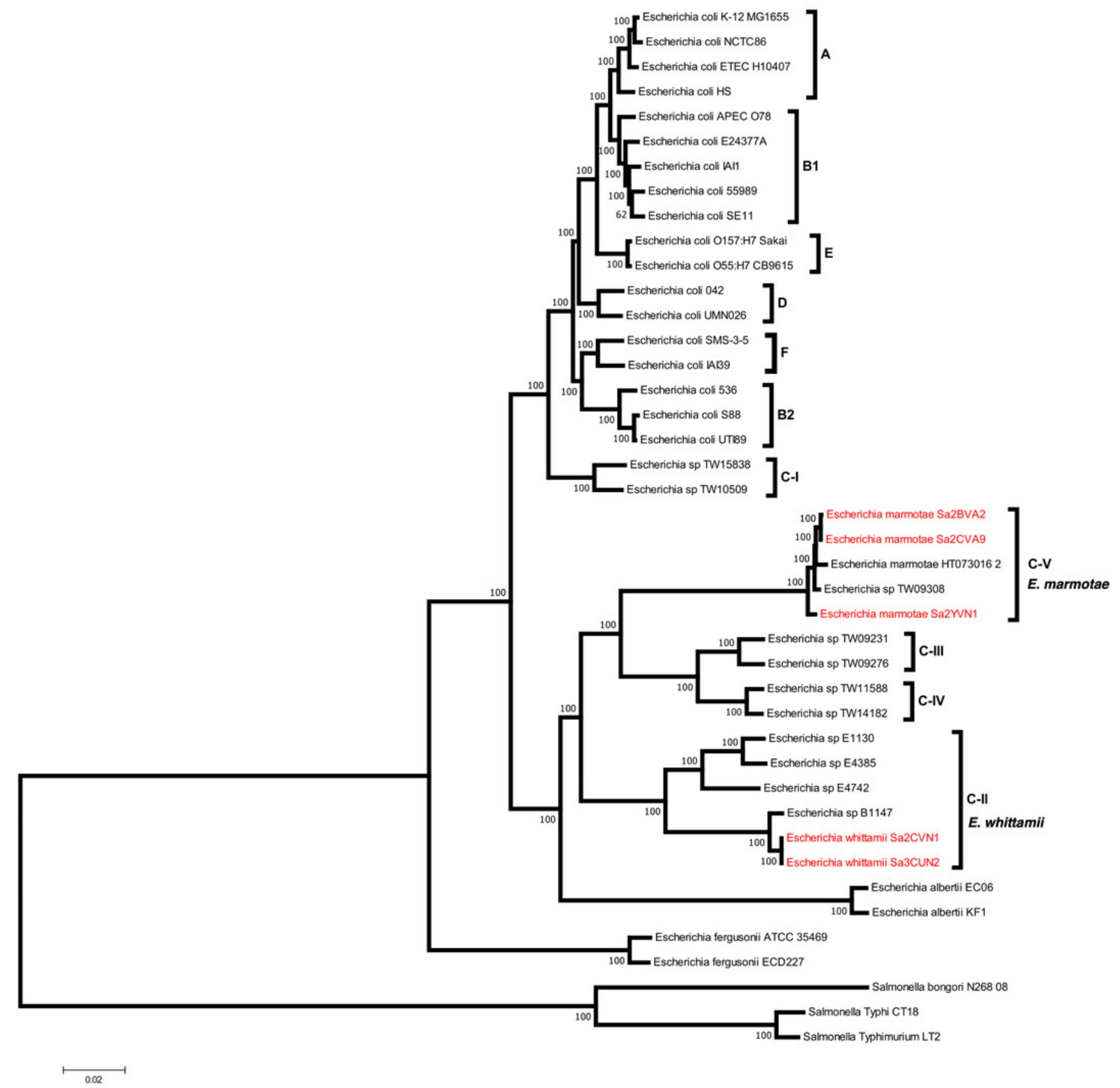




\section{Figure 6}

\section{Sequence novelty}

a. Venn diagram showing shared and unique taxonomic species among three data sources; cultured isolates derived from 6 chicken faecal samples (Cultured species), metagenomic species identified from a combined dataset of $>630$ chicken gastrointestinal metagenome samples (Metagenomic species); MAGs also found by Glendinning et al., 2020 (Glendinning et al., 2020)

b. Percentage of classified metagenomic reads derived from 50 chicken faecal samples according to a standard Kraken 2 database (Previously) and to a standard Kraken 2 database with the addition of the 2344 genomic and metagenomic sequences derived from this study (Now). 
a.

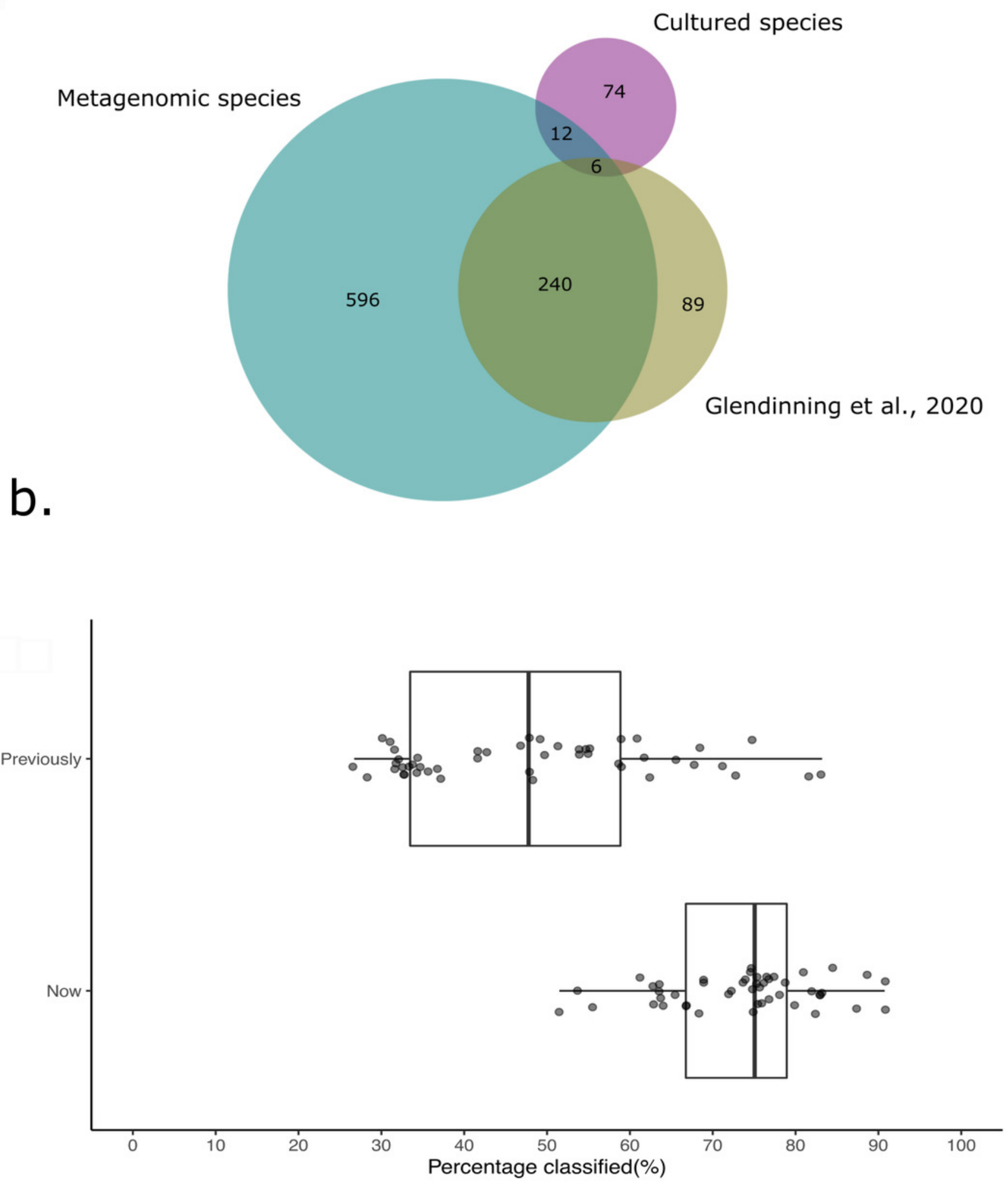




\section{Figure 7}

UpSet plots depicting presence of 820 metagenomic species across all BioProjects included within this study
a. 1x coverage
b. 10x coverage.

Bars are stacked according to taxonomic species novelty, with black-stacked bars depicting novel species and grey depicting species previously described in public databases or published studies. Only intersections with 5 or more species are shown. 


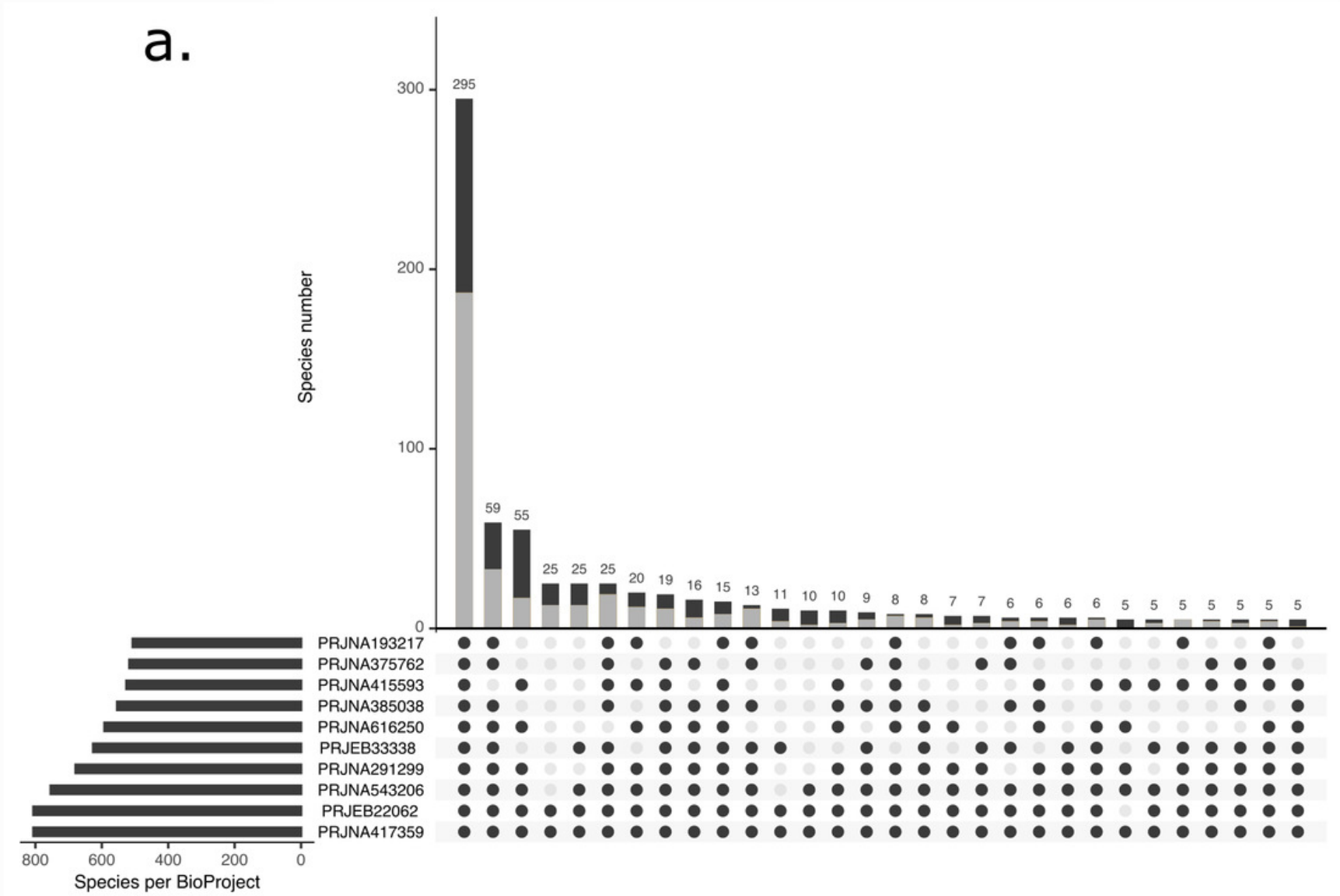

Species per BioProject

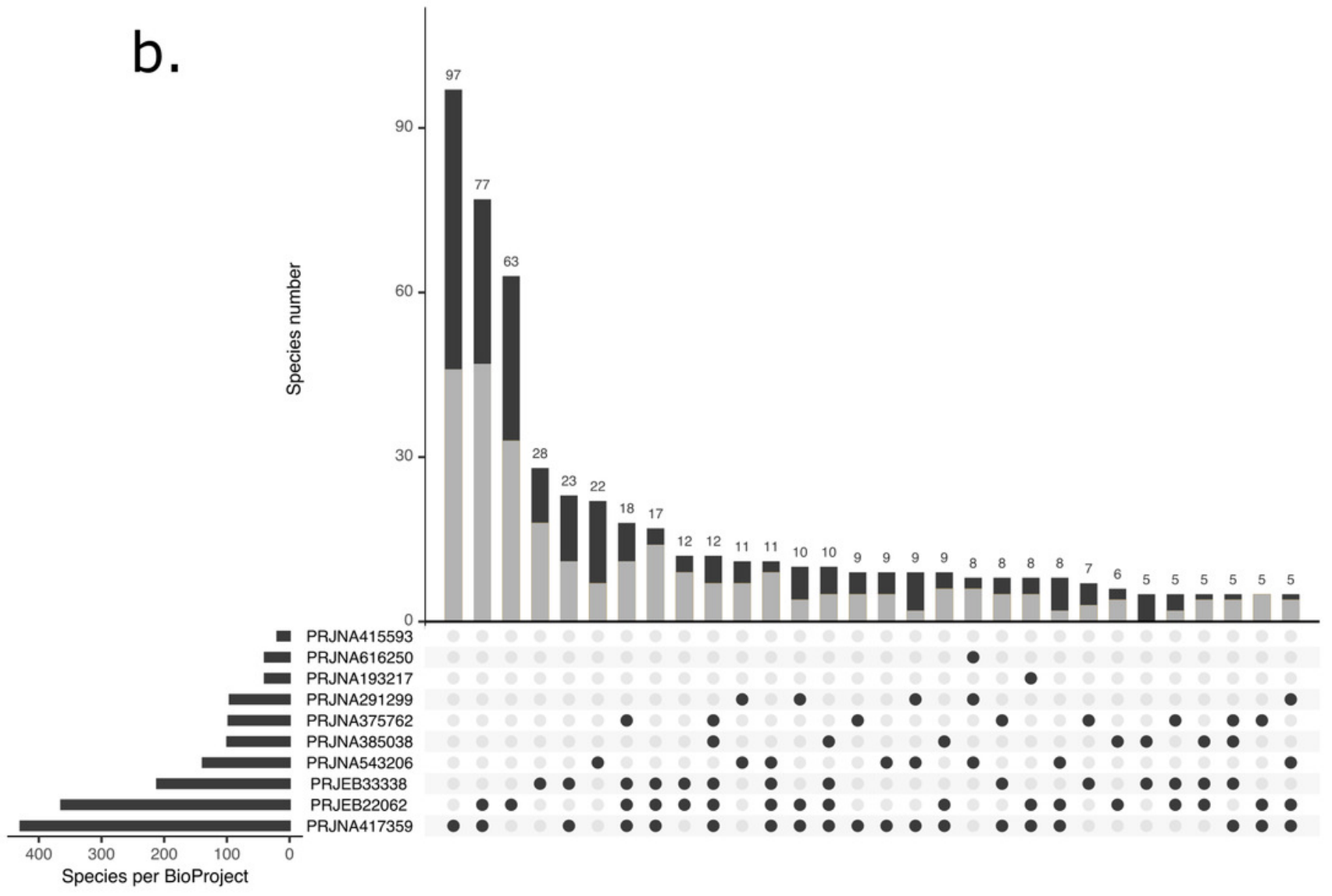

